\title{
IDENTIFIABILITY FOR GRAPHEXES AND THE WEAK KERNEL METRIC
}

\author{
CHRISTIAN BORGS, JENNIFER T. CHAYES, HENRY COHN, \\ AND LÁSZLÓ MIKLÓS LOVÁSZ
}

\begin{abstract}
In two recent papers by Veitch and Roy and by Borgs, Chayes, Cohn, and Holden, a new class of sparse random graph processes based on the concept of graphexes over $\sigma$-finite measure spaces has been introduced. In this paper, we introduce a metric for graphexes that generalizes the cut metric for the graphons of the dense theory of graph convergence. We show that a sequence of graphexes converges in this metric if and only if the sequence of graph processes generated by the graphexes converges in distribution. In the course of the proof, we establish a regularity lemma and determine which sets of graphexes are precompact under our metric. Finally, we establish an identifiability theorem, characterizing when two graphexes are equivalent in the sense that they lead to the same process of random graphs.
\end{abstract}

\section{Contents}

1. Introduction

2. Definitions and statements of main results 3

3. Preliminaries 19

4. Tightness 43

5. Regularity lemma and compactness 50

6. Subgraph counts $\quad 59$

6.1. Proof of the counting lemma 60

6.2. GP-convergence and subgraph counts $\quad 65$

6.3. Metric convergence implies GP-convergence 71

7. Sampling 71

7.1. Closeness of graphexes implies closeness of samples $\quad 72$

7.2. Samples converge to graphex 76

7.3. Proofs of Theorem 2.18, Proposition 2.13, and Theorem $2.23 \quad 80$

8. Identifiability 80

8.1. Infimum is minimum 81

8.2. Canonical graphex 86

9. Uniform integrability and uniform tail regularity 93

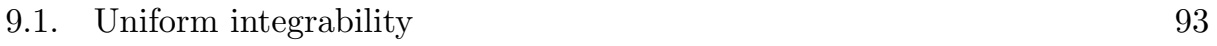

9.2. Uniform tail regularity 96

$\begin{array}{ll}\text { Acknowledgements } & 101\end{array}$

$\begin{array}{lll}\text { Appendix A. Local finiteness } & 102\end{array}$

$\begin{array}{ll}\text { Appendix B. Sampling with loops } & 106\end{array}$

$\begin{array}{ll}\text { References } & 108\end{array}$ 


\section{INTRODUCTION}

The theory of graph limits has been extensively developed for dense graph sequences $[7,21,22,8,10,9]$, but the sparse case is not as well understood. In this paper, we study a model introduced and studied in a sequence of papers $[11,26,3,17,25,18,4]$ based on the notion of graphexes. In contrast to the graphons of the dense theory, which are symmetric two-variable functions defined over a probability space, graphexes are defined over $\sigma$-finite measure spaces, and, in addition to a graphon part $W$, contain two other components: a function $S$ taking values in $\mathbb{R}_{+}$, and a parameter $I \in \mathbb{R}_{+}$. Formally, the graphex is then the quadruple $\mathbb{W}=(W, S, I, \boldsymbol{\Omega})$, where $\boldsymbol{\Omega}=(\Omega, \mathcal{F}, \mu)$ is the underlying measure space.

A graphex then leads to a process $\left(G_{T}(\mathbb{W})\right)_{T \geq 0}$ of random graphs as follows: starting from a Poisson process over $\Omega$ with intensity $T \mu$, one attaches Poisson $\left(T S\left(x_{i}\right)\right)$ leaves to each Poisson point $x_{i}$, and in addition, joins two Poisson points $x_{i}, x_{j}$ with probability $W\left(x_{i}, x_{j}\right)$. Finally, one adds Poisson $\left(T^{2} I\right)$ isolated edges not connected to any of the other points. Removing isolated vertices as well as the labels of the remaining vertices gives a graphex process $\left(G_{T}(\mathbb{W})\right)_{T \geq 0}$ of unlabeled graphs sampled from $\mathbb{W}$.

Several notions of convergence for graphexes were introduced in [3] and [25] and further studied in [18]. Among these notions, we will be particularly interested in graphex process convergence (GP-convergence), which was introduced in [25]. A sequence of graphexes is GP-convergent if the random graph processes generated by the graphexes in the sequence converge. It was pointed out in [18] that GPconvergence can be metricized using the abstract theory of probability measures over Polish spaces, but this does not give a very explicit metric on graphexes; in fact, it does not even allow us to determine when two graphexes lead to the same random graph process.

In this paper, we introduce a concrete notion of distance for graphexes that is equivalent to GP-convergence, which can be thought of as corresponding to the "cut distance" in the dense case. For reasons we explain in the next section, we call it the "weak kernel distance". We show that convergence in this distance is equivalent to GP-convergence.

In general, the set of all graphexes is not compact. Indeed, it is not difficult to show that for a set to be compact under GP-convergence, certain uniform boundedness assumptions are necessary on the set of graphexes, which we call "tightness". As a part of our proof that our weak kernel distance metricizes GP-convergence, we develop a (Frieze-Kannan-type) regularity lemma for graphexes and show that the sets that are precompact under the weak kernel metric are precisely those that are tight.

Finally, we prove an identifiability theorem, showing to what extent a graphex can be identified from its graphex process. Formulated differently, we give a characterization of the equivalence classes of graphexes, where two graphexes are called equivalent if they give rise to the same graphex process. Generalizing a construction that was developed by Janson for the dense case [16], we assign to each graphex $\mathbb{W}$ a "canonical version" $\widehat{\mathbb{W}}$ such that $\mathbb{W}$ is a pullback of $\widehat{\mathbb{W}}$ and show that if two graphexes are equivalent, then their canonical versions are isomorphic up to measure zero changes. This in turn will imply that two graphexes $\mathbb{W}_{1}$ and $\mathbb{W}_{2}$ are equivalent if and only if there is a third graphex $\mathbb{W}_{3}$ (which can be taken to be their canonical graphex) such that after restricting the two graphexes to their "support" 
(strictly speaking, we have to restrict them to their "degree support", a notion we will define in the next section) both $\mathbb{W}_{1}$ and $\mathbb{W}_{2}$ are pullbacks of $\mathbb{W}_{3}$. We note that this proves a conjecture of Janson; see Remark 5.4 in [17].

We note that in this paper we treat graphexes slightly differently from the definition in $[26,17,25,18,4]$. Namely, as in [3], we follow the convention from the theory of dense graph limits, and define the graphex process corresponding to a graphex as a process of graphs without loops. Indeed, we believe that a theory with loops is most naturally embedded into a more general theory of graphex processes with multi-edges and loops, which is beyond the scope of this paper.

Nonetheless, it is worth pointing out that the reader interested in the theory with loops (but not multi-edges) can derive many results for this theory from those developed here, even though some of the theorems will need to be modified to accommodate additional technical complications. For the identifiability theorem, this is done in Appendix B.

Finally, we note that while signed graphexes (i.e., graphexes for which $W, S$ and $I$ are not necessarily non-negative) do not make much sense if we want to use them to generate a random graph process, they are quite natural from an analytic point of view. Indeed, we will prove several of our results for signed graphexes. Still, the goal of this paper is to study unsigned graphexes, and our results on signed graphexes should be considered more of an aside at this point.

\section{Definitions and Statements of main Results}

Definition 2.1. A graphex $\mathbb{W}=(W, S, I, \boldsymbol{\Omega})$ consists of a $\sigma$-finite measure space $\boldsymbol{\Omega}=(\Omega, \mathcal{F}, \mu)$, a symmetric measurable function $W: \Omega \times \Omega \rightarrow[0,1]$, a measurable function $S: \Omega \rightarrow \mathbb{R}^{+}$, and a nonnegative real number $I$ such that the following local finiteness conditions hold:

(1) $W(\cdot, x)$ is integrable for almost all $x \in \Omega$, and

(2) there exists a measurable subset $\Omega^{\prime} \subseteq \Omega$ such that $\mu\left(\Omega \backslash \Omega^{\prime}\right)<\infty$ and $\left.\mathbb{W}\right|_{\Omega^{\prime}}$ is integrable.

The quadruple will be called a signed graphex if instead of taking values in $[0,1]$ and $\mathbb{R}_{+}, W, S$ and $I$ take values in $\mathbb{R}$. The graphex $\mathbb{W}=(W, S, I, \boldsymbol{\Omega})$ is called integrable if

$$
\|\mathbb{W}\|_{1}:=\int_{\Omega \times \Omega}|W(x, y)| d \mu(x) d \mu(y)+2 \int_{\Omega}|S(x)| d \mu(x)+2|I|<\infty,
$$

and the restriction $\left.\mathbb{W}\right|_{\Omega^{\prime}}$ of $\mathbb{W}$ to $\Omega^{\prime} \subseteq \Omega$ is defined as the quadruple $\mathbb{W}^{\prime}=$ $\left(W^{\prime}, S^{\prime}, I^{\prime}, \boldsymbol{\Omega}^{\prime}\right)$ with $\boldsymbol{\Omega}^{\prime}=\left(\Omega^{\prime}, \mathcal{F}^{\prime}, \mu^{\prime}\right)$, where $\mathcal{F}^{\prime}=\left\{A \in \mathcal{F}: A \subseteq \Omega^{\prime}\right\}, \mu^{\prime}$ is the restriction of $\mu$ to $\mathcal{F}^{\prime}, S^{\prime}$ is the restriction of $S$ to $\Omega^{\prime}$, and $W^{\prime}$ is the restriction of $W$ to $\Omega^{\prime} \times \Omega^{\prime}$.

We often refer to $\mathbb{W}$ as a signed graphex over $\boldsymbol{\Omega}$, and we will refer to the function $W$ as a graphon, or the graphon part of $\mathbb{W}$. Similarly, $S$ will be called a star intensity, or the star part of $\mathbb{W}$, and $I$ will be called a dust density, or the dust part of $\mathbb{W}$. (The reason for this terminology will become clear when we discuss the random graph process generated by an unsigned graphex $\mathbb{W}$; as we will see, the star part of $\mathbb{W}$ will lead to stars, and the dust part will lead to isolated edges, which we call dust following [18].) If two signed graphexes $\mathbb{W}_{1}, \mathbb{W}_{2}$ are defined on the same space $\boldsymbol{\Omega}$, then we say that $\mathbb{W}_{1}=\mathbb{W}_{2}$ almost everywhere if $W_{1}=W_{2}$ almost everywhere, $S_{1}=S_{2}$ almost everywhere, and $I_{1}=I_{2}$. 
We define the marginal of a graphex $\mathbb{W}=(W, S, I, \boldsymbol{\Omega})$ over $\boldsymbol{\Omega}=(\Omega, \mathcal{F}, \mu)$ as the a.e. finite function $D_{\mathbb{W}}: \Omega \rightarrow \mathbb{R}_{+}$defined by

$$
D_{\mathbb{W}}(x)=D_{W}(x)+S(x) \quad \text { where } \quad D_{W}(x)=\int_{\Omega} W(x, y) d \mu(y) .
$$

We say that $\mathbb{W}$ has $D$-bounded marginals if $\left\|D_{\mathbb{W}}\right\|_{\infty} \leq D$. Finally, we define its degree support as the set

$$
\operatorname{dsupp} \mathbb{W}=\left\{x \in \Omega: D_{\mathbb{W}}(x)>0\right\} .
$$

Note that $\mathbb{W}$ is integrable if and only if its marginals are integrable.

Given a graphex $\mathbb{W}$, we will define a stochastic process $\left(\mathcal{G}_{T}(\mathbb{W})\right)_{T \geq 0}$ indexed by $T \in \mathbb{R}_{+}$and taking values in the set of graphs with labels in $\mathbb{R}_{+}$. To make this precise, we need to define a $\sigma$-algebra over the set of countable graphs with vertices in $\mathbb{R}_{+}$. To this end, we first define the adjacency measure $\xi_{G}$ of a countable graph $G$ with vertices in $\mathbb{R}_{+}$as the measure $\xi_{G}$ on $\mathbb{R}_{+}^{2}$ given by

$$
\xi_{G}=\sum_{t, t^{\prime} \in V(G):\left\{t, t^{\prime}\right\} \in E(G)} \delta_{\left(t, t^{\prime}\right)} .
$$

We call $\xi$ an adjacency measure if there exists a countable graph $G$ such that $\xi=\xi_{G}$. We then equip the set of adjacency measures with the smallest $\sigma$-algebra such that the maps $\xi \mapsto \xi(A)$ are measurable for all bounded Borel sets $A \subseteq \mathbb{R}_{+}^{2}$, and the set of countable graphs with vertices in $\mathbb{R}_{+}$with the smallest $\sigma$-algebra such that the maps $G \mapsto \xi_{G}$ are measurable.

A graphex $\mathbb{W}=(W, S, I, \boldsymbol{\Omega})$ then generates a family $\left(\mathcal{G}_{T}(\mathbb{W})\right)_{T \geq 0}$ of random graphs as follows: we start with a Poisson point process with intensity $\lambda \times \mu$ on $\mathbb{R}_{+} \times \Omega$, where $\lambda$ is the Lebesgue measure on $\mathbb{R}_{+}$, and then connect two points $(t, x)$ and $\left(t^{\prime}, x^{\prime}\right)$ of the Poisson process with probability $W\left(x, x^{\prime}\right)$, independently for all pairs of points. For each point of the Poisson process $(t, x)$, we take another Poisson point process on $\mathbb{R}_{+}$with intensity $S(x) \lambda$, and connect $(t, x)$ to a vertex with "birth time" $t_{i}$ for each point $t_{i}$ in the process. We also take a Poisson process with intensity $I(\lambda \times \lambda)$ on $\mathbb{R}_{+}^{2}$, and for each point $\left(t_{x}, t_{y}\right)$ we take an isolated edge between vertices with birth time $t_{x}$ and $t_{y}$. If we ignore the labels in the feature space $\Omega$ and delete the vertices with degree zero, this leads to an infinite graph $\mathcal{G}_{\infty}(\mathbb{W})$ with vertices labeled by their birth time $t \in \mathbb{R}_{+}$. We then define $\mathcal{G}_{T}(\mathbb{W})$ by first taking the induced subgraph on the set of vertices which lie in $[0, T]$ and then deleting vertices whose neighbors in $\mathcal{G}_{\infty}(\mathbb{W})$ all lie outside the interval $[0, T]$.

We will refer to the part of $\mathcal{G}_{\infty}(\mathbb{W})$ generated with the help of the dust intensity $I$ as the dust part of $\mathcal{G}_{\infty}(\mathbb{W})$, and as the part generated with the help of the star intensity $S$ as the stars in $\mathcal{G}_{\infty}(\mathbb{W})$. While it may not be a priori clear whether these parts can be inferred from just observing the infinite graph $\mathcal{G}_{\infty}(\mathbb{W})$, this is actually the case, a fact which was first noted in Remark 5.4 in [17]: almost surely, the dust part consists of all edges in $\mathcal{G}_{\infty}(\mathbb{W})$ that are isolated, the star part consists of all edges with one vertex of degree one and a second vertex of infinite degree, and the remaining edges are generated by the graphon part of $\mathbb{W}$ and have two endpoints with infinite degree.

Definition 2.2. Let $\mathbb{W}$ be a graphex, let $\left(\mathcal{G}_{T}(\mathbb{W})\right)_{T \geq 0}$ be the random family of graphs defined above, and let $\xi[\mathbb{W}]$ be the random adjacency measure $\xi_{\mathcal{G}_{\infty}(\mathbb{W})}$. We call the stochastic process $\left(\mathcal{G}_{T}(\mathbb{W})\right)_{T \geq 0}$ the graphex process generated by $\mathbb{W}$, and the adjacency measure $\xi[\mathbb{W}]$ the adjacency measure generated by $\mathbb{W}$. We say two 
graphexes are equivalent, if the graphex processes generated by these graphons are equal in law.

Remark 2.3. (1) Following [4], we defined a graphex process as a stochastic process taking values in a space of graphs with labels in $\mathbb{R}_{+}$. Alternatively, one might want to define a graphex process as a process taking values in the space of unlabeled graphs without isolated vertices. In our current context, this would correspond to ignoring the time labels of the graphs in $\mathcal{G}_{T}(\mathbb{W})$, leading to a graph which we denote by $G_{T}(\mathbb{W})$. When it is important to distinguish them, we will refer to the process $\left(G_{T}(\mathbb{W})\right)_{T \geq 0}$ as the unlabeled graphex process corresponding to $\mathbb{W}$, and to the process $\left(\mathcal{G}_{T}(\mathbb{W})\right)_{T \geq 0}$ as the labeled graphex process corresponding to $\mathbb{W}$. Note that it is easy to recover $\mathcal{G}_{T}(\mathbb{W})$ from $G_{T}(\mathbb{W})$ : just assign i.i.d. labels chosen uniformly at random in $[0, T]$ to all vertices. A related observation is the fact that $G_{T}(\mathbb{W})$ can be generated by first choosing $\left(x_{i}\right)_{i \geq 1}$ according to a Poisson process with intensity $T \mu$ in $\Omega$, then connecting $i$ and $j$ with probability $W\left(x_{i}, x_{j}\right)$, then adding a star whose number of leaves are chosen as a Poisson random variable with mean $T S\left(x_{i}\right)$ to each point of the process $\left(x_{i}\right)_{i \geq 1}$, and finally adding independent edges with rate $I T^{2}$. Forgetting the labels then gives us $G_{T}(\mathbb{W})$. Relabeling each vertex in the resulting graph independently by a uniform $t \in[0, T]$, we obtain $\mathcal{G}_{T}(\mathbb{W})$.

(2) It is sometimes convenient to assign a feature value to the endpoints of the isolated edges generated from the dust part $I$ in the graphex, as well as to the leaves of the stars generated using the function $S$. For our purpose, we will say that these vertices have the feature label $\infty$, and we will extend the marginal $D_{\mathbb{W}}$ to $\Omega \cup\{\infty\}$ by setting

$$
D_{\mathbb{W}}(\infty)=\int_{\Omega} S(x) d \mu(x)+2 I .
$$

Note that with this notation, $\|\mathbb{W}\|_{1}=\int_{\Omega} D_{\mathbb{W}}(x) d \mu(x)+D_{\mathbb{W}}(\infty)$.

(3) In view of (2), one might want to equip the extended feature space $\widetilde{\Omega}=\Omega \cup\{\infty\}$ with a $\sigma$-finite measure by keeping the original measure on $\Omega$, and assigning some finite measure $Q=\widetilde{\mu}(\infty)$ to the feature value $\infty$, giving a new $\sigma$-finite measure space $\widetilde{\boldsymbol{\Omega}}_{Q}$. On $\widetilde{\boldsymbol{\Omega}}_{Q}$, one can then define a graphex of the form $\widetilde{\mathbb{W}}_{Q}=\left(\widetilde{W}_{Q}, 0,0, \widetilde{\boldsymbol{\Omega}}\right)$ by setting $\widetilde{W}_{Q}$ equal to $W$ on $\Omega \times \Omega$ and to $2 I / Q^{2}$ on $\{\infty\} \times\{\infty\}$, and by setting $\widetilde{W}(x, \infty)=S(x) / Q$ and $\widetilde{W}(\infty, y)=S(y) / Q$ if only one of the two features $x, y$ lies in $\Omega$. With this construction, $D_{\widetilde{W}_{Q}}(\infty)=D_{\mathbb{W}}(\infty) / Q, D_{\widetilde{W}_{Q}}(x)=D_{\mathbb{W}}(x)$ if $x \in \Omega$, and $\|\mathbb{W}\|_{1}=\left\|\widetilde{W}_{Q}\right\|_{1}=\int d \mu(x) D_{\widetilde{W}_{1}}+Q D_{\widetilde{W}}(\infty)$, with the notation in (2) corresponding to the case $Q=1$. It is clear that the graphon process generated from $\widetilde{\mathbb{W}}$ cannot have exactly the same distribution as the one generated from $\mathbb{W}$ unless $I$ and $S$ are zero (to see this, note that in $G_{\infty}(\widetilde{\mathbb{W}})$, all vertices have infinite degrees, while $G_{\infty}(\mathbb{W})$ has vertices of degree one). But one might wonder whether the process generated from the "pure graphon" $\widetilde{\mathbb{W}}$ approximates the one generated from $\mathbb{W}$. As we will see in Remark 7.7, this is indeed the case, in the sense that for any fixed $T$, the distribution of $G_{T}\left(\widetilde{\mathbb{W}}_{Q}\right)$ converges to $G_{T}(\mathbb{W})$.

It is relatively easy to see that the local finiteness conditions (1) and (2) from Definition 2.1 imply that the adjacency measure $\xi[\mathbb{W}]$ is a.s. locally finite (i.e., $\xi[\mathbb{W}](A)<\infty$ for all bounded Borel sets $\left.A \subset \mathbb{R}_{+}^{2}\right)$, or equivalently, that for all $T<\infty$, the graphs $\mathcal{G}_{T}(\mathbb{W})$ are a.s. finite. It turns out that these conditions are also necessary for the local finiteness of $\xi[\mathbb{W}]$. This is the main statement of the 
following proposition, which we will prove in Appendix A. For graphexes over $\mathbb{R}_{+}$ equipped with the Lebesgue measure, a similar condition was established in [26], building on the work of [19] (the condition considered by [26] and [19] is the same as our condition (E) below, specialized to the case $D=1$, even though it is clear that both [26] and [19] knew that for graphexes over $\mathbb{R}_{+}$, conditions (D) and (E) are equivalent.) To state the proposition, we use the notation $\left\{D_{\mathbb{W}}>D\right\}$ for the set $\left\{x \in \Omega: D_{\mathbb{W}}(x)>D\right\}$, while $\left\{D_{W}>D\right\},\left\{D_{\mathbb{W}} \leq D\right\}$, and $\left\{D_{W} \leq D\right\}$ are defined analogously.

Proposition 2.4. Let $\mathbb{W}=(W, S, I, \boldsymbol{\Omega})$ be a 4-tuple consisting of a $\sigma$-finite measure space $\boldsymbol{\Omega}=(\Omega, \mathcal{F}, \mu)$, a symmetric measurable function $W: \Omega \times \Omega \rightarrow[0,1]$, a measurable function $S: \Omega \rightarrow \mathbb{R}^{+}$, and a nonnegative real number $I$. Then the local finite conditions (1) and (2) from Definition 2.1 are equivalent to the local finiteness of the adjacency measure generated by $\mathbb{W}$. If we assume condition (1), then following are equivalent:

(A) The graphex $\mathbb{W}$ obeys the local finiteness condition (2).

(B) For all $D>0, \mu\left(\left\{D_{\mathbb{W}}>D\right\}\right)<\infty$ and $\left.\mathbb{W}\right|_{\left\{D_{\mathbb{W}} \leq D\right\}}$ is integrable.

(C) There exists a $D>0$ such that $\mu\left(\left\{D_{\mathbb{W}}>D\right\}\right)<\infty$ and $\left.\mathbb{W}\right|_{\left\{D_{\mathbb{W}} \leq D\right\}}$ is integrable.

(D) For all $D>0, \mu\left(\left\{D_{W}>D\right\}\right)<\infty$, and both $\left.W\right|_{\left\{D_{W} \leq D\right\}}$ and $\min \{S, 1\}$ are integrable.

(E) There exists a $D>0$ such that $\mu\left(\left\{D_{W}>D\right\}\right)<\infty$, and both $\left.W\right|_{\left\{D_{W} \leq D\right\}}$ and $\min \{S, 1\}$ are integrable.

Note that this proposition implies in particular that a graphex with bounded marginals is integrable, since for graphexes with $\left\|D_{\mathbb{W}}\right\|_{\infty} \leq D$ the graphex $\mathbb{W}$ and the graphex $\left.\mathbb{W}\right|_{\left\{D_{\mathbb{W}}\right\} \leq D}$ are the same.

Having defined the graphex process associated with a graphex $\mathbb{W}$, there are several natural questions one might want to answer. In particular, one might want to characterize when two graphexes lead to the same process, i.e., when $\xi[\mathbb{W}]$ and $\xi\left[\mathbb{W}^{\prime}\right]$ have the same distribution. More generally, one might want to define a metric on the set of graphexes such that the distributions of $\xi[\mathbb{W}]$ and $\xi\left[\mathbb{W}^{\prime}\right]$ are close if $\mathbb{W}$ and $\mathbb{W}^{\prime}$ are close. Addressing these questions is one of the main goals of this paper.

Before discussing this further, it will be useful to embed the theory of graphex processes into the general theory of locally finite point processes. To this end, we first introduce the set $\mathcal{N}=\mathcal{N}\left(\mathbb{R}_{+}^{2}\right)$ of locally finite counting measures on $\mathbb{R}_{+}^{2}$ (i.e., the set of measures $\xi$ such that $\xi(A)$ is a finite, non-negative integer for all bounded Borel sets $A \subset \mathbb{R}_{+}^{2}$ ), and equip it with the Borel $\sigma$-algebra inherited from the vague topology (defined as the coarsest topology for which the maps $\xi \mapsto \int f d \xi$ are continuous for all continuous functions $f: \mathbb{R}_{+}^{2} \rightarrow \mathbb{R}_{+}$with compact support). As shown in, e.g., [13], Appendix A2.6, the vague topology on $\mathcal{N}$ can be metricized in such a way that $\mathcal{N}$ becomes a complete, separable metric space, making $\mathcal{N}$ into a Polish space, and the Borel $\sigma$-algebra inherited from this topology is the smallest $\sigma$-algebra such that for all bounded Borel sets $A \subset \mathbb{R}_{+}^{2}$ the maps $\mu \mapsto \mu(A)$ are measurable.

As usual, a locally finite point process on $\mathbb{R}_{+}^{2}$ is then defined as a random measure on $\mathcal{N}\left(\mathbb{R}_{+}^{2}\right)$ equipped with this Borel algebra, and convergence in distribution is defined as weak convergence in the set of probability measures on $\mathcal{N}$, so that convergence in distribution of a sequence of locally finite point process $\xi_{n}$ on $\mathbb{R}_{+}^{2}$ to a locally finite point process $\xi$ is defined by the condition that $\mathbb{E}\left[F\left(\xi_{n}\right)\right] \rightarrow \mathbb{E}[F(\xi)]$ 
for all continuous, bounded functions $F$, with continuity defined with respect to the vague topology on $\mathcal{N}$. As observed in [18], the fact that $\mathcal{N}$ is Polish makes the set of probability distributions on $\mathcal{N}$ a Polish space as well (see, e.g., [1], Appendix III for a proof), showing that convergence in distribution for locally finite point processes on $\mathbb{R}_{+}^{2}$ can be metricized.

Next we consider the set $\hat{\mathfrak{G}}$ of simple graphs $G$ with vertices in $\mathbb{R}_{+}$such that (a) no vertex in $G$ is isolated, and (b) for all $T<\infty$, the induced subgraph of $G$ on $V(G) \cap[0, T]$ is finite. We also consider the subset $\hat{\mathfrak{G}}_{0}$ of finite graphs in $\hat{\mathfrak{G}}$. The map $G \mapsto \xi(G)$ then gives a one-to-one map between graphs in $\hat{\mathfrak{G}}$ and adjacency measures. In particular, $\hat{\mathfrak{G}}$ and its subset $\hat{\mathfrak{G}}_{0}$ inherit the vague topology and corresponding Borel $\sigma$-algebra from $\mathcal{N}$. In this language, the graphex process $\left(\mathcal{G}_{T}(\mathbb{W})\right)_{T \geq 0}$ then becomes a CADLAG stochastic process with values in $\hat{\mathfrak{G}}_{0}$ indexed by a time $T \in \mathbb{R}_{+}$.

Note that $\hat{\mathfrak{G}}$ should be distinguished from the set of unlabeled countable graphs without isolated vertices, $\mathfrak{G}$. While we will not equip $\mathfrak{G}$ with any topology, the set of finite unlabeled graphs without isolated vertices, denoted by $\mathfrak{G}_{0}$, will be given the discrete topology. In this language, the unlabeled graphex process $\left(G_{T}(\mathbb{W})\right)_{T \geq 0}$ introduced in Remark 2.3 is then a CADLAG process with values in $\mathfrak{G}_{0}$.

In [18], various notions of convergence for graphons and graphexes (proposed originally in [3] and [25]) were studied. Here we are most interested in what [25] introduces as GP-convergence, where GP stands for graphex process. This notion is closely related to the notion of sampling convergence for graphs introduced in [4]; see Lemma 5.4 in that paper, as well as the discussion at the end of this section. Janson showed that the following are equivalent if $\mathbb{W}, \mathbb{W}_{1}, \mathbb{W}_{2}, \ldots$ are graphexes:

(1) $\xi\left(G\left(\mathbb{W}_{n}\right)\right) \rightarrow \xi(G(\mathbb{W}))$ in distribution.

(2) For every $T<\infty, \xi\left(\mathcal{G}_{T}\left(\mathbb{W}_{n}\right)\right) \rightarrow \xi\left(\mathcal{G}_{T}\left(\mathbb{W}_{n}\right)\right)$ in distribution.

(3) For every $T<\infty, G_{T}\left(\mathbb{W}_{n}\right) \rightarrow G_{T}(W)$ in distribution.

Following [25] we call this notion of convergence GP-convergence, and say that $\mathbb{W}_{n}$ is GP-convergent to $\mathbb{W}$ if one of these equivalent conditions holds.

As already alluded to above, Janson also observed that by the abstract theory of probability measures over Polish spaces, this notion of convergence can be metricized, turning the set of graphexes into a complete, separable metric space. But this abstract theory does not give a very explicit metric on the space of locally finite graphexes; in fact, it does not even address the question of when two graphexes are equivalent in the sense that the resulting point processes are equal in law.

To discuss the second question, we define measure-preserving transformations, pullbacks, and couplings. Given two $\sigma$-finite spaces $\boldsymbol{\Omega}=(\Omega, \mathcal{F}, \mu)$ and $\boldsymbol{\Omega}^{\prime}=$ $\left(\Omega^{\prime}, \mathcal{F}^{\prime}, \mu^{\prime}\right)$, we say that a map $\phi: \Omega^{\prime} \rightarrow \Omega$ is a measure-preserving transformation if $\phi$ is measurable and $\mu^{\prime}\left(\phi^{-1}(A)\right)=\mu(A)$ for all $A \in \mathcal{F}$. If $\mathbb{W}=(W, S, I, \boldsymbol{\Omega})$ is a signed graphex over $\boldsymbol{\Omega}$, we define its pullback under $\phi$ to be the graphex $\mathbb{W}^{\phi}=\left(W^{\phi}, S^{\phi}, I, \boldsymbol{\Omega}^{\prime}\right)$ where $W^{\phi}\left(x^{\prime}, y^{\prime}\right)=W\left(\phi\left(x^{\prime}\right), \phi\left(x^{\prime}\right)\right)$ and $S^{\phi}\left(x^{\prime}\right)=S(\phi(x))$. It is clear that for unsigned graphexes $\mathbb{W}$ and $\mathbb{W}^{\phi}$ give rise to the same process of random graphs. Note that we can define the pullback even when $\varphi$ is measurable but not measure-preserving, but in this case the two graphexes do not necessarily give rise to the same random process. Nevertheless, we will sometimes use pullbacks in this situation. If we do, we will write $\mathbb{W}^{\varphi, \mu^{\prime}}$ to emphasize the dependence on the measure on $\Omega^{\prime}$. Given two $\sigma$-finite spaces $\boldsymbol{\Omega}_{1}=\left(\Omega_{1}, \mathcal{F}_{1}, \mu_{1}\right)$ and $\boldsymbol{\Omega}_{2}=\left(\Omega_{2}, \mathcal{F}_{2}, \mu_{2}\right)$, 
we say that $\mu$ is a coupling of $\mu_{1}$ and $\mu_{2}$ if $\mu$ is a measure on $\mathcal{F}_{1} \times \mathcal{F}_{2}$ such that $\mu\left(\Omega_{1} \times S_{2}\right)=\mu_{2}\left(S_{2}\right)$ and $\mu\left(S_{1} \times \Omega_{2}\right)=\mu_{1}\left(S_{1}\right)$ for all $S_{1} \in \mathcal{F}_{1}$ and all $S_{2} \in \mathcal{F}_{2}$. Note that the existence of such a coupling implies that $\mu_{1}\left(\Omega_{1}\right)=\mu\left(\Omega_{1} \times \Omega_{2}\right)=\mu_{2}\left(\Omega_{2}\right)$. It turns that this condition is both necessary and sufficient for the existence of a coupling; see [3] for a proof.

Based on the known results for dense graphs, one might conjecture that two graphexes are equivalent if and only if there exists a third graphex such that both are pullbacks of this third graphex. It turns out that this is not quite correct, but that it is correct once we remove the part of the underlying space on which $D_{\mathbb{W}}=0$. This is the statement of the following theorem, which is one of the main results of this paper, and will be proved in Section 8.

Theorem 2.5. Let $\mathbb{W}_{1}=\left(W_{1}, S_{1}, I_{1}, \boldsymbol{\Omega}_{1}\right)$ and $\mathbb{W}_{2}=\left(W_{2}, S_{2}, I_{2}, \boldsymbol{\Omega}_{2}\right)$ be graphexes, where $\boldsymbol{\Omega}_{i}=\left(\Omega_{i}, \mathcal{F}_{i}, \mu_{i}\right)$ are $\sigma$-finite spaces. Then $G_{t}\left(\mathbb{W}_{1}\right)$ and $G_{t}\left(\mathbb{W}_{2}\right)$ have the same distribution for all $t \in \mathbb{R}_{+}$if and only if there exists a third graphex $\mathbb{W}=$ $(W, S, I, \boldsymbol{\Omega})$ over a $\sigma$-finite measure space $\boldsymbol{\Omega}=(\Omega, \mathcal{F}, \mu)$ and measure preserving maps $\phi_{i}: \operatorname{dsupp} \mathbb{W}_{i} \rightarrow \Omega$ such that $\left.\mathbb{W}_{i}\right|_{\text {dsupp } \mathbb{W}_{i}}=\mathbb{W}^{\phi_{i}}$ almost everywhere.

Remark 2.6. If the two graphexes are defined over Borel spaces, we can prove an analogous statement where the measure-preserving maps are turned around. Specifically, for the case where $\boldsymbol{\Omega}_{1}$ and $\boldsymbol{\Omega}_{2}$ are $\sigma$-finite Borel spaces, we can prove that $G_{t}\left(\mathbb{W}_{1}\right)$ and $G_{t}\left(\mathbb{W}_{2}\right)$ have the same distribution for all $t \in \mathbb{R}_{+}$if and only if there exists a $\sigma$-finite Borel space $\boldsymbol{\Omega}=(\Omega, \mathcal{F}, \mu)$ and measure preserving maps $\pi_{i}: \Omega \rightarrow \operatorname{dsupp} \mathbb{W}_{i}$ such that $\left(\left.\mathbb{W}_{1}\right|_{\text {dsupp } \mathbb{W}_{1}}\right)^{\pi_{1}}=\left(\left.\mathbb{W}_{2}\right|_{\text {dsupp } \mathbb{W}_{2}}\right)^{\pi_{2}}$ almost everywhere. In this case, the space $\boldsymbol{\Omega}$ can be chosen to be a coupling of $\boldsymbol{\Omega}_{1}$ and $\boldsymbol{\Omega}_{2}$, with $\pi_{i}$ being the coordinate projections from $\Omega_{1} \times \Omega_{2}$ to $\Omega_{i}$. See Theorem 8.2 in Section 8 . For graphexes without a dust and star part, this was independently established in [3] (using a different proof); see also [17], which establishes a similar result (with yet another proof), this time giving a coupling of the two graphexes (again without dust and star part) after trivially extending them rather than restricting them to the support of their marginals.

To address the first question, concerning the relationship between graphexes and the point processes generated by them, we would like to define an analogue of the cut distance for graphons between graphexes, so that two graphexes are close if and only if their graphex processes are close. To this end, we first define some norms of a function $U$ over $\Omega_{1} \times \Omega_{2}$ for two $\sigma$-finite spaces $\boldsymbol{\Omega}_{1}=\left(\Omega_{1}, \mathcal{F}_{1}, \mu_{1}\right)$ and $\Omega_{2}=\left(\Omega_{2}, \mathcal{F}_{2}, \mu_{2}\right)$. We denote by $\|U\|_{p}$ the $L^{p}$ norm of $U$ as a function over $\Omega_{1} \times \Omega_{2}$ (so we forget the product structure). Given two measurable functions $f: \Omega_{1} \rightarrow \mathbb{R}$ and $g: \Omega_{2} \rightarrow \mathbb{R}$, let

$$
\begin{aligned}
& f \circ U(y)=\int_{\Omega_{1}} f(x) U(x, y) d y \\
& U \circ g(x)=\int_{\Omega_{2}} U(x, y) g(y) d y
\end{aligned}
$$

and

$$
f \circ U \circ g=\int_{\Omega_{1} \times \Omega_{2}} f(x) U(x, y) g(y) d y .
$$

We will also use the notation $U_{x}$ for the function $y \mapsto U(x, y)$. 
Definition 2.7. Given a function $U$ defined on $\Omega_{1} \times \Omega_{2}$ for two $\sigma$-finite measure spaces $\boldsymbol{\Omega}_{i}=\left(\Omega_{i}, \mathcal{F}_{i}, \Omega_{i}\right)$ for $i=1,2$, we define

$$
\|U\|_{2 \rightarrow 2}=\sup _{f, g:\|f\|_{2}=\|g\|_{2}=1} f \circ U \circ g=\sup _{g:\|g\|_{2}=1}\|U \circ g\|_{2} .
$$

Note that the norm $\|U\|_{2 \rightarrow 2}$ is simply the operator norm when we consider $U$ the kernel of an operator $\widehat{U}$ from $L^{2}\left(\boldsymbol{\Omega}_{2}\right)$ to $L^{2}\left(\boldsymbol{\Omega}_{1}\right)$. We will therefore call it the kernel norm of $U$. Our next norm is a modification of the standard cut norm; in the dense graph setting, it was first systematically used in [20], where it was defined as a norm for functions defined over a probability space.

Definition 2.8. Given a measurable function $U$ defined on $\Omega \times \Omega$ for a $\sigma$-finite measure space $\Omega$, we define the jumble norm

$$
\|U\|_{\otimes}=\sup _{S, T \subseteq \Omega}\left|\frac{1}{\sqrt{\mu(S) \mu(T)}} \int_{S \times T} U(x, y) d \mu(x) d \mu(y)\right| .
$$

Here the supremum is over subsets with finite and nonzero measure.

It is easy to show that these are norms; in particular, they satisfy the triangle inequality, and are equal to 0 if and only if $U$ is zero almost everywhere. If we want to stress the dependence of these norms on the measure $\mu$ and the function $U$, we write $\|U\|_{*, \mu}$ instead of $\|U\|_{*}$, where $*$ is replaced by the appropriate norm.

We will see later that for graphexes with uniformly bounded marginals and uniformly bounded $\|\cdot\|_{1}$ norms, the $\|\cdot\|_{2 \rightarrow 2}$ norm and the $\|\cdot\|_{\otimes}$ norm are equivalent (Lemma 3.22), implying in particular that they are equivalent in the theory of dense graph limits (where $\Omega$ has bounded measure). In the dense setting, the above two norms are also equivalent to the standard cut norm, defined as

$$
\|U\|_{\square}=\sup _{S, T \subseteq \Omega}\left|\int_{S \times T} U(x, y) d \mu(x) d \mu(y)\right|=\sup _{f, g: \Omega \rightarrow[0,1]}|f \circ U \circ g| .
$$

Indeed, $\|U\|_{\square} \leq\|U\|_{\otimes} \mu(\Omega)$ and $\|U\|_{\otimes} \leq \sqrt{\|U\|_{\square}\|U\|_{\infty}}$, where the second bound follows from the fact that

$$
\left|\int_{S \times T} U(x, y) d \mu(x) d \mu(y)\right| \leq \inf \left\{\lambda(S) \lambda(T)\|U\|_{\infty},\|U\|_{\square}\right\} .
$$

Therefore, in the theory of dense graph limits all three norms are equivalent. However, although the cut norm is the simplest to state, we believe that the kernel norm $\|\cdot\|_{2 \rightarrow 2}$ norm is the correct extension to graphexes.

We now define some distances between graphexes. First, we define the $\delta_{2 \rightarrow 2}$ distance, which will define a notion of convergence that is equivalent to GP-convergence for graphexes with uniformly bounded marginals. The definition of $\delta_{2 \rightarrow 2}$ will make sense for signed graphexes, provided both the graphon parts and the absolute marginals are in $L^{1} \cap L^{2}$. We will therefore define the $\delta_{2 \rightarrow 2}$ metric in this more general ${ }^{1}$ setting.

\footnotetext{
${ }^{1}$ To see that this setting is indeed more general than the assumption of bounded marginals for (unsigned) graphexes we recall that by Proposition 2.4, a graphex with bounded marginals is integrable. Using this, and the fact that by definition, the graphon part of a graphex is bounded, the claim is easy to verify.
} 
Definition 2.9. A signed graphex $\mathbb{W}=(W, S, I, \boldsymbol{\Omega})$ over $\boldsymbol{\Omega}=(\Omega, \mathcal{F}, \mu)$ is said to be in $L^{1} \cap L^{2}$ if both $W$ and $\left.D\right|_{\mathbb{W} \mid}$ are in $L^{1} \cap L^{2}$. Here $|\mathbb{W}|$ is the graphex $|\mathbb{W}|=(|W|,|S|,|I|, \boldsymbol{\Omega})$.

Suppose $\mathbb{W}_{1}=\left(W_{1}, S_{1}, I_{1}, \boldsymbol{\Omega}\right)$ and $\mathbb{W}_{2}=\left(W_{2}, S_{2}, I_{2}, \boldsymbol{\Omega}\right)$ are defined on the same underlying space $\boldsymbol{\Omega}$. We then define their $d_{2 \rightarrow 2^{-}}$-distance as

$$
d_{2 \rightarrow 2}\left(\mathbb{W}_{1}, \mathbb{W}_{2}\right)=\max \left(\left\|W_{1}-W_{2}\right\|_{2 \rightarrow 2}, \sqrt{\left\|D_{\mathbb{W}_{1}}-D_{\mathbb{W}_{2}}\right\|_{2}}, \sqrt[3]{\left|\rho\left(\mathbb{W}_{1}\right)-\rho\left(\mathbb{W}_{2}\right)\right|}\right),
$$

where $\rho\left(\mathbb{W}_{i}\right)$ is the "edge density" of the signed graphex $\mathbb{W}_{i}$,

$$
\rho\left(\mathbb{W}_{i}\right)=\int W_{i}+2 \int S_{i}+2 I .
$$

The reason we take the roots will become clearer later when we define the general distance $\delta_{\diamond}$. Since $\sqrt{c_{1}+c_{2}} \leq \sqrt{c_{1}}+\sqrt{c_{2}}$ and $\sqrt[3]{c_{1}+c_{2}} \leq \sqrt[3]{c_{1}}+\sqrt[3]{c_{2}}$, this is indeed a metric.

Next, suppose two signed graphexes in $L^{1} \cap L^{2}, \mathbb{W}_{1}=\left(W_{1}, S_{1}, I_{1}, \Omega_{1}\right)$ and $\mathbb{W}_{2}=\left(W_{2}, S_{2}, I_{2}, \boldsymbol{\Omega}_{2}\right)$, are defined over two $\sigma$-finite spaces $\boldsymbol{\Omega}_{1}=\left(\Omega_{1}, \mathcal{F}_{1}, \mu_{1}\right)$ and $\boldsymbol{\Omega}_{2}=\left(\Omega_{2}, \mathcal{F}_{2}, \mu_{2}\right)$ with $\mu_{1}\left(\Omega_{1}\right)=\mu_{2}\left(\Omega_{2}\right)$. Let $\pi_{1}: \Omega_{1} \times \Omega_{2} \rightarrow \Omega_{1}$ and $\pi_{2}: \Omega_{1} \times \Omega_{2} \rightarrow$ $\Omega_{2}$ be the projections. Then we define $\widetilde{\delta}_{2 \rightarrow 2}\left(\mathbb{W}_{1}, \mathbb{W}_{2}\right)$ as the infimum

$$
\widetilde{\delta}_{2 \rightarrow 2}\left(\mathbb{W}_{1}, \mathbb{W}_{2}\right)=\inf _{\mu} d_{2 \rightarrow 2}\left(\mathbb{W}_{1}^{\pi_{1}, \mu}, \mathbb{W}_{2}^{\pi_{2}, \mu}\right),
$$

where the infimum is over all couplings $\mu$ of $\mu_{1}$ and $\mu_{2}$.

To define the $\delta_{2 \rightarrow 2}$-distance we need one more notion, that of a trivial extension of $\mathbb{W}=(W, S, I, \boldsymbol{\Omega})$, where $\boldsymbol{\Omega}=(\Omega, \mathcal{F}, \mu)$ is a $\sigma$-finite measure space. It is defined as a quadruple $\mathbb{W}^{\prime}=\left(W^{\prime}, S^{\prime}, I^{\prime}, \boldsymbol{\Omega}^{\prime}\right)$ where $\boldsymbol{\Omega}^{\prime}=\left(\Omega^{\prime}, \mathcal{F}^{\prime}, \mu^{\prime}\right)$ is a $\sigma$-finite measure space such that $\Omega \in \mathcal{F}^{\prime}, \mathcal{F}=\left\{A \in \mathcal{F}^{\prime}: A \subseteq \Omega\right\}$, and $\mu$ is the restriction of $\mu^{\prime}$ to $\mathcal{F}$, while $W^{\prime}$ is the extension of $W$ that is 0 on the complement of $\Omega \times \Omega, S^{\prime}$ is the extension of $S$ that is 0 on the complement of $\Omega$, and $I^{\prime}=I$. It is easy to see that taking a trivial extension of a graphex has no effect on $\mathcal{G}_{T}$ or $\mathcal{G}_{\infty}$ (since Poisson points sampled in the complement of $\Omega$ will be isolated for all $T$ ).

Definition 2.10. Let $\mathbb{W}_{1}$ and $\mathbb{W}_{2}$ be signed graphexes in $L^{1} \cap L^{2}$. Then we define

$$
\delta_{2 \rightarrow 2}\left(\mathbb{W}_{1}, \mathbb{W}_{2}\right)=\widetilde{\delta}_{2 \rightarrow 2}\left(\mathbb{W}_{1}^{\prime}, \mathbb{W}_{2}^{\prime}\right),
$$

where $\mathbb{W}_{1}^{\prime}$ and $\mathbb{W}_{2}^{\prime}$ are trivial extensions of $\mathbb{W}_{1}$ and $\mathbb{W}_{2}$ to measure spaces of infinite total mass. We refer to $\delta_{2 \rightarrow 2}\left(\mathbb{W}_{1}, \mathbb{W}_{2}\right)$ as the kernel distance of $\mathbb{W}_{1}$ and $\mathbb{W}_{2}$ and call $\delta_{2 \rightarrow 2}$ the kernel metric.

The existence of these extensions is trivial, since we can always append an interval equipped with the Lebesgue measure. Nevertheless, it is not clear that $\delta_{2 \rightarrow 2}\left(\mathbb{W}_{1}, \mathbb{W}_{2}\right)$ is well defined, since the right side of (2.4) could depend on the particular choice of the extensions $\mathbb{W}_{1}^{\prime}$ and $\mathbb{W}_{2}^{\prime}$. In a similar way, while it is clear that $\delta_{2 \rightarrow 2}$ is symmetric and that $\delta_{2 \rightarrow 2}(\mathbb{W}, \mathbb{W})=0$, it is not clear that it is a metric (even after factoring out the null space), since it is not clear that it satisfies the triangle inequality. The following theorem addresses both questions, and will be proved in Section 3.

Theorem 2.11. Let $\mathbb{W}_{1}$ and $\mathbb{W}_{2}$ be signed graphexes in $L^{1} \cap L^{2}$. Then the right side of (2.4) does not depend on the choice of the trivial extensions $\mathbb{W}_{1}^{\prime}$ and $\mathbb{W}_{2}^{\prime}$. Furthermore, given three signed graphexes $\mathbb{W}_{1}, \mathbb{W}_{2}, \mathbb{W}_{3}$ in $L^{1} \cap L^{2}$,

$$
\delta_{2 \rightarrow 2}\left(\mathbb{W}_{1}, \mathbb{W}_{3}\right) \leq \delta_{2 \rightarrow 2}\left(\mathbb{W}_{1}, \mathbb{W}_{2}\right)+\delta_{2 \rightarrow 2}\left(\mathbb{W}_{2}, \mathbb{W}_{3}\right) \text {. }
$$


Therefore, $\delta_{2 \rightarrow 2}$ is a well-defined pseudometric.

Remark 2.12. In [3], when defining the cut distance between two graphons, it was only necessary to extend the smaller space to the larger one, and it was not necessary to extend further. It is natural to ask whether a trivial extension to a space of infinite metric is necessary, or, equivalently, whether for two graphexes $\mathbb{W}_{1}, \mathbb{W}_{2}$ defined on spaces with the same (finite) measure, $\widetilde{\delta}_{2 \rightarrow 2}\left(\mathbb{W}_{1}, \mathbb{W}_{2}\right)=\delta_{2 \rightarrow 2}\left(\mathbb{W}_{1}, \mathbb{W}_{2}\right)$. In contrast to the cut distance discussed in [3], for the kernel metric it is sometimes necessary to take trivial extensions of both spaces, not just an extension of the smaller space to one of the same measure as the larger one. See Example 3.11 in Section 3.

Our next theorem states that on sets with uniformly bounded marginals, the topology induced by the kernel metric $\delta_{2 \rightarrow 2}$ is equivalent to the topology of GPconvergence. We will prove it in Sections 6 and 7 .

Theorem 2.13. For any $D>0, \delta_{2 \rightarrow 2}$-convergence is equivalent to GP-convergence on the space of graphexes with D-bounded marginals.

In general, $\delta_{2 \rightarrow 2^{-}}$-convergence implies GP-convergence, but the reverse is not true. This is because if we do not assume bounded marginals, it is possible to have a very small measure set with very large degree. This will have a non-negligible effect on $\delta_{2 \rightarrow 2}$ distance; however, for a fixed $T$, the chances of obtaining a vertex in the small set is small, and thus has a small effect on sampling. To give a more concrete example, let $W_{n}$ be equal to 1 on $[0,1 / n] \times[1,1+n]$ and $[1,1+n] \times[0,1 / n]$, and zero everywhere else. Let $\mathbb{W}_{n}=\left(W_{n}, 0,0, \mathbb{R}_{+}\right)$. Then for any fixed $T$, the probability of seeing a single edge in $G_{T}\left(\mathbb{W}_{n}\right)$ converges to 0 , and therefore $\mathbb{W}_{n}$ is GP-convergent to 0 . However, it is easy to see that $\delta_{2 \rightarrow 2}\left(\mathbb{W}_{n}, 0\right)$ does not converge to 0 . To address this issue, we will define a new distance such that two graphexes whose graphex processes can be obtained from each other by removing a small set of vertices are close in the new metric. Our construction is loosely motivated by the construction of the usual metric of weak convergence. For that reason, we will refer to the new metric as the weak kernel metric.

Before defining this distance, we introduce the notation $\mu-r \leq \mu^{\prime} \leq \mu$ whenever $\mu, \mu^{\prime}$ are two measures over the same measurable space $(\Omega, \mathcal{F})$ such that

$$
\mu(B)-r \leq \mu^{\prime}(B) \leq \mu(B)
$$

for all measurable sets $B$. Note that this property is equivalent to the existence of a function $h: \Omega \rightarrow[0,1]$ such that $\mu^{\prime}(B)=\int_{B} h d \mu$ and $\|1-h\|_{1, \mu} \leq r$. An example of such a function, which we will often use, is the indicator function of a set $\Omega^{\prime} \subseteq \Omega$ such that $\mu\left(\Omega \backslash \Omega^{\prime}\right) \leq r$.

We will define the weak kernel metric for arbitrary graphexes (removing the condition that they are in $L^{1} \cap L^{2}$ ), and in fact will again allow for signed graphexes. We will assume that the graphon parts of these signed graphexes are bounded in the $L^{\infty}$ norm, a condition which is true for unsigned graphexes, since for these, the graphon part takes values in $[0,1]$.

Definition 2.14. Let $\mathbb{W}_{1}=\left(W_{1}, S_{1}, I_{1}, \boldsymbol{\Omega}_{1}\right)$ and $\mathbb{W}_{2}=\left(W_{2}, S_{2}, I_{2}, \boldsymbol{\Omega}_{2}\right)$ be signed graphexes, where $\boldsymbol{\Omega}_{i}=\left(\Omega_{i}, \mathcal{F}_{i}, \mu_{i}\right)$ and $\left\|W_{i}\right\|_{\infty}<\infty$ for $i=1,2$. We define $\delta_{\diamond}\left(\mathbb{W}_{1}, \mathbb{W}_{2}\right)$ as the infimum of the set of real numbers $c$ such that there exist two measures $\widetilde{\mu}_{1}$ and $\widetilde{\mu}_{2}$ over $\left(\Omega_{1}, \mathcal{F}_{1}\right)$ and $\left(\Omega_{2}, \mathcal{F}_{2}\right)$ that satisfy the following: the signed 
graphexes $\widetilde{\mathbb{W}}_{1}$ and $\widetilde{\mathbb{W}}_{2}$ obtained from $\mathbb{W}_{1}$ and $\mathbb{W}_{2}$ by replacing $\mu_{1}$ and $\mu_{2}$ by $\widetilde{\mu}_{1}$ and $\widetilde{\mu}_{2}$, respectively, are in $L^{1} \cap L^{2}$, and

(1) for $i=1,2$, we have $\mu_{i}-c^{2} \leq \widetilde{\mu}_{i} \leq \mu_{i}$, and

(2) $\delta_{2 \rightarrow 2}\left(\widetilde{\mathbb{W}}_{1}, \widetilde{\mathbb{W}}_{2}\right) \leq c$.

We refer to $\delta_{\diamond}\left(\mathbb{W}_{1}, \mathbb{W}_{2}\right)$ as the weak kernel distance between $\mathbb{W}_{1}$ and $\mathbb{W}_{2}$ and call $\delta_{\diamond}$ the weak kernel metric.

Note that for unsigned graphexes, the weak kernel distance is well defined and finite. Indeed, given $0<D<\infty$, choose $\widetilde{\mu}_{i}$ as the restriction of $\mu_{i}$ to $\left\{D_{\mathbb{W}_{i}} \leq D\right\}$. Proposition 2.4 then implies that $\left\{D_{\mathbb{W}_{i}}>D\right\}$ has finite measure, and $\left.\mathbb{W}_{i}\right|_{\left\{D_{\mathbb{W}_{i}} \leq D\right\}}$ is integrable and hence in $L^{1} \cap L^{2}$. The fact that $\delta_{\diamond}\left(\mathbb{W}_{1}, \mathbb{W}_{2}\right)$ is well defined for signed graphexes with bounded graphon part follows from Proposition 2.4 and further arguments, and is deferred to Section 3; see in particular Lemma 3.12 in that section.

We will show that $\delta_{\diamond}$ is a pseudometric. It is clear that it is symmetric, and that $\delta_{\diamond}(\mathbb{W}, \mathbb{W})=0$. It is not obvious that it satisfies the triangle inequality. We will prove this fact in Section 3.

Theorem 2.15. Given three signed graphexes $\mathbb{W}_{1}, \mathbb{W}_{2}, \mathbb{W}_{3}$ with bounded graphon part,

$$
\delta_{\diamond}\left(\mathbb{W}_{1}, \mathbb{W}_{3}\right) \leq \delta_{\diamond}\left(\mathbb{W}_{1}, \mathbb{W}_{2}\right)+\delta_{\diamond}\left(\mathbb{W}_{2}, \mathbb{W}_{3}\right)
$$

Therefore, $\delta_{\diamond}$ is a pseudometric.

Remark 2.16. Given a signed graphex $\mathbb{W}=(W, S, I, \boldsymbol{\Omega})$ with $\boldsymbol{\Omega}=(\Omega, \mathcal{F}, \mu)$ and a measure-preserving map $\varphi: \boldsymbol{\Omega}^{\prime} \rightarrow \boldsymbol{\Omega}$, let $\mathbb{W}^{\prime}=\mathbb{W}^{\varphi}$ almost everywhere. We can take a coupling $\widetilde{\mu}$ on $\boldsymbol{\Omega}^{\prime} \times \boldsymbol{\Omega}$ defined by $\widetilde{\mu}(A \times B)=\mu^{\prime}\left(A \cap \varphi^{-1}(B)\right)$. It is easy to see that then the pullbacks of the two signed graphexes to $\boldsymbol{\Omega}^{\prime} \times \Omega$ will be equal almost everywhere, which implies that $\delta_{2 \rightarrow 2}\left(\mathbb{W}, \mathbb{W}^{\prime}\right)=\widetilde{\delta}_{2 \rightarrow 2}\left(\mathbb{W}^{\prime}, \mathbb{W}\right)=\delta_{\diamond}\left(\mathbb{W}^{\prime}, \mathbb{W}\right)=\widetilde{\delta}_{\diamond}(\mathbb{W}, \mathbb{W})=0$.

With this new metric, we now have a definition of distance for any pair of graphexes. Note that in general, the metrics $\delta_{2 \rightarrow 2}$ and $\delta_{\diamond}$ are not be the same, even if both are finite. However, we will show that for graphexes with uniformly bounded marginals, the two metrics provide the same topology. This is the content of the next proposition, which will be proved in Section 3.

Proposition 2.17. Fix $D<\infty$. Then $\delta_{\diamond}$ and $\delta_{2 \rightarrow 2}$ give an equivalent topology on the space of graphexes with D-bounded marginals.

We will also show that convergence in the weak kernel metric $\delta_{\diamond}$ is indeed equivalent to GP-convergence. This is the statement of the next theorem, and is one of the two main results of this paper. It will be proved using three main ingredients: a compactness statement stemming from a suitable analogue of the Frieze-Kannan regularity lemma, a counting lemma showing that subgraph counts in the graphs $G_{T}(\mathbb{W})$ are close if the corresponding graphexes are close in the metric $\delta_{2 \rightarrow 2}$ (and the graphexes have uniformly bounded marginals), and a sampling lemma showing that as $T \rightarrow \infty$, the suitably rescaled graphex process $G_{T}(\mathbb{W})$ converges to $\mathbb{W}$ in probability. These techniques are developed in Sections 5, 6, and 7, and are combined to prove the theorem at the end of Section 7 , where we will also prove Theorem 2.13.

Theorem 2.18. Given a sequence of graphexes $\mathbb{W}_{n}$ and a graphex $\mathbb{W}, \mathbb{W}_{n}$ is $G P$-convergent to $\mathbb{W}$ if and only if $\delta_{\diamond}\left(\mathbb{W}_{n}, \mathbb{W}\right) \rightarrow 0$. 
Remark 2.19. The reader might wonder whether instead of building our metric for GP-convergence around the kernel norm $\|\cdot\|_{2 \rightarrow 2}$, one could equivalently build it around the cut norm, $\|\cdot\|_{\square}$. Concretely, one might want to define $d_{\square}$ by replacing the kernel norm in (2.1) by the cut norm and the $L^{2}$ norm by the $L^{1}$ norm, then proceed as in (2.3) and (2.4) to obtain a cut distance $\delta_{\square}$ between graphexes with bounded marginals, and finally proceed as in Definition 2.14 to obtain a "weak cut metric" for arbitrary graphexes.

The following example shows that this approach does not work, in that it will not metricize GP-convergence. Define $W_{n}$ to be the graphex that is constant and equal to $n^{-2}$ over $[0, n]^{2}$ and 0 everywhere else, and set $\mathbb{W}_{n}=\left(W_{n}, 0,0, \mathbb{R}_{+}\right)$, where $\mathbb{R}_{+}$is equipped with the Lebesgue measure. The marginal $D_{\mathbb{W}_{n}}$ of $\mathbb{W}_{n}$ is then equal to $1 / n$ times the indicator function of the interval $[0, n]$, and its $L^{1}$ norm is equal to 1 . It is then not hard to check that $\mathbb{W}_{n}$ converges to the pure dust graphex $\left(0,0,1, \mathbb{R}_{+}\right)$in the metric $\delta_{\diamond}$. Indeed, $\left\|W_{n}\right\|_{2 \rightarrow 2} \rightarrow 0$ and $\left\|D_{\mathbb{W}_{n}}\right\|_{2}=n^{-1 / 2} \rightarrow 0$, while $\left\|\mathbb{W}_{n}\right\|=1 \rightarrow 1=\|\mathbb{W}\|_{1}$, which immediately implies convergence in the metric $\delta_{\diamond}$ and hence GP-convergence (based on the proof of equivalence in this paper, though for this specific case it is simple to check GP-convergence directly). By contrast, $\left\|W_{n}\right\|_{\square}=\left\|W_{n}\right\|_{1}=1$ stays bounded away from zero, showing in particular that $\mathbb{W}_{n}$ does not converge to $\mathbb{W}$ in the cut metric $\delta_{\square}$. Since changing the Lebesgue measure to a measure $\mu_{n}$ such that $\lambda-\varepsilon_{n} \leq \mu_{n} \leq \lambda$ with $\varepsilon_{n} \rightarrow 0$ will asymptotically not change the cut norm of $W_{n}$, the graphexes $\mathbb{W}_{n}$ do not converge to $\mathbb{W}$ in the weak cut metric either. Note that this can't be cured by choosing a different norm for the marginal difference $D_{\mathbb{W}_{1}}-D_{\mathbb{W}_{2}}$, e.g., by keeping the $L^{2}$ norm for that part, since the above counter example works independently of the norm used for that part.

In studying the general topology of graphexes, we define a notion of tightness for sets of graphexes. Tight sets play an important role, in particular, they are the precompact sets in our topology: any sequence that is tight has a convergent subsequence, and any convergent sequence must be tight.

Definition 2.20. A set $\mathcal{S}$ of graphexes is tight if for every $\varepsilon>0$, there exist $C$ and $D$ such that for every $\mathbb{W} \in \mathcal{S}, \mathbb{W}=(W, S, I, \boldsymbol{\Omega})$ with $\boldsymbol{\Omega}=(\Omega, \mathcal{F}, \mu)$, there exists $\Omega_{\varepsilon} \subseteq \Omega$ such that $\mu\left(\Omega_{\varepsilon}\right) \leq \varepsilon$ and the graphex $\mathbb{W}^{\prime}=\left.\mathbb{W}\right|_{\Omega \backslash \Omega_{\varepsilon}}$ is $(C, D)$-bounded. Here a graphex $\mathbb{W}^{\prime}$ is called $(C, D)$-bounded if its marginals are $D$-bounded and $\left\|\mathbb{W}^{\prime}\right\|_{1} \leq C$.

Note that Proposition 2.4 implies that every finite set of graphexes is tight. In Section 4, we will prove that a set $\mathcal{S}$ of graphexes is tight if and only if for all fixed $T$, the corresponding set $\left\{G_{T}(\mathbb{W})\right\}_{\mathbb{W} \in \mathcal{S}}$ of unlabeled graphex processes at time $T$ is tight (which will also be equivalent to the existence of some $T>0$ such that $\left\{G_{T}(\mathbb{W})\right\}_{\mathbb{W} \in \mathcal{S}}$ is tight; see Theorem 4.1 below). Here, as usual, a collection $\mathscr{S}$ of distributions on finite graphs is called tight if for every $\varepsilon>0$, there exists a finite set $T$ of graphs such that for each of the random graphs in $\mathscr{S}$, the probability that the random graph is not isomorphic to a graph in $T$ is at most $\varepsilon$. This is equivalent to the set of random measures being tight under the discrete topology on the set of isomorphism classes of finite graphs, or the set of distributions of the number of edges being tight.

Our main theorem concerning tightness is the following theorem. It will be proved in Section 5, where we will establish a version of the weak (or Frieze-Kannan) 
regularity lemma for graphexes. Note that while our regularity lemma will hold for signed graphexes, the following is only stated for unsigned graphexes. The reason is that our proof relies heavily on the notion of tightness, which we only develop for unsigned graphexes; see also Remark 4.8 in Section 4.

Theorem 2.21. The space of all graphexes is complete under the topology induced by the weak kernel metric $\delta_{\diamond}$. A subset is relatively compact if and only if it is tight. In particular, for any $C$ and $D$, the set of graphexes with $\|\mathbb{W}\|_{1} \leq C$ is compact under $\delta_{\diamond}$, and the set of $(C, D)$-bounded graphexes is compact under both $\delta_{\diamond}$ and $\delta_{2 \rightarrow 2}$.

Remark 2.22. As mentioned above, we only develop the theory of tightness for unsigned graphexes. In particular, we don't characterize the set of precompact signed graphexes. That notwithstanding, some of our compactness results do hold for signed graphexes. Here we only mention that the analogue of the statement for the set of graphexes with $\|\mathbb{W}\|_{1} \leq C$ holds for signed graphexes as well, provided we restrict the $L^{\infty}$ norm of the graphon part (which by definition is bounded by 1 for unsigned graphexes). To be explicit, any sequence of signed graphexes $\mathbb{W}_{n}=\left(W_{n}, S_{n}, I_{n}, \boldsymbol{\Omega}_{n}\right)$ with $\left\|W_{n}\right\|_{\infty} \leq B$ and $\left\|\mathbb{W}_{n}\right\|_{1} \leq C$ has a subsequence converging to a signed graphex $\mathbb{W}=(W, S, I, \boldsymbol{\Omega})$ with $\|W\|_{\infty} \leq B$ and $\|\mathbb{W}\|_{1} \leq C$. See Remark 5.10 in Section 5 below.

The advantage of $(C, D)$-bounded (unsigned) graphexes is that although there is no a priori bound on the size of $G_{T}(\mathbb{W})$ at any given time $T$, for any finite graph $F$, the expected number of copies of $F$ in $G_{T}(\mathbb{W})$ is finite. Furthermore, it turns out that under the assumption of $(C, D)$-boundedness, if two graphexes have the same subgraph densities, then they are equivalent, i.e., have $\delta_{2 \rightarrow 2}$ distance 0 . In this way, we can heuristically think of these subgraph densities as being analogous to moments of random variables: it is well known that moments determine the distribution of random variables, provided the moments do not grow too quickly.

To make these statements precise, we will define homomorphism densities for a graphex $\mathbb{W}$. To this end, we first consider a finite, labeled graph $F$ and a graphon $W$, and define

$$
t(F, W)=\int_{\Omega^{V(F)}} \prod_{(i, j) \in E(F)} W\left(x_{i}, x_{j}\right) \prod_{i \in V(F)} d \mu\left(x_{i}\right) .
$$

Given a connected multigraph $F=(V, E)$ on $k \geq 2$ vertices with no loops, and a graphex $\mathbb{W}=(W, S, I, \boldsymbol{\Omega})$, we define $t(F, \mathbb{W})$ as follows. First, if $F$ consists of a single edge, we define

$$
t(F, \mathbb{W})=\int_{\Omega^{2}} W(x, y) d \mu(x) d \mu(y)+2 \int_{\Omega} S(x) d \mu(x)+2 I=\rho(\mathbb{W}) .
$$

Otherwise, let $V_{\geq 2}$ be the set of vertices of $F$ with degree at least 2, and for each such vertex $v$, let $d_{1}(v)$ be the number of neighbors of $v$ that have degree 1 . Then

$$
t(F, \mathbb{W})=\int_{\Omega^{V_{\geq 2}}} \prod_{\{v, w\} \in E\left(F\left(V_{\geq 2}\right)\right)} W\left(z_{v}, z_{w}\right) \prod_{v \in V_{\geq 2}} D_{\mathbb{W}}\left(z_{v}\right)^{d_{1}(v)} d \mu\left(z_{v}\right) .
$$


Finally, for any multigraph $F$ with no isolated vertices, let $F_{1}, F_{2}, \ldots, F_{k}$ be the components of $F$. Then we define the homomorphism density of $F$ in $\mathbb{W}$ as

$$
t(F, \mathbb{W})=\prod_{i=1}^{k} t\left(F_{i}, \mathbb{W}\right) .
$$

As we will see in Proposition 3.24, these homomorphism densities are defined in such a way that for a simple graph $F$ and a graphex $\mathbb{W}$, they are equal to the expected number of injective homomorphisms from $F$ into $G_{T}(\mathbb{W})$ times $T^{-|V(F)|}$.

Having defined the subgraph densities $t(F, \mathbb{W})$, we can summarize the main relationship between convergence in the metric $\delta_{2 \rightarrow 2}$, convergence of subgraph counts, and GP-convergence in the following theorem. Its proof will also be given at the end of Section 7.

Theorem 2.23. Assume that $\mathbb{W}$ and $\mathbb{W}_{n}$ for $n \geq 1$ are graphexes whose marginals are $D$-bounded for some finite $D$. Then the following are equivalent.

(1) $\delta_{2 \rightarrow 2}\left(\mathbb{W}_{n}, \mathbb{W}\right) \rightarrow 0$.

(2) For every graph $F$ with no isolated vertices, $t\left(F, \mathbb{W}_{n}\right) \rightarrow t(F, \mathbb{W})$.

(3) For every connected graph $F, t\left(F, \mathbb{W}_{n}\right) \rightarrow t(F, \mathbb{W})$.

(4) $G_{T}\left(\mathbb{W}_{n}\right) \rightarrow G_{T}(\mathbb{W})$ in distribution for every $T$.

(5) $G_{T}\left(\mathbb{W}_{n}\right) \rightarrow G_{T}(\mathbb{W})$ in distribution for some $T$.

Remark 2.24. The above theorem implies in particular that in order to check whether a sequence $\mathbb{W}_{n}$ of graphexes with uniformly bounded marginals is GP-convergent, it is enough to check convergence of $G_{T}\left(\mathbb{W}_{n}\right)$ for a single $T>0$. In a similar way, several other properties of sequences or sets of graphexes can be equivalently stated for all $T>0$ or some $T>0$ (see, in particular, the already mentioned Theorem 4.1 about tightness and Theorem 9.1 about uniform integrability). But for general sequences of graphexes, we do not know whether GP-convergence is equivalent to the convergence of $G_{T}\left(\mathbb{W}_{n}\right)$ for just one $T>0$.

It is instructive to compare our notions of convergence to the notions of graph convergence introduced in [3] and [4]. Before defining these notions, we first introduce the notion of a dilated empirical graphon corresponding to a finite graph $G$. It involves a "dilation parameter" $\rho \in \mathbb{R}_{+}$and is defined as the graphex $\mathbb{W}(G, \rho)$ consisting of a zero dust part, a zero star part, a measure space consisting of the vertex set $V(G)$ where each vertex has measure $\rho$, and a graphon $W(G, \rho)$ which is simply the adjacency matrix of $G$. The usual way to embed graphs into the space of graphons in the dense case corresponds to $\rho=1 /|V(G)|$.

By contrast, in [3], $\rho$ was chosen to be $1 / \sqrt{2|E(G)|}$; the resulting dilated empirical graphon was called the stretched empirical graphon, and a sequence was said to converge in the stretched cut metric if the graphons $W(G, 1 / \sqrt{2|E(G)|})$ converge in the cut metric $\delta_{\square}$. It was then shown that this leads to completeness (every Cauchy sequence has a limit), that convergence implies a certain condition called uniform tail regularity, and that any uniformly tail regular sequence has a convergent subsequence.

The notion of convergence in [4] is slightly different. It does not start from a metric, and instead tries to emulate the notion of subgraph convergence from dense graphs. Roughly speaking, it asks that certain random subgraphs of the graphs in the sequence converge in distribution to some well-defined distribution over finite graphs. More precisely, given a parameter $p \in[0,1]$, define $\operatorname{Smpl}(G, p)$ as 
the unlabelled graph obtained by first taking each vertex i.i.d. with probability $p$, then removing all isolated vertices in the resulting subgraph, and finally discarding all the labels. A sequence $G_{n}$ is then said to be sampling convergent if for all $t>0$, the samples

$$
\operatorname{Smpl}\left(G_{n}, \min \left\{1, t / \sqrt{2\left|E\left(G_{n}\right)\right|}\right\}\right)
$$

converge in distribution. It was then shown that any sequence of finite graphs has a convergent subsequence, and that the limiting distribution can be expressed as $G_{t}(\mathbb{W})$ for some integrable graphex $\mathbb{W}$ with $\|\mathbb{W}\|_{1} \leq 1$. It was also shown that this inequality holds with equality if and only if the sequence has a property called uniform sampling regularity.

It is instructive to relate the results and notions from [4] to those developed in this paper. To this end, we first note that - as already observed in [4] - a sequence of graphs is sampling convergent if and only if the stretched canonical graphexes $\mathbb{W}\left(G_{n}, 1 / \sqrt{2\left|E\left(G_{n}\right)\right|}\right)$ are GP-convergent. Since by definition, the stretched canonical graphex has $L^{1}$ norm 1 , this sequence is tight. By our compactness theorem, Theorem 2.21, it therefore has a convergent subsequence.

To relate some of the other notions and results from [3] and [4] to those of this paper, we introduce a couple of definitions. The first notion is that of uniform integrability. Recall that a set $S$ of random variables with values in $\mathbb{R}$ is called uniformly integrable if for every $\varepsilon>0$, there exists $K \in \mathbb{R}$ such that for every $X \in S$,

$$
\mathbb{E}\left[\left.|X| 1\right|_{X \mid>K}\right]<\varepsilon .
$$

Note that this implies that $\mathbb{E}[|X|] \leq \varepsilon+K$, so the set of random variables consists of integrable variables with uniformly bounded integrals. This motivates the following definition.

Definition 2.25. A set of graphexes $\mathcal{S}$ is called uniformly integrable if the graphexes in $\mathcal{S}$ have uniformly bounded $\|\cdot\|_{1}$-norms, and for every $\varepsilon>0$, there exists a $D$ such that for all $\mathbb{W} \in \mathcal{S},\left\|D_{\mathbb{W}} 1_{D_{\mathbb{W}}>D}\right\|_{1}<\varepsilon$.

As we will see in Theorem 9.1 below, uniform integrability of a set $\mathcal{S}$ of graphexes is equivalent to uniform integrability of the random variables $\left\{E\left(G_{T}(\mathbb{W})\right): \mathbb{W} \in \mathcal{S}\right\}$ for all $T>0$ (which is also equivalent to uniform integrability of this set of random variables for some $T>0)$.

The notion of uniform sampling regularity from [4] is then simply uniform integrability of the stretched empirical graphexes, and the following theorem is a more or less straightforward generalization of Corollary 3.10 in [4], which states that the limiting graphex of a sampling convergent sequence of graphs has norm 1 if and only if it is uniformly sampling regular. We will prove the theorem in Section 9.

Theorem 2.26. Suppose $\mathbb{W}_{n}$ is a sequence of integrable graphexes with uniformly bounded $\|\cdot\|_{1}$-norms that converges to a graphex $\mathbb{W}$ in the weak kernel metric. Then

$$
\|\mathbb{W}\|_{1} \leq \liminf _{n \rightarrow \infty}\left\|\mathbb{W}_{n}\right\|_{1}
$$

In particular, $\mathbb{W}$ is integrable. We furthermore have that

$$
\lim _{n \rightarrow \infty}\left\|\mathbb{W}_{n}\right\|_{1}=\|\mathbb{W}\|_{1}
$$

if and only if the sequence $\mathbb{W}_{n}$ is uniformly integrable. 
Our next set of theorems relates the notion of sampling convergence from [4] to the notion of convergence in the cut metric from [3]. We start by recalling the definition of uniform tail regularity from [3] (see also Lemma 9.3 in Section 9 for other, equivalent definitions).

Definition 2.27 ([3]). Given a set of signed, integrable graphons $\mathcal{S}$, we say that they are uniformly tail regular if for any $\varepsilon>0$, there exists $M$ such that for each $W \in \mathcal{S}$ with the usual notation, there exists $\Omega_{0} \subseteq \Omega$ such that $\mu\left(\Omega_{0}\right) \leq M$ and $\|W\|_{1}-\left\|\left.W\right|_{\Omega_{0}}\right\|_{1} \leq \varepsilon$.

Note that uniform tail regularity is more restrictive than uniform integrability (for the set of graphexes obtained by setting the dust and star part to zero). For sequences of graphs, the corresponding result was shown in [4], but it holds in our more general setting as well, with essentially the same proof; see Lemma 9.2 in Section 9 below. More interestingly, any sequence of graphons that is convergent in cut metric has uniformly regular tails, and any sequence of graphons with uniformly regular tails has a subsequence that converges in cut metric. Motivated by this (which is one of the central results of [3]), here we prove the following.

Theorem 2.28. Given a sequence of integrable graphexes of the form

$$
\mathbb{W}_{n}=\left(W_{n}, 0,0, \boldsymbol{\Omega}_{n}\right)
$$

the following are equivalent.

(1) The sequence $W_{n}$ converges to a graphon $W$ in cut metric.

(2) The sequence $W_{n}$ is uniformly tail regular, and in the weak kernel metric, the sequence $\mathbb{W}_{n}$ converges to a graphex of the form $\mathbb{W}=(W, 0,0, \boldsymbol{\Omega})$.

(3) The sequence $W_{n}$ is uniformly tail regular, and in the weak kernel metric, the sequence $\mathbb{W}_{n}$ converges to some graphex $\mathbb{W}$.

This theorem, as well as our next theorem, will also be proved in Section 9 .

Theorem 2.29. Given a sequence of uniformly integrable graphexes

$$
\mathbb{W}_{n}=\left(W_{n}, S_{n}, I_{n}, \boldsymbol{\Omega}_{n}\right),
$$

which converge to a graphex $\mathbb{W}=(W, S, I, \boldsymbol{\Omega})$ in the weak kernel metric, the following are equivalent.

(1) The graphex $\mathbb{W}$ is of the form $\mathbb{W}=(W, 0,0, \boldsymbol{\Omega})$.

(2) $\left\|S_{n}\right\|_{1} \rightarrow 0$ and $I_{n} \rightarrow 0$, and the sequence of graphons $W_{n}$ has uniformly regular tails.

(3) $\left\|S_{n}\right\|_{1} \rightarrow 0$ and $I_{n} \rightarrow 0$, and the sequence of graphons $W_{n}$ converges in the cut metric.

Recall that a sequence of graphs is sampling convergent if and only if the stretched canonical graphexes are GP-convergent. This fact, Theorem 2.26, and Theorem 2.29 together imply that given a sequence of graphs that is sampling convergent to a graphex of norm one, the sequence converges to a pure graphon if and only if the sequence is uniformly tail regular. We have therefore given a characterization of when the notion of sampling convergence from [4] reduces to the notion of convergence in the stretched cut metric from [3].

Remark 2.30. In the above theorem, the assumption of uniform integrability is necessary. To see this, let $W_{n}$ be the graphon defined by being 1 on the set 
$[0,1 / n] \times[1, n+1]$ and its transpose, and 0 otherwise, and let $\mathbb{W}_{n}=\left(W_{n}, 0,0, \mathbb{R}_{+}\right)$. Then the sequence $\mathbb{W}_{n}$ converges to the zero graphex in the weak kernel metric, which is a pure graphon. However, the sequence is clearly not uniform tail regular (or even uniformly integrable). We can also let $S_{n}(x)=1 / n$ on the set $[0, n]$, and 0 everywhere else. In that case the sequence still converges to 0 in the weak kernel metric, but $\left\|S_{n}\right\|_{1}$ does not converge to 0 .

We close this section by discussing possible extensions of our theory. First, as already discussed in the introduction, it would be natural to extend the theory of graphexes to a theory that naturally generates multi-graphs. Note that a priori, this falls plainly in the framework of exchangeable random measures on $\mathbb{R}_{+}^{2}$ as developed by Kallenberg; in fact, it falls into the framework of exchangeable random counting measures. Generalizing the approach of $[26,3]$, this will give a natural notion of "multi-graphexes" characterizing all exchangeable multi-graphs with vertices labelled by $\mathbb{R}_{+}$. But extending the current work to multi-graphexes is beyond the scope of this paper, in particular given that it would require to generalize at least some of the results from $[26,17,25,18,4]$ to this setting in a first step. See [6] for some very preliminary steps in this direction.

The next extension one might want to consider is the extension of our analytical results (i.e., those of our results which do not refer to the graphex process generated by a graphex) to signed graphexes. In contrast to the theory of cut metric convergence for graphons over $\sigma$-finite measure spaces developed in [3], which works as well for signed, unbounded graphons as for graphons with values in $[0,1]$, here we focused most of the theory of graphex convergence on unsigned graphexes (with graphon parts taking values in $[0,1])$. While several of our technical proofs and results hold for signed graphexes (in particular, all of Section 3, as well as parts of Sections 5 and 6 are formulated in this language), the core analytic concepts and results such as tightness, precompactness, etc., have only been formulated for unsigned graphexes. Indeed, we believe that the generalization of Theorem 2.21 to signed graphexes requires modifications to either our topology or our notions of tightness; see Remark 4.8 below. In a similar way, while the identification theorem of [3] works for signed graphons, our identification theorem requires non-negative graphexes, even though one might conjecture that when stated as a characterization of the equivalence classes under the weak kernel metric, it should hold for signed graphexes as well, at least when suitably formulated.

Finally, one might want to consider graphexes where the graphon part $W$ is unbounded, whether non-negative or signed. For non-negative graphexes, one could, for example, follow the approach in [6] and use such a graphex to generate multi-graphs by adding Pois $\left(W\left(x_{i}, y_{i}\right)\right)$ many edges to a pair of Poisson points with features $x_{i}$ and $x_{j}$, or one could try to generalize the approach of [5] to the setting of graphexes, by taking a decreasing "dilution probability" $p_{t}$, and then connect two Poisson points with features $x_{i}$ and $x_{j}$ with probability $\min \left\{1, p_{t} W\left(x_{i}, x_{j}\right)\right\}$ (see [2] for a related approach). But is far from obvious what the analogue of the weak kernel metric should be, and how to generalize our other results to this setting. As the other open questions discussed here, we leave these questions as open research problems. 


\section{Preliminaries}

In this section, we study the metrics $\delta_{2 \rightarrow 2}$ and $\delta_{\diamond}$. In particular, we will prove Theorems 2.11 and 2.15, as well as Proposition 2.17 relating the two for graphexes with bounded marginals (in fact, we will prove its generalization to signed graphexes, stated as Proposition 3.15 below).

In addition, we study the metric $\delta_{\square}$ obtained from $\delta_{2 \rightarrow 2}$ by replacing $d_{2 \rightarrow 2}$ as defined in (2.1) by

$$
d_{\bigotimes}\left(\mathbb{W}_{1}, \mathbb{W}_{2}\right)=\max \left\{\left\|W_{1}-W_{2}\right\|_{\otimes},\left\|D_{\mathbb{W}_{1}}-D_{\mathbb{W}_{2}}\right\|_{\otimes},\left|\rho\left(\mathbb{W}_{1}\right)-\rho\left(\mathbb{W}_{2}\right)\right|\right\} .
$$

Here we use the norm $\|\cdot\|_{\otimes}$ both for functions from $\Omega^{2} \rightarrow \mathbb{R}$ (see Definition 2.8) and functions from $\Omega \rightarrow \mathbb{R}$, where it is defined as

$$
\|F\|_{\otimes}=\sup _{S \subseteq \Omega}\left|\frac{1}{\sqrt{\mu(S)}} \int_{S} F(x) d \mu(x)\right| .
$$

We will in particular show that the analogue of Theorem 2.11 holds for this metric.

Theorem 3.1. Let $\mathbb{W}_{1}$ and $\mathbb{W}_{2}$ be signed graphexes in $L^{1} \cap L^{2}$. Define $\delta_{\bigotimes}$ by replacing the right side of (2.4) with $\widetilde{\delta}_{\square}$, which in turn is obtained from $\widetilde{\delta}_{2 \rightarrow 2}$ by replacing $d_{2 \rightarrow 2}$ with $d_{\bigotimes}$. Then the value of $\delta_{\bigotimes}\left(\mathbb{W}_{1}, \mathbb{W}_{2}\right)$ does not depend on the choice of the trivial extensions $\mathbb{W}_{1}^{\prime}$ and $\mathbb{W}_{2}^{\prime}$, and $\delta_{\bigotimes}$ obeys the triangle inequality, making it a well-defined pseudometric.

This jumble metric will be particularly useful when establishing the regularity lemma for graphexes, which takes a nicer form when stated in terms of the distance $d_{\bigotimes}$ instead of the distance $d_{2 \rightarrow 2}$, both because of the absence of the various roots, and because the proof of the regularity lemma leads more naturally to bounds in term of $\|\cdot\|_{\otimes}$ rather than $\|\cdot\|_{2 \rightarrow 2}$. To obtain our compactness results for the metric $\delta_{\diamond}$ (which is derived from $d_{2 \rightarrow 2}$ ), we will then need to compare the two. We will do this in Proposition 3.19 and Remark 3.23 below.

We will also establish a simple lemma relating the kernel norm to 4-cycle counts (Lemma 3.22). Finally, we will prove that the homomorphism densities $t(F, \mathbb{W})$ indeed describe the expected number of injective homomorphisms from $F$ into $G_{T}(\mathbb{W})$ (Proposition 3.24).

Except for the last result, all results in this section are as easily derived for signed graphexes as for unsigned graphexes. We therefore formulate everything in this section in the language of signed graphexes. To do so, we need very little extra notation, except for the following.

First, we define the absolute marginal of a signed graphex $\mathbb{W}$ as $D_{|\mathbb{W}|}$, and say that $\mathbb{W}$ has $D$-bounded absolute marginals if $\left\|D_{|\mathbb{W}|}\right\|_{\infty} \leq D$. We say that $\mathbb{W}$ is $(C, D)$-bounded if in addition $\|\mathbb{W}\|_{1} \leq C$. Furthermore, we introduce the notion of $(B, C, D)$-boundedness of a graphex $\mathbb{W}=(W, S, I, \boldsymbol{\Omega})$ by requiring that

$$
\|W\|_{\infty} \leq B, \quad\|\mathbb{W}\|_{1} \leq C, \quad \text { and } \quad\left\|D_{|\mathbb{W}|}\right\|_{\infty} \leq D
$$

and finally, we say that $\mathbb{W}$ has a bounded graphon part if $\|W\|_{\infty}<\infty$.

We use the following standard facts about measure-preserving transformations, which we prove for completeness.

Lemma 3.2. Suppose $\phi: \Omega \rightarrow \Omega^{\prime}$ is a measure preserving map between two $\sigma$-finite measure spaces $(\Omega, \mathcal{F}, \mu)$ and $\left(\Omega^{\prime}, \mathcal{F}^{\prime}, \mu^{\prime}\right)$.

(1) For any $\sigma$-algebra $\mathcal{G} \subseteq \mathcal{F}, L^{1}(\Omega, \mathcal{G}, \mu)$ is a closed subspace in $L^{1}(\Omega, \mathcal{F}, \mu)$. 
(2) If $f \in L^{1}\left(\Omega, \phi^{-1}\left(\mathcal{F}^{\prime}\right), \mu\right)$, then there exists a function $f^{\prime} \in L^{1}\left(\Omega^{\prime}, \mathcal{F}^{\prime}, \mu^{\prime}\right)$ such that $f=f^{\prime \phi}=f^{\prime} \circ \phi$ almost everywhere.

(3) The map $\phi^{*}: L^{1}\left(\Omega^{\prime}, \mathcal{F}^{\prime}, \mu^{\prime}\right) \rightarrow L^{1}\left(\Omega, \phi^{-1}\left(\mathcal{F}^{\prime}\right), \mu\right)$ with $f^{\prime} \mapsto f^{\prime \phi}$ is an isometric isomorphism, implying that $\phi^{*}$ and its inverse are continuous and hence Borel measurable.

Proof. (1) $L^{1}(\Omega, \mathcal{G}, \mu)$ is clearly a subspace of $L^{1}(\Omega, \mathcal{F}, \mu)$. Since they are both Banach spaces, they are both complete. Since $L^{1}(\Omega, \mathcal{G}, \mu) \subseteq L^{1}(\Omega, \mathcal{F}, \mu)$ is an isometric embedding, the only way we can have a complete subset of a complete space is if the subset itself is closed.

(2) The map sending $A \subseteq \Omega$ to $\int_{A} f$ defines a finite, signed measure $\nu$ on $\Omega$, absolutely continuous with respect to $\mu$. This measure pushes forward to a signed measure $\nu^{\prime}$ on $\Omega^{\prime}$. If $B \subseteq \Omega^{\prime}$ has $\mu^{\prime}(B)=0$, then $\mu\left(\phi^{-1}(B)\right)=0$, so $\nu^{\prime}(B)=\nu\left(\phi^{-1}(B)\right)=0$. Therefore $\nu^{\prime}$ is absolutely continuous with respect to $\mu^{\prime}$, so it has a Radon-Nikodym derivative $f^{\prime}$. It is straightforward to check that, since $f$ is $\phi^{-1}\left(\mathcal{F}^{\prime}\right)$ measurable, we have $f=f^{\prime \phi}$ almost everywhere.

(3) This follows from the previous parts.

We will also need the following lemma.

Lemma 3.3. Suppose that $\boldsymbol{\Omega}=(\Omega, \mathcal{F}, \mu)$ with $\mu(\Omega)<\infty$, and suppose that $g \in$ $L^{2}(\Omega)$. Let

$$
S=\sup _{f \in L^{\infty}(\Omega), 0 \leq f \leq 1}\left|\frac{\int_{\Omega} f g d \mu}{\sqrt{\int_{\Omega} f d \mu}}\right| .
$$

Then $S \leq\|g\|_{2}<\infty$, and there exists $X \subseteq \Omega$ such that

$$
S=\frac{\left|\int_{X} g d \mu\right|}{\sqrt{\mu(X)}} .
$$

Note that this expression is the same as taking $f=1_{X}$ in (3.3).

Proof. First, note that if $0 \leq f \leq 1$, then

$$
\left|\frac{\int_{\Omega} f g d \mu}{\sqrt{\int_{\Omega} f d \mu}}\right| \leq \sqrt{\frac{\int_{\Omega} f^{2} d \mu \int_{\Omega} g^{2} d \mu}{\int_{\Omega} f d \mu}}=\|g\|_{2} \sqrt{\frac{\int_{\Omega} f^{2} d \mu}{\int_{\Omega} f d \mu}} \leq\|g\|_{2}<\infty .
$$

Next, we will show that there exists a $\delta>0$ such that in (3.3), it suffices to consider $f$ with $\|f\|_{1} \geq \delta$. Let $\widetilde{\Omega}=\Omega \times[0,1]$ and $\widetilde{\mu}=\mu \times \lambda$, where $\lambda$ is the Lebesgue measure, and let $\widetilde{g}(x, t)=g(x)$. Then the expression in (3.3) is the same as taking the supremum of

$$
\frac{\left|\int_{X} \widetilde{g} d \widetilde{\mu}\right|}{\sqrt{\widetilde{\mu}(X)}}
$$

over $X \subseteq \widetilde{\Omega}$. Indeed, given $X \subseteq \widetilde{\Omega}$, we can plug in the function $f(x)=\lambda(\{t:(x, t) \in$ $X\})$ into (3.3), which is defined almost everywhere, and given $f$ as in (3.3), we can take $X=\{(x, t): t \leq f(x)\}$. It is straightforward to check that (3.3) and the above expression give the same value. Note that we have

$$
\frac{\left|\int_{X} \widetilde{g} d \widetilde{\mu}\right|}{\sqrt{\widetilde{\mu}(X)}} \leq\left\|\left.\widetilde{g}\right|_{X}\right\|_{2}
$$


Since $\|\widetilde{g}\|_{2}<\infty$, for any $\varepsilon>0$, there exists a $K$ such that

$$
\int_{X} \widetilde{g}^{2} d \mu \leq \int \widetilde{g}^{2} 1_{\widetilde{g}>\sqrt{K}}+K \widetilde{\mu}(X) \leq \frac{\varepsilon^{2}}{2}+K \widetilde{\mu}(X) .
$$

Thus, given $\varepsilon>0$, we can find a $\delta>0$ such that if $\mu(X)<\delta$, then $\left\|\left.\widetilde{g}\right|_{X}\right\|_{2} \leq \varepsilon$. Taking $\varepsilon=S / 2$ (note that unless $g=0, S>0$ ), we obtain a $\delta$ such that if $\int_{\Omega} f d \mu<\delta$, then the expression in (3.3) is less than $S / 2$. This means that it suffices to take the supremum over $f$ with $\|f\|_{1} \geq \delta$.

Recall that we assumed that $\Omega$ has finite measure. The set of $f \in L^{\infty}(\Omega)$ with $0 \leq f \leq 1$ and $\|f\|_{1} \geq \delta$ is weak-* closed and therefore weak-* compact. The expression in (3.3) is weak-* continuous; therefore, there exists an $f$ which maximizes the expression.

Clearly such an $f$ is supported either on the set $\{g>0\}$ or $\{g<0\}$. Assume without loss of generality that it is supported on $\{g>0\}$. Suppose that $f$ is not equal to 0 or 1 almost everywhere. Then we can find $0 \leq h \leq \widetilde{h} \leq 1$, supported on $\{g>0\}$, such that $f=(\widetilde{h}+h) / 2$, and it is not the case that $\widetilde{h}=h$ almost everywhere. This implies that

$$
\int_{\Omega}(\widetilde{h}-h) d \mu>0
$$

and

$$
\int_{\Omega}(\widetilde{h}-h) g d \mu>0
$$

Let $h_{t}=t \widetilde{h}+(1-t) h$. Note that for $t \in[0,1], 0 \leq h_{t} \leq 1$ almost everywhere. Let

$$
p(t)=\frac{\int_{\Omega} h_{t} g}{\sqrt{\int_{\Omega} h_{t}}} .
$$

We have

$$
\frac{d}{d t} p(t)=\frac{\int_{\Omega}(\widetilde{h}-h) g d \mu \int_{\Omega}(\widetilde{t h}+(1-t) h) d \mu-\frac{1}{2} \int_{\Omega}(\widetilde{h}-h) d \mu \int_{\Omega}(\widetilde{t h}+(1-t) h) g d \mu}{\left(\int_{\Omega}(\widetilde{t h}+(1-t) h) d \mu\right)^{3 / 2}} .
$$

Notice that the denominator above is always positive, and the numerator above is of the form $A t+B$, where

$$
\begin{aligned}
A & =\int_{\Omega}(\widetilde{h}-h) g d \mu \int_{\Omega}(\widetilde{h}-h) d \mu-\frac{1}{2} \int_{\Omega}(\widetilde{h}-h) d \mu \int_{\Omega}(\widetilde{h}-h) g d \mu \\
& =\frac{1}{2} \int_{\Omega}(\widetilde{h}-h) d \mu \int_{\Omega}(\widetilde{h}-h) g d \mu>0 .
\end{aligned}
$$

This means that there are three possibilities for $\frac{d}{d t} p(t)$ : it can be positive for every $t \in(0,1)$, it can be negative for every $t \in(0,1)$, or it can be negative and then positive. Either of these cases implies that the maximum of $p$ on $[0,1]$ is attained at one or both of the endpoints, and therefore either $p(0)$ or $p(1)$ is strictly greater than $p(1 / 2)$. This contradicts the assumption that $f$ was maximal, completing the proof of the lemma.

Using the previous two lemmas, we prove the following proposition. We will use it for functions which arise as the difference of two graphons with bounded marginals. 
Proposition 3.4. Let $\boldsymbol{\Omega}=(\Omega, \mathcal{F}, \mu)$ and $\boldsymbol{\Omega}^{\prime}=\left(\Omega^{\prime}, \mathcal{F}^{\prime}, \mu^{\prime}\right)$ be $\sigma$-finite measure spaces, and let $\varphi: \Omega^{\prime} \rightarrow \Omega$ be measurable. If $U: \Omega \times \Omega \rightarrow \mathbb{R}$ and $F: \Omega \rightarrow \mathbb{R}$ are square integrable, then

$$
\|U\|_{2 \rightarrow 2}=\left\|U^{\varphi}\right\|_{2 \rightarrow 2}, \quad\|F\|_{\bigotimes}=\left\|F^{\varphi}\right\|_{\bigotimes}, \quad \text { and } \quad\|U\|_{\bigotimes}=\left\|U^{\varphi}\right\|_{\bigotimes} .
$$

If instead of square integrability, we assume that $U$ is integrable, then

$$
\|U\|_{\square}=\left\|U^{\varphi}\right\|_{\square} .
$$

Proof. For any $f, g \in L^{2}(\boldsymbol{\Omega})$, it is easy to see that $f \circ U \circ g=f^{\varphi} \circ U^{\varphi} \circ g^{\varphi}$, and we furthermore have that $f^{\phi}, g^{\phi} \in L^{2}\left(\boldsymbol{\Omega}^{\prime}\right)$ with $\left\|f^{\phi}\right\|_{2}=\|f\|_{2}$ and $\left\|g^{\phi}\right\|_{2}=\|g\|_{2}$. This implies that

$$
\|U\|_{2 \rightarrow 2} \leq\left\|U^{\varphi}\right\|_{2 \rightarrow 2} .
$$

To prove the opposite inequality, let $\widehat{f}, \widehat{g} \in L^{2}\left(\boldsymbol{\Omega}^{\prime}\right)$, and assume first that $\widehat{f}, \widehat{g} \in$ $L^{1}\left(\boldsymbol{\Omega}^{\prime}\right)$ as well. Let $f^{\prime}=\mathbb{E}\left[\widehat{f} \mid \varphi^{-1}(\mathcal{F})\right]$ and $g^{\prime}=\mathbb{E}\left[\widehat{g} \mid \varphi^{-1}(\mathcal{F})\right]$. That is, $f^{\prime}$ is a $\varphi^{-1}(\mathcal{F})$-measurable function such that for any $\varphi^{-1}(\mathcal{F})$-measurable set $S^{\prime} \subseteq \Omega^{\prime}$, $\int_{S^{\prime}} f^{\prime}=\int_{S^{\prime}} \widehat{f}$, and same for $g^{\prime}$. These functions exist by the Radon-Nikodym theorem (since all measures are $\sigma$-finite). Then $\left\|f^{\prime}\right\|_{2} \leq\|\widehat{f}\|_{2},\left\|g^{\prime}\right\|_{2} \leq\|\widehat{g}\|_{2}$. We claim that $f^{\prime} \circ U^{\varphi} \circ g^{\prime}=\widehat{f} \circ U^{\varphi} \circ \widehat{g}$. Indeed, for any $x^{\prime} \in \Omega^{\prime}$,

$$
\left(U^{\varphi}\right)_{x^{\prime}}\left(y^{\prime}\right)=U^{\varphi}\left(x^{\prime}, y^{\prime}\right)=U\left(\varphi\left(x^{\prime}\right), \varphi\left(y^{\prime}\right)\right)=U_{\varphi\left(x^{\prime}\right)}\left(\varphi\left(y^{\prime}\right)\right)=\left(U_{\varphi\left(x^{\prime}\right)}\right)^{\varphi}\left(y^{\prime}\right),
$$

showing that $\left(U^{\varphi}\right)_{x^{\prime}}$ is the pullback of an $\mathcal{F}$-measurable function, and is thus $\varphi^{-1}(\mathcal{F})$-measurable. Therefore, for every $x^{\prime} \in \Omega^{\prime}$,

$$
\int_{\Omega^{\prime}} U^{\varphi}\left(x^{\prime}, y^{\prime}\right) \widehat{g}\left(y^{\prime}\right) d \mu^{\prime}\left(y^{\prime}\right)=\int_{\Omega^{\prime}} U^{\varphi}\left(x^{\prime}, y^{\prime}\right) g^{\prime}\left(y^{\prime}\right) d \mu^{\prime}\left(y^{\prime}\right)
$$

which shows that $\widehat{f} \circ U^{\varphi} \circ \widehat{g}=\widehat{f} \circ U^{\varphi} \circ g^{\prime}$. We can analogously show that $\widehat{f} \circ U^{\varphi} \circ g^{\prime}=$ $f^{\prime} \circ U^{\varphi} \circ g^{\prime}$. Then, since $f^{\prime}$ and $g^{\prime}$ are $\varphi^{-1}(\mathcal{F})$-measurable, there exist by Lemma 3.2 $f, g \in L^{1}(\Omega)$ with $f^{\prime}=f^{\varphi}$ and $g^{\prime}=g^{\varphi}$. This implies that $\|f\|_{2} \leq\|\widehat{f}\|,\|g\|_{2} \leq\|\widehat{g}\|_{2}$, and $f \circ U \circ g=\widehat{f} \circ U^{\varphi} \circ \widehat{g}$, which shows that

$$
\widehat{f} \circ U^{\varphi} \circ \widehat{g} \leq\|U\|_{2 \rightarrow 2}
$$

whenever $\widehat{f}, \widehat{g} \in L^{2}\left(\boldsymbol{\Omega}^{\prime}\right) \cap L^{1}\left(\boldsymbol{\Omega}^{\prime}\right)$ and $\|\widehat{f}\|_{2},\|\widehat{g}\|_{2} \leq 1$. Since $\boldsymbol{\Omega}^{\prime}$ is $\sigma$-finite, any function in $L^{2}\left(\boldsymbol{\Omega}^{\prime}\right)$ can be written as a limit of functions in $L^{2}\left(\boldsymbol{\Omega}^{\prime}\right) \cap L^{1}\left(\boldsymbol{\Omega}^{\prime}\right)$. A dominated convergence argument then shows that the above bound holds whenever $\widehat{f}, \widehat{g} \in L^{2}\left(\boldsymbol{\Omega}^{\prime}\right)$ and $\|\widehat{f}\|_{2},\|\widehat{g}\|_{2} \leq 1$, proving that

$$
\left\|U^{\varphi}\right\|_{2 \rightarrow 2} \leq\|U\|_{2 \rightarrow 2} .
$$

To prove the statement for the cut norm, we use the representation $\|U\|_{\square}=$ $\sup _{f, g: \Omega \rightarrow[0,1]}|f \circ U \circ g|$. Using this representation, the proof for the cut norm proceeds along the same lines as the proof for the $\|\cdot\|_{2 \rightarrow 2}$ norm.

Next, let us prove that $\|F\|_{\otimes}=\left\|F^{\varphi}\right\|_{\bowtie}$. First, for any measurable $X \subseteq \Omega$ with $\mu(X)<\infty$, since $\mu$ is the pushforward of $\mu^{\prime}$,

$$
\frac{\int_{\varphi^{-1}(X)} F^{\varphi} d \mu^{\prime}}{\sqrt{\mu^{\prime}\left(\varphi^{-1}(X)\right)}}=\frac{\int_{X} F d \mu}{\sqrt{\mu(X)}} .
$$


This shows that $\|F\|_{\otimes} \leq\left\|F^{\varphi}\right\|_{\otimes}$. Suppose now that $X^{\prime} \subseteq \Omega^{\prime}$, and let $f^{\prime}=$ $\mathbb{E}\left[1_{X} \mid \varphi^{-1}(\mathcal{F})\right]$. Then there exists $f \in L^{1}(\Omega)$ so that $f^{\prime}=f^{\varphi}$. Since $F^{\varphi}$ is $\varphi^{-1}(\mathcal{F})$ measurable,

$$
\frac{\int_{X^{\prime}} F^{\varphi} d \mu^{\prime}}{\sqrt{\mu^{\prime}(X)}}=\frac{\int_{\Omega^{\prime}} f^{\prime} F^{\varphi} d \mu^{\prime}}{\sqrt{\int_{\Omega} f^{\prime} d \mu^{\prime}}}=\frac{\int_{\Omega} f F d \mu}{\sqrt{\int_{\Omega} f d \mu}} .
$$

Fix $\varepsilon>0$. Since $\Omega$ is $\sigma$-finite, there exists $\Omega_{0} \subseteq \Omega$ with $\mu\left(\Omega_{0}\right)<\infty$ and

$$
\frac{\int_{\Omega_{0}} f F d \mu}{\sqrt{\int_{\Omega_{0}} f d \mu}} \geq(1-\varepsilon) \frac{\int_{\Omega} f F d \mu}{\sqrt{\int_{\Omega} f d \mu}}
$$

By the previous lemma, there exists a measurable set $X \subseteq \Omega_{0}$ so that

$$
\frac{\int_{X} F d \mu}{\sqrt{\mu(X)}} \geq \frac{\int_{\Omega_{0}} f F d \mu}{\sqrt{\int_{\Omega_{0}} f d \mu}} \geq(1-\varepsilon) \frac{\int_{\Omega} f F d \mu}{\sqrt{\int_{\Omega} f d \mu}} .
$$

Since this holds for any $\varepsilon>0$, this proves that $\|F\|_{\otimes} \geq\left\|F^{\varphi}\right\|_{\otimes}$.

Finally, we show that $\|U\|_{\otimes}=\left\|U^{\varphi}\right\|_{\otimes}$. As before, for any measurable $X, Y \subseteq \Omega$ with $\mu(X), \mu(Y)<\infty$, since $\mu$ is the pushforward of $\mu^{\prime}$,

$$
\frac{\int_{\varphi^{-1}(X) \times \varphi^{-1}(Y)} U^{\varphi}\left(d \mu^{\prime}\right)^{2}}{\sqrt{\mu^{\prime}\left(\varphi^{-1}(X)\right)} \sqrt{\mu^{\prime}\left(\varphi^{-1}(Y)\right)}}=\frac{\int_{X \times Y} U(d \mu)^{2}}{\sqrt{\mu(X)} \sqrt{\mu(Y)}} .
$$

This shows that $\|U\|_{\otimes} \leq\left\|U^{\varphi}\right\|_{\otimes}$. For the other direction, let $X^{\prime}, Y^{\prime} \subseteq \Omega^{\prime}$ with finite measure. By the previous argument, there exist functions $f, g: \Omega \rightarrow[0,1]$ such that

$$
\frac{\int_{\Omega^{2}} f(x) U(x, y) g(y) d \mu(x) d \mu(y)}{\sqrt{\int_{\Omega} f d \mu \int_{\Omega} g d \mu}}=\frac{\int_{X^{\prime} \times Y^{\prime}} U^{\varphi}\left(d \mu^{\prime}\right)^{2}}{\sqrt{\mu^{\prime}\left(X^{\prime}\right) \mu^{\prime}\left(Y^{\prime}\right)}} .
$$

Fix $\varepsilon>0$. By the previous argument, there exists $X \subseteq \Omega$ such that

$$
\frac{\int_{X \times \Omega} U(x, y) g(y) d \mu(x) d \mu(y)}{\sqrt{\mu(X) \int_{\Omega} g d \mu}} \geq(1-\varepsilon) \frac{\int_{\Omega^{2}} f(x) U(x, y) g(y) d \mu(x) d \mu(y)}{\sqrt{\int_{\Omega} f d \mu \int_{\Omega} g d \mu}} .
$$

Then, applying it again, there exists $Y \subseteq \Omega$ such that

$$
\frac{\int_{X \times Y} U(x, y) d \mu(x) d \mu(y)}{\sqrt{\mu(X) \mu(Y)}} \geq(1-\varepsilon) \frac{\int_{X \times \Omega} U(x, y) g(y) d \mu(x) d \mu(y)}{\sqrt{\mu(X) \int_{\Omega} g}} .
$$

Combining these, we obtain

$$
\frac{\int_{X \times Y} U(x, y) d \mu(x) d \mu(y)}{\sqrt{\mu(X) \mu(Y)}} \geq(1-\varepsilon)^{2} \frac{\int_{X^{\prime} \times Y^{\prime}} U^{\varphi} d \mu^{\prime}}{\sqrt{\mu^{\prime}\left(X^{\prime}\right) \mu^{\prime}\left(Y^{\prime}\right)}} .
$$

Since this holds for any $\varepsilon>0$, we obtain that $\|U\|_{\bigotimes} \geq\left\|U^{\varphi}\right\|_{\bigotimes}$.

Next we establish a sequence of lemmas leading to the proof of Theorem 2.11, which states that $\delta_{2 \rightarrow 2}$ is a pseudometric. To state the first lemma, we define the vectors

$$
\begin{aligned}
\Delta_{2 \rightarrow 2}\left(\mathbb{W}_{1}, \mathbb{W}_{2}\right) & =\left(\left\|W_{1}-W_{2}\right\|_{2 \rightarrow 2},\left\|D_{\mathbb{W}_{1}}-D_{\mathbb{W}_{2}}\right\|_{2},\left|\rho\left(\mathbb{W}_{1}\right)-\rho\left(\mathbb{W}_{2}\right)\right|\right) \quad \text { and } \\
\Delta_{\bigotimes}\left(\mathbb{W}_{1}, \mathbb{W}_{2}\right) & =\left(\left\|W_{1}-W_{2}\right\|_{\bigotimes},\left\|D_{\mathbb{W}_{1}}-D_{\mathbb{W}_{2}}\right\|_{\bigotimes},\left|\rho\left(\mathbb{W}_{1}\right)-\rho\left(\mathbb{W}_{2}\right)\right|\right)
\end{aligned}
$$


where again we require the signed graphexes $\mathbb{W}_{1}$ and $\mathbb{W}_{2}$ to be in $L^{1} \cap L^{2}$. Note that each coordinate satisfies the triangle inequality. We will also use the following property.

Lemma 3.5. Let $\boldsymbol{\Omega}=(\Omega, \mathcal{F}, \mu)$ and $\boldsymbol{\Omega}^{\prime}=\left(\Omega^{\prime}, \mathcal{F}^{\prime}, \mu^{\prime}\right)$ be $\sigma$-finite measure spaces, let $\varphi: \Omega \rightarrow \Omega^{\prime}$ be a measure-preserving map, and let $\mathbb{W}_{1}, \mathbb{W}_{2}$ be signed graphexes in $L^{1} \cap L^{2}$. Then

$$
\Delta_{2 \rightarrow 2}\left(\mathbb{W}_{1}, \mathbb{W}_{2}\right)=\Delta_{2 \rightarrow 2}\left(\mathbb{W}_{1}^{\varphi}, \mathbb{W}_{2}^{\varphi}\right) \quad \text { and } \quad \Delta_{\otimes}\left(\mathbb{W}_{1}, \mathbb{W}_{2}\right)=\Delta_{\bigotimes}\left(\mathbb{W}_{1}^{\varphi}, \mathbb{W}_{2}^{\varphi}\right)
$$

Proof. Clearly $\rho\left(\mathbb{W}_{1}\right)=\rho\left(\mathbb{W}_{1}^{\varphi}\right)$ and $\rho\left(\mathbb{W}_{2}\right)=\rho\left(\mathbb{W}_{2}^{\varphi}\right)$, which means that their differences are equal too. We also have for every $x \in \Omega, D_{\mathbb{W}_{i}}(\varphi(x))=D_{\mathbb{W}_{i}^{\varphi}}(x)$, which implies that $\left\|D_{\mathbb{W}_{1}}-D_{\mathbb{W}_{2}}\right\|_{2}=\left\|D_{\mathbb{W}_{1}^{\varphi}}-D_{\mathbb{W}_{2}^{\varphi}}\right\|_{2}$. Therefore it remains to show that

as well as

$$
\left\|W_{1}-W_{2}\right\|_{2 \rightarrow 2}=\left\|W_{1}^{\varphi}-W_{2}^{\varphi}\right\|_{2 \rightarrow 2}
$$

$$
\left\|D_{\mathbb{W}_{1}}-D_{\mathbb{W}_{2}}\right\|_{\otimes}=\left\|D_{\mathbb{W}_{1}^{\varphi}}-D_{\mathbb{W}_{2}^{\varphi}}\right\|_{\otimes} \quad \text { and } \quad\left\|W_{1}-W_{2}\right\|_{\otimes}=\left\|W_{1}^{\varphi}-W_{2}^{\varphi}\right\|_{\otimes} .
$$

Note that $D_{\mathbb{W}_{1}^{\varphi}}-D_{\mathbb{W}_{2}^{\varphi}}=\left(D_{\mathbb{W}_{1}}-D_{\mathbb{W}_{2}}\right)^{\varphi}$ and $W_{1}^{\varphi}-W_{2}^{\varphi}=\left(W_{1}-W_{2}\right)^{\varphi}$, so by Proposition 3.4, we are done.

To prove the triangle inequality, we would like to take a coupling of $\Omega_{1}$ and $\Omega_{2}$ and a coupling of $\Omega_{2}$ and $\Omega_{3}$, and use them to obtain a coupling of $\Omega_{1}$ and $\Omega_{3}$. Unfortunately this cannot be done for general signed graphexes. We can, however do it if the signed graphexes involved are step graphexes. To define these, we first define a subspace partition of a measure space $\boldsymbol{\Omega}=(\Omega, \mathcal{F}, \mu)$ as a partition of a measurable subset $\Omega^{\prime} \subseteq \Omega$ into countably many measurable subsets. Such a subspace partition is called finite if it is a partition into finitely many sets of finite measure. A signed graphex $\mathbb{U}$ is then called a step graphex over the subspace partition $\mathcal{P}=\left(P_{1}, \ldots, P_{m}\right)$ if $\mathcal{P}$ is a finite subspace partition, dsupp $\mathbb{U} \subseteq P_{1} \cup P_{2} \cup \cdots \cup P_{m}$, and for all $x, x^{\prime} \in P_{i}$, $S(x)=S\left(x^{\prime}\right)$ and $W_{x}=W_{x^{\prime}}$, where, as before, $W_{x}$ is the function $y \mapsto W(x, y)$.

Remark 3.6. Given a signed step graphex $\mathbb{W}=(W, S, I, \boldsymbol{\Omega})$ over a finite subspace partition $\mathcal{P}=\left(P_{1}, P_{2}, \ldots, P_{m}\right)$, we can define another signed graphex $\mathbb{W}^{\prime}=$ $\left(W^{\prime}, S^{\prime}, I^{\prime}, \boldsymbol{\Omega}^{\prime}\right)$ with $\boldsymbol{\Omega}^{\prime}=\left(\Omega^{\prime}, \mathcal{F}^{\prime}, \mu^{\prime}\right)$, where $\Omega^{\prime}=[m], \mathcal{F}^{\prime}$ consists of all subsets, and the measure is defined by $\mu^{\prime}(\{i\})=\mu\left(P_{i}\right)$. Setting $I^{\prime}=I, S^{\prime}(i)=S(x)$ for any $x \in P_{i}$ (they are all equal), and $W^{\prime}(i, j)=W(x, y)$ for $x \in P_{i}, y \in P_{j}$ (again the choice of $x$ and $y$ does not matter), we obtain that $\mathbb{W}=\left(\mathbb{W}^{\prime}\right)^{\varphi}$ where $\varphi: \Omega \rightarrow \Omega^{\prime}$ is the map with $\varphi(x)=i$ for $x \in P_{i}$. In particular, by Remark 2.16, the distance between $\mathbb{W}$ and $\mathbb{W}^{\prime}$ is zero (for any of the distance notions). Suppose now that $\mathbb{W}^{\prime \prime}=\left(W^{\prime \prime}, S^{\prime \prime}, I^{\prime \prime}, \boldsymbol{\Omega}^{\prime \prime}\right)$ with $\boldsymbol{\Omega}^{\prime \prime}=\left(\Omega^{\prime \prime}, \mathcal{F}^{\prime \prime}, \mu^{\prime \prime}\right)$ is another signed step graphex over a finite subspace partition $\mathcal{Q}=\left\{Q_{1}, Q_{2}, \ldots, Q_{m}\right\}$, with $I^{\prime \prime}=I, \mu^{\prime \prime}\left(Q_{i}\right)=\mu\left(P_{i}\right)$, and $S^{\prime \prime}\left(x^{\prime \prime}\right)=S(x), W^{\prime \prime}\left(x^{\prime \prime}, y^{\prime \prime}\right)=W(x, y)$ for $x \in P_{i}, x^{\prime \prime} \in Q_{i}, y \in P_{j}, y^{\prime \prime} \in Q_{j}$. Then $\boldsymbol{\Omega}^{\prime \prime}$ can also be mapped to $\boldsymbol{\Omega}^{\prime}$ so that $\mathbb{W}^{\prime \prime}$ is the pullback of $\mathbb{W}^{\prime}$ (by mapping $Q_{i}$ to $i$ ). This implies that the distance of both $\mathbb{W}$ and $\mathbb{W}^{\prime \prime}$ from $\mathbb{W}^{\prime}$ is 0 , which (by the still to be proven triangle inequality) implies that their distance from each other is 0 (again for any of the notions of distance).

Returning to the proof of the triangle inequality, we will in fact consider signed graphexes that are countable step graphexes, i.e., the number of "steps" is countable, and each step has finite measure. First, however, we need the following technical lemma: 
Lemma 3.7. Let $\mathbb{W}_{1}=\left(W_{1}, S_{1}, I_{1}, \boldsymbol{\Omega}\right)$ and $\mathbb{W}_{2}=\left(W_{2}, S_{2}, I_{2}, \boldsymbol{\Omega}\right)$ be signed graphexes in $L^{1} \cap L^{2}$. Assume that both are countable step graphexes on $\Omega=(\Omega, \mathcal{F}, \mu)$ with common refinement $\mathcal{P}=\left\{P_{1}, P_{2}, \ldots, P_{m}, \ldots\right\}$, suppose $\mu^{\prime}$ is another measure on $\Omega$ with $\mu\left(P_{i}\right)=\mu^{\prime}\left(P_{i}\right)$, and let $\boldsymbol{\Omega}^{\prime}=\left(\Omega, \mathcal{F}, \mu^{\prime}\right)$ and $\mathbb{W}_{i}^{\prime}=\left(W_{i}, S_{i}, I_{i}, \boldsymbol{\Omega}^{\prime}\right)$. Then

$$
\Delta_{2 \rightarrow 2}\left(\mathbb{W}_{1}, \mathbb{W}_{2}\right)=\Delta_{2 \rightarrow 2}\left(\mathbb{W}_{1}^{\prime}, \mathbb{W}_{2}^{\prime}\right) \quad \text { and } \quad \Delta_{\bigotimes}\left(\mathbb{W}_{1}, \mathbb{W}_{2}\right)=\Delta_{\bigotimes}\left(\mathbb{W}_{1}^{\prime}, \mathbb{W}_{2}^{\prime}\right) \text {. }
$$

Proof. Let $\boldsymbol{\Omega}_{\mathcal{P}}=\left(\Omega_{\mathcal{P}}, \mathcal{F}_{\mathcal{P}}, \mu_{\mathcal{P}}\right)$ where $\Omega_{\mathcal{P}}=\left\{x_{1}, x_{2}, \ldots, x_{m}, \ldots\right\}, \mathcal{F}_{\mathcal{P}}$ is the set of all subsets of $\Omega_{\mathcal{P}}$, and $\mu_{\mathcal{P}}\left(x_{i}\right)=\mu\left(P_{i}\right)$. Let $\varphi, \varphi^{\prime}: \Omega \rightarrow \Omega_{\mathcal{P}}$ with $\varphi(x)=\varphi^{\prime}(x)=x_{i}$ for $x \in P_{i}$. Then $\varphi: \boldsymbol{\Omega} \rightarrow \boldsymbol{\Omega}_{\mathcal{P}}$ and $\varphi^{\prime}: \boldsymbol{\Omega}^{\prime} \rightarrow \boldsymbol{\Omega}_{\mathcal{P}}$ are both measure preserving (these are the same function on $\Omega$ but as maps between measure spaces are different). Define $\mathbb{W}_{i, \mathcal{P}}=\left(W_{i, \mathcal{P}}, S_{i, \mathcal{P}}, I_{i}\right)$ with $S_{i, \mathcal{P}}\left(x_{j}\right)=S_{i}(x)$ for any $x \in P_{j}$ (they are all equal), and $W_{i, \mathcal{P}}\left(x_{j}, x_{k}\right)=W(x, y)$ for $x \in P_{j}, y \in P_{k}$ (again, they are all equal). Then $\mathbb{W}_{i, \mathcal{P}}^{\varphi}=\mathbb{W}_{i}$ and $\mathbb{W}_{i, \mathcal{P}}^{\varphi^{\prime}}=\mathbb{W}_{i}^{\prime}$. Therefore, by Lemma 3.5, we have

$$
\Delta_{2 \rightarrow 2}\left(\mathbb{W}_{1}, \mathbb{W}_{2}\right)=\Delta_{2 \rightarrow 2}\left(\mathbb{W}_{1, \mathcal{P}}, \mathbb{W}_{2, \mathcal{P}}\right)=\Delta_{2 \rightarrow 2}\left(\mathbb{W}_{1}^{\prime}, \mathbb{W}_{2}^{\prime}\right)
$$

and similarly for $\Delta_{\bigotimes}$.

To state the next lemma, we use the symbol $\pi_{i j, k}$ to denote the coordinate projection from a product space $\Omega_{i} \times \Omega_{j}$ to $\Omega_{k}$, where $k=i$ or $k=j$.

Lemma 3.8. Let $\mathbb{W}_{i}=\left(W_{i}, S_{i}, I_{i}, \boldsymbol{\Omega}_{i}\right)$, for $i=1,2,3$, be countable step graphexes in $L^{1} \cap L^{2}$. Let $\mu_{12}$ be a coupling measure on $\Omega_{1} \times \Omega_{2}$, and $\mu_{23}$ be a coupling measure on $\Omega_{2} \times \Omega_{3}$. Then there exists a coupling measure $\mu_{13}$ on $\Omega_{1}$ and $\Omega_{3}$ such that

$$
\begin{aligned}
\Delta_{2 \rightarrow 2}( & \left.\mathbb{W}_{1}^{\pi_{13,1}, \mu_{13}}, \mathbb{W}_{3}^{\pi_{13,3}, \mu_{13}}\right) \\
& \leq \Delta_{2 \rightarrow 2}\left(\mathbb{W}_{1}^{\pi_{12,1}, \mu_{12}}, \mathbb{W}_{3}^{\pi_{12,2}, \mu_{12}}\right)+\Delta_{2 \rightarrow 2}\left(\mathbb{W}_{1}^{\pi_{23,2}, \mu_{23}}, \mathbb{W}_{3}^{\pi_{23,3}, \mu_{23}}\right)
\end{aligned}
$$

and

$$
\begin{aligned}
& \Delta_{\bigotimes}\left(\mathbb{W}_{1}^{\pi_{13,1}, \mu_{13}}, \mathbb{W}_{3}^{\pi_{13,3}, \mu_{13}}\right) \\
& \quad \leq \Delta_{\bigotimes}\left(\mathbb{W}_{1}^{\pi_{12,1}, \mu_{12}}, \mathbb{W}_{3}^{\pi_{12,2}, \mu_{12}}\right)+\Delta_{\bigotimes}\left(\mathbb{W}_{1}^{\pi_{23,2}, \mu_{23}}, \mathbb{W}_{3}^{\pi_{23,3}, \mu_{23}}\right),
\end{aligned}
$$

where the inequalities hold coordinate-wise.

Proof. Let the steps of $\mathbb{W}_{1}$ be $A_{1}, A_{2}, \ldots$, the steps of $\mathbb{W}_{2}$ be $B_{1}, B_{2}, \ldots$, and the steps of $\mathbb{W}_{3}$ be $C_{1}, C_{2}, \ldots$ Without loss of generality, we may assume that each $\mu_{1}\left(A_{p}\right)>0$, each $\mu_{2}\left(B_{q}\right)>0$, and each $\mu_{3}\left(C_{r}\right)>0$. First, take the measure $\mu_{123}$ on $\Omega_{1} \times \Omega_{2} \times \Omega_{3}$ where

$$
\mu_{123}(E)=\sum_{p, q, r} \frac{\mu_{12}\left(A_{p} \times B_{q}\right) \mu_{23}\left(B_{q} \times C_{r}\right)}{\mu_{1}\left(A_{p}\right) \mu_{2}\left(B_{q}\right)^{2} \mu_{3}\left(C_{r}\right)}\left(\mu_{1} \times \mu_{2} \times \mu_{3}\right)\left(E \cap A_{p} \times B_{q} \times C_{r}\right) .
$$

Then

$$
\begin{aligned}
\mu_{123}\left(A_{p_{0}} \times B_{q_{0}} \times \Omega_{3}\right)=\sum_{p, q, r} \frac{\mu_{12}\left(A_{p} \times B_{q}\right) \mu_{23}\left(B_{q} \times C_{r}\right)}{\mu_{1}\left(A_{p}\right) \mu_{2}\left(B_{q}\right)^{2} \mu_{3}\left(C_{r}\right)} \\
\cdot\left(\mu_{1} \times \mu_{2} \times \mu_{3}\right)\left(A_{p_{0}} \times B_{q_{0}} \times \Omega_{3} \cap A_{p} \times B_{q} \times C_{r}\right) \\
=\mu_{12}\left(A_{p_{0}} \times B_{q_{0}}\right) \sum_{r} \frac{\mu_{23}\left(B_{q_{0}} \times C_{r}\right)}{\mu_{1}\left(A_{p_{0}}\right) \mu_{2}\left(B_{q_{0}}\right)^{2} \mu_{3}\left(C_{r}\right)} \mu_{1}\left(A_{p_{0}}\right) \mu_{2}\left(B_{q_{0}}\right) \mu_{3}\left(C_{r}\right) \\
=\mu_{12}\left(A_{p_{0}} \times B_{q_{0}}\right) \sum_{r} \frac{\mu_{23}\left(B_{q_{0}} \times C_{r}\right)}{\mu_{2}\left(B_{q_{0}}\right)}=\mu_{12}\left(A_{p_{0}} \times B_{q_{0}}\right) .
\end{aligned}
$$


In other words, if $\pi_{123,12}$ is the projection from $\Omega_{1} \times \Omega_{2} \times \Omega_{3}$ to $\Omega_{1} \times \Omega_{2}$ and $\mu_{12}^{\prime}=\mu_{123}^{\pi_{123,12}}$, then $\mu_{12^{\prime}}\left(A_{p} \times B_{q}\right)=\mu_{12}\left(A_{p} \times B_{q}\right)$. Analogously, if $\mu_{23}^{\prime}=\mu_{123}^{\pi_{123,23}}$, then $\mu_{23^{\prime}}\left(A_{p} \times B_{q}\right)=\mu_{23}\left(A_{p} \times B_{q}\right)$. Furthermore, for any $F \subseteq \Omega_{1}$,

$$
\begin{aligned}
& \mu_{123}\left(F \times \Omega_{2} \times \Omega_{3}\right) \\
& =\sum_{p, q, r} \frac{\mu_{12}\left(A_{p} \times B_{q}\right) \mu_{23}\left(B_{q} \times C_{r}\right)}{\mu_{1}\left(A_{p}\right) \mu_{2}\left(B_{q}\right)^{2} \mu_{3}\left(C_{r}\right)}\left(\mu_{1} \times \mu_{2} \times \mu_{3}\right)\left(F \times \Omega_{2} \times \Omega_{3} \cap A_{p} \times B_{q} \times C_{r}\right) \\
& =\sum_{p, q, r} \frac{\mu_{12}\left(A_{p} \times B_{q}\right) \mu_{23}\left(B_{q} \times C_{r}\right)}{\mu_{1}\left(A_{p}\right) \mu_{2}\left(B_{q}\right)^{2} \mu_{3}\left(C_{r}\right)} \mu_{1}\left(F \cap A_{p}\right) \mu_{2}\left(B_{q}\right) \mu_{3}\left(C_{r}\right) \\
& =\sum_{p, q, r} \frac{\mu_{12}\left(A_{p} \times B_{q}\right) \mu_{23}\left(B_{q} \times C_{r}\right)}{\mu_{1}\left(A_{p}\right) \mu_{2}\left(B_{q}\right)} \mu_{1}\left(F \cap A_{p}\right)=\sum_{p, q} \frac{\mu_{12}\left(A_{p} \times B_{q}\right)}{\mu_{1}\left(A_{p}\right)} \mu_{1}\left(F \cap A_{p}\right) \\
& =\sum_{p} \frac{\mu_{12}\left(A_{p} \times \Omega_{2}\right)}{\mu_{1}\left(A_{p}\right)} \mu_{1}\left(F \cap A_{p}\right)=\sum_{p} \mu_{1}\left(F \cap A_{p}\right)=\mu_{1}(F) .
\end{aligned}
$$

Analogously, for any $G \subseteq \Omega_{3}, \mu_{123}\left(\Omega_{1} \times \Omega_{2} \times G\right)=\mu_{3}(G)$. Therefore, $\mu_{13}=\mu_{123}^{\pi_{123,13}}$ is a coupling measure on $\Omega_{1} \times \Omega_{3}$ of $\mu_{1}$ and $\mu_{3}$. By Lemma 3.5 and the triangle inequality for the coordinates of $\Delta_{2 \rightarrow 2}$, we then have

$$
\begin{aligned}
\Delta_{2 \rightarrow 2} & \left(\mathbb{W}_{1}^{\pi_{13,1}, \mu_{13}}, \mathbb{W}_{3}^{\pi_{13,3}, \mu_{13}}\right) \\
& =\Delta_{2 \rightarrow 2}\left(\mathbb{W}_{1}^{\pi_{123,1}, \mu_{123}}, \mathbb{W}_{3}^{\pi_{123,3}, \mu_{123}}\right) \\
& \leq \Delta_{2 \rightarrow 2}\left(\mathbb{W}_{1}^{\pi_{123,1}, \mu_{123}}, \mathbb{W}_{2}^{\pi_{123,2}, \mu_{123}}\right)+\Delta_{2 \rightarrow 2}\left(\mathbb{W}_{2}^{\pi_{123,2}, \mu_{123}}, \mathbb{W}_{3}^{\pi_{123,3}, \mu_{123}}\right) \\
& =\Delta_{2 \rightarrow 2}\left(\mathbb{W}_{1}^{\pi_{12,1}, \mu_{12}^{\prime}}, \mathbb{W}_{2}^{\pi_{12,2}, \mu_{12}^{\prime}}\right)+\Delta_{2 \rightarrow 2}\left(\mathbb{W}_{2}^{\pi_{23,2}, \mu_{23}^{\prime}}, \mathbb{W}_{3}^{\pi_{23,3}, \mu_{23}^{\prime}}\right) \\
& =\Delta_{2 \rightarrow 2}\left(\mathbb{W}_{1}^{\pi_{12,1}, \mu_{12}}, \mathbb{W}_{2}^{\pi_{12,2}, \mu_{12}}\right)+\Delta_{2 \rightarrow 2}\left(\mathbb{W}_{2}^{\pi_{23,2}, \mu_{23}}, \mathbb{W}_{3}^{\pi_{23,3}, \mu_{23}}\right) .
\end{aligned}
$$

The proof for $\Delta_{\otimes}$ is the same.

We are now ready to prove that $\widetilde{\delta}_{2 \rightarrow 2}$ and the distance $\widetilde{\delta}_{\square}$ (obtained by replacing $d_{2 \rightarrow 2}$ in $(2.3)$ with $\left.d_{\bigotimes}\right)$ obey the triangle inequality.

Lemma 3.9. Suppose that $\mathbb{W}_{i}=\left(W_{i}, S_{i}, I_{i}, \boldsymbol{\Omega}_{i}\right)$ with $\boldsymbol{\Omega}_{i}=\left(\Omega_{i}, \mathcal{F}_{i}, \mu_{i}\right)$, for $i=$ $1,2,3$, are signed graphexes in $L^{1} \cap L^{2}$, and assume that $\mu_{1}\left(\Omega_{1}\right)=\mu_{2}\left(\Omega_{2}\right)=\mu_{3}\left(\Omega_{3}\right)$. Then

$$
\widetilde{\delta}_{2 \rightarrow 2}\left(\mathbb{W}_{1}, \mathbb{W}_{3}\right) \leq \widetilde{\delta}_{2 \rightarrow 2}\left(\mathbb{W}_{1}, \mathbb{W}_{2}\right)+\widetilde{\delta}_{2 \rightarrow 2}\left(\mathbb{W}_{2}, \mathbb{W}_{3}\right)
$$

and

$$
\widetilde{\delta}_{\bigotimes}\left(\mathbb{W}_{1}, \mathbb{W}_{3}\right) \leq \widetilde{\delta}_{\bigotimes}\left(\mathbb{W}_{1}, \mathbb{W}_{2}\right)+\widetilde{\delta}_{凶}\left(\mathbb{W}_{2}, \mathbb{W}_{3}\right) .
$$

Proof. We first claim that it is enough to prove that for any coupling measure $\mu_{12}$ on $\Omega_{1} \times \Omega_{2}$, any coupling measure $\mu_{23}$ on $\Omega_{2} \times \Omega_{3}$ and any $\varepsilon>0$, there exists a coupling measure $\mu_{13}$ on $\Omega_{1} \times \Omega_{3}$, such that

$$
\begin{aligned}
& \Delta_{2 \rightarrow 2}\left(\mathbb{W}_{1}^{\pi_{13,1}, \mu_{13}}, \mathbb{W}_{3}^{\pi_{13,3}, \mu_{13}}\right) \\
& \quad \leq \Delta_{2 \rightarrow 2}\left(\mathbb{W}_{1}^{\pi_{12,1}, \mu_{12}}, \mathbb{W}_{2}^{\pi_{12,2}, \mu_{12}}\right)+\Delta_{2 \rightarrow 2}\left(\mathbb{W}_{2}^{\pi_{23,2}, \mu_{23}}, \mathbb{W}_{3}^{\pi_{23,3}, \mu_{23}}\right)+(\varepsilon, \varepsilon, \varepsilon)
\end{aligned}
$$

and

$$
\begin{aligned}
& \Delta_{\bigotimes}\left(\mathbb{W}_{1}^{\pi_{13,1}, \mu_{13}}, \mathbb{W}_{3}^{\pi_{13,3}, \mu_{13}}\right) \\
& \quad \leq \Delta_{\bigotimes}\left(\mathbb{W}_{1}^{\pi_{12,1}, \mu_{12}}, \mathbb{W}_{2}^{\pi_{12,2}, \mu_{12}}\right)+\Delta_{\bigotimes}\left(\mathbb{W}_{2}^{\pi_{23,2}, \mu_{23}}, \mathbb{W}_{3}^{\pi_{23,3}, \mu_{23}}\right)+(\varepsilon, \varepsilon, \varepsilon)
\end{aligned}
$$


Indeed, given that $\varepsilon>0$ is arbitrary, (3.5) clearly implies the triangle inequality for $\widetilde{\delta}_{\square}$. To see that (3.4) implies the triangle inequality for $\widetilde{\delta}_{2 \rightarrow 2}$, observe that $(x+y)^{1 / k} \leq x^{1 / k}+y^{1 / k}$ whenever $k \geq 1$.

Next, we claim that for any $\varepsilon>0$, any $\mathbb{W}=(W, S, I, \boldsymbol{\Omega})$ in $L^{1} \cap L^{2}$ can be approximated by a signed step graphex $\mathbb{W}^{\prime}$ such that

$$
\Delta_{\Downarrow}\left(\mathbb{W}, \mathbb{W}^{\prime}\right) \leq \Delta_{2 \rightarrow 2}\left(\mathbb{W}, \mathbb{W}^{\prime}\right) \leq(\varepsilon, \varepsilon, \varepsilon) .
$$

Indeed, let $\boldsymbol{\Omega}=(\Omega, \mathcal{F}, \mu)$, let $\Omega_{1} \subseteq \Omega_{2} \subseteq \cdots \subseteq \Omega$ be such that $\mu\left(\Omega_{n}\right)<\infty$ and $\Omega=\bigcup_{n} \Omega_{n}$, and let $\mathbb{W}_{n}=\left(W_{n}, S_{n}, I, \boldsymbol{\Omega}\right)$, where $W_{n}=W 1_{\Omega_{n} \times \Omega_{n}}$ and $S_{n}=S 1_{\Omega_{n}}$. Using the dominated convergence theorem and the assumption that $\mathbb{W}$ is in $L^{1} \cap L^{2}$, we then have that

$$
\begin{gathered}
\left|\rho(\mathbb{W})-\rho\left(\mathbb{W}_{n}\right)\right| \leq\left\|W-W_{n}\right\|_{1}=\left\||W|\left(1-1_{\Omega_{n} \times \Omega_{n}}\right)\right\|_{1} \rightarrow 0, \\
\left\|W-W_{n}\right\|_{2 \rightarrow 2} \leq\left\|W-W_{n}\right\|_{2}=\left\|W\left(1-1_{\Omega_{n} \times \Omega_{n}}\right)\right\|_{2} \rightarrow 0,
\end{gathered}
$$

and

$$
\left\|S-S_{n}\right\|_{2}=\left\|S\left(1-1_{\Omega_{n}}\right)\right\|_{2} \rightarrow 0
$$

as $n \rightarrow \infty$. Next, defining $\chi_{n}$ by $\chi_{n}(x, y, z)=\left(1-1_{\Omega_{n} \times \Omega_{n}}(x, y)\right)\left(1-1_{\Omega_{n} \times \Omega_{n}}(y, z)\right)$, we bound

$$
\left\|D_{W}-D_{W_{n}}\right\|_{2} \leq \sqrt{\int|W(x, y)||W(y, z)| \chi_{n}(x, y, z) d \mu(x) d \mu(y) d \mu(z)} .
$$

Since $\chi_{n}$ goes to zero pointwise and $D_{|W|}$ is in $L^{2}$, the right side again goes to zero by the dominated convergence theorem. Therefore,

$$
\left\|D_{\mathbb{W}}-D_{\mathbb{W}_{n}}\right\|_{2} \leq\left\|D_{W}-D_{W_{n}}\right\|_{2}+\left\|S-S_{n}\right\|_{2} \rightarrow 0 .
$$

This shows that for $n$ large enough $\Delta_{2 \rightarrow 2}\left(\mathbb{W}, \mathbb{W}_{n}\right) \leq \varepsilon / 4$.

Fixing $n$ such that this holds, we now define $W^{(k)}=W_{n} 1_{\left|W_{n}\right| \leq k}$ and $S^{(k)}=$ $S_{n} 1_{\left|S_{n}\right| \leq k}$. Another application of the dominated convergence theorem then shows that for $k$ large enough, $\Delta_{2 \rightarrow 2}\left(\mathbb{W}^{(k)}, \mathbb{W}_{n}\right) \leq \varepsilon / 4$, giving us a graphon $\mathbb{W}^{\prime \prime}=$ $\left(W^{\prime \prime}, S^{\prime \prime}, I, \boldsymbol{\Omega}\right)$ such that the degree support of $\mathbb{W}^{\prime \prime}$ has finite measure, both $W^{\prime \prime}$ and $D_{\mathbb{W}}$ are bounded, and $\Delta_{2 \rightarrow 2}\left(\mathbb{W}^{\prime \prime}, \mathbb{W}\right) \leq \varepsilon / 2$. But such a graphex can be approximated to arbitrary precision by a step graphex with finitely many steps, proving the claim for $\Delta_{2 \rightarrow 2}$. Since on two variable functions, $\|\cdot\|_{\otimes}$ is bounded by $\|\cdot\|_{2 \rightarrow 2}$, and on functions of one variable it is bounded by $\|\cdot\|_{2}$, the claim for $\Delta_{\bigotimes}$ follows as well.

Fix $\varepsilon>0$, and let $\mathbb{W}_{1}^{\prime}, \mathbb{W}_{2}^{\prime}, \mathbb{W}_{3}^{\prime}$ be approximations of $\mathbb{W}_{1}, \mathbb{W}_{2}, \mathbb{W}_{3}$ by signed step graphexes such that for $k=1,2,3$,

$$
\Delta_{2 \rightarrow 2}\left(\mathbb{W}_{k}, \mathbb{W}_{k}^{\prime}\right) \leq(\varepsilon / 6, \varepsilon / 6, \varepsilon / 6) .
$$

If $\mu_{i j}$ is a coupling measure on $\Omega_{i} \times \Omega_{j}$ and $\pi_{i j, k}$ is the projection onto $\Omega_{k}, k=i$ or $j$, then $\pi_{i j, k}$ is measure preserving. Therefore, by Lemma 3.5,

$$
\Delta_{2 \rightarrow 2}\left(\left(\mathbb{W}_{k}\right)^{\pi_{i j, k}, \mu_{i j}},\left(\mathbb{W}_{k}^{\prime}\right)^{\pi_{i j, k}, \mu_{i j}}\right) \leq(\varepsilon / 6, \varepsilon / 6, \varepsilon / 6) .
$$


Combined with Lemma 3.8, we conclude that there exists a coupling measure $\mu_{13}$ on $\Omega_{1} \times \Omega_{3}$ such that

$$
\begin{gathered}
\Delta_{2 \rightarrow 2}\left(\mathbb{W}_{1}^{\pi_{13,1}, \mu_{13}}, \mathbb{W}_{3}^{\pi_{13,3}, \mu_{13}}\right) \leq \Delta_{2 \rightarrow 2}\left(\left(\mathbb{W}_{1}^{\prime}\right)^{\pi_{13,1}, \mu_{13}},\left(\mathbb{W}_{3}^{\prime}\right)^{\pi_{13,3}, \mu_{13}}\right)+(\varepsilon / 3, \varepsilon / 3, \varepsilon / 3) \\
\leq \Delta_{2 \rightarrow 2}\left(\left(\mathbb{W}_{1}^{\prime}\right)^{\pi_{12,1}, \mu_{12}},\left(\mathbb{W}_{2}^{\prime}\right)^{\pi_{12,2}, \mu_{12}}\right)+\Delta_{2 \rightarrow 2}\left(\left(\mathbb{W}_{2}^{\prime}\right)^{\pi_{23,2}, \mu_{23}},\left(\mathbb{W}_{3}^{\prime}\right)^{\pi_{23,3}, \mu_{23}}\right) \\
\quad+(\varepsilon / 3, \varepsilon / 3, \varepsilon / 3) \\
\leq \Delta_{2 \rightarrow 2}\left(\mathbb{W}_{1}^{\pi_{12,1}, \mu_{12}}, \mathbb{W}_{2}^{\pi_{12,2}, \mu_{12}}\right)+\Delta_{2 \rightarrow 2}\left(\mathbb{W}_{2}^{\pi_{23,2}, \mu_{23}}, \mathbb{W}_{3}^{\pi_{23,3}, \mu_{23}}\right)+(\varepsilon, \varepsilon, \varepsilon)
\end{gathered}
$$

proving (3.4) and hence the first statement of the lemma. The proof of (3.5) and the second statement follows in the same way.

The proof of Theorems 2.11 and 3.1 will be an easy corollary of Lemma 3.9 and the following extension lemma.

Lemma 3.10. Let $\mathbb{W}=(W, S, I, \boldsymbol{\Omega})$ be a signed graphex in $L^{1} \cap L^{2}$, with possibly unbounded graphon parts, and let $\boldsymbol{\Omega}=(\Omega, \mathcal{F}, \mu)$.

(1) If $\mathbb{W}^{\prime}$ and $\mathbb{W}^{\prime \prime}$ are trivial extensions of $\mathbb{W}$ by $\sigma$-finite spaces of infinite measure, then

$$
\widetilde{\delta}_{\square}\left(\mathbb{W}^{\prime}, \mathbb{W}^{\prime \prime}\right)=\widetilde{\delta}_{2 \rightarrow 2}\left(\mathbb{W}^{\prime}, \mathbb{W}^{\prime \prime}\right)=0 .
$$

(2) If $\mu(\Omega)=\infty$ and $\widetilde{\mathbb{W}}=(\widetilde{W}, \widetilde{S}, I, \widetilde{\boldsymbol{\Omega}})$ is obtained from $\mathbb{W}$ by appending an arbitrary $\sigma$-finite space of infinite measure, then

$$
\widetilde{\delta}_{2 \rightarrow 2}(\mathbb{W}, \widetilde{\mathbb{W}})=\widetilde{\delta}_{凶}(\mathbb{W}, \widetilde{\mathbb{W}})=0 .
$$

Proof. To prove the first statement, let $\Omega^{\prime}=\left(\Omega^{\prime}, \mathcal{F}^{\prime}, \mu^{\prime}\right)$ and $\boldsymbol{\Omega}^{\prime \prime}=\left(\Omega^{\prime \prime}, \mathcal{F}^{\prime \prime}, \mu^{\prime \prime}\right)$ be the spaces $\Omega$ has been extended by. Let $\widehat{\mu}$ be the measure on $\Omega \times \Omega$ which couples $\mu$ to itself along the diagonal, choose an arbitrary coupling $\widetilde{\mu}$ of $\mu^{\prime}$ and $\mu^{\prime \prime}$, and let $\widehat{\mu}^{\prime}$ be the measure on $\left(\Omega \cup \Omega^{\prime}\right) \times\left(\Omega \cup \Omega^{\prime \prime}\right)$ defined by

$$
\widehat{\mu}^{\prime}(A)=\widehat{\mu}(A \cap(\Omega \times \Omega))+\widetilde{\mu}\left(A \cap\left(\Omega^{\prime} \times \Omega^{\prime \prime}\right)\right) .
$$

Using the fact that $\widehat{\mu}^{\prime}\left(\Omega \times \Omega^{\prime \prime}\right)=\widehat{\mu}^{\prime}\left(\Omega^{\prime} \times \Omega\right)=0$, it is easy to see that

$$
\widetilde{\delta}_{2 \rightarrow 2}\left(\mathbb{W}^{\prime}, \mathbb{W}^{\prime \prime}\right) \leq d_{2 \rightarrow 2}\left(\left(\mathbb{W}^{\prime}\right)^{\pi_{1}, \widehat{\mu}^{\prime}},\left(\mathbb{W}^{\prime \prime}\right)^{\pi_{2}, \widehat{\mu}^{\prime}}\right)=d_{2 \rightarrow 2}\left(\mathbb{W}^{\pi_{1}, \widehat{\mu}}, \mathbb{W}^{\pi_{2}, \widehat{\mu}}\right)=0 .
$$

This proves the first statement for the metric $\widetilde{\delta}_{2 \rightarrow 2}$. The proof for the metric $\widetilde{\delta}_{\square}$ is identical.

To prove, the second statement, let $\widetilde{\boldsymbol{\Omega}}=(\widetilde{\Omega}, \widetilde{\mathcal{F}}, \widetilde{\mu})$. Since $\boldsymbol{\Omega}$ is $\sigma$-finite, we can find a sequence of measurable subsets $\Omega_{n} \subseteq \Omega$ such that $\Omega=\bigcup \Omega_{n}$ and each $\Omega_{n}$ has finite measure. Replacing $\Omega_{n}$ by $\Omega_{1} \cup \cdots \cup \Omega_{n}$, we may further assume that $\Omega_{n}$ is an increasing sequence of sets. Let $W_{n}$ be equal to $W$ on $\Omega_{n} \times \Omega_{n}$ and 0 everywhere else, and let $S_{n}=S$ on $\Omega_{n}$ and 0 outside of $\Omega_{n}$. Let $\mathbb{W}_{n}$ be the corresponding graphex on $\boldsymbol{\Omega}$ (with the same value $I$ ), and $\widetilde{\mathbb{W}}_{n}$ be its trivial extension to $\widetilde{\boldsymbol{\Omega}}$. By monotone convergence, $W_{n} \rightarrow W$ in both $L^{1}$ and $L^{2}$, and $S_{n} \rightarrow S$ in $L^{1}$ and $L^{2}$, implying that $\widetilde{\delta}_{2 \rightarrow 2}\left(\mathbb{W}_{n}, \mathbb{W}\right) \leq d_{2 \rightarrow 2}\left(\mathbb{W}_{n}, \mathbb{W}\right) \rightarrow 0$. For the same reason, $\widetilde{\delta}_{2 \rightarrow 2}\left(\widetilde{\mathbb{W}}_{n}, \widetilde{\mathbb{W}}\right) \rightarrow 0$. But since $\widetilde{\mathbb{W}}_{n}$ and $\mathbb{W}_{n}$ can both be obtained from the restriction of $\mathbb{W}_{n}$ to $\Omega_{n}$ by appending a space of infinite total measure, we have $\widetilde{\delta}_{2 \rightarrow 2}\left(\mathbb{W}_{n}, \widetilde{\mathbb{W}}_{n}\right)=\widetilde{\delta}_{2 \rightarrow 2}\left(\mathbb{W}_{n}, \mathbb{W}_{n}\right)=0$ by the first statement of the lemma. Using the triangle inequality for $\widetilde{\delta}_{2 \rightarrow 2}$, this proves the second statement for the distance $\widetilde{\delta}_{2 \rightarrow 2}$. The proof for the metric $\widetilde{\delta}_{\square}$ follows from the fact that the jumble norm is 
bounded by the kernel norm, which in turn implies that $d_{凶}\left(\mathbb{W}_{n}, \mathbb{W}\right) \rightarrow 0$ whenever $d_{2 \rightarrow 2}\left(\mathbb{W}_{n}, \mathbb{W}\right) \rightarrow 0$.

We are now ready to prove Theorems 2.11 and 3.1.

Proof of Theorems 2.11 and 3.1. The first statement of Lemma 3.10 implies that if $\mathbb{W}_{1}^{\prime}$ and $\mathbb{W}_{2}^{\prime}$ are trivial extensions of $\mathbb{W}_{1}$ and $\mathbb{W}_{2}$ obtained by appending two $\sigma$-finite spaces of infinite measure, then $\widetilde{\delta}_{2 \rightarrow 2}\left(\mathbb{W}_{1}^{\prime}, \mathbb{W}_{2}^{\prime}\right)$ and $\widetilde{\delta}_{\otimes}\left(\mathbb{W}_{1}^{\prime}, \mathbb{W}_{2}^{\prime}\right)$ do not depend on the choice of these extensions, and the second (combined with the triangle inequality) allows us to conclude that this remains true for extensions to spaces of infinite measure, which completes the proof of the first statements of the two theorems.

Since clearly $\delta_{2 \rightarrow 2}$ is symmetric and $\delta_{2 \rightarrow 2}(\mathbb{W}, \mathbb{W})=0$ for all integrable graphexes, all that remains to be proved is the triangle inequality for $\delta_{2 \rightarrow 2}$, which follows from the (already established) triangle inequality for $\widetilde{\delta}_{2 \rightarrow 2}$. The same holds for $\widetilde{\delta}_{\square}$.

The following example shows that the extension to infinite spaces in the definition of $\delta_{2 \rightarrow 2}$ is really needed.

Example 3.11. Let $\mathbb{W}_{1}=\left(W_{1}, 0,0, \boldsymbol{\Omega}_{1}\right)$ where $\boldsymbol{\Omega}_{1}$ consists of just two atoms $a$ and $b$, with weight $p$ and $1-p$, where $0<p<1 / 2$, and $W_{1}(a, a)=W_{1}(b, b)=0$, $W_{1}(a, b)=W_{1}(b, a)=1$. Furthermore, let $\mathbb{W}_{2}=\left(W_{2}, 0,0, \boldsymbol{\Omega}_{2}\right)$ where $\boldsymbol{\Omega}_{2}$ consists of just one atom $c$ with weight 1 , and $W_{2}$ is the constant $a=\sqrt{p(1-p)}$. Then we have just one choice of coupling. For this coupling, $W_{1}^{\pi_{1}}-W_{2}^{\pi_{2}}$ will have two atoms, and it will be equal to $-\sqrt{p(1-p)}$ on the diagonal, and $1-\sqrt{p(1-p)}$ off the diagonal. It is then not difficult to see that $\left\|W_{1}-W_{2}\right\|_{2 \rightarrow 2}$ is equal to the largest eigenvalue (in absolute value) of the matrix

$$
\left(\begin{array}{cc}
-p a & (1-p)(1-a) \\
p(1-a) & -(1-p) a
\end{array}\right)
$$

The trace of this matrix is $-a$, and the determinant is

$$
p(1-p) a^{2}-p(1-p)(1-a)^{2}=p(1-p)(2 a-1)<0 .
$$

Here we used that $a=\sqrt{p(1-p)}<1 / 2$. We then have that the two eigenvalues of the matrix have opposite signs, and their sum is $-a$, which implies that the negative one must be less than $-a$; i.e., it must have larger absolute value than $a$.

On the other hand, clearly $\left\|W_{1}\right\|_{2 \rightarrow 2}=a$, and $\left\|W_{2}\right\|_{2 \rightarrow 2}$ is equal to the largest eigenvalue (in absolute value) of the matrix

$$
\left(\begin{array}{cc}
0 & 1-p \\
p & 0
\end{array}\right)
$$

which can easily be seen to be equal to $\sqrt{p(1-p)}=a$.

Therefore, if we extend $W_{1}$ and $W_{2}$ by spaces of total measure at least 1 , and couple each $\Omega_{i}$ to the extension, then

$$
\left\|\widetilde{W}_{1}-\widetilde{W}_{2}\right\|_{2 \rightarrow 2}=\max \left\{\left\|\widetilde{W}_{1}\right\|_{2 \rightarrow 2},\left\|\widetilde{W}_{2}\right\|_{2 \rightarrow 2}\right\}=a .
$$

Therefore, by extending, we can obtain a better coupling.

Finally, note that in (2.1), if we multiply the measure of the underlying space by $c$, then the first term is multiplied by $c$, the second by $c^{3 / 4}$, and the third by $c^{2 / 3}$. We can therefore take $c$ large enough so that the maximum in the term is dominated by $\left\|W_{1}-W_{2}\right\|_{2 \rightarrow 2}$. Therefore, we obtain that also for minimizing $d_{2 \rightarrow 2}$, we obtain a better coupling if we extend by trivial extensions than if we do not. 
Next, we would like to prove Theorem 2.15. Before doing so, we note that the distance $\delta_{\diamond}$ is well defined and finite for signed graphexes as well, provided the graphon part is bounded in the $L^{\infty}$ norm. As the reader may easily verify, this immediately follows from the following lemma, which is an easy corollary to Proposition 2.4.

Lemma 3.12. Let $\mathbb{W}$ be a signed graphex with bounded graphon part, and let $0<D<\infty$. Then the set $\left\{D_{|\mathbb{W}|}>D\right\}$ has finite measure, and $\left.\mathbb{W}\right|_{\left\{D_{|\mathbb{W}|} \leq D\right\}}$ is integrable and hence in $L^{1} \cap L^{2}$.

Proof. Let $\mathbb{W}=(W, S, I, \boldsymbol{\Omega})$ with $\boldsymbol{\Omega}=(\Omega, \mathcal{F}, \mu)$, and assume that $\|W\|_{\infty} \leq K$. If $K \leq 1$, the lemma follows from Proposition 2.4 applied to $\mathbb{W}^{\prime}=|\mathbb{W}|$. Otherwise, we define $\mathbb{W}^{\prime}=\left(W^{\prime}, S^{\prime}, I^{\prime}, \boldsymbol{\Omega}\right)$ where $W^{\prime}=|W| / K, S^{\prime}=|S|$, and $I^{\prime}=|I|$. Applying Proposition 2.4 to this graphex, and noting that $\frac{1}{K} D_{|\mathbb{W}|} \leq D_{\mathbb{W}^{\prime}} \leq D_{|\mathbb{W}|}$, we see that

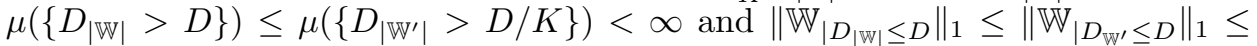
$K\left\|\mathbb{W}_{\mid D_{\mathbb{W}^{\prime}} \leq D}^{\prime}\right\|_{1}<\infty$, as claimed.

To prove Theorem 2.15, we need to establish the triangle inequality. The reason it is not obvious is because when we decrease the measure on the underlying set, the $\delta_{2 \rightarrow 2}$ distance can increase. In the following lemma, we show that although it can increase under restrictions to subsets or decreasing of the underlying measure, it cannot increase too much.

Lemma 3.13. Let $\mathbb{W}_{1}=\left(W_{1}, S_{1}, I_{1}, \boldsymbol{\Omega}\right)$ and $\mathbb{W}_{2}=\left(W_{2}, S_{2}, I_{2}, \boldsymbol{\Omega}\right)$ be signed graphexes in $L^{1} \cap L^{2}$, let $\boldsymbol{\Omega}=(\Omega, \mathcal{F}, \mu)$, and let

(1) $\left\|W_{1}-W_{2}\right\|_{2 \rightarrow 2, \mu}=a$,

(2) $\left\|D_{\mathbb{W}_{1}}-D_{\mathbb{W}_{2}}\right\|_{2, \mu}=b$, and

(3) $\left|\rho\left(\mathbb{W}_{1}\right)-\rho\left(\mathbb{W}_{2}\right)\right|=c$.

If $\mathbb{W}_{1}^{\prime}=\left(W_{1}, S_{1}, I_{1}, \boldsymbol{\Omega}^{\prime}\right)$ and $\mathbb{W}_{2}^{\prime}=\left(W_{2}, S_{2}, I_{2}, \boldsymbol{\Omega}^{\prime}\right)$ where $\boldsymbol{\Omega}^{\prime}=\left(\Omega, \mathcal{F}, \mu^{\prime}\right)$ for some measure $\mu^{\prime}$ such that $\mu-r \leq \mu^{\prime} \leq \mu$ for some $r<\infty$, then

(1) $\left\|W_{1}-W_{2}\right\|_{2 \rightarrow 2, \mu^{\prime}} \leq a$,

(2) $\left\|D_{\mathbb{W}_{1}^{\prime}}-D_{\mathbb{W}_{2}^{\prime}}\right\|_{2, \mu^{\prime}} \leq b+a \sqrt{r}$, and

(3) ||$\rho\left(\mathbb{W}_{1}^{\prime}\right)-\rho\left(\mathbb{W}_{2}^{\prime}\right)|-c| \leq 2 b \sqrt{r}+a r$.

We recall that $\mu-r \leq \mu^{\prime} \leq \mu$ if and only if there exists a measurable function $h: \Omega \rightarrow[0,1]$ such that $\mu^{\prime}(B)=\int_{B} h d \mu$ for all measurable sets $B$, and $\|1-h\|_{1, \mu} \leq$ $r<\infty$. An interesting special case is the case where $h$ is the characteristic function of $\Omega \backslash R$ for a set $R$ of measure $r$, in which case $\mathbb{W}_{i}^{\prime}$ is the restriction of $\mathbb{W}$ to $\Omega \backslash R$, after neglecting points outside the degree support.

Proof. To show property (1), let $f, g$ be such that $\|f\|_{2, \mu^{\prime}}=\|g\|_{2, \mu^{\prime}}=1$. In other words,

$$
1=\int_{\Omega} f^{2} d \mu^{\prime}=\int_{\Omega} h f^{2} d \mu,
$$

and

$$
1=\int_{\Omega} g^{2} d \mu^{\prime}=\int_{\Omega} h g^{2} d \mu .
$$


Let $U=W_{1}-W_{2}$. We then have

$$
\begin{aligned}
\left|\int_{\Omega \times \Omega} f(x) U(x, y) g(y) d\left(\mu^{\prime} \times \mu^{\prime}\right)\right| & =\left|\int_{\Omega \times \Omega} f(x) U(x, y) g(y) h(x) h(y) d(\mu \times \mu)\right| \\
& \leq\|U\|_{2 \rightarrow 2, \mu}\|f h\|_{2, \mu}\|g h\|_{2, \mu} .
\end{aligned}
$$

We also have

$$
\|f h\|_{2, \mu}^{2}=\int_{\Omega} f(x)^{2} h(x)^{2} d \mu(x) \leq \int_{\Omega} f(x)^{2} h(x) d \mu(x)=1 .
$$

Similarly, $\|g h\|_{2, \mu} \leq 1$. Therefore,

$$
\left|\int_{\Omega \times \Omega} f(x) U(x, y) g(y) d\left(\mu^{\prime} \times \mu^{\prime}\right)\right| \leq a
$$

Since this holds for any $f, g$ such that $\|f\|_{2, \mu^{\prime}}=\|g\|_{2, \mu^{\prime}}=1$, we have $\|U\|_{2 \rightarrow 2, \mu^{\prime}} \leq$ $\|U\|_{2 \rightarrow 2, \mu}=a$.

For (2), let

$$
D_{i}(x)=D_{\mathbb{W}_{i}}(x)-D_{\mathbb{W}_{i}^{\prime}}(x)=\int_{\Omega} W_{i}(x, y)(1-h(y)) d \mu(y) .
$$

Then

$$
\begin{aligned}
\left\|D_{\mathbb{W}_{1}^{\prime}}-D_{\mathbb{W}_{2}^{\prime}}\right\|_{2, \mu^{\prime}} \leq\left\|D_{\mathbb{W}_{1}^{\prime}}-D_{\mathbb{W}_{2}^{\prime}}\right\|_{2, \mu} \\
=\sup _{g:\|g\|_{2, \mu}=1} \int_{\Omega}\left(D_{\mathbb{W}_{1}^{\prime}}-D_{\mathbb{W}_{2}^{\prime}}\right)(x) g(x) d \mu(x) \\
=\sup _{g:\|g\|_{2, \mu}=1} \int_{\Omega}\left(\left(D_{\mathbb{W}_{1}}-D_{\mathbb{W}_{2}}\right)(x) g(x)-\left(D_{1}-D_{2}\right)(x) g(x)\right) d \mu(x) \\
=\sup _{g:\|g\|_{2, \mu}=1}\left(\int_{\Omega}\left(D_{\mathbb{W}_{1}}-D_{\mathbb{W}_{2}}\right)(x) g(x) d \mu(x)\right. \\
\left.\quad-\int_{\Omega \times \Omega} g(x)\left(W_{1}-W_{2}\right)(x, y)(1-h(y)) d(\mu \times \mu)\right) \\
\leq b+a\|g\|_{2, \mu}\|1-h\|_{2, \mu} \leq b+a \sqrt{r} .
\end{aligned}
$$


To prove (3), we use that

$$
\begin{aligned}
\rho\left(\mathbb{W}_{i}^{\prime}\right)= & \int_{\Omega \times \Omega} h(x) W_{i}(x, y) h(y) d \mu(x) d \mu(y)+2 \int_{\Omega} S_{i}(x) h(x) d \mu(x)+I_{i} \\
= & \rho\left(\mathbb{W}_{i}\right)-\int_{\Omega \times \Omega}(1-h(x)) W_{i}(x, y) d \mu(x) d \mu(y) \\
& \quad-\int_{\Omega \times \Omega} W_{i}(x, y)(1-h(y)) d \mu(x) d \mu(y) \\
& +\int_{\Omega \times \Omega}(1-h(x)) W_{i}(x, y)(1-h(y)) d \mu(x) d \mu(y) \\
& \quad-2 \int_{\Omega}(1-h(x)) S(x) d \mu(x) \\
= & \rho\left(\mathbb{W}_{i}\right)-2 \int_{\Omega}(1-h(x)) D_{\mathbb{W}_{i}}(x) d \mu(x) \\
& +\int_{\Omega \times \Omega}(1-h(x)) W_{i}(x, y)(1-h(y)) d \mu(x) d \mu(y) .
\end{aligned}
$$

Therefore,

$$
\begin{aligned}
& || \rho\left(\mathbb{W}_{1}^{\prime}\right)-\rho\left(\mathbb{W}_{2}^{\prime}\right)|-| \rho\left(\mathbb{W}_{1}\right)-\rho\left(\mathbb{W}_{2}\right)|| \\
& \leq \mid 2 \int_{\Omega}(1-h(x))\left(D_{\mathbb{W}_{1}}-D_{\mathbb{W}_{2}}\right)(x) d \mu(x) \\
& \quad-\int_{\Omega \times \Omega}(1-h(x))\left(W_{1}-W_{2}\right)(x, y)(1-h(y)) d \mu(x) d \mu(y) \mid \\
& \leq 2 b \sqrt{r}+a r .
\end{aligned}
$$

Here we used the fact that $\|1-h\|_{2} \leq \sqrt{\|1-h\|_{1}} \leq \sqrt{r}$. This implies the claim. $\delta_{\diamond}$.

We also use the following equivalent representation of the weak kernel distance

Lemma 3.14. For $i=1,2$, let $\mathbb{W}_{i}$ be graphexes over $\boldsymbol{\Omega}_{i}=\left(\Omega_{i}, \mathcal{F}_{i}, \mu_{i}\right)$, and let $\mathbb{W}_{i}^{\prime}$ be trivial extensions of $\mathbb{W}_{i}$ to $\sigma$-finite measure spaces $\boldsymbol{\Omega}_{i}^{\prime}=\left(\Omega_{i}^{\prime}, \mathcal{F}_{i}^{\prime}, \mu_{i}^{\prime}\right)$ with $\mu_{i}^{\prime}\left(\Omega_{i}^{\prime}\right)=\infty$. Then $\delta_{\diamond}\left(\mathbb{W}_{1}, \mathbb{W}_{2}\right)=\widetilde{\delta}_{\diamond}\left(\mathbb{W}_{1}^{\prime}, \mathbb{W}_{2}^{\prime}\right)$, where $\widetilde{\delta}_{\diamond}\left(\mathbb{W}_{1}^{\prime}, \mathbb{W}_{2}^{\prime}\right)$ is defined as the infimum over all $c$ such that there exists a measure $\mu^{\prime}$ over $\Omega_{1}^{\prime} \times \Omega_{2}^{\prime}$ obeying the conditions

(1) $\mu_{i}^{\prime}-c^{2} \leq\left(\mu^{\prime}\right)^{\pi_{i}} \leq \mu_{i}^{\prime}$ for $i=1,2$, and

(2) $d_{2 \rightarrow 2}\left(\left(\mathbb{W}_{1}^{\prime}\right)^{\pi_{1}, \mu^{\prime}},\left(\mathbb{W}_{2}^{\prime}\right)^{\pi_{2}, \mu^{\prime}}\right) \leq c$.

Proof. Let $\mu^{\prime}$ and $c$ be such that they obey the conditions in the statement of the lemma. For $i=1,2$, let $\widetilde{\mu}_{i}$ be the restriction of $\left(\mu^{\prime}\right)^{\pi_{i}}$ to $\Omega_{i}$, and let $\widetilde{\mathbb{W}}_{i}$ be obtained from $\mathbb{W}_{i}$ by replacing $\mu_{i}$ by $\widetilde{\mu}_{i}$. Then $\mu_{i}-c^{2} \leq \widetilde{\mu}_{i} \leq \mu_{i}$. Furthermore, $\left(\mu^{\prime}\right)^{\pi_{i}}$ extends $\widetilde{\mu}_{i}$ to $\Omega_{i}^{\prime}$, and $\left(\mu^{\prime}\right)^{\pi_{i}}\left(\Omega_{i}^{\prime} \backslash \Omega_{i}\right) \geq \mu_{i}^{\prime}\left(\Omega_{i}^{\prime} \backslash \Omega_{i}\right)-c^{2}=\infty$, showing that this defines an extension by a space of infinite measure. Finally, $\mu^{\prime}$ is a coupling of $\left(\mu^{\prime}\right)^{\pi_{1}}$ and $\left(\mu^{\prime}\right)^{\pi_{2}}$. Together, these facts imply that $\delta_{2 \rightarrow 2}\left(\widetilde{\mathbb{W}}_{1}, \widetilde{\mathbb{W}}_{2}\right) \leq c$, proving that $\delta_{\diamond}\left(\mathbb{W}_{1}, \mathbb{W}_{2}\right) \leq c$. This shows that $\delta_{\diamond}\left(\mathbb{W}_{1}, \mathbb{W}_{2}\right) \leq \widetilde{\delta}_{\diamond}\left(\mathbb{W}_{1}^{\prime}, \mathbb{W}_{2}^{\prime}\right)$.

To prove the reverse inequality, assume that $c$ is such that there are measures $\widetilde{\mu}_{1}$ and $\widetilde{\mu}_{2}$ over $\Omega_{1}$ and $\Omega_{2}$ such that $\delta_{2 \rightarrow 2}\left(\widetilde{\mathbb{W}}_{1}, \widetilde{\mathbb{W}}_{2}\right) \leq c$ and $\mu_{i}-c^{2} \leq \widetilde{\mu}_{i} \leq \mu_{i}$ for 
$i=1,2$, where $\widetilde{\mathbb{W}}_{i}$ is again obtained from $\mathbb{W}_{i}$ by replacing $\mu_{i}$ by $\widetilde{\mu}_{i}$. If we transform $\boldsymbol{\Omega}_{i}^{\prime}$ into a space $\widetilde{\boldsymbol{\Omega}}_{i}^{\prime}$ by setting $\widetilde{\mu}_{i}^{\prime}$ to $\widetilde{\mu}_{i}$ on $\Omega_{i}$, and to $\mu_{i}^{\prime}$ on $\Omega_{i} \backslash \Omega_{i}^{\prime}$, and define $\widetilde{\mathbb{W}}_{i}^{\prime}$ as the trivial extension of $\widetilde{\mathbb{W}}_{i}$ to $\widetilde{\boldsymbol{\Omega}}_{i}^{\prime}$, then Theorem 2.11 implies that $\widetilde{\delta}_{2 \rightarrow 2}\left(\widetilde{\mathbb{W}}_{1}^{\prime}, \widetilde{\mathbb{W}}_{2}^{\prime}\right)=$ $\delta_{2 \rightarrow 2}\left(\widetilde{\mathbb{W}}_{1}, \widetilde{\mathbb{W}}_{2}\right) \leq c$. This means that for all $\varepsilon>0$, there is a coupling $\mu^{\prime}$ of $\widetilde{\mu}_{1}^{\prime}$ and $\widetilde{\mu}_{2}^{\prime}$ such that $d_{2 \rightarrow 2}\left(\left(\mathbb{W}_{1}^{\prime}\right)^{\pi_{1}, \mu^{\prime}},\left(\mathbb{W}_{2}^{\prime}\right)^{\pi_{2}, \mu^{\prime}}\right) \leq c+\varepsilon$. Observing that the bound $\mu_{i}-c^{2} \leq \widetilde{\mu}_{i} \leq \mu_{i}$ and our construction of $\widetilde{\mu}_{i}^{\prime}$ imply that $\mu_{i}^{\prime}-c^{2} \leq \widetilde{\mu}_{i}^{\prime}=\left(\mu^{\prime}\right)^{\pi_{i}} \leq \mu_{i}^{\prime}$, and that $\left(\widetilde{\mathbb{W}}_{i}^{\prime}\right)^{\pi_{i}, \widetilde{\mu}^{\prime}}=\left(\mathbb{W}_{i}^{\prime}\right)^{\pi_{i}, \widetilde{\mu}^{\prime}}$, this shows that $\widetilde{\delta}_{\diamond}\left(\mathbb{W}_{1}^{\prime}, \mathbb{W}_{2}^{\prime}\right) \leq c+\varepsilon$. Since $\varepsilon$ was arbitrary, this shows that $\delta_{\diamond}\left(\mathbb{W}_{1}, \mathbb{W}_{2}\right) \geq \widetilde{\delta}_{\diamond}\left(\mathbb{W}_{1}^{\prime}, \mathbb{W}_{2}^{\prime}\right)$.

We are now ready to prove Theorem 2.15 .

Proof of Theorem 2.15. It is clear that $\delta_{\diamond}$ is symmetric, and that $\delta_{\diamond}(\mathbb{W}, \mathbb{W})=0$. So we have to prove the triangle inequality. By Lemma 3.14, taking trivial extensions of each graphex to a space of infinite measure, it suffices to prove the triangle inequality for $\widetilde{\delta}_{\diamond}$.

Let $\mathbb{W}_{1}, \mathbb{W}_{2}, \mathbb{W}_{3}$ be three graphexes with the usual notation, defined over measure spaces which all have infinite measure. Let $\mu_{12}$ be a measure on $\Omega_{1} \times \Omega_{2}$ that shows that $\widetilde{\delta}_{\diamond}\left(\mathbb{W}_{1}, \mathbb{W}_{2}\right) \leq c_{1}$, let $\mu_{23}$ be a measure on $\Omega_{2} \times \Omega_{3}$ that shows that $\widetilde{\delta}_{\diamond}\left(\mathbb{W}_{1}, \mathbb{W}_{2}\right) \leq c_{2}$, let $\mu_{1}^{\prime}$ and $\mu_{2}^{\prime}$ be the marginals of $\mu_{12}$, and let $\mu_{2}^{\prime \prime}$ and $\mu_{3}^{\prime \prime}$ be the marginals of $\mu_{23}$. We would like to use Lemma 3.9 to create a coupling of $\mu_{1}^{\prime}$ and $\mu_{3}^{\prime \prime}$, but unfortunately, the conditions of the lemma require that $\mu_{2}^{\prime}=\mu_{2}^{\prime \prime}$, which we cannot guarantee. To deal with this problem, we will slightly decrease $\mu_{12}$ and $\mu_{23}$ so that after this perturbation, the second marginal of the first is equal to the first marginal of the second.

Let $\pi_{i j, i}$ be the projection map from $\Omega_{i} \times \Omega_{j}$ to $\Omega_{i}$ for $i, j \in[3]$, and let $\mathbb{W}_{i j, i}=$ $\mathbb{W}_{i}^{\pi_{i j, i}, \mu_{i j}}$. Let $\mu_{2}^{\prime}=\mu_{12}{ }^{\pi_{12,2}}$ and $\mu_{2}^{\prime \prime}=\mu_{23}{ }^{\pi_{23,2}}$. Let $h^{\prime}=\frac{d \mu_{2}^{\prime}}{d \mu_{2}}$ and $h^{\prime \prime}=\frac{d \mu_{2}^{\prime \prime}}{d \mu_{2}}$. Then we can assume that $0 \leq h^{\prime}, h^{\prime \prime} \leq 1,\left\|1-h^{\prime}\right\|_{1, \mu_{2}} \leq c_{1}^{2}$, and $\left\|1-h^{\prime \prime}\right\|_{1, \mu_{2}} \leq c_{2}^{2}$. Let $\widetilde{h}(x)=\min \left(h^{\prime}(x), h^{\prime \prime}(x)\right)$, and let $\widetilde{\mu}_{2}$ be the measure defined by

$$
\widetilde{\mu}_{2}(A)=\int_{A} \widetilde{h} d \mu_{2} .
$$

Then $\left\|h^{\prime}-\widetilde{h}\right\|_{1, \mu_{2}} \leq\left\|1-h^{\prime \prime}\right\|_{1, \mu_{2}} \leq c_{2}^{2}$. For $x \in \Omega_{1} \times \Omega_{2}$, let $h_{12}(x)=\frac{\widetilde{h}\left(\pi_{12,2}(x)\right)}{h^{\prime}\left(\pi_{12,2}(x)\right)} \leq 1$, and let $\widetilde{\mu}_{12}$ be the measure defined by

$$
\widetilde{\mu}_{12}(A)=\int_{A} h_{12}(x) d \mu_{12} .
$$

Note that $\widetilde{\mu}_{12}^{\pi_{12,2}}=\widetilde{\mu}_{2}$. Furthermore, since $\tilde{h}(x) \leq h^{\prime}(x)$,

$$
\begin{aligned}
\int_{\Omega_{1} \times \Omega_{2}}\left(1-h_{12}(x)\right) d \mu_{12}(x) & =\int_{\Omega_{2}}\left(1-\frac{\widetilde{h}(x)}{h^{\prime}(x)}\right) d \mu_{2}^{\prime}(x) \\
& =\int_{\Omega_{2}}\left(1-\frac{\widetilde{h}(x)}{h^{\prime}(x)}\right) h^{\prime}(x) d \mu_{2}(x)=\left\|h^{\prime}-\widetilde{h}\right\|_{1, \mu_{2}} \leq c_{2}^{2} .
\end{aligned}
$$

This means that for any set $A \subseteq \Omega_{1} \times \Omega_{2}$,

$$
\mu_{12}(A)-c_{2}^{2} \leq \widetilde{\mu}_{12}(A) \leq \mu_{12}(A) .
$$

This implies that for any $A \subseteq \Omega_{1}$,

$$
\mu_{1}(A)-c_{1}^{2}-c_{2}^{2} \leq \mu_{12}^{\pi_{12,1}}(A)-c_{2}^{2} \leq \widetilde{\mu}_{12}^{\pi_{12,1}}(A) \leq \mu_{12}^{\pi_{12,1}}(A) \leq \mu_{1}(A) .
$$


We similarly construct $\widetilde{\mu}_{23}$ and $\Omega_{2} \times \Omega_{3}$ so that $\widetilde{\mu}_{23}^{\pi_{23,2}}=\widetilde{\mu}_{2}$ and for any set $A \subseteq \Omega_{2} \times \Omega_{3}$,

$$
\mu_{23}(A)-c_{1}^{2} \leq \widetilde{\mu}_{23}(A) \leq \mu_{23}(A),
$$

which implies that for any $A \subseteq \Omega_{3}$,

$$
\mu_{3}(A)-c_{1}^{2}-c_{2}^{2} \leq \mu_{23}^{\pi_{23,3}}(A)-c_{1}^{2} \leq \widetilde{\mu}_{23}^{\pi_{23,3}}(A) \leq \mu_{23}^{\pi_{23,3}}(A) \leq \mu_{3}(A) .
$$

Let $\widetilde{\mu}_{1}=\widetilde{\mu}_{12}^{\pi_{12,1}}$ and $\widetilde{\mu}_{3}=\widetilde{\mu}_{23}^{\pi_{23,3}}$, and note that $\widetilde{\mu}_{12}$ is a coupling of $\widetilde{\mu}_{1}$ and $\widetilde{\mu}_{2}, \widetilde{\mu}_{23}$ is a coupling of $\widetilde{\mu}_{2}$ and $\widetilde{\mu}_{3}$, and $\mu_{1}-c_{1}^{2}-c_{2}^{2} \leq \widetilde{\mu}_{1} \leq \mu_{1}$ and $\mu_{3}-c_{1}^{2}-c_{2}^{2} \leq \widetilde{\mu}_{3} \leq \mu_{3}$.

Let $\widetilde{\mathbb{W}}_{i}$ be equal to $\mathbb{W}_{i}$ but with the measure $\mu_{i}$ replaced by $\widetilde{\mu}_{i}$. Fix $\varepsilon>0$. By Lemma 3.9 , there exists a measure $\widetilde{\mu}_{13}$ on $\Omega_{1} \times \Omega_{3}$ such that $\widetilde{\mu}_{13}^{\pi_{13,1}}=\widetilde{\mu}_{1}$ and $\widetilde{\mu}_{13}^{\pi_{13,3}}=\widetilde{\mu}_{3}$, and we have

$$
\Delta_{2 \rightarrow 2}\left(\widetilde{\mathbb{W}}_{1}^{\pi_{13,1}}, \widetilde{\mathbb{W}}_{3}^{\pi_{13,3}}\right) \leq \Delta_{2 \rightarrow 2}\left(\widetilde{\mathbb{W}}_{1}^{\pi_{12,1}}, \widetilde{\mathbb{W}}_{2}^{\pi_{12,2}}\right)+\Delta_{2 \rightarrow 2}\left(\widetilde{\mathbb{W}}_{2}^{\pi_{23,2}}, \widetilde{\mathbb{W}}_{3}^{\pi_{23,3}}\right)+(\varepsilon, \varepsilon, \varepsilon) .
$$

Note that by the above inequalities,

$$
\mu_{1}-\left(c_{1}+c_{2}\right)^{2} \leq \mu_{1}-c_{1}^{2}-c_{2}^{2} \leq \widetilde{\mu}_{1}=\widetilde{\mu}_{13}^{\pi_{13,1}} \leq \mu_{1}
$$

and

$$
\mu_{3}-\left(c_{1}+c_{2}\right)^{2} \leq \mu_{3}-c_{1}^{2}-c_{2}^{2} \leq \widetilde{\mu}_{3}=\widetilde{\mu}_{13}^{\pi_{13,3}} \leq \mu_{3}
$$

By Lemma 3.13,

$$
\begin{aligned}
& \left\|\widetilde{W}_{1}^{\pi_{12,1}}-\widetilde{W}_{2}^{\pi_{12,2}}\right\|_{2 \rightarrow 2, \widetilde{\mu}_{12}} \leq\left\|W_{12,1}-W_{12,2}\right\|_{2 \rightarrow 2, \mu_{12}} \leq c_{1}, \\
& \left\|D_{\widetilde{\mathbb{W}}_{1}^{\pi_{12,1}}}-D_{\widetilde{\mathbb{W}}_{2}^{\pi_{12,2}}}\right\|_{2, \widetilde{\mu}_{12}} \leq\left\|D_{\mathbb{W}_{12,1}}-D_{\mathbb{W}_{12,2}}\right\|_{2, \mu_{12}}+\left\|W_{12,1}-W_{12,2}\right\|_{2 \rightarrow 2, \mu_{12}} c_{2} \\
& \leq c_{1}^{2}+c_{1} c_{2} \text {, }
\end{aligned}
$$

and finally

$$
\begin{aligned}
\left|\rho\left(\widetilde{\mathbb{W}}_{1}^{\pi_{12,1}}\right)-\rho\left(\widetilde{\mathbb{W}}_{2}^{\pi_{12,2}}\right)\right| \leq & \left|\rho\left(\mathbb{W}_{12,1}\right)-\rho\left(\mathbb{W}_{12,2}\right)\right|+2\left\|D_{\mathbb{W}_{12,1}}-D_{\mathbb{W}_{12,2}}\right\|_{2, \mu_{12}} c_{2} \\
& +\left\|W_{12,1}-W_{12,2}\right\|_{2 \rightarrow 2, \mu_{12}} c_{2}^{2} \\
\leq & c_{1}^{3}+2 c_{1}^{2} c_{2}+c_{1} c_{2}^{2}
\end{aligned}
$$

To summarize, this means that

$$
\Delta_{2 \rightarrow 2}\left(\widetilde{\mathbb{W}}_{1}^{\pi_{12,1}}, \widetilde{\mathbb{W}}_{2}^{\pi_{12,2}}\right) \leq\left(c_{1}, c_{1}^{2}+c_{1} c_{2}, c_{1}^{3}+2 c_{1}^{2} c_{2}+c_{1} c_{2}^{2}\right) .
$$

Similarly,

$$
\Delta_{2 \rightarrow 2}\left(\widetilde{\mathbb{W}}_{2}^{\pi_{23,2}}, \widetilde{\mathbb{W}}_{3}^{\pi_{23,3}}\right) \leq\left(c_{2}, c_{2}^{2}+c_{1} c_{2}, c_{2}^{3}+2 c_{1} c_{2}^{2}+c_{1}^{2} c_{2}\right) .
$$

Therefore,

$$
\begin{gathered}
\left\|\widetilde{W}_{1}^{\pi_{13,1}}-\widetilde{W}_{3}^{\pi_{13,3}}\right\|_{2 \rightarrow 2, \widetilde{\mu}_{13}} \leq c_{1}+c_{2}+\varepsilon \\
\left\|D_{\widetilde{\mathbb{W}}_{1}}-D_{\widetilde{\mathbb{W}}_{3}}\right\|_{2, \widetilde{\mu}_{13}} \leq c_{1}^{2}+c_{1} c_{2}+c_{2}^{2}+c_{1} c_{2}+\varepsilon=\left(c_{1}+c_{2}\right)^{2}+\varepsilon
\end{gathered}
$$

and finally

$$
\left|\rho\left(\widetilde{\mathbb{W}}_{1}\right)-\rho\left(\widetilde{\mathbb{W}}_{3}\right)\right| \leq c_{1}^{3}+2 c_{1}^{2} c_{2}+c_{1} c_{2}^{2}+c_{2}^{3}+2 c_{1} c_{2}^{2}+c_{1}^{2} c_{2}+\varepsilon=\left(c_{1}+c_{2}\right)^{3}+\varepsilon .
$$

Since this can be done for any $\varepsilon>0$, this completes the proof that $\widetilde{\delta}_{\diamond}$ is a metric. With the help of Lemma 3.14 the triangle inequality for $\widetilde{\delta}_{\diamond}$ implies that for $\delta_{\diamond}$.

Next we prove Proposition 2.17, as well the following version for signed graphexes.

Proposition 3.15. Fix $B, C, D<\infty$. Then $\delta_{\diamond}$ and $\delta_{2 \rightarrow 2}$ define the same topology on the space of $(B, C, D)$-bounded signed graphexes. 
To prove these propositions, we need a lemma complementing the bounds from Lemma 3.13. Recall that in Lemma 3.13, we showed that the distance between two graphexes defined on the same measure space cannot increase too much when we decrease of the underlying measure. Our next lemma shows that if the graphexes involved are signed graphexes that are $(B, C, D)$-bounded, we can also go in the other direction.

Lemma 3.16. Let $\mathbb{W}_{i}=\left(W_{i}, S_{i}, I_{i}, \boldsymbol{\Omega}\right)$, for $i=1,2$, be $(B, C, D)$-bounded signed graphexes on the same measure space $\Omega$, and let $\mu^{\prime}, r, \mathbb{W}_{1}^{\prime}$, and $\mathbb{W}_{2}^{\prime}$ be as in Lemma 3.13. Then

(1) $\left\|W_{1}-W_{2}\right\|_{2 \rightarrow 2, \mu^{\prime}} \leq\left\|W_{1}-W_{2}\right\|_{2 \rightarrow 2, \mu} \leq\left\|W_{1}-W_{2}\right\|_{2 \rightarrow 2, \mu^{\prime}}+4 \sqrt{B D r}$,

(2) $\left|\left\|D_{\mathbb{W}_{1}}-D_{\mathbb{W}_{2}}\right\|_{2, \mu}^{2}-\left\|D_{\mathbb{W}_{1}^{\prime}}-D_{\mathbb{W}_{2}^{\prime}}\right\|_{2, \mu^{\prime}}^{2}\right| \leq\left(4 D^{2}+8 B C\right) r$, and

(3) ||$\rho\left(\mathbb{W}_{1}^{\prime}\right)-\rho\left(\mathbb{W}_{2}^{\prime}\right)|-| \rho\left(\mathbb{W}_{1}\right)-\rho\left(\mathbb{W}_{2}\right)|| \leq 4 D r$.

Proof. Let $U=W_{1}-W_{2}$. Then for any $f, g$ with $\|f\|_{2, \mu}=\|g\|_{2, \mu}=1$,

$$
\begin{aligned}
\int_{\Omega \times \Omega} & f(x) U(x, y) g(y) d \mu(x) d \mu(y) \\
= & \int_{\Omega \times \Omega} f(x) h(x) U(x, y) h(y) g(y) d \mu(x) d \mu(y) \\
& +\int_{\Omega \times \Omega} f(x)((1-h(x)) U(x, y) h(y)) g(y) d \mu(x) d \mu(y) \\
& +\int_{\Omega \times \Omega} f(x) U(x, y)(1-h(y)) g(y) d \mu(x) d \mu(y) .
\end{aligned}
$$

We have

$$
\begin{aligned}
\int_{\Omega \times \Omega} & f(x) h(x) U(x, y) h(y) g(y) d \mu(x) d \mu(y) \\
& =\int_{\Omega \times \Omega} f(x) U(x, y) g(y) d \mu^{\prime}(x) d \mu^{\prime}(y) \leq\|f\|_{2, \mu^{\prime}}\|U\|_{2 \rightarrow 2, \mu^{\prime}}\|g\|_{2, \mu^{\prime}} \\
& \leq\|f\|_{2, \mu}\|U\|_{2 \rightarrow 2, \mu^{\prime}}\|g\|_{2, \mu} \leq\|U\|_{2 \rightarrow 2, \mu^{\prime}} .
\end{aligned}
$$

Furthermore,

$$
\begin{aligned}
\int_{\Omega \times \Omega} f(x) U(x, y)(1-h(y)) & g(y) d \mu(x) d \mu(y) \\
& \leq\|f\|_{2, \mu} \int_{\Omega}\left\|U_{y}\right\|_{2, \mu}(1-h(y))|g(y)| d \mu(y) \\
& \leq\|f\|_{2, \mu} 2 \sqrt{B D} \int_{\Omega}(1-h(y))|g(y)| d \mu(y) \\
& \leq 2 \sqrt{B D}\|f\|_{2, \mu}\|1-h\|_{2, \mu}\|g\|_{2, \mu} \leq 2 \sqrt{B D r} .
\end{aligned}
$$

Here we used the fact that $\|U\|_{\infty} \leq 2 B$ and $\left\|D_{|U|, \mu}\right\|_{\infty} \leq 2 D$, which implies that $\left\|U_{y}\right\|_{2, \mu} \leq 2 \sqrt{B D}$. Analogously, we have

$$
\begin{aligned}
\int_{\Omega \times \Omega} f(x)((1-h(x)) U(x, y) & h(y)) g(y) d \mu(x) d \mu(y) \\
\leq & 2 \sqrt{B D}\|f\|_{2, \mu}\|1-h\|_{2, \mu}\|h g\|_{2, \mu} \leq 2 \sqrt{B D r} .
\end{aligned}
$$


Adding this all up, we have

$$
\int_{\Omega \times \Omega} f(x) U(x, y) g(y) d \mu(x) d \mu(y) \leq\|U\|_{2 \rightarrow 2, \mu^{\prime}}+4 \sqrt{B D r} .
$$

This proves the upper bound in the first claim. The lower bound follows from Lemma 3.13.

To prove the second claim, we observe that

$$
\begin{aligned}
0 & \leq \int_{\Omega}\left(D_{\mathbb{W}_{1}}(x)-D_{\mathbb{W}_{2}}(x)\right)^{2} d \mu(x)-\int_{\Omega}\left(D_{\mathbb{W}_{1}}(x)-D_{\mathbb{W}_{2}}(x)\right)^{2} d \mu^{\prime}(x) \\
& =\int_{\Omega}\left(D_{\mathbb{W}_{1}}(x)-D_{\mathbb{W}_{2}}(x)\right)^{2}(1-h(x)) d \mu(x) \leq \int_{\Omega} 4 D^{2}(1-h(x)) d \mu(x) \leq 4 D^{2} r .
\end{aligned}
$$

Furthermore, since $\|U\|_{\infty} \leq 2 B$, we have that for any $x \in \Omega$,

$$
\begin{aligned}
\left|\left(D_{\mathbb{W}_{1}}(x)-D_{\mathbb{W}_{1}^{\prime}}(x)\right)-\left(D_{\mathbb{W}_{2}}(x)-D_{\mathbb{W}_{2}^{\prime}}(x)\right)\right| \\
=\left|\int_{\Omega}(1-h(y)) U(x, y) d \mu(y)\right| \leq 2 B r .
\end{aligned}
$$

Therefore,

$$
\begin{aligned}
& \left|\int_{\Omega}\left(D_{\mathbb{W}_{1}}(x)-D_{\mathbb{W}_{2}}(x)\right)^{2} d \mu^{\prime}(x)-\int_{\Omega}\left(D_{\mathbb{W}_{1}^{\prime}}(x)-D_{\mathbb{W}_{2}^{\prime}}(x)\right)^{2} d \mu^{\prime}(x)\right| \\
& =\mid \int_{\Omega}\left(\left(D_{\mathbb{W}_{1}}(x)-D_{\mathbb{W}_{2}}(x)\right)-\left(D_{\mathbb{W}_{1}^{\prime}}(x)-D_{\mathbb{W}_{2}^{\prime}}(x)\right)\right) \\
& \quad\left(\left(D_{\mathbb{W}_{1}}(x)-D_{\mathbb{W}_{2}}(x)\right)+\left(D_{\mathbb{W}_{1}^{\prime}}(x)-D_{\mathbb{W}_{2}^{\prime}}(x)\right)\right) d \mu^{\prime}(x) \mid \\
& \quad \leq 2 B r \int_{\Omega}\left(\left|D_{\mathbb{W}_{1}}(x)\right|+\left|D_{\mathbb{W}_{2}}(x)\right|+\left|D_{\mathbb{W}_{1}^{\prime}}(x)\right|+\left|D_{\mathbb{W}_{2}^{\prime}}(x)\right|\right) d \mu^{\prime}(x) \leq 8 B C r .
\end{aligned}
$$

Combining these two inequalities proves the second claim.

To prove the third claim, we use the following bound, where the first inequality was already established when proving the last claim of Lemma 3.13:

$$
\begin{aligned}
|| \rho\left(\mathbb{W}_{1}^{\prime}\right)- & \rho\left(\mathbb{W}_{2}^{\prime}\right)|-| \rho\left(\mathbb{W}_{1}\right)-\rho\left(\mathbb{W}_{2}\right)|| \\
\leq & \mid 2 \int_{\Omega}(1-h(x))\left(D_{\mathbb{W}_{1}}-D_{\mathbb{W}_{2}}\right)(x) d \mu(x) \\
& \quad-\int_{\Omega \times \Omega}(1-h(x))\left(W_{1}-W_{2}\right)(x, y)(1-h(y)) d \mu(x) d \mu(y) \mid \\
= & \mid 2 \int_{\Omega}(1-h(x))\left(S_{1}-S_{2}\right)(x) d \mu(x) \\
& \quad+\int_{\Omega \times \Omega}(1-h(x))\left(W_{1}-W_{2}\right)(x, y)(2-1+h(y)) d \mu(x) d \mu(y) \mid \\
\leq & 2 \int_{\Omega}(1-h(x))\left(D_{\left|\mathbb{W}_{1}\right|}+D_{\left|\mathbb{W}_{2}\right|}\right)(x) \leq 4 D r .
\end{aligned}
$$

Our next lemma is an easy corollary to Lemma 3.16, and in turn immediately implies Proposition 2.17 and 3.15. 
Lemma 3.17. Suppose $\mathbb{W}_{i}=\left(W_{i}, S_{i}, I_{i}, \boldsymbol{\Omega}_{i}\right)$, for $i=1,2$, are signed graphexes with $\left\|W_{i}\right\|_{\infty} \leq B$ and $\left\|D_{|\mathbb{W}|}\right\|_{\infty} \leq D$, and let $\delta_{\diamond}\left(\mathbb{W}_{1}, \mathbb{W}_{2}\right) \leq \varepsilon$. Then $\left|\rho\left(W_{1}\right)-\rho\left(W_{2}\right)\right| \leq$ $\varepsilon^{3}+4 \varepsilon^{2} D$. If, in addition, $\|W\|_{i} \leq C$, then $\delta_{2 \rightarrow 2}\left(\mathbb{W}_{1}, \mathbb{W}_{2}\right) \leq f(\varepsilon)$, where

$$
f(\varepsilon)=\max \left\{\varepsilon+4 \varepsilon \sqrt{B D},\left(\varepsilon^{2}+2 \varepsilon \sqrt{D^{2}+2 B C}\right)^{1 / 2},\left(\varepsilon^{3}+4 \varepsilon^{2} D\right)^{1 / 3}\right\}
$$

Proof. We first note that by Lemma $3.12,\left\|\mathbb{W}_{i}\right\|_{1}<\infty$, so even without the assumption that $\left\|W_{i}\right\|_{1} \leq C$, we always have that $\left\|W_{i}\right\|_{1} \leq C$ for some $C<\infty$.

Next, let $\boldsymbol{\Omega}_{i}=\left(\Omega_{i}, \mathcal{F}_{i}, \mu_{i}\right)$, for $i=1,2$, and let $c>\varepsilon$. By the definition of $\delta_{\diamond}$, there exist measures $\widetilde{\mu}_{i}$ such that $\widetilde{\mu}_{i} \leq \mu_{i}$ and $\delta_{i}=\mu_{i}\left(\Omega_{i}\right)-\widetilde{\mu}_{i}\left(\Omega_{i}\right) \leq c^{2}$ and such that $\delta_{2 \rightarrow 2}\left(\widetilde{\mathbb{W}}_{1}, \widetilde{\mathbb{W}}_{2}\right)<c$ for the signed graphexes $\widetilde{\mathbb{W}}_{i}$ obtained from $\mathbb{W}_{i}$ by replacing $\mu_{i}$ by $\widetilde{\mu}_{i}$.

Consider an arbitrary $\sigma$-finite space $\boldsymbol{\Omega}_{i}^{\prime \prime}$ of infinite measure, and two intervals $J_{i}$ of length $c-\delta_{i}$. Define $\boldsymbol{\Omega}_{i}^{\prime}$ by appending $\boldsymbol{\Omega}_{i}^{\prime \prime}$ and the interval $J_{i}$ equipped with the Lebesgue measure to $\boldsymbol{\Omega}_{i}$, and define $\widetilde{\boldsymbol{\Omega}}_{i}^{\prime}$ by appending the same spaces, except that we equip $J_{i}$ with the zero measure. By Lemma 3.10 and the definition of $\delta_{2 \rightarrow 2}$, we then have that $\widetilde{\delta}_{2 \rightarrow 2}\left(\widetilde{\mathbb{W}}_{1}^{\prime}, \widetilde{\mathbb{W}}_{2}^{\prime}\right)=\delta_{2 \rightarrow 2}\left(\widetilde{\mathbb{W}}_{1}, \widetilde{\mathbb{W}}_{2}\right)<c$, where $\widetilde{\mathbb{W}}_{i}^{\prime}$ are the trivial extensions to $\widetilde{\boldsymbol{\Omega}}_{i}^{\prime}$. Furthermore, by our construction, $\boldsymbol{\Omega}_{i}^{\prime}=\left(\Omega_{i}, \mathcal{F}_{i}^{\prime}, \mu_{i}^{\prime}\right)$ and $\widetilde{\boldsymbol{\Omega}}_{i}^{\prime}=\left(\widetilde{\Omega}_{i}, \widetilde{\mathcal{F}}_{i}^{\prime}, \widetilde{\mu}_{i}^{\prime}\right)$ are such that $\left(\Omega_{i}^{\prime}, \mathcal{F}_{i}^{\prime}\right)=\left(\widetilde{\Omega}_{i}, \widetilde{\mathcal{F}}_{i}^{\prime}\right), \widetilde{\mu}_{i}^{\prime} \leq \mu_{i}^{\prime}$, and $\mu_{i}^{\prime}\left(\Omega_{i}^{\prime}\right)-\widetilde{\mu}_{i}^{\prime}\left(\Omega_{i}^{\prime}\right)=c^{2}$.

Given a coupling $\widetilde{\mu}^{\prime}$ of $\widetilde{\mu}_{1}^{\prime}$ and $\widetilde{\mu}_{2}^{\prime}$, let $\widetilde{\mathbb{U}}_{i}^{\prime}$ be the pullback of $\widetilde{\mathbb{W}}_{i}^{\prime}$ under the coordinate projections onto $\Omega_{i}^{\prime}$. By the definition of the distance $\widetilde{\delta}_{2 \rightarrow 2}$, we can find a coupling $\widetilde{\mu}^{\prime}$ such that $d_{2 \rightarrow 2}\left(\widetilde{\mathbb{U}}_{1}^{\prime}, \widetilde{\mathbb{U}}_{2}^{\prime}\right) \leq c^{2}$. Choose a coupling $\mu^{\prime}$ of $\mu_{1}^{\prime}$ and $\mu_{2}^{\prime}$ by coupling $\mu_{i}^{\prime}-\widetilde{\mu}_{i}^{\prime}$ arbitrarily. Then $\mu^{\prime}-c \leq \widetilde{\mu}^{\prime} \leq \mu^{\prime}$. Defining $\mathbb{U}_{i}^{\prime}$ to be the pullbacks of $\mathbb{W}_{i}^{\prime}$ under the coordinate projections onto $\Omega_{i}^{\prime}$, we may then apply Lemma 3.16 with $r=c^{2}$ to conclude that $\left|\rho\left(\mathbb{W}_{1}\right)-\rho\left(\mathbb{W}_{2}\right)\right|=\left|\rho\left(\mathbb{U}_{1}^{\prime}\right)-\rho\left(\mathbb{U}_{2}^{\prime}\right)\right| \leq c^{3}+4 c^{2} D$ and

$$
\begin{aligned}
\delta_{2 \rightarrow 2}\left(\mathbb{W}_{1}, \mathbb{W}_{2}\right) & \leq d_{2 \rightarrow 2}\left(\mathbb{U}_{1}^{\prime}, \mathbb{U}_{2}^{\prime}\right) \\
& \leq \max \left\{c+4 c \sqrt{B D},\left(c^{2}+2 c \sqrt{D^{2}+2 B C}\right)^{1 / 2},\left(c^{3}+4 c^{2} D\right)^{1 / 3}\right\}
\end{aligned}
$$

Since $c>\varepsilon$ was arbitrary, this concludes the proof.

Proof of Propositions 2.17 and 3.15. By the definition of $\delta_{\diamond}$, we clearly have that $\delta_{\diamond} \leq \delta_{2 \rightarrow 2}$. For signed graphexes that are $(B, C, D)$-bounded, a bound in the opposite direction follows immediately from Lemma 3.17, proving Proposition 3.15. To prove Proposition 2.17 we note that if $\delta_{\diamond}\left(\mathbb{W}_{n}, \mathbb{W}\right) \rightarrow 0$ and both $\mathbb{W}_{n}$ and $\mathbb{W}$ are (unsigned) graphexes with $D$-bounded marginals, then $\|\mathbb{W}\|_{1} \leq C$ for some $C<\infty$ by Proposition 2.4. Since $\left\|\mathbb{W}_{n}\right\|_{1}=\rho\left(\mathbb{W}_{n}\right)$ converges to $\rho(\mathbb{W})=\|W\|_{1} \leq C$ by the first statement of the lemma, we must have that $\left\|\mathbb{W}_{n}\right\|_{1} \leq \widetilde{C}$ for some $\widetilde{C}<\infty$, at which point the proof proceeds as the proof for the signed case.

Our next lemma relates the kernel norm $\|\cdot\|_{2 \rightarrow 2}$ of a two variable function $U$ to the 4-cycle counts of $U$.

Lemma 3.18. Let $U: \Omega \times \Omega \rightarrow \mathbb{R}$ be a measurable function. Then

$$
\|U\|_{2 \rightarrow 2}^{4} \leq t\left(C_{4}, U\right) \leq\|U\|_{2 \rightarrow 2}^{2}\|U\|_{2}^{2}
$$


Proof. For any $f, g$ with $\|f\|_{2}=\|g\|_{2}=1$, we have (using Cauchy's inequality)

$$
\begin{aligned}
\|f \circ U \circ g\|_{2}^{4} & \leq\|U \circ g\|_{2}^{4}=(g \circ U \circ U \circ g)^{2} \\
& =\left(\int_{\Omega^{2}} g(x) U \circ U(x, y) g(y) d \mu(x) d \mu(y)\right)^{2} \\
& \leq\left(\int_{\Omega^{2}} g(x)^{2} g(y)^{2} d \mu(x) d \mu(y)\right)\left(\int_{\Omega^{2}}(U \circ U(x, y))^{2} d \mu(x) d \mu(y)\right) \\
& =t\left(C_{4}, U\right) .
\end{aligned}
$$

This proves the first inequality. For the second, we have

$$
\begin{aligned}
t\left(C_{4}, U\right) & =\int_{\Omega^{4}} U(x, y) U(y, z) U(z, w) U(w, x) d \mu(x) d \mu(y) d \mu(z) d \mu(w) \\
& =\int_{\Omega} \int_{\Omega}\left(U \circ U_{z}\right)(x)^{2} d \mu(x) d \mu(z)=\int_{\Omega} d \mu(z)\left\|U \circ U_{z}\right\|_{2}^{2} \\
& \leq \int_{\Omega}\|U\|_{2 \rightarrow 2}^{2}\left\|U_{z}\right\|_{2}^{2}=\|U\|_{2 \rightarrow 2}^{2}\|U\|_{2}^{2} .
\end{aligned}
$$

Our next goal is to relate the jumble and the kernel distances. The next proposition shows that for $(B, C, D)$-bounded graphexes, they are equivalent. As we will see, similarly to the proof of Propositions 2.17 and 3.15, the proof also gives that for (unsigned) graphexes, the metrics $\delta_{2 \rightarrow 2}$ and $\delta_{\bigotimes}$ are equivalent on the space of graphexes with $D$-bounded marginals; see Remark 3.23 below.

Proposition 3.19. Given $B, C, D<\infty$, there exists a constant $c<\infty$ such that if $\mathbb{W}_{1}$ and $\mathbb{W}_{2}$ are $(B, C, D)$-bounded signed graphexes, then the following hold.

(1) If $d_{2 \rightarrow 2}\left(\mathbb{W}_{1}, \mathbb{W}_{2}\right) \leq \widetilde{\varepsilon}$, then $d_{\bigotimes}\left(\mathbb{W}_{1}, \mathbb{W}_{2}\right) \leq \max \left\{\widetilde{\varepsilon}, \widetilde{\varepsilon}^{3}\right\}$.

(2) If $d_{\bigotimes}\left(\mathbb{W}_{1}, \mathbb{W}_{2}\right) \leq \varepsilon$, then $d_{2 \rightarrow 2}\left(\mathbb{W}_{1}, \mathbb{W}_{2}\right) \leq \max \{\sqrt[3]{\varepsilon}, c \sqrt[4]{\varepsilon}\}$.

If the graphexes are such that $\rho\left(\mathbb{W}_{1}\right)=\rho\left(\mathbb{W}_{2}\right)$, then these bounds can be replaced by $d_{\bigotimes}\left(\mathbb{W}_{1}, \mathbb{W}_{2}\right) \leq \widetilde{\varepsilon}$ and $d_{2 \rightarrow 2}\left(\mathbb{W}_{1}, \mathbb{W}_{2}\right) \leq c \sqrt[4]{\varepsilon}$.

To prove the proposition, we establish three preliminary lemmas.

Lemma 3.20. Given a bounded, nonnegative, measurable function $f$ on some measure space $(\Omega, \mathcal{F}, \mu)$, we have

$$
\frac{\|f\|_{2}^{2}}{\sqrt{\|f\|_{1}\|f\|_{\infty}}} \leq \sup _{S \subseteq \Omega} \frac{1}{\sqrt{\mu(S)}} \int_{S} f d \mu \leq\|f\|_{2} .
$$

In particular, the second and third term define equivalent norms, for any $C, D$, on the space of nonnegative functions with $\|f\|_{1} \leq C,\|f\|_{\infty} \leq D$.

Proof. The second inequality follows from Cauchy's inequality. For the first one, let $\|f\|_{\infty}=K$. First, note that

$$
\int_{0}^{K} d c \int_{\{f \geq c\}} f d \mu=\int_{\Omega} d \mu(x) f(x) \int_{0}^{f(x)} d c=\int_{\Omega} f^{2} d \mu .
$$


We then have

$$
\begin{aligned}
\|f\|_{2}^{4} & =\left(\int_{\Omega} f^{2} d \mu\right)^{2}=\left(\int_{0}^{K} \int_{\{f \geq c\}} f d \mu d c\right)^{2} \\
& =\left(\int_{0}^{K} d c \sqrt{\mu(\{f \geq c\})} \frac{\int_{\{f \geq c\}} f d \mu}{\sqrt{\mu(\{f \geq c\})}}\right)^{2} \\
& \leq \int_{0}^{K} \frac{\left(\int_{\{f \geq c\}} f d \mu\right)^{2}}{\mu(\{f \geq c\})} d c \int_{0}^{K} \mu(\{f \geq c\}) d c .
\end{aligned}
$$

Using the fact that

we have that

$$
\int_{0}^{K} \mu(\{f \geq c\}) d c=\int_{\Omega} f d \mu
$$

$$
\int_{0}^{K} \frac{\left(\int_{\{f \geq c\}} f d \mu\right)^{2}}{\mu(\{f \geq c\})} d c \geq \frac{\|f\|_{2}^{4}}{\|f\|_{1}},
$$

which means that there exists some $c$ such that

$$
\frac{\left(\int_{\{f \geq c\}} f d \mu\right)^{2}}{\mu(\{f \geq c\})} \geq \frac{\|f\|_{2}^{4}}{\|f\|_{1} K}=\frac{\|f\|_{2}^{4}}{\|f\|_{1}\|f\|_{\infty}} .
$$

Taking $S$ to be $\{f \geq c\}$, the lemma is proved.

The following lemma is an easy corollary of Lemma 3.20 .

Lemma 3.21. Given a bounded, measurable, not necessarily nonnegative function $f$ on some measure space $(\Omega, \mu)$, we have

$$
\frac{\|f\|_{2}^{2}}{\sqrt{2\|f\|_{1}\|f\|_{\infty}}} \leq \sup _{S \subseteq \Omega} \frac{1}{\sqrt{\mu(S)}}\left|\int_{S} f d \mu\right| \leq\|f\|_{2} .
$$

Proof. The second inequality again follows from Cauchy's inequality, so we just have to prove the first one. Note that the left term is not affected by replacing $f$ with $|f|$. Let $S$ be any subset of $\Omega$, let $S^{+}$consist of the points in $S$ where $f$ is nonnegative, and let $S^{-}$be the rest. Then

$$
\begin{aligned}
\frac{1}{\sqrt{\mu(S)}} & \int_{S}|f| d \mu=\frac{\left|\int_{S^{+}} f d \mu\right|}{\sqrt{\mu\left(S^{+}\right)}} \frac{\sqrt{\mu\left(S^{+}\right)}}{\sqrt{\mu(S)}}+\frac{\left|\int_{S^{-}} f d \mu\right|}{\sqrt{\mu\left(S^{-}\right)}} \frac{\sqrt{\mu\left(S^{-}\right)}}{\sqrt{\mu(S)}} \\
& \leq \max \left(\frac{\left|\int_{S^{+}} f d \mu\right|}{\sqrt{\mu\left(S^{+}\right)}}, \frac{\left|\int_{S^{-}} f d \mu\right|}{\sqrt{\mu\left(S^{-}\right)}}\right)\left(\frac{\sqrt{\mu\left(S^{+}\right)}}{\sqrt{\mu(S)}}+\frac{\sqrt{\mu\left(S^{-}\right)}}{\sqrt{\mu(S)}}\right) \\
& \leq \max \left(\frac{\left|\int_{S^{+}} f d \mu\right|}{\sqrt{\mu\left(S^{+}\right)}}, \frac{\left|\int_{S^{-}} f d \mu\right|}{\sqrt{\mu\left(S^{-}\right)}}\right) \sqrt{2} \leq \sqrt{2} \sup _{S \subseteq \Omega} \frac{1}{\sqrt{\mu(S)}}\left|\int_{S} f d \mu\right| .
\end{aligned}
$$

Therefore, we have

$$
\sup _{S \subseteq \Omega} \frac{1}{\sqrt{\mu(S)}}\left|\int_{S} f d \mu\right| \geq \sup _{S \subseteq \Omega} \frac{1}{\sqrt{2}} \frac{1}{\sqrt{\mu(S)}} \int_{S}|f| d \mu \geq \frac{\|f\|_{2}^{2}}{\sqrt{2\|f\|_{1}\|f\|_{\infty}}} .
$$

This completes the proof. 
Lemma 3.22. For any measurable $U: \Omega \times \Omega \rightarrow \mathbb{R}$,

$$
\|U\|_{2 \rightarrow 2} \geq\|U\|_{\square} \geq \frac{\|U\|_{2 \rightarrow 2}^{4}}{8\|U\|_{\infty}^{3 / 4}\left\|D_{|U|}\right\|_{\infty}^{3 / 4}\left\|D_{|U|}\right\|_{2}^{3 / 2}} \geq \frac{\|U\|_{2 \rightarrow 2}^{4}}{8\|U\|_{\infty}^{3 / 4}\left\|D_{|U|}\right\|_{\infty}^{3 / 2}\|U\|_{1}^{3 / 4}} .
$$

Proof. Fix $f$ and $g$ with $\|f\|_{2}=\|g\|_{2}=1$ and recall that we use $U_{x}$ to denote the function $y \mapsto U(x, y)$. First, we have

$$
\|U \circ g\|_{\infty} \leq \sup _{x}\left\|U_{x}\right\|_{2} \leq \sqrt{\|U\|_{\infty}\left\|D_{|U|}\right\|_{\infty}} .
$$

We also have

$$
\begin{aligned}
\|U \circ g\|_{1} & \leq 2 \sup _{S \subseteq \Omega}\left|\int_{S} d x \int_{\Omega} U(x, y) g(y) d y\right|=2 \sup _{S \subseteq \Omega}\left|\int_{\Omega} d y g(y) \int_{S} U(x, y) d x\right| \\
& \leq 2 \int_{\Omega}|g(y)| D_{|U|}(y) \leq 2\left\|D_{|U|}\right\|_{2} .
\end{aligned}
$$

Combined with the first bound from Lemma 3.21 and the fact that $|f \circ U \circ g| \leq$ $\|U \circ g\|_{2}$, this shows that

$$
\begin{aligned}
\sup _{S \subseteq \Omega} \frac{1}{\sqrt{\mu(S)}}\left|\int_{S} U \circ g(x) d \mu(x)\right| & \geq \frac{\|U \circ g\|_{2}^{2}}{\sqrt{2\|U \circ g\|_{\infty}\|U \circ g\|_{1}}} \\
& \geq \frac{(f \circ U \circ g)^{2}}{2\|U\|_{\infty}^{1 / 4}\left\|D_{|U|}\right\|_{\infty}^{1 / 4}\left\|D_{|U|}\right\|_{2}^{1 / 2}} .
\end{aligned}
$$

Analogously, defining $g_{S}$ as the function $x \mapsto \frac{1}{\sqrt{\mu(S)}} 1_{x \in S}$, and observing that $\frac{1}{\sqrt{\mu(S)}}\left|\int_{S}(U \circ g) d \mu\right|=\left|g \circ U \circ g_{S}\right| \leq\left\|U \circ g_{S}\right\|_{2}$, we have

$$
\begin{gathered}
\sup _{T \subseteq \Omega}\left|\frac{1}{\sqrt{\mu(T) \mu(S)}} \int_{S \times T} d \mu(x) d \mu(y) U(x, y)\right| \\
\quad=\sup _{T \subseteq \Omega} \frac{1}{\sqrt{\mu(T)}}\left|\int_{T}\left(U \circ g_{S}\right)(y) d \mu(y)\right| \\
\quad \geq \frac{\left\|U \circ g_{S}\right\|_{2}^{2}}{2\|U\|_{\infty}^{1 / 4}\left\|D_{|U|}\right\|_{\infty}^{1 / 4}\left\|D_{|U|}\right\|_{2}^{1 / 2}} \\
\geq \frac{(f \circ U \circ g)^{4}}{8\|U\|_{\infty}^{3 / 4}\left\|D_{|U|}\right\|_{\infty}^{3 / 4}\left\|D_{|U|}\right\|_{2}^{3 / 2}} .
\end{gathered}
$$

Therefore,

$$
\|U\|_{\otimes} \geq \frac{\|U\|_{2 \rightarrow 2}^{4}}{8\|U\|_{\infty}^{3 / 4}\left\|D_{|U|}\right\|_{\infty}^{3 / 4}\left\|D_{|U|}\right\|_{2}^{3 / 2}} \geq \frac{\|U\|_{2 \rightarrow 2}^{4}}{8\|U\|_{\infty}^{3 / 4}\left\|D_{|U|}\right\|_{\infty}^{3 / 2}\|U\|_{1}^{3 / 4}},
$$

where in the last step we used that $\left\|D_{|U|}\right\|_{2}^{2} \leq\left\|D_{|U|}\right\|_{1}\left\|D_{|U|}\right\|_{\infty}=\|U\|_{1}\left\|D_{|U|}\right\|_{\infty}$.

Proof of Proposition 3.19. The proposition follows immediately from Lemmas 3.21 and 3.22 and the definition of the distances $d_{2 \rightarrow 2}$ and $d_{\bigotimes}$, with $c$ being the constant $c=\max \left\{\sqrt[8]{8 C D}, \sqrt[4]{64 B^{3 / 4} D^{3 / 2} C^{3 / 4}}\right\}$.

Remark 3.23. The above proof can easily be modified to see that for any $0<D<\infty$ the metrics $\delta_{2 \rightarrow 2}$ and $\delta_{\square}$ are equivalent on the space of (unsigned) graphexes with $D$ bounded marginals. Indeed, if $\mathbb{W}$ has bounded marginals, it is integrable, and if either 
$\delta_{2 \rightarrow 2}\left(\mathbb{W}_{n}, \mathbb{W}\right) \rightarrow 0$ or $\delta_{\bigotimes}\left(\mathbb{W}_{n}, \mathbb{W}\right) \rightarrow 0$, then $\left\|\mathbb{W}_{n}\right\|_{1}=\rho\left(\mathbb{W}_{n}\right) \rightarrow \rho(\mathbb{W})=\|\mathbb{W}\|_{1}$ This shows that we can assume that the sequences are $(C, D)$-bounded for some $C$, which means they are $(B, C, D)$-bounded for $B=1$.

We close this section with the (straightforward) proof that the homomorphism densities $t(F, \mathbb{W})$ indeed describe the expected number of injective homomorphisms from $F$ into $G_{T}(\mathbb{W})$.

Proposition 3.24. For any simple graph $F$ with no isolated vertices and graphex $\mathbb{W}$

$$
\mathbb{E}\left[\operatorname{inj}\left(F, G_{T}(\mathbb{W})\right)\right]=T^{|V(F)|} t(F, \mathbb{W})
$$

If the marginals of $\mathbb{W}$ are bounded, then the right side is finite, with

$$
t(F, \mathbb{W}) \leq \prod_{i}\|\mathbb{W}\|_{1}\left\|D_{\mathbb{W}}\right\|_{\infty}^{v\left(F_{i}\right)-2}
$$

where the product runs over the components of $F$ and $v_{i}$ is the number of vertices in $F_{i}$.

Proof. Recall that we extended the feature space $\Omega$ to include an additional point $\infty$, and that we labeled the vertices corresponding to the leaves of a star generated by $S$, as well as the two endpoints of the isolated edges coming from $I$, by $\infty$. Let $k=|V(F)|$. First, suppose that $\Omega$ has finite measure. Then the probability that there are $n$ points sampled from $\Omega$ is $e^{-\mu(\Omega) T} \frac{(T \mu(\Omega))^{n}}{n !}$. Let $V_{2}$ be the set of vertices of $F$ of degree at least 2 , let $V_{1}$ be the set of vertices of degree 1 whose neighbor is in $V_{2}$, and let $V_{0}$ be the set of vertices that belong to an isolated edge. Each vertex in $V_{2}$ must be mapped to a vertex with feature label in $\Omega$. Each vertex in $V_{1}$ must be mapped either to a vertex with feature label in $\Omega$ or a vertex coming from the leaves of a the star attached to such a vertex (in which case its feature label is $\infty$ ). For an isolated edge, there are three possibilities: either it is mapped to two vertices with feature label in $\Omega$, one endpoint is mapped to such a vertex and the other to a leaf of a star whose center is the first vertex, or it is mapped to an isolated edge generated by $I$ (in which case both feature labels are $\infty$ ). Let us fix for each vertex in $V_{1}$ and $V_{0}$ whether its feature label is $\infty$ or lies in $\Omega$, noting that this uniquely determines all the choices we just discussed. Let $V_{0}^{\prime} \subseteq V_{0}$ and $V_{1}^{\prime} \subseteq V_{1}$ be the sets of vertices mapped to a vertex coming from $\Omega$, and let $V^{\prime}=V_{0}^{\prime} \cup V_{1}^{\prime} \cup V_{2}$. Let $U$ be the set of remaining vertices. Let $J$ be the set of isolated edges, and $J^{\prime \prime}$ the set of isolated edges where we have fixed that they are mapped to an edge generated by $I$; i.e., they have both endpoints in $U$. For a vertex $i \in V^{\prime}$, let $d_{U}(i)$ be its degree to 
$U$. Conditioned on $V^{\prime}$, we have

$$
\begin{aligned}
& \mathbb{E}\left[\operatorname{inj}\left(F, G_{T}\right) \mid V^{\prime}\right]=\sum_{n=0}^{\infty} e^{-\mu(\Omega) T} \frac{(T \mu(\Omega))^{n}}{n !} \frac{(n)_{\left|V^{\prime}\right|}}{\mu(\Omega)^{\left|V^{\prime}\right|}} \\
& \int_{\Omega^{V^{\prime}}} \prod_{\{i, j\} \in E\left(\left.F\right|_{V^{\prime}}\right)} W\left(x_{i}, x_{j}\right) \prod_{i \in V^{\prime}}\left(T S\left(x_{i}\right)\right)^{d_{U}(i)}\left(2 T^{2} I\right)^{\left|J^{\prime \prime}\right|} \\
& =\sum_{n=\left|V^{\prime}\right|}^{\infty} T^{\left|V^{\prime}\right|} e^{-\mu(\Omega) T} \frac{(T \mu(\Omega))^{n-\left|V^{\prime}\right|}}{\left(n-\left|V^{\prime}\right|\right) !} \\
& \int_{\Omega^{V^{\prime}}} T^{|U|} \prod_{\{i, j\} \in E\left(\left.F\right|_{V^{\prime}}\right)} W\left(x_{i}, x_{j}\right) \prod_{i \in V^{\prime}}\left(S\left(x_{i}\right)\right)^{d_{U}(i)}(2 I)^{\left|J^{\prime \prime}\right|} \\
& =T^{|V|}\left(\sum_{n=\left|V^{\prime}\right|}^{\infty} e^{-\mu(\Omega) T} \frac{(T \mu(\Omega))^{n-\left|V^{\prime}\right|}}{\left(n-\left|V^{\prime}\right|\right) !}\right) \\
& \left(\int_{\Omega^{V^{\prime}}} \prod_{\{i, j\} \in E\left(\left.F\right|_{V^{\prime}}\right)} W\left(x_{i}, x_{j}\right) \prod_{i \in V^{\prime}}\left(S\left(x_{i}\right)\right)^{d_{U}(i)}(2 I)^{\left|J^{\prime \prime}\right|}\right) \\
& =T^{|V|} \int_{\Omega^{V^{\prime}}} \prod_{\{i, j\} \in E\left(\left.F\right|_{V^{\prime}}\right)} W\left(x_{i}, x_{j}\right) \prod_{i \in V^{\prime}}\left(S\left(x_{i}\right)\right)^{d_{U}(i)}(2 I)^{\left|J^{\prime \prime}\right|} \text {. }
\end{aligned}
$$

Therefore,

$$
\mathbb{E}\left[\operatorname{inj}\left(F, G_{T}\right)\right]=T^{|V|} \sum_{\substack{V_{0}^{\prime} \subseteq V_{0} \\ V_{1}^{\prime} \subseteq V_{1}}} \int_{\Omega^{V^{\prime}}} \prod_{\{i, j\} \in E\left(\left.F\right|_{V^{\prime}}\right)} W\left(x_{i}, x_{j}\right) \prod_{i \in V^{\prime}}\left(S\left(x_{i}\right)\right)^{d_{U}(i)}(2 I)^{\left|J^{\prime \prime}\right|} .
$$

Now, it is not difficult to check that this is multiplicative over connected components of $F$. Indeed, each term with fixed $V_{0}^{\prime}, V_{1}^{\prime}$ is multiplicative, and the choice of which vertices to put in $V_{0}^{\prime}, V_{1}^{\prime}$ from each of the components is independent. Therefore, we may assume that $F$ is connected.

If $F$ consists of a single edge $\{i, j\}$, then the above expression gives

$$
\mathbb{E}\left[\operatorname{inj}\left(F, G_{T}\right)\right]=T^{2}\left(\int_{\Omega^{2}} W(x, y) d x d y+2 \int_{\Omega} S(x) d x+2 I\right)=T^{2} t(F, \mathbb{W}),
$$


as required. Otherwise, $F$ has no isolated edges, so $V_{0}$ is empty (and so is $J^{\prime \prime}$ ). We then have

$$
\begin{aligned}
\mathbb{E}\left[\operatorname{inj}\left(F, G_{T}\right)\right] & =T^{|V|} \sum_{V_{1}^{\prime} \subseteq V_{1}} \int_{\Omega^{V^{\prime}}} \prod_{\{i, j\} \in E\left(\left.F\right|_{V^{\prime}}\right)} W\left(x_{i}, x_{j}\right) \prod_{i \in V_{2}}\left(S\left(x_{i}\right)\right)^{d_{U}(i)} \\
& =T^{|V|} \int_{\Omega^{V_{2}}} \prod_{\{i, j\} \in E\left(F_{V_{2}}\right)} W\left(x_{i}, x_{j}\right) \cdot \\
& \prod_{i \in V_{2}}\left(\sum_{T_{i} \subseteq N_{V_{1}}(i)} \int_{\Omega^{T_{i}}} \prod_{j \in T_{i}} W\left(x_{i}, x_{j}\right) S\left(x_{i}\right)^{\left|N_{V_{1}}(i)\right|-\left|T_{i}\right|}\right) \\
& \prod_{\Omega^{V_{2}}} \prod_{\{i, j\} \in E\left(F_{V_{2}}\right)} W\left(x_{i}, x_{j}\right) \cdot \\
& \prod_{i \in V_{2}}\left(\int_{\Omega} W\left(x_{i}, x_{j}\right) d x_{j}+S\left(x_{i}\right)\right)^{\left|N_{V_{1}}(i)\right|} \\
& =T^{|V|} t(F, \mathbb{W}) .
\end{aligned}
$$

This completes the proof of the first statement if $\Omega$ has finite measure. The general $\sigma$-finite case follows by monotone convergence, with both sides being possibly infinite in the limit.

To prove the second statement we consider the components of $F$ separately. Furthermore, given a component $F^{\prime}$ of $F$, we use the fact that $\|W\|_{\infty} \leq 1$ to delete edges from $F^{\prime}$ until $F^{\prime}$ becomes a tree. At this point, we can remove the leaves of the tree at the cost of a factor $D$ for each leaf, getting a new tree with less edges. We continue until we are left with a single edge, at which point we bound the remaining integral by $\|\mathbb{W}\|_{1}$.

\section{Tightness}

The goal of this section is to establish various equivalent notions of tightness, and to then use tightness to relate convergence in the kernel and the weak kernel metric. In particular, we will relate the convergence of a sequence of graphexes in the weak kernel metric $\delta_{\diamond}$ to convergence of a "regularized" sequence in the kernel metric $\delta_{2 \rightarrow 2}$, where the regularized sequence is obtained from the original one by discarding the part of the space which has large marginals; see Proposition 4.6 below. In contrast to the last section, in this section we restrict ourselves to unsigned graphexes since we believe that the obvious generalization of the notion of tightness to signed graphexes will not be the right notion of tightness for the metric $\delta_{\diamond}$; see Remark 4.8 at the end of this section.

We start by establishing the equivalence of various formulations of tightness.

Theorem 4.1. Given a set of graphexes $\mathcal{S}$, the following are equivalent:

(1) $\mathcal{S}$ is tight. In other words, for every $\varepsilon>0$, there exist $C$ and $D$ such that for every graphex $\mathbb{W} \in \mathcal{S}, \mathbb{W}=(W, S, I, \boldsymbol{\Omega})$ with $\boldsymbol{\Omega}=(\Omega, \mathcal{F}, \mu)$, there exists $\Omega_{\varepsilon} \subseteq \Omega$ such that $\mu\left(\Omega_{\varepsilon}\right) \leq \varepsilon$ and $\mathbb{W}^{\prime}=\left.\mathbb{W}\right|_{\Omega \backslash \Omega_{\varepsilon}}$ has $\left\|\mathbb{W}^{\prime}\right\|_{1} \leq C$, $\left\|D_{\mathbb{W}^{\prime}}\right\|_{\infty} \leq D$. 
(2) For every $\varepsilon>0$, there exist $C$ such that for every graphex $\mathbb{W} \in \mathcal{S}, \mathbb{W}=$ $(W, S, I, \Omega)$, there exists $\Omega_{\varepsilon} \subseteq \Omega$ such that $\mu\left(\Omega_{\varepsilon}\right) \leq \varepsilon$ and $\mathbb{W}^{\prime}=\left.\mathbb{W}\right|_{\Omega \backslash \Omega_{\varepsilon}}$ has $\left\|\mathbb{W}^{\prime}\right\|_{1} \leq C$.

(3) For every $\varepsilon$, there is a $D$ and $C$ such that for any $\mathbb{W} \in \mathcal{S}$, taking $\Omega_{\leq D}$ to be the set of points with $D_{\mathbb{W}}(x) \leq D, \mu\left(\Omega \backslash \Omega_{\leq D}\right) \leq \varepsilon$, and $\left\|\left.\mathbb{W}\right|_{\Omega_{\leq D}}\right\|_{1} \leq C$.

(4) For every $T>0$, the set of random unlabeled finite graphs $G_{T}(\mathbb{W})$ with $\mathbb{W} \in \mathcal{S}$ is tight.

(5) There exists $T>0$ such that the set of random unlabeled finite graphs $G_{T}(\mathbb{W})$ with $\mathbb{W} \in \mathcal{S}$ is tight.

Corollary 4.2. Let $\mathcal{S}$ be a set of graphexes.

(1) If there exists a $C<\infty$ such that $\|\mathbb{W}\|_{1} \leq C$ for all $\mathbb{W} \in \mathcal{S}$, then $\mathcal{S}$ is tight.

(2) If $\mathcal{S}$ is tight and has uniformly bounded marginals, then there exist $C, D<\infty$ such that $\mathcal{S}$ is $(C, D)$-bounded.

Proof. (1) Taking $\Omega_{\varepsilon}=\emptyset$ for any $\varepsilon$, the set $\mathcal{S}$ clearly satisfies condition (2) from the theorem.

(2) Choose $\varepsilon$ arbitrarily, say $\varepsilon=1$, and let $C^{\prime}, D^{\prime}$ be such that (3) from Theorem 4.1 holds. Furthermore, let $D$ be such that the marginals of all graphexes in $\mathcal{S}$ are bounded by $D$. Then

$$
\begin{aligned}
\|\mathbb{W}\|_{1} & \leq\left\|\left.\mathbb{W}\right|_{\Omega_{\leq D^{\prime}}}\right\|_{1}+2 \int W(x, y) 1_{D_{\mathbb{W}}(x)>D^{\prime}} d \mu(x) d \mu(y)+2 \int_{D_{\mathbb{W}}>D^{\prime}} S(x) d \mu(x) \\
& \leq C^{\prime}+2 \int_{D_{\mathbb{W}}>D^{\prime}} D_{\mathbb{W}(x)} d \mu(x) \leq C^{\prime}+2 D \varepsilon=C^{\prime}+2 D=: C
\end{aligned}
$$

proving the claim.

In order to prove Theorem 4.1, we will use the following lemma.

Lemma 4.3. The probability that $G_{T}(\mathbb{W})$ has more than $K T^{2}\|\mathbb{W}\|_{1}$ edges is at most

$$
\frac{T^{2}\|\mathbb{W}\|_{1} / 2+T^{3}\left\|D_{\mathbb{W}}\right\|_{2}^{2}}{(K-1 / 2)^{2} T^{4}\|\mathbb{W}\|_{1}^{2}}
$$

and the probability that it has less than $T^{2}\|\mathbb{W}\|_{1} / 4$ edges is at most

$$
\frac{16\left(T^{2}\|\mathbb{W}\|_{1} / 2+T^{3}\left\|D_{\mathbb{W}}\right\|_{2}^{2}\right)}{T^{4}\|\mathbb{W}\|_{1}^{2}}
$$

Proof. Let $X_{T}$ be the number of edges of $G_{T}(\mathbb{W})$. By Proposition $3.24, X_{T}$ has expectation $T^{2}\|\mathbb{W}\|_{1} / 2$. To calculate the variance, note that we have

$$
X_{T}^{2}=\frac{\operatorname{inj}\left(F_{1}, G_{T}\right)}{2}+\frac{\operatorname{inj}\left(F_{2}, G_{T}\right)}{4}+\operatorname{inj}\left(F_{3}, G_{T}\right)
$$

where $F_{1}$ consists of a single edge, $F_{2}$ consists of a pair of disjoint edges, and $F_{3}$ consists of two edges joined at one vertex. Therefore, we can again use Proposition 3.24 to conclude that

$$
\begin{aligned}
\operatorname{Var}\left(X_{T}\right) & =\mathbb{E}\left[X_{T}^{2}\right]-\mathbb{E}\left[X_{T}\right]^{2} \\
& =\frac{T^{2}\|\mathbb{W}\|_{1}}{2}+\frac{T^{4}\left\|\mathbb{W}_{1}\right\|^{2}}{4}+T^{3}\left\|D_{\mathbb{W}}\right\|_{2}^{2}-\left(\frac{T^{2}\|\mathbb{W}\|_{1}}{2}\right)^{2} \\
& =\frac{T^{2}\|\mathbb{W}\|_{1}}{2}+T^{3}\left\|D_{\mathbb{W}}\right\|_{2}^{2}
\end{aligned}
$$


The bounds on the probabilities of having too many or too few edges follow from Chebyshev's inequality.

Proof of Theorem 4.1. (1) $\Rightarrow(2)$ is obvious.

$(2) \Rightarrow(1)$ : Suppose $\mathcal{S}$ satisfies (2), and let $\varepsilon>0$. Take $C$ from property (2) for $\varepsilon / 2$, and take $D=2 C / \varepsilon$. For each $\mathbb{W} \in \mathcal{S}$ with underlying space $\Omega$, there is a set $\Omega^{\prime} \subseteq \Omega$ with $\mu\left(\Omega \backslash \Omega^{\prime}\right) \leq \varepsilon / 2$ so that the restriction $\mathbb{W}^{\prime}=\left.\mathbb{W}\right|_{\Omega}$ has $\left\|\mathbb{W}^{\prime}\right\|_{1} \leq C$. Suppose $\mu\left(x \in \Omega^{\prime}: D_{\mathbb{W}^{\prime}}(x)>D\right)>\varepsilon / 2$. Then we would have $\left\|W^{\prime}\right\|_{1}>D \varepsilon / 2=C$, a contradiction. Therefore, removing the set of points with $D_{\mathbb{W}^{\prime}}(x)>D$, we have removed points with total measure at most $\varepsilon$, and the restricted graphex is $(C, D)$-bounded.

$(3) \Rightarrow(1)$ is obvious.

$(1) \Rightarrow(4)$ : Fix $T>0$ and $\varepsilon>0$. Take $\varepsilon^{\prime}$ such that $e^{-T \varepsilon^{\prime}}>1-\varepsilon / 2$, and take $C, D$ for $\mathcal{S}$ from the definition of tightness. Given $\mathbb{W} \in \mathcal{S}$, there exists $\Omega_{\varepsilon^{\prime}} \subseteq \Omega$ such that $\mu\left(\Omega_{\varepsilon^{\prime}}\right) \leq \varepsilon^{\prime}$ and $\mathbb{W}^{\prime}=\left.\mathbb{W}\right|_{\Omega \backslash \Omega_{\varepsilon^{\prime}}}$ has $\left\|\mathbb{W}^{\prime}\right\|_{1} \leq C,\left\|D_{\mathbb{W}^{\prime}}\right\|_{\infty} \leq D$. The probability that $G_{T}(\mathbb{W})$ samples a point in $\Omega \backslash \Omega_{\varepsilon^{\prime}}$ during the Poisson process is at most $1-e^{-T \varepsilon^{\prime}}<\varepsilon / 2$. Conditioned on this not happening, the sample is equivalent to a sample from $G_{T}\left(\mathbb{W}^{\prime}\right)$. For this, we have that $\left\|D_{\mathbb{W}^{\prime}}\right\|_{2}^{2} \leq C D$; therefore we can take $K=K(C, D)$ large enough so that the probability that there are more than $K T^{2} C$ edges in $G_{T}\left(\mathbb{W}^{\prime}\right)$ is at most $\varepsilon / 2$ (independently of $\mathbb{W}^{\prime}$ ). Therefore, the probability that there are more than $K T^{2} C$ edges in $G_{T}(\mathbb{W})$ is at most $\varepsilon$.

$(4) \Rightarrow(5)$ is obvious.

$(5) \Rightarrow(3)$ : Let $\varepsilon>0$. First, we show that there exists a $D$ so that for every $\mathbb{W} \in \mathcal{S}$, the measure of the set $\left\{D_{\mathbb{W}}>D\right\}$ is at most $\varepsilon$. Suppose not. We will show that this implies that for each $M$ we can find a $\mathbb{W} \in \mathcal{S}$ such with probability at least $\frac{1}{2}\left(1-e^{-\varepsilon T / 2}\right)$, the number of edges in $G_{T}(\mathbb{W})$ is at least $M$. This contradicts the assumption that the set of random graphs $G_{T}(\mathbb{W})$ is tight.

Assume thus that for every $D$, there exists a $\mathbb{W}=\mathbb{W}(D) \in \mathcal{S}$ such that the set $\left\{D_{\mathbb{W}}>D\right\}$ has measure larger than $\varepsilon$. Take $G_{T}(\mathbb{W})$ and randomly color the vertices red and blue. With probability at least $1-e^{-\varepsilon T / 2}$, there exists at least one blue point whose feature label falls into the set $\left\{D_{\mathbb{W}}>D\right\}$. Conditioned on this, taking a blue point with feature label $x \in\left\{D_{\mathbb{W}}>D\right\}$, the number of red neighbors it has is a Poisson random variable with mean $T D_{\mathbb{W}}(x) / 2$. Given $M<\infty$, choose $D=D(M, T)$ in such a way that a Poisson random variable with mean at least $T D / 2$ has probability at least $1 / 2$ of being greater than $M$. As a consequence, given $T$ and an arbitrary large $M$ and we can find a $D$ and $\mathbb{W}=\mathbb{W}(D) \in \mathcal{S}$ such that with probability at least $\frac{1}{2}\left(1-e^{-\varepsilon T / 2}\right)$, the number of edges in $G_{T}(\mathbb{W})$ is at least $M$, contradicting tightness.

We claim that $\left\|\left.\mathbb{W}\right|_{\Omega_{\leq D}}\right\|_{1}$ can't be arbitrarily large. Set $\mathbb{W}^{\prime}=\left.\mathbb{W}\right|_{\Omega_{\leq D}}$ and assume that $\left\|\mathbb{W}^{\prime}\right\|_{1}=C$. Then the probability that $G_{T}\left(\mathbb{W}^{\prime}\right)$ has less than $T^{2} C / 4$ edges is at most

$$
\frac{8+16 T D}{T^{2} C} \text {. }
$$

If $C$ is large enough, this is less than $1 / 2$. But then for large enough $C$ with probability at least $1 / 2$, the number of edges is at least $T^{2} C / 4$, contradicting the assumption of tightness. This means that $C$ can't be arbitrarily large.

This completes the proof of the theorem. 
Remark 4.4. It will sometimes be useful to transform a graphex $\mathbb{W}$ over an arbitrary $\sigma$-finite space $\boldsymbol{\Omega}=(\Omega, \mathcal{F}, \mu)$ into a graphex over an atomless space by mapping $\boldsymbol{\Omega}$ to the product space $\Omega \times[0,1]$ equipped with the measure $\mu \times \lambda$, with $\lambda$ denoting the Lebesgue measure, and mapping $\mathbb{W}$ to $\Phi(\mathbb{W})=\mathbb{W}^{\phi}$, with $\phi: \Omega \times[0,1] \rightarrow \Omega$ denoting the coordinate projection onto $\Omega$. It is easy to see that $\delta_{\diamond}(\mathbb{W}, \Phi(\mathbb{W}))=0$, which together with the triangle inequality implies that the map $\Phi$ does not change distances between graphons. It is also easy to check that if $\mathcal{S}$ is a tight set of graphexes, then the set of graphexes obtained by mapping each graphex $\mathbb{W} \in \mathcal{S}$ to the corresponding atomless graphex $\Phi(\mathbb{W})$ is tight as well.

Let us analyze when graphexes converge under $\delta_{\diamond}$. To this end, we first prove a few lemmas.

Lemma 4.5. Given $C, D, M \in(0, \infty)$, there exists a function $f:[0, \infty)^{2} \rightarrow[0, \infty)$ such that $f(x) \rightarrow 0$ as $x \rightarrow 0$ and such that the following holds

(1) Let $\mathbb{W}$ be a graphex over $(\Omega, \mathcal{F}, \mu)$, and let $\widetilde{\mu}$ be a second measure over $(\Omega, \mathcal{F})$ such that $\mu-r \leq \widetilde{\mu} \leq \mu$. If $\widetilde{\mathbb{W}}$ is obtained from $\mathbb{W}$ by replacing $\mu$ with $\widetilde{\mu}$ then

$$
\mu\left(D_{\mathbb{W}}>D+r\right)-r \leq \widetilde{\mu}\left(D_{\widetilde{\mathbb{W}}}>D\right) \leq \mu\left(D_{\mathbb{W}}>D\right)
$$

for all $D>0$.

(2) Let $\mathbb{W}_{1}$ and $\mathbb{W}_{2}$ be graphexes with bounded marginals, defined over the same space $(\Omega, \mathcal{F}, \mu)$. Suppose that $d_{2 \rightarrow 2}\left(\mathbb{W}_{1}, \mathbb{W}_{2}\right)<\varepsilon$. Then

$$
\mu\left(\left\{\left|D_{\mathbb{W}_{1}}-D_{\mathbb{W}_{2}}\right| \geq \varepsilon\right\}\right)<\varepsilon^{2} .
$$

(3) Let $\widetilde{\mathbb{W}}_{1}$ and $\widetilde{\mathbb{W}}_{2}$ be graphexes with bounded marginals, defined over $\left(\Omega_{1}, \mathcal{F}_{1}, \widetilde{\mu}_{1}\right)$ and $\left(\Omega_{2}, \mathcal{F}_{2}, \widetilde{\mu}_{2}\right)$. If $\delta_{2 \rightarrow 2}\left(\widetilde{\mathbb{W}}_{1}, \widetilde{\mathbb{W}}_{2}\right)<\varepsilon$ and $D>\varepsilon$, then

$$
\widetilde{\mu}_{1}\left(\left\{D_{\widetilde{\mathbb{W}}_{1}}>D+\varepsilon\right\}\right)-\varepsilon^{2} \leq \widetilde{\mu}_{2}\left(\left\{D_{\widetilde{\mathbb{W}}_{2}}>D\right\}\right) \leq \widetilde{\mu}_{1}\left(\left\{D_{\widetilde{\mathbb{W}}_{1}}>D-\varepsilon\right\}\right)+\varepsilon^{2} .
$$

(4) For $i=1,2$, let $\mathbb{W}_{i}$ be graphexes defined over $\left(\Omega_{i}, \mathcal{F}_{i}, \mu_{i}\right)$, and let $\mathbb{W}_{i, \leq D}$ be the restriction of $\mathbb{W}_{i}$ to the subset $\left\{D_{\mathbb{W}_{i}} \leq D\right\}$ of $\Omega_{i}$. Assume that $\varepsilon+\varepsilon^{2}<D, \mu_{1}\left(\left\{D_{\mathbb{W}_{1}}>D\right\}\right)+\mu_{2}\left(\left\{D_{\mathbb{W}_{2}}>D\right\}\right) \leq M,\left\|\mathbb{W}_{2, \leq D}\right\|_{1} \leq C$, $\delta_{\diamond}\left(\mathbb{W}_{1}, \mathbb{W}_{2}\right) \leq \varepsilon$, and $\mu_{2}\left(\left\{D-\varepsilon-\varepsilon^{2}<D_{\mathbb{W}_{2}} \leq D+\varepsilon+\varepsilon^{2}\right\}\right) \leq \bar{\delta}$. Then

$$
\delta_{2 \rightarrow 2}\left(\mathbb{W}_{1, \leq D}, \mathbb{W}_{2, \leq D}\right) \leq f(\varepsilon, \delta) \text {. }
$$

Proof. (1) The assumption $\mu-r \leq \widetilde{\mu} \leq \mu$ clearly implies that for all $x \in \Omega$,

$$
D_{\mathbb{W}}(x)-r \leq D_{\widetilde{\mathbb{W}}}(x) \leq D_{\mathbb{W}}(x) .
$$

As a consequence,

$$
\mu\left(D_{\mathbb{W}}>D+r\right)-r \leq \widetilde{\mu}\left(D_{\mathbb{W}}>D+r\right) \leq \widetilde{\mu}\left(D_{\widetilde{\mathbb{W}}}>D\right)
$$

and

$$
\widetilde{\mu}\left(D_{\widetilde{\mathbb{W}}}>D\right) \leq \widetilde{\mu}\left(D_{\mathbb{W}}>D\right) \leq \mu\left(D_{\mathbb{W}}>D\right) .
$$

(2) By the definition of $d_{2 \rightarrow 2},\left\|D_{\mathbb{W}_{1}}-D_{\mathbb{W}_{2}}\right\|_{2}^{2}<\varepsilon^{4}$, which clearly implies that

$$
\mu\left(\left\{\left|D_{\mathbb{W}_{1}}-D_{\mathbb{W}_{2}}\right| \geq \varepsilon\right\}\right)<\varepsilon^{2} .
$$

(3) For $i=1,2$, let $\left(\widetilde{\Omega}_{i}^{\prime}, \widetilde{\mathcal{F}}_{i}^{\prime}, \widetilde{\mu}_{i}^{\prime}\right)$ be a measure space obtained from $\left(\Omega_{i}, \mathcal{F}_{i}, \widetilde{\mu}_{i}\right)$ by appending some space of infinite total measure, and let $\widetilde{\mathbb{W}}_{i}^{\prime}$ be the trivial extension 
of $\widetilde{\mathbb{W}}_{i}$ onto $\left(\widetilde{\Omega}_{i}^{\prime}, \widetilde{\mathcal{F}}_{i}^{\prime}, \widetilde{\mu}_{i}^{\prime}\right)$. Furthermore, let $\mu^{\prime}$ be a coupling of $\widetilde{\mu}_{1}^{\prime}$ and $\widetilde{\mu}_{2}^{\prime}$ such that $d_{2 \rightarrow 2}\left(\mathbb{W}_{1}^{\prime}, \mathbb{W}_{2}^{\prime}\right) \leq \varepsilon$, where $\mathbb{W}_{i}^{\prime}=\left(\widetilde{\mathbb{W}}_{i}^{\prime}\right)^{\pi_{i}, \mu^{\prime}}$ for $i=1,2$. Then by $(2)$,

$$
\begin{aligned}
\widetilde{\mu}_{1}\left(\left\{D_{\widetilde{\mathbb{W}}_{1}}>D+\varepsilon\right\}\right) & =\widetilde{\mu}_{1}^{\prime}\left(\left\{D_{\widetilde{\mathbb{W}}_{1}^{\prime}}>D+\varepsilon\right\}\right) \\
& =\mu^{\prime}\left(\left\{D_{\mathbb{W}_{1}^{\prime}}>D+\varepsilon\right\}\right) \\
& \leq \mu^{\prime}\left(\left\{D_{\mathbb{W}_{2}^{\prime}}>D\right\}\right)+\mu^{\prime}\left(\left\{\left|D_{\mathbb{W}_{1}^{\prime}}-D_{\mathbb{W}_{2}^{\prime}}\right| \geq \varepsilon\right\}\right) \\
& \leq \mu^{\prime}\left(\left\{D_{\mathbb{W}_{2}^{\prime}}>D\right\}\right)+\varepsilon^{2} \\
& =\widetilde{\mu}_{2}\left(\left\{D_{\mathbb{W}_{2}}>D\right\}\right)+\varepsilon^{2},
\end{aligned}
$$

proving the first bound in (2). The second is proved analogously.

(4) For $i=1,2$, let $\mathbb{W}_{i}^{\prime}$ be the trivial extension of $\mathbb{W}_{i}$ to a space $\left(\Omega_{i}^{\prime}, \mathcal{F}_{i}^{\prime}, \mu_{i}^{\prime}\right)$ obtained from $\left(\Omega_{i}, \mathcal{F}_{i}, \mu_{i}\right)$ by appending some $\sigma$-finite space of infinite total mass. Recalling Lemma 3.14, we can use the assumption $\delta_{\diamond}\left(\mathbb{W}_{1}, \mathbb{W}_{2}\right)<\varepsilon$ to infer the existence of a measure $\mu^{\prime}$ over $\Omega_{1}^{\prime} \times \Omega_{2}^{\prime}$ such that $d_{2 \rightarrow 2}\left(\left(\mathbb{W}_{1}^{\prime}\right)^{\pi_{1}, \mu^{\prime}},\left(\mathbb{W}_{2}^{\prime}\right)^{\pi_{2}, \mu^{\prime}}\right)<\varepsilon$ and $\mu_{i}^{\prime}-\varepsilon^{2} \leq\left(\mu^{\prime}\right)^{\pi_{i}} \leq \mu_{i}^{\prime}, i=1,2$. For $i=1,2$, define $\widetilde{\mu}_{i}=\left(\mu^{\prime}\right)^{\pi_{i}}, \mathbb{U}_{i}^{\prime}=\left(\mathbb{W}_{i}^{\prime}\right)^{\pi_{i}, \mu^{\prime}}$, and $\Omega_{i, \leq D}^{\prime}=\left\{x \in \Omega_{i}^{\prime}: D_{\mathbb{W}_{i}^{\prime}}(x) \leq D\right\}$. Then $\mathbb{W}_{1, \leq D}^{\prime}:=\left.\left(\mathbb{W}_{1}^{\prime}\right)\right|_{\Omega_{1, \leq D}^{\prime}}$ and $\mathbb{W}_{2, \leq D}^{\prime}:=$ $\left.\left(\mathbb{W}_{2}^{\prime}\right)\right|_{\Omega_{2, \leq D}^{\prime}}$ are extensions of $\mathbb{W}_{1, \leq D}$ and $\mathbb{W}_{2, \leq D}$ by spaces of infinite measure. Let $\mu_{1, D}^{\prime}$ and $\mu_{2, D}^{\prime}$ be the marginals of the measure $\left.\mu^{\prime}\right|_{\Omega_{1, \leq D}^{\prime} \times \Omega_{2, \leq D}^{\prime}}$. We then have that $\mu_{1, D}^{\prime} \leq \mu_{1}^{\prime}$. Observing that $D_{\mathbb{W}_{i}^{\prime}}\left(\pi_{i}(x)\right)-\varepsilon^{2} \leq D_{\mathbb{U}_{i}^{\prime}}(x) \leq D_{\mathbb{W}_{i}^{\prime}}\left(\pi_{i}(x)\right)$, we furthermore have that

$$
\begin{aligned}
0 & \leq\left(\mu_{1}^{\prime}-\mu_{1, D}^{\prime}\right)\left(\Omega_{1, \leq D}^{\prime}\right) \leq \varepsilon^{2}+\left(\widetilde{\mu}_{1}^{\prime}-\mu_{1, D}^{\prime}\right)\left(\Omega_{1, \leq D}^{\prime}\right) \\
& =\varepsilon^{2}+\mu^{\prime}\left(\pi_{1}^{-1}\left(\left\{D_{\mathbb{W}_{1}^{\prime}} \leq D\right\}\right) \times \pi_{2}^{-1}\left(\left\{D_{\mathbb{W}_{2}^{\prime}}>D\right\}\right)\right) \\
& \leq \varepsilon^{2}+\mu^{\prime}\left(\left\{D_{\mathbb{U}_{1}^{\prime}} \leq D\right\} \cap\left\{D_{\mathbb{U}_{2}^{\prime}}>D-\varepsilon^{2}\right\}\right) \\
& \leq 2 \varepsilon^{2}+\mu^{\prime}\left(\left\{D-\varepsilon^{2}<D_{\mathbb{U}_{2}^{\prime}} \leq D+\varepsilon\right\}\right) \\
& \leq 2 \varepsilon^{2}+\mu_{2}\left(\left\{D-\varepsilon^{2}<D_{\mathbb{W}_{2}} \leq D+\varepsilon+\varepsilon^{2}\right\}\right) \leq 2 \varepsilon^{2}+\delta=: \widetilde{\delta} .
\end{aligned}
$$

Here we used the fact that $d_{2 \rightarrow 2}\left(\mathbb{U}_{1}^{\prime}, \mathbb{U}_{2}^{\prime}\right)<\varepsilon$, which meant that we could apply (2). Similarly, $\mu_{2, D}^{\prime} \leq \mu_{2}^{\prime}$ and

$$
\begin{aligned}
0 & \leq\left(\mu_{2}^{\prime}-\mu_{2, D}^{\prime}\right)\left(\widetilde{\Omega}_{2, \leq D}^{\prime}\right) \\
& \leq \varepsilon^{2}+\mu^{\prime}\left(\left\{D_{\mathbb{U}_{1}^{\prime}}>D-\varepsilon^{2}\right\} \cap\left\{D_{\mathbb{U}_{2}^{\prime}} \leq D\right\}\right) \\
& \leq 2 \varepsilon^{2}+\mu^{\prime}\left(\left\{D-\varepsilon-\varepsilon^{2}<D_{\mathbb{U}_{2}^{\prime}} \leq D\right\}\right) \\
& \leq 2 \varepsilon^{2}+\mu_{2}\left(\left\{D-\varepsilon-\varepsilon^{2}<D_{\mathbb{W}_{2}} \leq D+\varepsilon^{2}\right\}\right) \leq \widetilde{\delta} .
\end{aligned}
$$

Next we claim that we may assume without loss of generality that

$$
\left(\mu_{1}^{\prime}-\mu_{1, D}^{\prime}\right)\left(\Omega_{1, \leq D}\right)=\left(\mu_{2}^{\prime}-\mu_{2, D}^{\prime}\right)\left(\Omega_{2, \leq D}^{\prime}\right) \leq \widetilde{\delta} .
$$

Indeed, we can trivially extend either $\mathbb{W}_{1}^{\prime}$ or $\mathbb{W}_{2}^{\prime}$ by appending a space of total measure $\delta^{\prime} \leq \widetilde{\delta}$ (e.g., the interval $\left[0, \delta^{\prime}\right)$ ), setting $\mu^{\prime}$ to zero on the additional set. This corresponds to trivially extending both $\mathbb{U}_{1}^{\prime}$ and $\mathbb{U}_{2}^{\prime}$ by either $\left[0, \delta^{\prime}\right] \times \Omega_{2}^{\prime}$ or $\Omega_{1}^{\prime} \times\left[0, \delta^{\prime}\right]$. Since $\mu^{\prime}=0$ on the extension, this does not change $d_{2 \rightarrow 2}\left(\mathbb{U}_{1}^{\prime}, \mathbb{U}_{2}^{\prime}\right)$.

Note also that

$$
\begin{aligned}
\mu^{\prime}\left(\Omega_{1}^{\prime} \times \Omega_{2}^{\prime} \backslash \Omega_{1, \leq D}^{\prime} \times \Omega_{2, \leq D}^{\prime}\right) & \leq \mu^{\prime}\left(\left\{D_{\mathbb{W}_{1}^{\prime}}>D\right\} \times \Omega_{2}^{\prime}\right)+\mu^{\prime}\left(\Omega_{1}^{\prime} \times\left\{D_{\mathbb{W}_{2}^{\prime}}>D\right\}\right) \\
& \leq \mu_{1}\left(\left\{D_{\mathbb{W}_{1}}>D\right\}\right)+\mu_{2}\left(\left\{D_{\mathbb{W}_{2}}>D\right\}\right) \leq M .
\end{aligned}
$$


If $\mathbb{U}_{1}^{\prime \prime}$ and $\mathbb{U}_{2}^{\prime \prime}$ are the restrictions of $\mathbb{U}_{1}^{\prime}$ and $\mathbb{U}_{2}^{\prime}$ to $\Omega_{1, \leq D}^{\prime} \times \Omega_{2, \leq D}^{\prime}$, and $\mu^{\prime \prime}$ is the restriction of $\mu^{\prime}$, then by Lemma 3.13 ,

$$
\begin{gathered}
\left\|\mathbb{U}_{1}^{\prime \prime}-\mathbb{U}_{2}^{\prime \prime}\right\|_{2 \rightarrow 2, \mu^{\prime \prime}} \leq \varepsilon, \\
\left\|D_{\mathbb{U}_{1}^{\prime \prime}}-D_{\mathbb{U}_{2}^{\prime \prime}}\right\|_{2, \mu^{\prime \prime}} \leq \varepsilon^{2}+\sqrt{M} \varepsilon,
\end{gathered}
$$

and

$$
\left|\left\|\mathbb{U}_{1}^{\prime \prime}\right\|_{1}-\left\|\mathbb{U}_{2}^{\prime \prime}\right\|_{1}\right| \leq \varepsilon^{3}+2 \sqrt{M} \varepsilon^{2}+M \varepsilon .
$$

Next, we increase $\mu^{\prime \prime}$ to a measure $\mu$ on $\Omega_{1, \leq D}^{\prime} \times \Omega_{2, \leq D}^{\prime}$ by coupling $\mu_{1}^{\prime}-\mu_{1, D}^{\prime}$ and $\mu_{2}^{\prime}-\mu_{2, D}^{\prime}$ arbitrarily. Then $\mu$ has marginals $\left.\mu_{1}^{\prime}\right|_{\Omega_{1, \leq D}^{\prime}}$ and $\left.\mu_{2}^{\prime}\right|_{\Omega_{2, \leq D}^{\prime}}$ and $\mu-\widetilde{\delta} \leq$ $\mu^{\prime \prime} \leq \mu$. If we apply Lemma 3.16 , we then obtain a coupling of $\mathbb{W}_{1, \leq D}^{\prime}$ and $\mathbb{W}_{2, \leq D}^{\prime}$ such that the pullbacks $\mathbb{U}_{1}^{\prime \prime \prime}$ and $\mathbb{U}_{2}^{\prime \prime \prime}$ obey the bounds

$$
\left\|\mathbb{U}_{1}^{\prime \prime \prime}-\mathbb{U}_{2}^{\prime \prime \prime}\right\|_{2 \rightarrow 2, \mu} \leq \varepsilon+2 \sqrt{2 D \widetilde{\delta}},
$$

and

Setting

$$
|| \mathbb{U}_{1}^{\prime \prime \prime}\left\|_{1}-\right\| \mathbb{U}_{2}^{\prime \prime \prime} \|_{1} \mid \leq \varepsilon^{3}+2 \sqrt{M} \varepsilon^{2}+M \varepsilon+2 D \widetilde{\delta} .
$$

$$
\widetilde{C}=C+\varepsilon^{3}+2 \sqrt{M} \varepsilon^{2}+M \varepsilon+2 D \widetilde{\delta},
$$

we then have $\max \left\{\left\|\mathbb{U}_{1}^{\prime \prime \prime}\right\|_{1},\left\|\mathbb{U}_{2}^{\prime \prime \prime}\right\|_{1}\right\} \leq \widetilde{C}$ and hence

$$
\left\|D_{\mathbb{U}_{1}^{\prime \prime \prime}}-D_{\mathbb{U}_{2}^{\prime \prime \prime}}\right\|_{2, \mu}^{2} \leq\left(\varepsilon^{2}+\sqrt{M} \varepsilon\right)^{2}+4 \widetilde{C} \widetilde{\delta}+D^{2} \widetilde{\delta} .
$$

This completes the proof of (4).

Suppose $\left(\mathbb{W}_{n}\right)_{n=1}^{\infty}$ and $\mathbb{W}$ are graphexes over the $\sigma$-finite measure spaces $\boldsymbol{\Omega}_{n}=$ $\left(\Omega_{n}, \mathcal{F}_{n}, \mu_{n}\right)$ and $\boldsymbol{\Omega}=(\Omega, \mathcal{F}, \mu)$. Define, for any $D>0$,

$$
\Omega_{n, \leq D}=\left\{x \in \Omega_{n}: D_{\mathbb{W}_{n}}(x) \leq D\right\} \quad \text { and } \quad \Omega_{n,>D}=\left\{x \in \Omega_{n}: D_{\mathbb{W}_{n}}(x)>D\right\} .
$$

Recall that we have $\mu_{n}\left(\Omega_{n,>D}\right)<\infty$. Let $\mathbb{W}_{n, \leq D}$ consist of $\mathbb{W}_{n}$ restricted to $\Omega_{n, \leq D}$. Define $\Omega_{\leq D}, \Omega_{>D}$, and $\mathbb{W}_{\leq D}$ similarly. We then have the following.

Proposition 4.6. Given a sequence of graphexes $\mathbb{W}_{n}$ and a graphex $\mathbb{W}$ over the $\sigma$-finite measure spaces $\boldsymbol{\Omega}_{n}=\left(\Omega_{n}, \mathcal{F}_{n}, \mu_{n}\right)$ and $\boldsymbol{\Omega}=(\Omega, \mathcal{F}, \mu)$, respectively, define $\mathbb{W}_{n, \leq D}$ and $\mathbb{W}_{\leq D}$ as above. Then the following are equivalent.

(1) For all $D>0$ such that $\mu\left(\left\{D_{\mathbb{W}}=D\right\}\right)=0$, we have $\delta_{2 \rightarrow 2}\left(\mathbb{W}_{n, \leq D}, \mathbb{W}_{\leq D}\right) \rightarrow$ 0 and $\mu_{n}\left(\Omega_{n,>D}\right) \rightarrow \mu\left(\Omega_{>D}\right)$.

(2) The sequence is tight, and for all $D>0$ such that $\mu\left(\left\{D_{\mathbb{W}}=D\right\}\right)=0$, we have $\delta_{2 \rightarrow 2}\left(\mathbb{W}_{n, \leq D}, \mathbb{W}_{\leq D}\right) \rightarrow 0$.

(3) For every $\varepsilon>0$ and $n \in \mathbb{N}$ there exist subsets $\Omega_{n, \varepsilon} \subseteq \Omega_{n}$ and a subset $\Omega_{\varepsilon} \subseteq \Omega$ with $\mu_{n}\left(\Omega_{n} \backslash \Omega_{n, \varepsilon}\right) \leq \varepsilon$ and $\mu\left(\Omega \backslash \Omega_{\varepsilon}\right) \leq \varepsilon$, such that $\delta_{2 \rightarrow 2}\left(\mathbb{W}_{n}^{\prime}, \mathbb{W}^{\prime}\right) \rightarrow 0$, where $\mathbb{W}_{n}^{\prime}=\left.\left(\mathbb{W}_{n}\right)\right|_{\Omega_{n}, \varepsilon}$ and $\mathbb{W}^{\prime}=\left.\mathbb{W}\right|_{\Omega_{\varepsilon}}$.

(4) $\delta_{\diamond}\left(\mathbb{W}_{n}, \mathbb{W}\right) \rightarrow 0$.

Proof. (1) $\Rightarrow(2)$ : We have to prove tightness. For any $\varepsilon$, there exists a $D$ such that $\mu\left(\Omega_{>D}\right) \leq \varepsilon / 2$. Assuming without loss of generality that $D$ is chosen in such a way that $\mu\left(\left\{D_{\mathbb{W}}=D\right\}\right)=0$, we further have $\mu_{n}\left(\Omega_{n,>D}\right) \rightarrow \mu\left(\Omega_{>D}\right)$. This means that for all but a finite set of $n, \mu_{n}\left(\Omega_{n,>D}\right) \leq \varepsilon$. By increasing $D$, we can guarantee this for all $n$. Since $\delta_{2 \rightarrow 2}$ convergence implies in particular that $\left\|\mathbb{W}_{n, \leq D}\right\|_{1} \rightarrow\left\|\mathbb{W}_{\leq D}\right\|_{1}$, we have that $\left\|\mathbb{W}_{n, \leq D}\right\|_{1}$ is bounded. This proves property 3 from Theorem 4.1 . 
(2) clearly implies (3), because tightness implies that for any $\varepsilon$, there exists a $D$ such that the measure of points with degree greater than $D$ is at most $\varepsilon$, and we can increase $D$ to make sure that $\mu\left(\left\{D_{\mathbb{W}}=D\right\}\right)=0$.

To show that (3) implies (4), we note that the conditions in (3) imply that $\lim \sup _{n \rightarrow \infty} \delta_{\diamond}\left(\mathbb{W}_{n}, \mathbb{W}\right) \leq \sqrt{\varepsilon}$. Since $\varepsilon$ is arbitrary, this gives $(4)$.

It remains to show that (4) implies (1). The assumption $\delta_{\diamond}\left(\mathbb{W}_{n}, \mathbb{W}\right) \rightarrow 0$ implies that for all $\varepsilon>0$ there exists an $n_{0}$ such that for $n \geq n_{0}, \delta_{\diamond}\left(\mathbb{W}_{n}, \mathbb{W}\right)<\varepsilon$. Recalling Definition 2.14 and combining statements (1) and (3) of the previous lemma, this implies that for $D>\varepsilon+\varepsilon^{2}>0$ and $n \geq n_{0}$,

$$
\mu\left(D_{\mathbb{W}}>D+\varepsilon+\varepsilon^{2}\right)-2 \varepsilon^{2} \leq \mu_{n}\left(D_{\mathbb{W}_{n}}>D\right) \leq \mu\left(D_{\mathbb{W}}>D-\varepsilon^{2}-\varepsilon\right)+2 \varepsilon^{2} .
$$

By our assumption that $\mu\left(\left\{D_{\mathbb{W}}=D\right\}\right)=0$, we have that $D$ is a continuity point of the function $x \mapsto \mu\left(\left\{D_{\mathbb{W}}>x\right\}\right)$, showing that the upper and lower bound converge to $\mu\left(\left\{D_{\mathbb{W}}>D\right\}\right)$ as $\varepsilon \rightarrow 0$. This shows that $\mu_{n}\left(D_{\mathbb{W}_{n}}>D\right) \rightarrow \mu\left(\left\{D_{\mathbb{W}}>D\right\}\right)$ as $n \rightarrow \infty$.

Next we define

$$
M=\sup _{n} \mu_{n}\left(\Omega_{n,>D}\right)+\mu\left(\Omega_{>D}\right) \quad \text { and } \quad \delta(\varepsilon)=\mu\left(\left\{D-\varepsilon-\varepsilon^{2}<D_{\mathbb{W}} \leq D+\varepsilon+\varepsilon^{2}\right\}\right) .
$$

Note that $M$ is finite by the fact that $\mu_{n}\left(\Omega_{n,>D}\right) \rightarrow \mu\left(\Omega_{>D}\right)$, and that $\delta(\varepsilon) \rightarrow 0$ as $\varepsilon \rightarrow 0$ by the fact that $\mu\left(\left\{D_{\mathbb{W}}=D\right\}\right)=0$. We now apply the previous lemma (with $W_{1}$ replaced by $\mathbb{W}_{n}$ and $\mathbb{W}_{2}$ replaced by $\left.\mathbb{W}\right)$ to conclude the proof.

The following proposition is an easy corollary of Proposition 4.6.

Proposition 4.7. Given two graphexes $\mathbb{W}_{1}, \mathbb{W}_{2}$, let $\mathbb{W}_{i, \leq D}$ be the graphex $\mathbb{W}_{i}$ restricted to $\Omega_{i, \leq D}=\left\{x \in \Omega_{i}: D_{\mathbb{W}_{i}}(x) \leq D\right\}$. Then the following are equivalent.

(1) For any $D>0$,

$$
\mu_{1}\left(\Omega_{1} \backslash \Omega_{1, \leq D}\right)=\mu_{2}\left(\Omega_{2} \backslash \Omega_{2, \leq D}\right) \text { and } \delta_{2 \rightarrow 2}\left(\mathbb{W}_{1, \leq D}, \mathbb{W}_{2, \leq D}\right)=0 .
$$

(2) For any $D>0, \delta_{2 \rightarrow 2}\left(\mathbb{W}_{1, \leq D}, \mathbb{W}_{2, \leq D}\right)=0$

(3) For any $\varepsilon>0$, there exist subsets $\Omega_{1, \varepsilon} \subseteq \Omega_{1}, \Omega_{2, \varepsilon} \subseteq \Omega_{2}$ with $\mu_{i}\left(\Omega_{i} \backslash \Omega_{i, \varepsilon}\right) \leq \varepsilon$, such that if $\mathbb{W}_{i}^{\prime}$ is the restriction to $\Omega_{i} \backslash \Omega_{i, \varepsilon}$, then $\delta_{2 \rightarrow 2}\left(\mathbb{W}_{1}^{\prime}, \mathbb{W}_{2}^{\prime}\right) \leq \varepsilon$.

(4) $\delta_{\diamond}\left(\mathbb{W}_{1}, \mathbb{W}_{2}\right)=0$.

It is easy to see that this is an equivalence relation.

Proof. Observing that the functions $f_{i}: \mathbb{R}_{+} \rightarrow \mathbb{R}+$ defined by $D \mapsto \mu_{i}\left(\Omega_{i} \backslash \Omega_{i, \leq D}\right)$ for $i=1,2$ are equal if and only if $f_{1}(D)=f_{2}(D)$ for all continuity points of $f_{1}$, this follows by applying Proposition 4.6 with $\mathbb{W}=\mathbb{W}_{1}$ and each $\mathbb{W}_{n}=\mathbb{W}_{2}$.

Remark 4.8. It is not a priori clear how to generalize the notion of tightness to signed graphexes, even if we restrict ourselves to the case where the graphon parts are uniformly bounded, for example by taking graphons that take values in $[-1,1]$. Indeed, recalling Lemma 3.12 and the role it played in showing that $\delta_{\diamond}$ is finite for signed graphexes with bounded graphon part, one might want to modify Definition 2.20 for such graphexes by replacing the notion of $D$-bounded marginals by that of $D$-bounded absolute marginals, since this would, in particular, guarantee that a finite set of signed graphexes with graphon parts in $[-1,1]$ is tight. It would also make the generalization of several of our results straightforward, since this definition just reduces the notion of tightness of a set of graphexes $\mathcal{S}$ to the set of graphexes $\mathcal{S}^{\prime}=\{|\mathbb{W}|: \mathbb{W} \in \mathcal{S}\}$. 
The following example shows that this straightforward generalization of Definition 2.20 to signed graphexes does not give a characterization of precompact sets with respect to the metric $\delta_{\diamond}$, as it did for unsigned graphexes; see Theorem 2.21.

Let $W_{n}$ be equal to $n^{-3 / 4}$ on $[0,1) \times[1, n+1) \cup[1, n+1) \times[0,1)$, equal to $-n^{-3 / 4}$ on $[0,1) \times[n+1,2 n+2) \cup[n+1,2 n+1) \times[0,1)$, and zero everywhere else on $\mathbb{R}_{+}^{2}$. Define $\mathbb{W}_{n}$ to be the graphex with graphon part $W_{n}$ and zero star and dust part. Then $D_{\mathbb{W}_{n}}$ is equal to $n^{-3 / 4}$ on $[1, n+1)$, equal to $-n^{-3 / 4}$ on $[n+1,2 n+1)$, and 0 everywhere else. Finally, $\rho\left(\mathbb{W}_{n}\right)=0$ for all $n$. Since $\left\|W_{n}\right\|_{2}=2 n^{-1 / 2}$ and $\left\|D_{\mathbb{W}_{n}}\right\|_{2}=\sqrt{2} n^{-1 / 2}, \mathbb{W}_{n}$ tends to the zero graphex in the metric $\delta_{2 \rightarrow 2}$ and hence also in $\delta_{\diamond}$. But $\left\|\mathbb{W}_{n}\right\|_{1}=\|W\|_{1}=4 n^{1 / 4} \rightarrow \infty$, a fact which can't be changed by removing just a part of measure $\varepsilon$ from the underlying space, $\mathbb{R}_{+}$. This shows that with the obvious generalization of Definition 2.20 to signed graphexes not all sequences of signed graphexes that are convergent in $\delta_{2 \rightarrow 2}$ or $\delta_{\diamond}$ are tight.

We therefore believe that a complete theory of signed graphexes, even in the simplified case where all graphons take values in $[-1,1]$, requires either a modification of the metric, or modification of the notion of tightness. We leave this problem as an open research problem.

\section{Regularity LEMMA AND COMPACTNESS}

In this section, we will prove a regularity lemma (Theorem 5.3 below), and use it to prove Theorem 2.21, which in turn is an important ingredient in our proof that GP-convergence and $\delta_{\diamond}$-convergence are equivalent. To state the regularity lemma, we recall that a finite subspace partition of a measure space $\boldsymbol{\Omega}=(\Omega, \mathcal{F}, \mu)$ is a partition of a measurable subset of $\Omega$ into finitely many measurable subsets of finite measure. Throughout this section, we will use the notation $\mathscr{P}=\left(\Omega_{\mathscr{P}}, \mathcal{P}\right)$ for a finite subspace partition, with $\Omega_{\mathscr{P}}$ denoting the subset of $\Omega$, and $\mathcal{P}=\left(P_{1}, \ldots, P_{m}\right)$ denoting the partition of $\Omega_{\mathscr{P}}$. We will also need the notion of refinement.

Definition 5.1. Given two subspace partitions $\mathscr{P}=\left(\Omega_{\mathscr{P}}, \mathcal{P}\right)$ and $\mathscr{Q}=\left(\Omega_{\mathcal{Q}}, \mathcal{Q}\right)$, we say that $\mathscr{P}$ refines $\mathscr{Q}$ if $\Omega_{\mathcal{Q}} \subseteq \Omega_{\mathscr{P}}$ and $\mathcal{P}$ is a refinement of $\mathcal{Q} \cup\left\{\Omega_{\mathscr{P}} \backslash \Omega_{\mathcal{Q}}\right\}$.

Given an integrable, signed graphex $\mathbb{W}$, a subspace partition $\mathscr{P}$ naturally generates a step function $\mathbb{W}_{\mathscr{P}}$ by "averaging". The precise definition is as follows.

Definition 5.2. Given a signed graphex $\mathbb{W}=(W, S, I, \boldsymbol{\Omega})$ and a finite subspace partition $\mathscr{P}=\left(\Omega_{\mathscr{P}}, \mathcal{P}\right)$, take $\mathbb{W}_{\mathscr{P}}$ to be the signed graphex $\mathbb{W}_{\mathscr{P}}=\left(W_{\mathscr{P}}, S_{\mathscr{P}}, I_{\mathscr{P}}, \boldsymbol{\Omega}\right)$ defined by

$$
\begin{gathered}
I_{\mathscr{P}}=\frac{1}{2} \int_{\left(\Omega \backslash \Omega_{\mathscr{P}}\right) \times\left(\Omega \backslash \Omega_{\mathscr{P}}\right)} W(x, y) d \mu(x) d \mu(y)+\int_{\Omega \backslash \Omega_{\mathscr{P}}} S(x) d \mu(x)+I, \\
S_{\mathscr{P}}(x)=\frac{1}{\mu\left(P_{i}\right)} \int_{P_{i}}\left(S(x)+\int_{\Omega \backslash \Omega_{\mathscr{P}}} W(x, y) d \mu(y)\right) d \mu(x) \quad \text { if } \quad x \in P_{i}
\end{gathered}
$$

for some $i \in\{1, \ldots, k\}$, and 0 everywhere else, and

$$
W_{\mathscr{P}}(x, y)=\frac{1}{\mu\left(P_{i}\right) \mu\left(P_{j}\right)} \int_{P_{i} \times P_{j}} W\left(x^{\prime}, y^{\prime}\right) d \mu\left(x^{\prime}\right) d \mu\left(y^{\prime}\right) \quad \text { if } \quad(x, y) \in P_{i} \times P_{j}
$$

for some $i, j \in\{1, \ldots, k\}$ and $W_{\mathscr{P}}(x, y)=0$ everywhere else. 
Note that with this definition, for $x \in P_{i}$,

$$
D_{\mathbb{W}_{\mathscr{P}}}(x)=\frac{1}{\mu\left(P_{i}\right)} \int_{P_{i}} D_{\mathbb{W}}(x) d \mu(x) .
$$

We also have $\rho\left(\mathbb{W}_{\mathscr{P}}\right)=\rho(\mathbb{W})$, as well as $\left\|\mathbb{W}_{\mathcal{P}}\right\|_{1} \leq\|\mathbb{W}\|_{1},\left\|D_{\mathbb{W}_{\mathscr{P}}}\right\|_{\infty} \leq\left\|D_{\mathbb{W}}\right\|_{\infty}$, and $\left\|W_{\mathscr{P}}\right\|_{\infty} \leq\|W\|_{\infty}$.

Theorem 5.3. For any $B, C, D<\infty$, and $\varepsilon>0$, there exists an $M(\varepsilon)$ and $N(\varepsilon)$ such that for any signed graphex $\mathbb{W}$ that is $(B, C, D)$-bounded there exists a partition $\left(\Omega_{\mathscr{P}}, \mathcal{P}\right)$ with $\mathcal{P}=\left\{P_{1}, \ldots, P_{m}\right\}, m \leq M(\varepsilon)$, and $\mu\left(\Omega_{\mathscr{P}}\right) \leq N(\varepsilon)$ such that

$$
d_{\bigotimes}(\mathbb{W}, \mathbb{W} \mathscr{P}) \leq \varepsilon .
$$

We can take

$$
M(\varepsilon)=2^{(2 B C+C D) / \varepsilon^{2}} \quad \text { and } \quad N(\varepsilon)=\left(4 C^{3} D+8 B C^{2} D\right) / \varepsilon^{4} .
$$

Given any finite subspace partition $\mathscr{Q}=\left(\Omega_{\mathscr{Q}}, \mathcal{Q}\right)$, we can require the subspace partition $\mathscr{P}=\left(\Omega_{\mathscr{P}}, \mathcal{P}\right)$ to be a refinement of $\mathscr{Q}$. In this case, the bound on the number of parts is $|\mathcal{Q}| M(\varepsilon)$ and the bound on $\mu\left(\Omega_{\mathscr{P}}\right)$ is $\mu\left(\Omega_{\mathcal{Q}}\right)+N(\varepsilon)$.

Proof. Motivated by the original proof of the weak regularity lemma (see in particular the proof of Theorem 12 in [14], which to our knowledge is the first place where a weak regularity lemma for functions $W:[0,1]^{2} \rightarrow \mathbb{R}$ was established), we construct a sequence of partitions $\mathscr{P}_{0}, \mathscr{P}_{1}, \ldots, \mathscr{P}_{\ell}$ such that eventually, we must have that $d_{\bigotimes}\left(\mathbb{W}, \mathbb{W}_{\mathscr{P}_{\ell}}\right) \leq \varepsilon$. We start with the trivial partition $\mathscr{P}_{0}=(\emptyset, \emptyset)$ (so that $\mathbb{W}_{\mathscr{P}_{0}}$ is the graphex with zero graphon and star part, and dust part $\rho(\mathbb{W}))$, and then construct a sequence of refinements $\mathscr{P}_{0}, \mathscr{P}_{1}, \ldots, \mathscr{P}_{\ell}$.

In a preliminary step, we claim that for any partition $\mathscr{P}$,

$$
\left\langle W-W_{\mathscr{P}}, W_{\mathscr{P}}\right\rangle=\int_{\Omega \times \Omega}\left(W(x, y)-W_{\mathscr{P}}(x, y)\right) W_{\mathscr{P}}(x, y) d x d y=0
$$

and

$$
\left\langle D_{\mathbb{W}}-D_{\mathbb{W}_{\mathscr{P}}}, D_{\mathbb{W}_{\mathscr{P}}}\right\rangle=\int_{\Omega}\left(D_{\mathbb{W}}(x)-D_{\mathbb{W}_{\mathscr{P}}}(x)\right) D_{\mathbb{W}_{\mathscr{P}}}(x) d x=0
$$

This follows from the fact that for any pair of finite parts $P_{i}, P_{j}, W_{\mathscr{P}}$ and $D_{\mathbb{W}_{\mathscr{P}}}$ are constant, and the integral of $W-W_{\mathscr{P}}$ and $D_{\mathbb{W}}-D_{\mathbb{W}_{\mathscr{P}}}$ is zero. Since $W_{\mathscr{P}}$ is zero between pairs of parts where at least one is non-finite, and $D_{\mathbb{W}_{\mathscr{P}}}$ is zero on non-finite parts, this implies the claim. Therefore, we have

$$
\|W\|_{2}^{2}=\left\|W_{\mathscr{P}}\right\|_{2}^{2}+\left\|W-W_{\mathscr{P}}\right\|_{2}^{2}
$$

and

$$
\left\|D_{\mathbb{W}}\right\|_{2}^{2}=\left\|D_{\mathbb{W}_{\mathscr{P}}}\right\|_{2}^{2}+\left\|D_{\mathbb{W}}-D_{\mathbb{W}_{\mathscr{P}}}\right\|_{2}^{2} .
$$

If we have a finite subspace partition $\mathscr{P}=\left(\Omega_{\mathscr{P}}, \mathcal{P}\right)$ and a refinement $\mathscr{P}^{\prime}$, it is then easy to check that $\left(\mathbb{W}_{\mathscr{P} \prime}\right)_{\mathscr{P}}=\mathbb{W}_{\mathscr{P}}$; therefore, the same properties hold for $\mathbb{W}_{\mathscr{P}}$ and $\mathbb{W}_{\mathscr{P}^{\prime}}$.

Suppose now that we have constructed a sequence of refinements $\mathscr{P}_{0}, \mathscr{P}_{1}, \ldots, \mathscr{P}_{i}$ such that $d_{凶}\left(\mathbb{W}, \mathbb{W}_{\mathscr{P}_{j}}\right)>\varepsilon$ for all $j \leq i$. Then we in particular have that $d_{\rrbracket}\left(\mathbb{W}, \mathbb{W}_{\mathscr{P}_{i}}\right)>\varepsilon$, which implies that $\left\|D_{\mathbb{W}}-D_{\mathbb{W}_{\mathscr{P}_{i}}}\right\|_{\otimes}>\varepsilon$ or $\left\|W-W_{\mathscr{P}_{i}}\right\|_{\otimes}>\varepsilon$. If the former holds, then there exists a set $S \subseteq \Omega$ (of finite measure) such that

$$
\frac{1}{\sqrt{\mu(S)}}\left|\int_{S} D_{\mathbb{W}}(x) d \mu(x)-\int_{S} D_{\mathbb{W}_{\mathscr{P}}}(x) d \mu(x)\right|=A>\varepsilon .
$$


Let $\mathscr{P}_{i+1}=\left(\Omega_{\mathscr{P}_{i+1}}, \mathcal{P}_{i+1}\right)$ with $\Omega_{\mathscr{P}_{i+1}}=\Omega_{\mathscr{P}_{i}} \cup S$ and let $\mathcal{P}_{i+1}$ be the partition that refines each part of $\mathcal{P}_{i}$ by the intersection with $S$; in particular, this divides each part into at most 2 parts, and $\mu\left(\Omega_{\mathscr{P}_{i+1}}\right) \leq \mu\left(\Omega_{\mathscr{P}_{i}}\right)+\mu(S)$. We also have

$$
\frac{1}{\sqrt{\mu(S)}}\left|\int_{S} D_{\mathbb{W}_{\mathscr{P}_{i+1}}}(x) d \mu(x)-\int_{S} D_{\mathbb{W}_{\mathscr{P}_{i}}}(x) d \mu(x)\right|=A \text {. }
$$

Therefore,

$$
\left\|D_{\mathbb{W}_{\mathscr{P}_{i+1}}}-D_{\mathbb{W}_{\mathscr{P}_{i}}}\right\|_{2}^{2} \geq\left\langle D_{\mathbb{W}_{\mathscr{P}_{i+1}}}-D_{\mathbb{W}_{\mathscr{P}_{i}}}, \frac{\chi_{S}}{\sqrt{\mu(S)}}\right\rangle^{2}=A^{2}
$$

Overall, this implies that we have

$$
\left\|D_{\mathbb{W}_{\mathscr{P}_{i}}}\right\|_{2}^{2}+\varepsilon^{2} \leq\left\|D_{\mathbb{W}_{\mathscr{P}_{i+1}}}\right\|_{2}^{2} \leq\left\|D_{\mathbb{W}}\right\|_{2}^{2}
$$

We also have

$$
\left\|W_{\mathscr{P}_{i}}\right\|_{2}^{2} \leq\left\|W_{\mathscr{P}_{i+1}}\right\|_{2}^{2} \leq\|W\|_{2}^{2}
$$

If $\left\|W-W_{\mathscr{P}_{i}}\right\|_{\otimes}>\varepsilon$, then there exist sets $S, T \subseteq \Omega$ (of finite measure) such that

$$
\frac{1}{\sqrt{\mu(S) \mu(T)}}\left|\int_{S \times T} W(x, y) d \mu(x) d \mu(y)-\int_{S \times T} W_{\mathscr{P}_{i}}(x, y) d \mu(x) d \mu(y)\right|>\varepsilon .
$$

Let $\mathscr{P}_{i+1}=\left(\Omega_{\mathscr{P}_{i+1}}, \mathcal{P}_{i+1}\right)$ with $\Omega_{\mathscr{P}_{i+1}}=\Omega_{\mathscr{P}} \cup S \cup T$ and let $\mathcal{P}_{i+1}$ be the partition that refines each part of $\mathcal{P}_{i}$ by the intersection with $S$ and $T$, in particular, this refines each part into at most 4 parts, and $\mu\left(\Omega_{\mathscr{P}_{i+1}}\right) \leq \mu\left(\Omega_{\mathscr{P}_{i}}\right)+\mu(S)+\mu(T)$. Proceeding as before, we have

$$
\left\|W_{\mathscr{P}_{i}}\right\|_{2}^{2}+\varepsilon^{2} \leq\left\|W_{\mathscr{P}_{i+1}}\right\|_{2}^{2} \leq\|W\|_{2}^{2}
$$

and furthermore,

$$
\left\|D_{\mathbb{W}_{\mathscr{P}_{i}}}\right\|_{2} \leq\left\|D_{\mathbb{W}_{\mathscr{P}_{i+1}}}\right\|_{2} \leq\left\|D_{\mathbb{W}}\right\|_{2} .
$$

The first step can occur at most $\left\|D_{\mathbb{W}}\right\|_{2} / \varepsilon^{2}$ times, and the second at most $\|W\|_{2}^{2} / \varepsilon^{2}$ times. Since in the first step, the number of partition classes at most doubles, and in the second it goes up by at most a factor of four, this proves that there exists a partition $\mathscr{P}$ with at most

$$
2^{\left(2\|W\|_{2}^{2}+\left\|D_{\mathbb{W}}\right\|_{2}^{2}\right) / \varepsilon^{2}} \leq 2^{(2 B C+C D) / \varepsilon^{2}}=M(\varepsilon)
$$

classes such that $d_{\rrbracket}(\mathbb{W}, \mathbb{W} \mathscr{P}) \leq \varepsilon$.

To prove that $\mu\left(\Omega_{\mathscr{P}}\right) \leq N(\varepsilon)$, we claim that in each step, (5.1) implies that

$$
\mu(S) \leq \frac{4 C^{2}}{\varepsilon^{2}}
$$

and (5.2) implies that

Indeed, for any $S \subseteq \Omega$,

$$
\mu(S), \mu(T) \leq \frac{4 C D}{\varepsilon^{2}} .
$$

$$
\left|\int_{S} D_{\mathbb{W}}(x)-D_{\mathbb{W}_{\mathcal{P}_{i}}}(x)\right| \leq 2 C .
$$

This implies that if

$$
\frac{1}{\sqrt{\mu(S)}}\left|\int_{S} D_{\mathbb{W}}(x) d \mu(x)-\int_{S} D_{\mathbb{W}_{\mathscr{P}_{i}}}(x) d \mu(x)\right| \geq \varepsilon
$$


then

$$
\mu(S) \leq \frac{4 C^{2}}{\varepsilon^{2}}
$$

On the other hand,

$$
\left|\int_{S \times T} W-W_{\mathscr{P}}\right| \leq 2 C \quad \text { and } \quad\left|\int_{S \times T} W-W_{\mathscr{P}}\right| \leq 2 D \mu(T) .
$$

Therefore,

This implies that if

$$
\left|\int_{S \times T} W-W_{\mathscr{P}}\right| \leq 2 \sqrt{C D \mu(T)}
$$

then

$$
\frac{1}{\sqrt{\mu(S) \mu(T)}}\left|\int_{S \times T} W-W_{\mathscr{P}_{i}}\right| \geq \varepsilon
$$

$$
\mu(S) \leq \frac{4 C D}{\varepsilon^{2}}
$$

The bound for $\mu(T)$ follows similarly.

Since first step can occur at most $\left\|D_{\mathbb{W}}\right\|_{2} / \varepsilon^{2}$ times, and the second at most $\|W\|_{2}^{2} / \varepsilon^{2}$ times, this shows that

$$
\mu\left(\Omega_{\mathscr{P}}\right) \leq \frac{4 C^{2}}{\varepsilon^{2}} \frac{\left\|D_{\mathbb{W}}\right\|_{2}^{2}}{\varepsilon^{2}}+2 \frac{4 C D}{\varepsilon^{2}} \frac{\|W\|_{2}^{2}}{\varepsilon^{2}} \leq \frac{4}{\varepsilon^{4}}\left(C^{3} D+2 B C^{2} D\right)=N(\varepsilon) .
$$

The second statement follows by choosing $\mathscr{P}_{0}=\mathscr{Q}$.

Remark 5.4. With the help of Proposition 3.19, Theorem 5.3 can immediately be transformed into a similar statement for the kernel distance $d_{2 \rightarrow 2}(\mathbb{W}, \mathbb{W} \mathscr{P})$, provided $N(\varepsilon)$ and $M(\varepsilon)$ are replaced by bounds of the form $M(\varepsilon)=2^{c / \varepsilon^{8}}$ and $N(\varepsilon)=d / \varepsilon^{16}$ where $c$ and $d$ are constants depending on $B, C$ and $D$.

We would like to prove a version of this "regularity lemma" for $d_{2 \rightarrow 2}$ where the parts have equal size. We first show some preliminary lemmas.

Lemma 5.5. Let $\mathbb{W}_{1}, \mathbb{W}_{2}$ be two graphexes on the same space $\boldsymbol{\Omega}$, and let $\mathscr{P}$ be a finite subspace partition of $\boldsymbol{\Omega}$. Then

$$
\left\|W_{1, \mathscr{P}}-W_{2, \mathscr{P}}\right\|_{2 \rightarrow 2} \leq\left\|W_{1}-W_{2}\right\|_{2 \rightarrow 2}
$$

and

$$
\left\|D_{\mathbb{W}_{1, \mathscr{P}}}-D_{\mathbb{W}_{2, \mathscr{P}}}\right\|_{2} \leq\left\|D_{\mathbb{W}_{1}}-D_{\mathbb{W}_{2}}\right\|_{2}
$$

Proof. Note that

$$
\begin{aligned}
\left\|W_{1, \mathscr{P}}-W_{2, \mathscr{P}}\right\|_{2 \rightarrow 2} & =\sup _{\substack{f, g \in L^{2}(\Omega) \\
\|f\|_{2}=\|g\|_{2}=1}} f \circ\left(W_{1, \mathscr{P}}-W_{2, \mathscr{P}}\right) \circ g \\
& =\sup _{\substack{f, g \in L^{2}(\Omega \mathscr{P}) \\
\|f\|_{2}=\|g\|_{2}=1}} f \circ\left(W_{1, \mathscr{P}}-W_{2, \mathscr{P}}\right) \circ g .
\end{aligned}
$$

If we let $f_{\mathscr{P}}$ and $g_{\mathscr{P}}$ consist of the average values of $f$ and $g$ on each part of $\mathcal{P}$ and zero outside $\Omega_{\mathscr{P}}$, then

$$
f \circ\left(W_{1, \mathscr{P}}-W_{2, \mathscr{P}}\right) \circ g=f_{\mathscr{P}} \circ\left(W_{1, \mathscr{P}}-W_{2, \mathscr{P}}\right) \circ g_{\mathscr{P}}=f_{\mathscr{P}} \circ\left(W_{1}-W_{2}\right) \circ g_{\mathscr{P}},
$$

which implies the first claim. The second claim follows similarly. 
Corollary 5.6. Suppose that $\mathbb{W}$ is a graphex, and that $\mathbb{U}$ is a step graphex over the same space as $\mathbb{W}$. If $\mathscr{P}=\left(\Omega_{\mathscr{P}}, \mathcal{P}\right)$ is a finite subspace partition such that $\mathbb{U}$ is constant on each part of $\mathcal{P}$ and zero outside $\Omega_{\mathscr{P}}$, then

$$
\left\|W_{\mathscr{P}}-W\right\|_{2 \rightarrow 2} \leq 2\|U-W\|_{2 \rightarrow 2}
$$

and

$$
\left\|D_{\mathbb{W} \mathscr{P}}-D_{\mathbb{W}}\right\|_{2} \leq 2\left\|D_{\mathbb{U}}-D_{\mathbb{W}}\right\|_{2} .
$$

Proof. Note that $\mathbb{U}_{\mathscr{P}}=\mathbb{U}$. We have

$$
\begin{aligned}
\left\|W_{\mathscr{P}}-W\right\|_{2 \rightarrow 2} & \leq\left\|W_{\mathscr{P}}-U\right\|_{2 \rightarrow 2}+\|U-W\|_{2 \rightarrow 2} \\
& =\left\|W_{\mathscr{P}}-U \mathscr{P}\right\|_{2 \rightarrow 2}+\|U-W\|_{2 \rightarrow 2} \leq 2\|U-W\|_{2 \rightarrow 2} .
\end{aligned}
$$

Similarly,

$$
\begin{aligned}
\left\|D_{\mathbb{W}_{\mathscr{P}}}-D_{\mathbb{W}}\right\|_{2} & \leq\left\|D_{\mathbb{W}_{\mathscr{P}}}-D_{\mathbb{U}}\right\|_{2}+\left\|D_{\mathbb{U}}-D_{\mathbb{W}}\right\|_{2} \\
& =\left\|D_{\mathbb{W}_{\mathscr{P}}}-D_{\mathbb{U}_{\mathscr{P}}}\right\|_{2}+\left\|D_{\mathbb{U}}-D_{\mathbb{W}}\right\|_{2} \leq 2\left\|D_{\mathbb{U}}-D_{\mathbb{W}}\right\|_{2} .
\end{aligned}
$$

Theorem 5.7. Given $B, C, D$, and $\varepsilon>0$, there exists $\rho_{0}=\rho_{0}(\varepsilon, B, C, D)>0$ and $N_{0}=N_{0}(\varepsilon, B, C, D)$ such that for any $\rho<\rho_{0}$, any $m \geq N_{0} / \rho$, and any $(B, C, D)$-bounded signed graphex $\mathbb{W}$ on an atomless space with infinite measure, there exists a subspace partition $\mathscr{P}=\left(\Omega_{\mathscr{P}}, \mathcal{P}\right)$ with exactly $m$ parts of size $\rho$ such that $d_{2 \rightarrow 2}\left(\mathbb{W}, \mathbb{W}_{\mathscr{P}}\right) \leq \varepsilon$. If $\mathscr{P}_{0}=\left(\Omega_{\mathscr{P}_{0}}, \mathcal{P}_{0}\right)$ is an arbitrary finite subspace partition, we can require $\mathscr{P}$ to refine $\mathscr{P}_{0}$, as long as each part of $\mathcal{P}_{0}$ is divisible by $\rho$ (and increasing the bound on $N_{0}(\varepsilon)$ and decreasing $\rho_{0}$ appropriately depending on $\left|\mathcal{P}_{0}\right|$ and $\mu\left(\Omega_{\mathscr{P}_{0}}\right)$ ).

Proof. Apply Theorem 5.3 and Proposition 3.19 to obtain a subspace partition $\mathscr{P}^{\prime}$ with at most $M(\varepsilon / 3)$ parts and size at most $N(\varepsilon / 3)$ such that $d_{2 \rightarrow 2}\left(\mathbb{W}, \mathbb{W}_{\mathscr{P}^{\prime}}\right) \leq \varepsilon / 3$. We first construct a refinement $\mathscr{Q}=\left(\Omega_{\mathscr{Q}}, \mathcal{Q}\right)$ as follows. Add a part from $\Omega \backslash \Omega_{\mathscr{P}}$, so that the total measure of $\Omega_{\mathscr{Q}}$ is equal to $m \rho$ (this will require $m \geq N(\varepsilon / 3) / \rho$ ), and divide each part of $\mathcal{P}^{\prime} \cup\left\{\Omega_{\mathscr{Q}} \backslash \Omega_{\mathscr{P}^{\prime}}\right\}$ into parts of size $\rho$, with perhaps one part remaining of smaller size. We then define $\mathscr{P}$ by combining the parts of $\mathcal{Q}$ that have size smaller than $\rho$, including the part added from $\Omega \backslash \Omega_{\mathscr{P}}$, into a single set $\Omega^{\prime}$, and then dividing $\Omega^{\prime}$ into parts of size $\rho$ (and keeping the remaining parts of $\mathscr{Q}$ ). Then $\mathbb{W}_{\mathcal{Q}}$ and $\mathbb{W}_{\mathcal{P}}$ differ only on $\Omega^{\prime}$, which has size at most $(M(\varepsilon / 3)+1) \rho$, implying that

$$
\begin{aligned}
\left\|W_{\mathscr{Q}}-W_{\mathscr{P}}\right\|_{2 \rightarrow 2}^{2} & \leq\left\|W_{\mathscr{Q}}-W_{\mathscr{P}}\right\|_{2}^{2} \\
& \leq 2 \int_{\Omega \times \Omega^{\prime}}\left(W_{\mathscr{Q}}-W_{\mathscr{P}}\right)^{2} \leq 2 \int_{\Omega \times \Omega^{\prime}} B\left(\left|W_{\mathscr{Q}}\right|+\left|W_{\mathscr{P}}\right|\right) \\
& \leq 4 B \int_{\Omega^{\prime}} D_{|W|} \leq 4(M(\varepsilon / 3)+1) \rho B D .
\end{aligned}
$$

We also have

$$
\left\|D_{\mathbb{W}_{\mathscr{Q}}}-D_{\mathbb{W}_{\mathscr{P}}}\right\|_{2} \leq 2 \sqrt{\int_{\Omega^{\prime}} D_{\left|\mathbb{W}_{\mid}\right|}(x)^{2} d \mu(x)} \leq 2 \sqrt{(M(\varepsilon / 3)+1) \rho} D .
$$

Furthermore, we know that $\mathbb{W}_{\mathscr{P}}$ is constant on each part of $\mathscr{Q}$. Therefore, applying Corollary 5.6, we have

$$
\left\|W-W_{\mathscr{Q}}\right\|_{2 \rightarrow 2} \leq 2\left\|W-\mathbb{W}_{\mathscr{P}}\right\|_{2 \rightarrow 2} \leq 2 \varepsilon / 3
$$

and

$$
\left\|D_{\mathbb{W}}-D_{\mathbb{W}_{\mathcal{Q}}}\right\|_{2} \leq 2\left\|D_{\mathbb{W}}-D_{\mathbb{W}_{\mathscr{P}^{\prime}}}\right\|_{2} \leq 2 \varepsilon^{2} / 9
$$


Therefore, if $\rho$ is small enough, then

$$
\left\|W-W_{\mathscr{P}}\right\|_{2 \rightarrow 2} \leq \varepsilon
$$

and

$$
\left\|D_{\mathbb{W}}-D_{\mathbb{W}_{\mathscr{P}}}\right\|_{2} \leq \varepsilon^{2}
$$

We can add parts of zero measure and the above argument still works. If we want $\mathscr{P}$ to be a refinement of a starting partition $\mathscr{P}_{0}$, we apply Theorem 5.3 and Proposition 3.19 and make sure to combine the leftover parts so that they are within the same part of $\mathcal{P}_{0}$, this can be done since each part of $\mathcal{P}_{0}$ is divisible by $\rho$.

We close this section by proving Theorem 2.21. To this end, we will first establish two lemmas.

Lemma 5.8. Let $\mathcal{S}$ be a set of signed graphexes over atomless spaces of infinite measure such that the following condition holds:

(a) For every $\varepsilon>0$, there exists $B, C, D$ such that for any $\mathbb{W}=(W, S, I,(\Omega, \mathcal{F}, \mu)) \in$ $\mathcal{S}$, taking $\Omega_{\leq D}$ to be the set of points with $D_{|\mathbb{W}|}(x) \leq D$, we have that $\mu(\Omega \backslash$ $\left.\Omega_{\leq D}\right) \leq \varepsilon,\left\|\left.W\right|_{\Omega_{\leq D}}\right\|_{\infty} \leq B$, and $\left\|\left.\mathbb{W}\right|_{\Omega_{\leq D}}\right\|_{1} \leq C$.

Then there exist strictly increasing sequences of integers $a_{k} \geq 2 k$ and $b_{k} \geq k$ and sequences of positive real numbers $B_{k}, C_{k}$, and $D_{k}$ such that the following holds. For any graphex $\mathbb{W} \in \mathcal{S}, \mathbb{W}=(W, S, I, \boldsymbol{\Omega})$ with $\boldsymbol{\Omega}=(\Omega, \mathcal{F}, \mu)$, there exists a sequence of subsets $P_{k, 0} \subseteq \Omega$ and subspace partitions $\mathscr{P}_{k}=\left(\Omega_{\mathscr{P}_{k}}, \mathcal{P}_{k}\right), \mathcal{P}_{k}=\left\{P_{k, 1}, \ldots, P_{k, m_{k}}\right\}$ with $m_{k}=2^{a_{k}+b_{k}}$, such that for all $k, \Omega_{\mathscr{P}_{k}}$ is disjoint from $P_{k, 0}$ and

(1) $P_{k+1,0} \subseteq P_{k, 0}$ and $\mu\left(P_{k, 0}\right)=2^{-2 k}$,

(2) $\mathbb{W}_{k}=\left.\mathbb{W}\right|_{\Omega \backslash P_{k, 0}}$ is $\left(B_{k}, C_{k}, D_{k}\right)$-bounded,

(3) $\mathscr{P}_{k+1}$ refines $\mathscr{P}_{k}$,

(4) $P_{k, i}$ for $i \geq 1$ has measure $2^{-a_{k}}$, and

(5) $d_{2 \rightarrow 2}\left(\mathbb{W}_{k},\left(\mathbb{W}_{k}\right)_{\mathscr{P}_{k}}\right) \leq 2^{-k}$.

Note that by property (3) from Theorem 4.1, every tight set of unsigned graphexes obeys the condition (a) (with $B=1$ ), showing that the conclusions of the theorem hold for any tight set $\mathcal{S}$ of graphexes over atomless spaces of infinite measure.

Proof. Define

$$
D_{k}=\inf \left\{D: \text { for all } \mathbb{W} \in \mathcal{S}, \mu\left(\Omega \backslash \Omega_{\leq D}\right) \leq 2^{-2 k}\right\},
$$

and let $B_{k}=\sup _{\mathbb{W} \in \mathcal{S}}\left\|\left.W\right|_{\Omega_{\leq D_{k}}}\right\|_{\infty}$ and $C_{k}=\sup _{\mathbb{W} \in \mathcal{S}}\left\|\left.\mathbb{W}\right|_{\Omega_{\leq D_{k}}}\right\|_{1}$. By the condition (a) these are finite, and by construction they are monotone non-decreasing functions of $k$. Given a graphex, we then first set each $P_{k, 0}^{\prime}$ to be the set of points with degree greater than $D_{k}$. In this way we have each $P_{k+1,0}^{\prime} \subseteq P_{k, 0}^{\prime}$; however, they may be strictly smaller than the required size. We therefore extend them one by one, starting with $P_{0,0}$, and make sure that we still have each $P_{k+1,0} \subseteq P_{k, 0}$. Taking $\mathbb{W}_{k}$ to be the restrictions, properties (1) and (2) are satisfied.

Next, apply Theorem 5.7 with $\varepsilon=1$ to obtain $N_{0}$ and $\rho_{0}$. Increasing $N_{0}$ (if needed) so that it is of the form $2^{b_{0}}$ for some nonnegative integer $b_{0}$, we then choose $a_{0}$ such that $2^{-a_{0}}<\rho_{0}$. For any graphex, take $\mathscr{P}_{0}$ according to the theorem with $\rho=2^{-a_{0}}$. Keep iterating the theorem, in each step applying Theorem 5.7 with $B=B_{k}, C=C_{k}$, $D=D_{k}, \varepsilon=2^{-k}$, and $\mathscr{P}_{0}=\left(\Omega_{\mathscr{P}_{k-1}} \cup\left(P_{k-1,0} \backslash P_{k, 0}\right), \mathcal{P}_{k} \cup\left\{P_{k-1,0} \backslash P_{k, 0}\right\}\right)$, ensuring in each step that $a_{k} \geq \max \left\{2 k, a_{k-1}+1\right\}, 2^{-a_{k}}<\rho_{k}$, and $b_{k}>b_{k-1}$. 
Lemma 5.9. Every tight sequence of graphexes has a subsequence that is $\delta_{\diamond}$ convergent. More generally, every sequence of signed graphexes obeying the condition (a) from Lemma 5.8 has a $\delta_{\diamond}$-convergent subsequence. If condition (a) holds with one or more of the constants $B, C, D$ not depending on $\varepsilon$, then the subsequential limit inherits the corresponding bound.

During the proof, we will use the following.

Claim. Suppose that $\mathbb{W}=(W, S, I, \boldsymbol{\Omega})$ is a signed graphex, $\mathscr{P}=\left(\Omega_{\mathscr{P}}, \mathcal{P}\right)$ is a finite subspace partition, and $\mathcal{P}_{0} \subseteq \mathcal{P}$. Let $\Omega_{0}=\bigcup_{P \in \mathcal{P}_{0}} P, \mathcal{P}^{\prime}=\mathcal{P} \backslash \mathcal{P}_{0}$, and $\mathscr{P}^{\prime}=\left(\Omega_{\mathscr{P}}^{\prime}, \mathcal{P}^{\prime}\right)$, where $\Omega_{\mathscr{P}^{\prime}}^{\prime}=\Omega_{\mathscr{P}} \backslash \Omega_{0}$. Then $\left.(\mathbb{W} \mathscr{P})\right|_{\Omega^{\prime}}=\left(\left.\mathbb{W}\right|_{\Omega^{\prime}}\right) \mathscr{P}^{\prime}$.

Proof. Note that $\Omega \backslash \Omega_{\mathscr{P}}=\Omega^{\prime} \backslash \Omega_{\mathscr{P} \prime^{\prime}}^{\prime}$ (since $\Omega_{0}$ is disjoint from both). First, we have

$$
\begin{aligned}
I_{\mathscr{P}} & =\frac{1}{2} \int_{(\Omega \backslash \Omega \mathscr{P}) \times\left(\Omega \backslash \Omega_{\mathscr{P}}\right)} W(x, y) d \mu(x) d \mu(y)+\int_{\left(\Omega \backslash \Omega_{\mathscr{P}}\right)} S(x) d \mu(x)+I \\
& =\frac{1}{2} \int_{\left(\Omega^{\prime} \backslash \Omega_{\mathscr{P}^{\prime}}^{\prime}\right) \times\left(\Omega^{\prime} \backslash \Omega_{\mathscr{P}^{\prime}}^{\prime}\right)} W(x, y) d \mu(x) d \mu(y)+\int_{\left(\Omega^{\prime} \backslash \Omega_{\mathscr{P}^{\prime}}^{\prime}\right)} S(x) d \mu(x)+I=I_{\mathscr{P}} .
\end{aligned}
$$

We also have for $x \in \Omega^{\prime}$, if $x \in P_{i}$, then

$$
\begin{aligned}
S_{\mathscr{P}}(x) & =\frac{1}{\mu\left(P_{i}\right)} \int_{P_{i}}\left(S(x)+\int_{\Omega \backslash \Omega_{\mathscr{P}}} W(x, y) d \mu(y)\right) d \mu(x) \\
& =\frac{1}{\mu\left(P_{i}\right)} \int_{P_{i}}\left(S(x)+\int_{\Omega^{\prime} \backslash \Omega_{\mathscr{P}}^{\prime}} W(x, y) d \mu(y)\right) d \mu(x)=S_{\mathscr{P}^{\prime}}(x),
\end{aligned}
$$

and $S_{\mathscr{P}}(x)=0=S_{\mathscr{P}^{\prime}}(x)$ if $x \in \Omega^{\prime} \backslash \Omega_{\mathscr{P}^{\prime}}^{\prime}$. Finally, if $x, y \in \Omega^{\prime}$ and $x \in P_{i}, y \in P_{j}$ with $P_{i}, P_{j} \in \mathcal{P}^{\prime}$, then

$$
W_{\mathscr{P}}(x, y)=\frac{1}{\mu\left(P_{i}\right) \mu\left(P_{j}\right)} \int_{P_{i} \times P_{j}} W\left(x^{\prime}, y^{\prime}\right) d \mu\left(x^{\prime}\right) d \mu\left(y^{\prime}\right)=\left(\left.W\right|_{\Omega^{\prime}}\right)_{s P^{\prime}},
$$

and 0 otherwise.

Proof of Lemma 5.9. Let $\mathbb{W}_{1}, \mathbb{W}_{2}, \ldots, \mathbb{W}_{n}, \ldots$ be a sequence of signed graphexes obeying the condition (a), and let $\widetilde{\mathbb{W}}_{i}$ be obtained from $\mathbb{W}_{i}$ by appending an arbitrary $\sigma$-finite space of infinite measure. Then $\widetilde{\mathbb{W}}_{1}, \widetilde{\mathbb{W}}_{2}, \ldots, \widetilde{\mathbb{W}}_{n}, \ldots$ obeys the condition (a) as well, and arguing as in Remark 4.4, we can assume without loss of generality that $\widetilde{\mathbb{W}}_{1}, \widetilde{\mathbb{W}}_{2}, \ldots, \widetilde{\mathbb{W}}_{n}, \ldots$ are all defined over atomless spaces. We want to show that they have a subsequence that converges to a graphex.

We can take for each $n$ and $k$ sets $\widetilde{P}_{n, k, 0}$ and subspace partitions $\widetilde{\mathscr{P}}_{n, k}$ as in Lemma 5.8, defining in particular $\widetilde{\mathbb{W}}_{n, k}$ as the restriction of $\widetilde{\mathbb{W}}_{n}$ to $\widetilde{\Omega}_{n} \backslash \widetilde{P}_{n, k, 0}$. For $k \geq k_{0}$, we will also define $\widetilde{\mathbb{W}}_{n, k, k_{0}}$ as the restriction of $\left(\widetilde{\mathbb{W}}_{n, k}\right)_{\widetilde{P}_{n, k}}$ to $\widetilde{\Omega}_{n} \backslash \widetilde{P}_{n, k_{0}, 0}$. By the above claim, $\widetilde{\mathbb{W}}_{n, k, k_{0}}=\left(\widetilde{\mathbb{W}}_{n, k_{0}}\right)_{\widetilde{P}_{n, k, k_{0}}}$, where $\widetilde{\mathscr{P}}_{n, k, k_{0}}$ consists of the classes in $\widetilde{P}_{n, k}$ which are subsets of $\widetilde{\Omega}_{n} \backslash \widetilde{P}_{n, k_{0}, 0}$. This implies in particular that $\widetilde{\mathbb{W}}_{n, k, k_{0}}$ is $\left(B_{k_{0}}, C_{k_{0}}, D_{k_{0}}\right)$-bounded.

Furthermore, in view of Remark 3.6, we can replace each $\left(\widetilde{\mathbb{W}}_{n, k}\right)_{\mathscr{P}_{n, k}}$ by an equivalent step function $\mathbb{W}_{n, k}$ over $\mathbb{R}_{+}$, where the first part is $P_{n, k, 0}:=\left[0,2^{-2 k}\right.$ ) (which is disjoint from dsupp $\mathbb{W}_{n, k}$ ), the remaining parts $P_{n, k, i}$ for $i \geq 1$ are of the form $\left[\ell / 2^{a_{k}},(\ell+1) / 2^{a_{k}}\right)$ for some nonnegative integer $\ell$, and we extend $\mathbb{W}_{n, k}$ to zero above $N_{k}+2^{-2 k}$, where $N_{k}=2^{b_{k}}$. 
Let $\mathscr{P}_{k}^{\prime}=\left(\left[2^{-2 k}, N_{k}+2^{-2 k}\right), \mathcal{P}_{k}^{\prime}\right)$, where $\mathcal{P}_{k}^{\prime}$ partitions $\left[2^{-2 k}, N_{k}+2^{-2 k}\right)$ into intervals of length $2^{-a_{k}}$. Note that after this change, the bound (5) from Lemma 5.8 becomes the bound $\delta_{2 \rightarrow 2}\left(\widetilde{\mathbb{W}}_{n, k}, \mathbb{W}_{n, k}\right) \leq 2^{-k}$. In this way, we have mapped the "steps" of each step graphex to $\mathcal{P}_{k}^{\prime}$. For each $k$, the graphex $\mathbb{W}_{n, k}$ then just depends on a finite number of parameters, each bounded. We can then use a diagonalization argument to take a subsequence so that for every $k, \mathbb{W}_{n, k}$ converges to some $\mathbb{W}^{k}$ as $n \rightarrow \infty$, in the sense that $\mathbb{W}^{k}$ is a step graphex with the same parts and each value of the function converges, which also implies that $\left\|\mathbb{W}_{n, k}\right\|_{1} \rightarrow\left\|\mathbb{W}^{k}\right\|_{1}$ and $\mathbb{W}_{n, k} \rightarrow \mathbb{W}^{k}$ in the metric $\delta_{2 \rightarrow 2}$ (since there are a finite number of steps).

Given $k \geq k_{0}$, let $\mathbb{W}^{k, k_{0}}$ be equal to $\mathbb{W}^{k}$ restricted to $\left[2^{-2 k_{0}}, \infty\right)$, let $\mathcal{P}_{k, k_{0}}$ consist of those intervals in $\mathcal{P}_{k}^{\prime}$ which are above $2^{-2 k_{0}}$, and finally, let $\mathscr{P}_{k, k_{0}}=\left(\Omega_{k, k_{0}}, \mathcal{P}_{k, k_{0}}\right)$

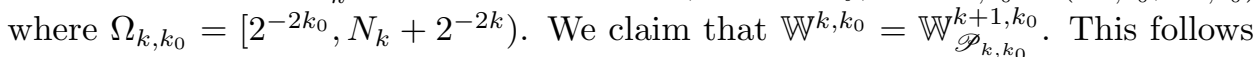
from the fact that for any $n$, if $\mathbb{W}_{n, k, k_{0}}$ is $\mathbb{W}_{n, k}$ restricted to $\left[2^{-2 k_{0}}, \infty\right)$, then $\left(\mathbb{W}_{n, k+1, k_{0}}\right)_{\mathscr{P}_{k, k_{0}}}=\mathbb{W}_{n, k, k_{0}}$, by the above claim.

Next, recalling that $\widetilde{\mathbb{W}}_{n, k, k_{0}}$ is $\left(B_{k_{0}}, C_{k_{0}}, D_{k_{0}}\right)$-bounded for each $n$ and $k$, we have that $\mathbb{W}^{k, k_{0}}$ is $\left(B_{k_{0}}, C_{k_{0}}, D_{k_{0}}\right)$-bounded as well, implying in particular that $\left\|\mathbb{W}^{k, k_{0}}\right\|_{1} \leq C_{k_{0}}$. Also note that if $P_{i}, P_{j} \in \mathcal{P}_{k, k_{0}}$, then

$$
\int_{P_{i} \times P_{j}}\left|W^{k, k_{0}}(x, y)\right| d \mu(x) d \mu(y) \leq \int_{P_{i} \times P_{j}}\left|W^{k+1, k_{0}}(x, y)\right| d \mu(x) d \mu(y) .
$$

Since $W^{k, k_{0}}$ is supported on the union of $P_{i} \times P_{j}$ for all choices of $P_{i}$ and $P_{j}$, this implies that $\left\|W^{k, k_{0}}\right\|_{1}$ cannot decrease as $k$ increases. Together, these observations imply that the limit $\lim _{k^{\prime} \rightarrow \infty}\left\|W^{k^{\prime}, k_{0}}\right\|_{1}$ exists and is at most $C_{k_{0}}$. Given $\varepsilon>0$, we can therefore find $k\left(\varepsilon, k_{0}\right)<\infty$ such that $\lim _{k^{\prime} \rightarrow \infty}\left\|W^{k^{\prime}, k_{0}}\right\|_{1}-\left\|W^{k, k_{0}}\right\|_{1}<\varepsilon$ for all $k \geq k\left(\varepsilon, k_{0}\right)$. Furthermore, because $\Omega_{k, k_{0}}^{2}$ is the support of $W^{k, k_{0}}$,

$$
\int_{\Omega_{k, k_{0}}^{2}}\left|W^{k, k_{0}}(x, y)\right| d \mu(x) d \mu(y) \leq \int_{\Omega_{k, k_{0}}^{2}}\left|W^{k+1, k_{0}}(x, y)\right| d \mu(x) d \mu(y) .
$$

As a consequence, for all $k^{\prime} \geq k\left(\varepsilon, k_{0}\right)$,

$$
\int_{\mathbb{R}_{+}^{2} \backslash \Omega_{k, k_{0}}^{2}}\left|W^{k^{\prime}, k_{0}}(x, y)\right| d \mu(x) d \mu(y)<\varepsilon .
$$

Therefore, the random variables $W^{k, k_{0}}$ are uniformly integrable. By the martingale convergence theorem (applied to each $\left[2^{-2 k_{0}}, 2^{-2 k^{\prime}}+N_{k^{\prime}}\right] \times\left[2^{-2 k_{0}}, 2^{-2 k^{\prime}}+N_{k^{\prime}}\right]$ with $k^{\prime} \geq k_{0}$ ), the graphon part $W^{k, k_{0}}$ of $\mathbb{W}^{k, k_{0}}$ is pointwise convergent almost everywhere to a function $\widetilde{W^{k}} k_{0}$ defined on $\left[2^{-k_{0}}, \infty\right)^{2}$, and it also converges to $\widetilde{W^{k}} k_{0}$ in $L^{1}$. Since $\left\|W^{k, k_{0}}\right\|_{\infty} \leq B_{k_{0}}$, this implies convergence in $L^{2}$, and hence in the kernel metric $\|\cdot\|_{2 \rightarrow 2}$. Furthermore, the graphon marginals converge in $L^{1}$, because

$$
\left\|D_{W^{k, k_{0}}}-D_{\widetilde{W}^{k_{0}}}\right\|_{1} \leq\left\|W^{k, k_{0}}-\widetilde{W}^{k_{0}}\right\|_{1} \underset{k \rightarrow \infty}{\longrightarrow} 0 .
$$

We also have

$$
\int_{P_{i}}\left|D_{\mathbb{W} k, k_{0}}(x)\right| d \mu(x) \leq \int_{P_{i}}\left|D_{\mathbb{W}^{k+1, k_{0}}}(x)\right| d \mu(x)
$$


This implies that $\left\|D_{\mathbb{W} k, k_{0}}\right\|_{1}$ cannot decrease as $k$ increases. Since $\Omega_{k, k_{0}}$ is the support of $D_{\mathbb{W} k, k_{0}}$,

$$
\int_{\Omega_{k, k_{0}}}\left|D_{\mathbb{W}^{k}, k_{0}}(x)\right| d \mu(x) \leq \int_{\Omega_{k, k_{0}}}\left|D_{\mathbb{W}^{k+1, k_{0}}}(x)\right| d \mu(x) .
$$

As before, this implies that the functions $D_{\mathbb{W} k, k_{0}}$ are uniformly integrable and uniformly bounded (by $D_{k_{0}}$ ). We can again use the martingale convergence theorem (applied to each $\left[2^{-2 k_{0}}, 2^{-2 k^{\prime}}+N_{k^{\prime}}\right]$ ) to show that $D_{\mathbb{W} k, k_{0}}$ converges pointwise and in $L^{1}$, and therefore in $L^{2}$, to a function $D_{\mathbb{W}^{k_{0}}}$ taking values in $\left[-D_{k_{0}}, D_{k_{0}}\right]$. Define

$$
\widetilde{S}^{k_{0}}(x)=D_{\widetilde{W}^{k_{0}}}(x)-D_{\widetilde{W}^{k_{0}}}(x) .
$$

Since we also have

$$
S^{k, k_{0}}(x)=D_{\mathbb{W}^{k, k_{0}}}(x)-D_{W^{k, k_{0}}}(x),
$$

and since both terms in the difference converge in $L^{1}, S^{k, k_{0}}$ converges in $L^{1}$ to $\widetilde{S}^{k_{0}}$. By Theorem 3.12 in [24] this implies that some subsequence converges pointwise almost everywhere, showing in particular that in the unsigned case, $\widetilde{S}^{k_{0}} \geq 0$ almost everywhere.

We also have that $\rho:=\rho\left(\mathbb{W}_{k, k_{0}}\right)$ is constant in $k$. Define

$$
I^{k_{0}}=\rho-2 \int \widetilde{S}^{k_{0}}-\int \widetilde{W}^{k_{0}} .
$$

Since for each $k$,

$$
I^{k, k_{0}}=\rho-2 \int S^{k, k_{0}}-\int W^{k, k_{0}}
$$

and since $S^{k, k_{0}}$ and $W^{k, k_{0}}$ converge in $L^{1}$ to $\widetilde{S}^{k_{0}}$ and $\widetilde{W}^{k_{0}}$, respectively, this implies that $I^{k, k_{0}}$ converges to $\widetilde{I}^{k_{0}}$. Because $W^{k, k_{0}}, S^{k, k_{0}}$, and $D_{\mathbb{W}}^{k, k_{0}}$ converge in $L^{1}$ (and hence pointwise almost everywhere on some subsequence), the limit inherits $\left(B_{k_{0}}, C_{k_{0}}, D_{k_{0}}\right)$-boundedness from $\mathbb{W}^{k, k_{0}}$.

Since for each $k, \mathbb{W}^{k, k_{0}+1}$, when restricted to $\left[2^{-2 k_{0}}, \infty\right)$, is equal to $\mathbb{W}^{k, k_{0}}$, and since $\widetilde{\mathbb{W}} k_{0}+1$ and $\widetilde{\mathbb{W}} k_{0}$ are pointwise limits along some subsequence, $\widetilde{\mathbb{W}} k_{0}$ is also the restriction of $\widetilde{\mathbb{W}}^{k_{0}+1}$ to $\left[2^{-2 k_{0}}, \infty\right)$. Therefore, we can define a signed graphex $\mathbb{W}$ on $\mathbb{R}^{+}$as the "union" of the signed graphexes $\widetilde{\mathbb{W}} k_{0}$. Note that the limit $\mathbb{W}$ is locally finite by the fact that $\widetilde{\mathbb{W}} k_{0}$ is $\left(B_{k_{0}}, C_{k_{0}}, D_{k_{0}}\right)$-bounded. This also implies that $\mathbb{W}$ inherits any of these bounds from $\widetilde{\mathbb{W}}^{k_{0}}$ that do not depend on $k_{0}$.

We claim that on the subsequence where $\mathbb{W}_{n, k}$ converges to $\mathbb{W}^{k}$ in $\delta_{2 \rightarrow 2}$ for each $k, \mathbb{W}_{n} \rightarrow \mathbb{W}$ in the weak kernel metric $\delta_{\diamond}$. To see this, we first note that $\widetilde{\mathbb{W}} k_{0}$ is obtained from $\mathbb{W}$ by removing a set of measure $2^{-2 k_{0}}$, and $\mathbb{W}^{k, k_{0}}$ is obtained from $\mathbb{W}^{k}$ by removing a set of the same measure, showing that for any $k_{0}$,

$$
\delta_{\diamond}\left(\mathbb{W}, \mathbb{W}^{k}\right) \leq \max \left\{2^{-k_{0}}, \delta_{2 \rightarrow 2}\left(\widetilde{\mathbb{W}}^{k_{0}}, \mathbb{W}^{k, k_{0}}\right)\right\} .
$$

Given $\varepsilon>0$, choose $k_{0}$ such that $2^{-k_{0}} \leq \varepsilon / 2$. Since $\delta_{2 \rightarrow 2}\left(\mathbb{W}^{k, k_{0}}, \widetilde{\mathbb{W}}^{k_{0}}\right) \rightarrow 0$ for each $k_{0}$ as $k \rightarrow \infty$, this shows that for $k$ large enough, $\delta_{\diamond}\left(\mathbb{W}, \mathbb{W}^{k}\right) \leq \varepsilon / 2$. In a similar way,

$$
\delta_{\diamond}\left(\mathbb{W}_{n}, \mathbb{W}^{k}\right)=\delta_{\diamond}\left(\widetilde{\mathbb{W}}_{n}, \mathbb{W}^{k}\right) \leq \max \left\{2^{-k}, \delta_{2 \rightarrow 2}\left(\widetilde{\mathbb{W}}_{n, k}, \mathbb{W}^{k}\right)\right\} .
$$

Since $\delta_{2 \rightarrow 2}\left(\widetilde{\mathbb{W}}_{n, k}, \mathbb{W}_{n, k}\right) \leq 2^{-k}$ and $\delta_{2 \rightarrow 2}\left(\mathbb{W}_{n, k}, \mathbb{W}^{k}\right) \rightarrow 0$ for each $k$ as $n \rightarrow \infty$, we can first choose $k$ and then $n$ large enough to guarantee that the right side is smaller 
than $\varepsilon / 2$. Combined with the triangle inequality for $\delta_{\diamond}$, this shows that for all $\varepsilon>0$ we can find an $n_{0}$ such that for $n \geq n_{0}$, we have $\delta_{\diamond}\left(\mathbb{W}_{n}, \mathbb{W}\right) \leq \varepsilon$, as claimed.

Remark 5.10. It is not hard to see that a sequence of signed graphexes $\mathbb{W}_{n}=$ $\left(W_{n}, S_{n}, I_{n}, \Omega_{n}\right)$ with $\left\|W_{n}\right\|_{\infty} \leq B$ and $\left\|\mathbb{W}_{n}\right\|_{1} \leq C$ obeys the condition (a) from Lemma 5.8. If $B \leq 1$, this follows by applying Corollary 4.2 (1) to the sequence $\left|\mathbb{W}_{n}\right|$, and for $B>1$ it follows by applying Corollary 4.2 (1) to the sequence $\left(\left|W_{n}\right| / B,\left|S_{n}\right| / B,\left|I_{n}\right| / B, \Omega_{n}\right)$. Choosing a convergent subsequence, it is clear from the last proof that the limiting graphex $\mathbb{W}=(W, S, I, \boldsymbol{\Omega})$ must obey the bound $\|W\|_{\infty} \leq B$. The statement from Remark 2.22 therefore is a direct consequence of Lemma 5.9.

Proof of Theorem 2.21. By Corollary 4.2 (1), any set of graphexes whose $L^{1}$ norms are bounded by $C$ is tight, so by Lemma 5.9 any such sequence of graphexes has a subsequence with a limit $\mathbb{W}$ in the metric $\delta_{\diamond}$. Lemma 5.9 also implies that the limit inherits the bound on the $L^{1}$ norm, and therefore the set of graphexes whose $L^{1}$ norms are bounded by $C$ is compact. The same proof gives the statement of the theorem for $(C, D)$-bounded graphexes.

Suppose now that $\mathbb{W}_{1}, \mathbb{W}_{2}, \ldots, \mathbb{W}_{n}, \ldots$ is a Cauchy sequence in $\delta_{\diamond}$. We first claim that it must be tight. Indeed, for any $\varepsilon>0$, there exists $n$ such that for any $m>n, \delta_{\diamond}\left(\mathbb{W}_{n}, \mathbb{W}_{m}\right)<\varepsilon$. Fix such an $n$ and an $m>n$. By Lemma 3.14, we can then decrease the measures $\mu_{n}, \mu_{m}$ by at most $\varepsilon^{2}$ such that the $\delta_{2 \rightarrow 2}$ distance of the resulting graphexes $\widehat{W}_{n}$ and $\widehat{W}_{m}$ is less than $\varepsilon$. Let $\mathbb{W}_{n}^{\prime}, \mathbb{W}_{m}^{\prime}$ be trivial extensions of the modified graphexes by spaces of infinite measure, and let $\widetilde{\mathbb{W}}_{n}^{\prime}, \widetilde{\mathbb{W}}_{m}^{\prime}$ be pullbacks according to a coupling so that $d_{2 \rightarrow 2}\left(\widetilde{\mathbb{W}}_{n}^{\prime}, \widetilde{\mathbb{W}}_{m}^{\prime}\right)<\varepsilon$, which exists by the definition of $\delta_{2 \rightarrow 2}$. Furthermore, since every finite set of graphexes is tight, we can find $(C, D)$ such that we can remove a set of measure $\varepsilon^{2}$ from $\widehat{\mathbb{W}}_{n}$ to make it $(C, D)$-bounded (independent of $m$ ). If we remove the pullback of this set from the underlying space of $\widetilde{\mathbb{W}}_{n}^{\prime}$ and $\widetilde{\mathbb{W}}_{m}^{\prime}$ and replace them by the restrictions, then the two graphexes will still have $\delta_{2 \rightarrow 2}$ distance at most $2 \varepsilon$ by Lemma 3.13. In particular, this means that $\left\|\widetilde{\mathbb{W}}_{m}^{\prime}\right\|_{1} \leq C+8 \varepsilon^{3}$. We also have

$$
\left\|D_{\widetilde{\mathbb{W}}_{m}^{\prime}}\right\|_{2} \leq\left\|D_{\widetilde{\mathbb{W}}_{n}^{\prime}}\right\|_{2}+\left\|D_{\widetilde{\mathbb{W}}_{m}^{\prime}}-D_{\widetilde{\mathbb{W}}_{n}^{\prime}}\right\|_{2} \leq \sqrt{C D}+4 \varepsilon^{2} \leq 2 \sqrt{C D} .
$$

Therefore, the measure of the points $x$ for which $D_{\widetilde{\mathbb{W}}_{m}^{\prime}}(x)>\sqrt{C D / \varepsilon}$ is at most $4 \varepsilon$. Taking $C^{\prime}=C+\varepsilon$ and $D^{\prime}=\sqrt{C D / \varepsilon}$, we have obtained that for any $m>n$ we can remove a set of measure at most $6 \varepsilon$ so that the remainder is $\left(C^{\prime}, D^{\prime}\right)$-bounded. Since any finite set is tight, and the union of two tight sets is tight, this means that the entire sequence is tight. Therefore, it must have a convergent subsequence that converges to a graphex $\mathbb{W}$. But then because the original sequence was a Cauchy sequence, the entire sequence must converge to $\mathbb{W}$. This proves that the space of graphexes is complete.

The above lemma implies that every tight set is relatively compact, and the fact that any Cauchy sequence must be tight implies that every relatively compact set is tight.

\section{SubGraph COUNTS}

In this section we will prove that convergence in the weak kernel metric implies GP-convergence. The main technical tool for this proof will be the following counting 
lemma, which says that given any $C, D<\infty$, two $(C, D)$-bounded graphexes that are close in kernel metric $\delta_{2 \rightarrow 2}$ must have close subgraph counts.

While this lemma and its corollary are formulated only for unsigned graphexes, we note that both have natural generalizations to signed graphexes. See Remark 6.9 at the end of Section 6.1 below.

Lemma 6.1. Let $F$ be a simple, connected graph with $m$ edges and $n \geq 3$ vertices, and let $C, D<\infty$. Suppose that $\mathbb{W}_{1}$ and $\mathbb{W}_{2}$ are graphexes on the same underlying space $\Omega$, with $\left\|\mathbb{W}_{i}\right\|_{1} \leq C$ and $\left\|D_{\mathbb{W}_{i}}\right\|_{\infty} \leq D$ for $i=1,2$, and let $\varepsilon=\max \left\{\| W_{1}-\right.$ $\left.W_{2}\left\|_{2 \rightarrow 2},\right\| D_{\mathbb{W}_{1}-\mathbb{W}_{2}} \|_{2}\right\}$. Then

$$
\left|t\left(F, \mathbb{W}_{1}\right)-t\left(F, \mathbb{W}_{2}\right)\right| \leq m \varepsilon \widetilde{C} D^{n-3},
$$

where $\widetilde{C}=\max \{C, \sqrt{C D}\}$.

Corollary 6.2. Suppose $\mathbb{W}_{n}$, $\mathbb{W}$ have uniformly bounded marginals. If

$$
\delta_{2 \rightarrow 2}\left(\mathbb{W}_{n}, \mathbb{W}\right) \rightarrow 0,
$$

then for any finite graph $F$ with no isolated vertices, $t\left(F, \mathbb{W}_{n}\right)$ converges to $t(F, \mathbb{W})$.

Proof. By the definition of $\delta_{2 \rightarrow 2}$, if $F$ is an edge, then $t\left(F, \mathbb{W}_{n}\right) \rightarrow t(F, \mathbb{W})$. This implies in particular that $\|\mathbb{W}\|_{1}$ and $\left\|\mathbb{W}_{n}\right\|_{1}$ are uniformly bounded. By Lemma 6.1, it follows that $t\left(F, \mathbb{W}_{n}\right) \rightarrow t(F, \mathbb{W})$ for any connected graph $F$. Since homomorphism densities factor over connected components of the graph $F$, this means that if $F$ is a finite graph without isolated vertices, then $t\left(F, \mathbb{W}_{n}\right) \rightarrow t(F, \mathbb{W})$.

In a addition to the above counting lemma, we will need to show that convergence of subgraph counts implies GP-convergence. Thinking of the subgraph counts as the moments of a graphex, this result is similar to standard moment theorems for random variables that show that under suitable growth conditions, the distribution of a random variable is determined by its moments.

Theorem 6.3. Assume that the marginals of $\mathbb{W}_{n}$ and $\mathbb{W}$ are bounded by some finite constant $D$. Then the following are equivalent:

(1) $G_{T}\left(\mathbb{W}_{n}\right) \rightarrow G_{T}(\mathbb{W})$ in distribution for every $T$.

(2) $G_{T}\left(\mathbb{W}_{n}\right) \rightarrow G_{T}(\mathbb{W})$ in distribution for some $T$.

(3) For every graph $F$ with no isolated vertices, $t\left(F, \mathbb{W}_{n}\right) \rightarrow t(F, \mathbb{W})$.

(4) For every connected graph $F, t\left(F, \mathbb{W}_{n}\right) \rightarrow t(F, \mathbb{W})$.

We will prove the counting lemma in Subsection 6.1 below, and Theorem 6.3 in Subsection 6.2. In the final subsection, Subsection 6.3, we use these results to first

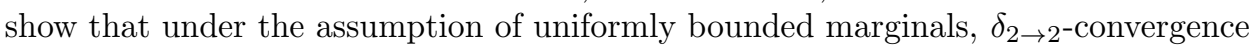
implies GP-convergence (Theorem 6.16 below). With the help of the results about tightness established in Section 4, this in turn allows us to show that without any assumption on the marginals, $\delta_{\diamond}$-convergence implies GP-convergence (Theorem 6.17 below).

6.1. Proof of the counting lemma. In order to prove the counting lemma, it will be convenient to consider several variants of the homomorphism densities. For these variants, it will be natural to consider signed graphexes, since we will need to consider differences of graphexes for the proof of the counting lemma anyway. Note that our proof of the counting lemma can easily be generalized to signed graphons; see Remark 6.9 below. 
Definition 6.4. Suppose we have a connected multigraph $F$, and signed graphexes $\mathbb{W}_{e}$ assigned to each edge $e \in E(F)$ (refer to this vector of graphexes as $\mathbb{W}_{F}$ ), each with the same feature space $\Omega$. Let $V_{\geq 2}$ be the set of vertices with degree at least 2 . If $V_{\geq 2}$ is nonempty (i.e., $F$ does not consist of a single edge), then we define

$$
\begin{aligned}
& t\left(F, \mathbb{W}_{F}\right)=\int_{\Omega^{V \geq 2}} d z_{V_{\geq 2}} \prod_{\{v, w\} \in E\left(F\left(V_{\geq 2}\right)\right)} W_{\{v, w\}}\left(z_{v}, z_{w}\right) \\
& \cdot \prod_{v \in V_{\geq 2}} \prod_{\substack{w \in V \backslash V_{\geq 2}: \\
\{v, w\} \in E(F)}} D_{\mathbb{W}_{\{v, w\}}}\left(z_{v}\right) .
\end{aligned}
$$

If $F$ consists of just a single edge $f$, then

$$
t\left(F, \mathbb{W}_{F}\right)=\rho\left(\mathbb{W}_{f}\right),
$$

with $\rho\left(\mathbb{W}_{f}\right)$ as in $(2.2)$. Note that for signed graphexes $t\left(F, \mathbb{W}_{F}\right)$ is in general only well defined if the integrals are absolutely convergent, a condition which can, e.g., be guaranteed by requiring that $t\left(F, \mathbb{W}_{F}^{\text {abs }}\right)<\infty$, where $\mathbb{W}_{F}^{\text {abs }}$ is obtained from $\mathbb{W}_{F}$ by replacing the graphexes $\mathbb{W}_{f}$ by $\left|\mathbb{W}_{f}\right|$.

Note that $t\left(F, \mathbb{W}_{F}\right)$ is a multilinear function of the signed graphexes $\mathbb{W}_{e}$ in $\mathbb{W}_{F}$. If each $\mathbb{W}_{e}$ is equal to some fixed $\mathbb{W}$, then this is just the previous definition.

We also define a conditional density where we fix the image of a single vertex.

Definition 6.5. Suppose we have a connected multigraph $F$ with a labeled vertex $v_{0}$, more than one edge, and signed graphexes $\mathbb{W}_{e}$ assigned to each edge as before. Let $x \in \Omega$, and define $z_{v_{0}}=x$. Take, furthermore, $V_{\geq 2}^{\prime}=V_{\geq 2} \backslash\left\{v_{0}\right\}$ and $\widetilde{V}_{\geq 2}=V_{\geq 2} \cup\left\{v_{0}\right\}$. Then we define $t_{x}\left(F, \mathbb{W}_{F}\right)$ to be

$$
\int_{\Omega^{V_{\geq 2}^{\prime}}} d z_{V_{\geq 2}^{\prime}} \prod_{\{v, w\} \in E\left(F\left(\widetilde{V}_{\geq 2}\right)\right)} W_{\{v, w\}}\left(z_{v}, z_{w}\right) \prod_{v \in V_{\geq 2}} \prod_{\substack{w \in V \backslash\left(\widetilde{V}_{\geq 2}\right): \\\{v, w\} \in E(F)}} D_{\mathbb{W}_{\{v, w\}}}\left(z_{v}\right) .
$$

If $F$ consists of just a single edge $f$ adjacent to $v_{0}$, then we set $t_{x}(F, \mathbb{W})=D_{\mathbb{W}}(x)$. Again, for signed graphexes, this is in general only well defined if the integrals are absolutely convergent.

Note that if $v_{0} \in V_{>2}$, then $t_{x}\left(F, \mathbb{W}_{F}\right)$ is obtained from $t\left(F, \mathbb{W}_{F}\right)$ by simply fixing the feature corresponding to $v_{0}$ to be $x$, implying in particular that $\int t_{x}\left(F, \mathbb{W}_{F}\right) d x=$ $t\left(F, \mathbb{W}_{F}\right)$ (assuming the integrals defining these are absolutely convergent). If $v_{0} \notin V_{\geq 2}$, i.e., if $v_{0}$ has degree 1 , and if $F$ has more than one edge, then the situation is slightly more complicated, since the "feature" of the image of $v_{0}$ could be either an element of $\Omega$, or the special value $\infty$, interpreted earlier as the feature of the leaves of the star part of a graphon process. With this reinterpretation, $t_{x}\left(F, \mathbb{W}_{F}\right)$ is still obtained from $t\left(F, \mathbb{W}_{F}\right)$ by fixing the feature corresponding to $v_{0}$ to be $x$, but the integral $\int t_{x}\left(F, \mathbb{W}_{F}\right) d x$ now misses the contribution of $x=\infty$, and hence is in general only bounded above by $t\left(F, \mathbb{W}_{F}\right)$. It is, however, equal to $t\left(F, \mathbb{W}_{F}^{\prime}\right)$, where $\mathbb{W}_{F}^{\prime}$ is obtained from $\mathbb{W}_{F}$ by setting the star part of the graphex corresponding to the edge containing $v_{0}$ to 0 .

Lemma 6.6. Suppose $F$ is a connected multigraph with no loops and a labeled vertex $v_{0}, T$ is a spanning tree, and $\mathbb{W}_{e}$ is a signed graphex corresponding to each 
edge e, each with the same feature space $\Omega$. Let $f \in T$ be an edge adjacent to $v_{0}$, and $x \in \Omega$. Then

$$
\left|t_{x}\left(F, \mathbb{W}_{F}\right)\right| \leq D_{\left|\mathbb{W}_{f}\right|}(x) \prod_{e \in T \backslash f}\left\|D_{\left|\mathbb{W}_{e}\right|}\right\|_{\infty} \prod_{e \in E(F) \backslash T}\left\|W_{e}\right\|_{\infty} .
$$

Proof. Replacing all signed graphexes $\mathbb{W}_{e}$ by the non-negative versions $\left|\mathbb{W}_{e}\right|$, and noting that $\left|t_{x}\left(F, \mathbb{W}_{F}\right)\right| \leq t_{x}\left(F, \mathbb{W}_{F}^{\mathrm{abs}}\right)$, we may without loss of generality assume that $W_{e}$ and $S_{e}$ are non-negative.

Next, assume that $F$ is a tree, i.e., $F=T$. We then prove the claim by induction on the number of edges. If $F$ consists of a single edge, then by definition $t_{x}(F, \mathbb{W})=D_{\mathbb{W}}(x)$, which is exactly the bound in the lemma. Otherwise, we can find an edge $\{v, w\}$ not equal to $f$, such that $v$ has degree at least two, and $w$ has degree 1 and is different from $v_{0}$. The edge $\{v, w\}$ then contributes $D_{\mathbb{W}_{\{v, w\}}}\left(z_{v}\right)$ to the second product in the integral representing $t_{x}\left(F, \mathbb{W}_{F}\right)$. For each $z_{v}$, this is at most $\left\|D_{\mathbb{W}_{\{v, w\}}}\right\|_{\infty}$. Taking a factor $\left\|D_{\mathbb{W}_{\{v, w\}}}\right\|_{\infty}$ out of the integral, and defining $F^{\prime}$ to be the restriction of $F$ to $V(F) \backslash\{w\}$, we therefore have that

$$
t_{x}\left(F, \mathbb{W}_{F}\right) \leq\left\|D_{\mathbb{W}_{\{v, w\}}}\right\|_{\infty} t_{x}\left(F^{\prime}, \mathbb{W}_{F^{\prime}}\right) .
$$

Note that this bound is actually weaker than necessary if the vertex $v$ becomes a vertex of degree one in $F^{\prime}$, in which case we could have obtained a contribution of $D_{W_{\left\{v, v^{\prime}\right\}}}\left(z_{v^{\prime}}\right)$ for its neighbor $v^{\prime}$ instead of the contribution $D_{\mathbb{W}_{\left\{v, v^{\prime}\right\}}}\left(z_{v^{\prime}}\right)$ implicit in the above bound.

Suppose now that $F$ has edges outside $T$. Let $\{v, w\}$ be such an edge. Note that both $v$ and $w$ must be in $V_{\geq 2}$. Therefore, this edge contributes $W_{\{v, w\}}\left(z_{v}, z_{w}\right)$ to the product, which is at most $\left\|W_{\{v, w\}}\right\|_{\infty}$. We can therefore conclude the lemma by induction on the number of edges of $F$ outside $T$.

Lemma 6.7. Suppose $F$ is a connected multigraph with no loops, and we have a signed graphex $\mathbb{W}_{e}$ corresponding to each edge $e \in F$. Let $T$ be any spanning tree in $F$, and $f \in T$. Then

$$
\left|t\left(F, \mathbb{W}_{F}\right)\right| \leq\left\|\mathbb{W}_{f}\right\|_{1} \prod_{e \in T \backslash f}\left\|D_{\left|\mathbb{W}_{e}\right|}\right\|_{\infty} \prod_{e \in E(F) \backslash T}\left\|W_{e}\right\|_{\infty} .
$$

Proof. If $F$ consists of a single edge $f$, then $\left|t\left(F, \mathbb{W}_{F}\right)\right|=\left|\rho\left(\mathbb{W}_{f}\right)\right| \leq\left\|\mathbb{W}_{f}\right\|_{1}$ by definition, and if $F$ has more than one edge, then

$$
\begin{aligned}
\left|t\left(F, \mathbb{W}_{F}\right)\right| & \leq \int_{\Omega}\left|t_{x}\left(F, \mathbb{W}_{F}\right)\right| d \mu(x) \\
& \leq \int_{\Omega} D_{\left|\mathbb{W}_{f}\right|}(x) \prod_{e \in T \backslash f}\left\|D_{\left|\mathbb{W}_{e}\right|}\right\|_{\infty} \prod_{e \in E(F) \backslash T}\left\|W_{e}\right\|_{\infty} d \mu(x) \\
& \leq\left\|\mathbb{W}_{f}\right\|_{1} \prod_{e \in T \backslash f}\left\|D_{\left|\mathbb{W}_{e}\right|}\right\|_{\infty} \prod_{e \in E(F) \backslash T}\left\|W_{e}\right\|_{\infty} .
\end{aligned}
$$

Proof of Lemma 6.1. Let $f=\{u, v\}$ be an edge in $F$, and let $\mathbb{W}_{F, f}$ be a vector of graphexes where we assign one of $\mathbb{W}_{1}$ or $\mathbb{W}_{2}$ to each edge $e \neq f$, and $\left(W_{1}-W_{2}, S_{1}-\right.$ $\left.S_{2}, 0, \boldsymbol{\Omega}\right)$ to $f$ (since $F$ is a connected graph with at least 2 edges, the dust parts of $\mathbb{W}_{1}$ and $\mathbb{W}_{2}$ don't contribute to $t\left(F, \mathbb{W}_{1}\right)-t\left(F, \mathbb{W}_{2}\right)$ and can be set to 0$)$. We would like to bound $\left|t\left(F, \mathbb{W}_{F, f}\right)\right|$.

First, assume both endpoints of $f$ have degree at least 2. Let the components of $F$ restricted to $V(F) \backslash\{u, v\}$ be $C_{1}, C_{2}, \ldots, C_{k}$, with corresponding vertex sets 
$V_{1}, \ldots, V_{k}$. There can be three types of components: those with at least one edge to $u$ but none to $v$, those with at least one edge to $v$ but none to $u$, and those with at least one edge to both. Let $\mathcal{C}_{u}$ be the set of components connected to $u, \mathcal{C}_{v}$ the set of those connected to $v$, and $\mathcal{C}_{u v}$ the set connected to both. For each $i \in \mathcal{C}_{u}$, let $F_{i}$ be the labeled graph where we add $u$ back to $C_{i}$ as the labeled vertex, and for each $i \in \mathcal{C}_{y}$, let $F_{i}$ be the labeled graph where we add $v$ back to $C_{i}$ as the labeled vertex. Furthermore, for each $i \in \mathcal{C}_{u} \cup \mathcal{C}_{v}$, choose an additional vertex $v_{i} \in V_{i}$ such that $v_{i}$ is incident to an edge in $F_{i}$. Let $V_{u v}$ consist of vertices that belong to a component in $\mathcal{C}_{u v}$.

Given a set of vertices $U$, let $U^{\prime}$ be the set of vertices in $U$ that have degree at least 2 in $F$, and let

$$
W_{F, U, u}\left(z_{u}, z_{U^{\prime}}\right)=\prod_{\substack{w \in U^{\prime} \\\{u, w\} \in E(F)}} W_{\{u, w\}}\left(z_{u}, z_{w}\right)
$$

and

$$
W_{F, U, v}\left(z_{v}, z_{U^{\prime}}\right)=\prod_{\substack{w \in U^{\prime} \\\{v, w\} \in E(F)}} W_{\{v, w\}}\left(z_{v}, z_{w}\right) .
$$

Let us also use the notation

$$
\mathbb{W}_{F, U}\left(z_{U^{\prime}}\right)=\prod_{\left\{w, w^{\prime}\right\} \in E\left(F\left(U^{\prime}\right)\right)} W_{\left\{w, w^{\prime}\right\}}\left(z_{w}, z_{w^{\prime}}\right) \prod_{w \in U^{\prime}} \prod_{\substack{w^{\prime} \in U \backslash U^{\prime} \\\left\{w, w^{\prime}\right\} \in E(F)}} D_{\mathbb{W}_{\left\{w, w^{\prime}\right\}}}\left(z_{w}\right) .
$$

Observe that if a vertex in $V_{u v}$ is adjacent to $u$ or $v$, it must be in $V_{u v}^{\prime}$. We therefore express $t\left(F, \mathbb{W}_{F, f}\right)$ as

$$
\begin{gathered}
\int_{\Omega^{V_{u v}^{\prime}}} \mathbb{W}_{F, V_{u v}}\left(z_{V_{u v}^{\prime}}\right) \int_{\Omega^{2}}\left(W_{F, V_{u v}, v}\left(z_{v}, z_{V_{u v}^{\prime}}\right) W_{F, V_{u v}, u}\left(z_{u}, z_{V_{u v}^{\prime}}\right) W_{f}\left(z_{u}, z_{v}\right)\right. \\
\left.\prod_{i \in \mathcal{C}_{u}} t_{z_{u}}\left(F_{i}, \mathbb{W}_{F, f}\right) \prod_{i \in \mathcal{C}_{v}} t_{z_{v}}\left(F_{i}, \mathbb{W}_{F, f}\right)\right)
\end{gathered}
$$

and bound the inner integral by

$$
\begin{aligned}
& \left\|W_{F, V_{u v}, u}\left(\cdot, z_{V_{u v}^{\prime}}\right) \prod_{i \in \mathcal{C}_{u}} t .\left(F_{i}, \mathbb{W}_{F, f}\right)\right\|_{2} \\
& \left\|W_{F, V_{u v}, v}\left(\cdot, z_{V_{u v}^{\prime}}\right) \prod_{i \in \mathcal{C}_{v}} t .\left(F_{i}, \mathbb{W}_{F, f}\right)\right\|_{2}\left\|W_{f}\right\|_{2 \rightarrow 2} \\
& \leq\left\|W_{F, V_{u v}, u}\left(\cdot, z_{V_{u v}^{\prime}}\right) \prod_{i \in \mathcal{C}_{u}} D_{\mathbb{W}_{u v_{i}}}\right\|_{2}\left\|W_{F, V_{u v}, v}\left(\cdot, z_{V_{u v}^{\prime}}\right) \prod_{i \in \mathcal{C}_{v}} D_{\mathbb{W}_{v v_{i}}}\right\|_{2}\left\|W_{f}\right\|_{2 \rightarrow 2} \prod_{i \in \mathcal{C}_{v} \cup \mathcal{C}_{u}} D^{\left|V_{i}\right|-1},
\end{aligned}
$$

where in the last step we used Lemma 6.6 and the fact that the number of edges in a spanning tree for $F_{i}$ is $\left|V\left(F_{i}\right)\right|-1=\left|V_{i}\right|$. Inserting this bound into the outer 
integral, an application of the Cauchy-Schwartz inequality then gives the bound

$$
\begin{aligned}
& t\left(F, \mathbb{W}_{F, f}\right) \leq\left\|W_{f}\right\|_{2 \rightarrow 2} \sqrt{\int_{\Omega^{V_{u v}^{\prime}}} \mathbb{W}_{F, V_{u v}}\left(z_{V_{u v}^{\prime}}\right)\left\|W_{F, V_{u v}, u}\left(\cdot, z_{V_{u v}^{\prime}}\right) \prod_{i \in \mathcal{C}_{u}} D_{\mathbb{W}_{u v_{i}}}\right\|_{2}^{2}} \\
& \cdot \sqrt{\int_{\Omega^{V_{u v}^{\prime}}} \mathbb{W}_{F, V_{u v}}\left(z_{V_{u v}^{\prime}}\right)\left\|W_{F, V_{u v}, v}\left(\cdot, z_{V_{u v}^{\prime}}\right) \prod_{i \in \mathcal{C}_{v}} D_{\mathbb{W}_{v v_{i}}}\right\|_{2}^{2}} \prod_{i \in \mathcal{C}_{v} \cup \mathcal{C}_{u}} D^{\left|V_{i}\right|-1}
\end{aligned}
$$

We claim that the expressions under the square roots can be written as $t\left(F_{u}^{\prime}, \mathbb{W}_{F, f}\right)$ and $t\left(F_{v}^{\prime}, \mathbb{W}_{F, f}\right)$ for some suitable multigraphs $F_{u}^{\prime}$ and $F_{v}^{\prime}$. Indeed, starting from $F\left(V_{u v} \cup\{u\}\right)$, we first duplicate every edge in this graph that joins $u$ to some vertex in $V_{u v}$, keeping the edges between vertices in $V_{u v}$ as simple edges. The graph $F_{u}^{\prime}$ is obtained from this graph by adding two more edges for each component $i \in \mathcal{C}_{u}$ : the edge $u v_{i}$, and a second edge $u v_{i}^{\prime}$, with $v_{i}^{\prime}$ being a new vertex we should think of as a twin of $v_{i}$ (in $F_{u}^{\prime}$, they both have degree one and are connected to $u$ ). This gives a connected multigraph on $V_{u}^{\prime}=V\left(F_{u}^{\prime}\right)$ with $\left|V_{u v}\right|+1+2 k_{u}$ many vertices and $\left|E\left(F\left(V_{u v}\right)\right)\right|+2 d_{u}+2 k_{u}$ many edges where $d_{u}$ is the number of vertices $v^{\prime} \in V_{u v}$ such that $u v^{\prime}$ is an edge in $F$, and $k_{u}=\left|\mathcal{C}_{u}\right|$. Define $F_{v}^{\prime}$ (as well as $d_{v}$ and $k_{v}$ ) analogously. We then can reexpress the above bound as

$$
\begin{aligned}
t\left(F, W_{F, f}\right) & \leq\left\|W_{f}\right\|_{2 \rightarrow 2} \sqrt{t\left(F_{u}^{\prime}, \mathbb{W}_{F, f}\right) t\left(F_{v}^{\prime}, \mathbb{W}_{F, f}\right)} \prod_{i \in \mathcal{C}_{v} \cup \mathcal{C}_{u}} D^{\left|V_{i}\right|-1} \\
& \leq\left\|W_{f}\right\|_{2 \rightarrow 2} C D^{\left|V_{u v}\right|+k_{u}+k_{v}-1} \prod_{i \in \mathcal{C}_{v} \cup \mathcal{C}_{u}} D^{\left|V_{i}\right|-1} \\
& =\left\|W_{f}\right\|_{2 \rightarrow 2} C D^{n-3} .
\end{aligned}
$$

If $f$ has one endpoint with degree 1 , and the other endpoint with degree at least 2 , then let $v$ be the endpoint with degree at least 2 , and let $u^{\prime} \neq u$ be a neighbor of $v$. Let $\mathbb{W}_{F-f}$ be the graphex assignment restricted to the edges in $F-f$. Then

$$
\begin{aligned}
\left|t\left(F, \mathbb{W}_{F, f}\right)\right| & =\left|\int_{\Omega} t_{x_{v}}\left(F-f, \mathbb{W}_{F-f}\right) D_{\mathbb{W}_{1}-\mathbb{W}_{2}}\left(x_{v}\right) d \mu\left(x_{v}\right)\right| \\
& \leq\left\|D_{\mathbb{W}_{1}-\mathbb{W}_{2}}\right\|_{2}\left\|t .\left(F-f, \mathbb{W}_{F-f}\right)\right\|_{2} \\
& \leq\left\|D_{\mathbb{W}_{1}-\mathbb{W}_{2}}\right\|_{2}\left\|D_{\mathbb{W}_{v u^{\prime}}}\right\|_{2} D^{n-3} \leq \sqrt{C D} D^{n-3}\left\|D_{\mathbb{W}_{1}-\mathbb{W}_{2}}\right\|_{2} .
\end{aligned}
$$

Now, let $e_{1}, e_{2}, \ldots, e_{m}$ be the edges of $F$. Let $\mathbb{W}_{F, i}$ be the vector of graphexes where we assign $\mathbb{W}_{1}-\mathbb{W}_{2}$ to $e_{i}, \mathbb{W}_{1}$ to $e_{j}$ with $j<i$, and $\mathbb{W}_{2}$ to $e_{j}$ with $j>i$. Then,

$$
\left|t\left(F, \mathbb{W}_{1}\right)-t\left(F, \mathbb{W}_{2}\right)\right| \leq \sum_{i=1}^{m}\left|t\left(F, \mathbb{W}_{F, i}\right)\right| \leq m \varepsilon \widetilde{C} D^{n-3}
$$

Remark 6.8. It is instructive to note that the bound in Lemma 6.1 can be tightened to give the constant

$$
\widetilde{C}=\max _{i} \max \left\{\left\|W_{i}\right\|_{2},\left\|D_{\mathbb{W}_{i}}\right\|_{2}\right\}
$$

instead of the constant $\widetilde{C}=\max \{C, \sqrt{C D}\}$. To see this, we first note that near the end of the proof, we bounded $\left\|D_{\mathbb{W}_{v u^{\prime}}}\right\|_{2}$ by $\sqrt{C D}$, even though it is possible that the first term is finite while the second is infinite. In a similar way, bounding the integral representing $t\left(F_{u}^{\prime}, \mathbb{W}_{F, f}\right)$ and $t\left(F_{v}^{\prime}, \mathbb{W}_{F, f}\right)$ with the help of Lemma 6.7 is suboptimal. Indeed, the multi-graph $F_{v}^{\prime}$ always contains at least one double edge, 
or contain at least one edge $u v_{i}$ and its twin $u v_{i}^{\prime}$, with both $v_{i}$ and $v_{i}^{\prime}$ having degree one. In the first case, Lemma 6.7 can be improved to extract a factor $\left\|W_{f}\right\|_{2}$ instead of a factor $\left\|\mathbb{W}_{f}\right\|_{1}$, in the second it can be improved to extract a factor $\left\|D_{\mathbb{W}_{f}}\right\|_{2}$. Inserted into the proof of Lemma 6.1, this gives the claimed improvement.

Remark 6.9. As the reader can easily verify, the above proof immediately generalizes to signed graphexes, showing that Lemma 6.1 holds for $(B, C, D)$ bounded graphexes, provided we include a factor of $B^{m-(n-1)}$ on the right side. As a consequence, Corollary 6.2 holds for sequences of $(B, C, D)$-bounded graphexes that converge in the kernel distance $\delta_{2 \rightarrow 2}$.

6.2. GP-convergence and subgraph counts. In this subsection, we prove Theorem 6.3. We start by establishing the following theorem.

Theorem 6.10. Let $\mathbb{G}$ and $\mathbb{G}_{n}$, for $n \geq 1$, be random finite graphs with no isolated vertices, and let $X$ and $X_{n}$, for $n \geq 1$, be the random variables that correspond to the number of vertices in $\mathbb{G}$ and $\mathbb{G}_{n}$, respectively. If, for every $t>0, \mathbb{E}\left[e^{t X}\right]$ and $\mathbb{E}\left[e^{t X_{n}}\right]$ are finite and uniformly bounded, then the following are equivalent:

(1) For any graph $G$, the probability that $\mathbb{G}_{n}$ is isomorphic to $G$ converges to the probability that $\mathbb{G}$ is isomorphic to $G$.

(2) For every graph $F, \mathbb{E}\left[\operatorname{inj}\left(F, \mathbb{G}_{n}\right)\right] \rightarrow \mathbb{E}[\operatorname{inj}(F, \mathbb{G})]$.

(3) For every graph $F$ with no isolated vertices, $\mathbb{E}\left[\operatorname{inj}\left(F, \mathbb{G}_{n}\right)\right] \rightarrow \mathbb{E}[\operatorname{inj}(F, \mathbb{G})]$.

To prove the theorem, we will first establish a couple of lemmas. As a preparation, note that if $\mathbb{E}\left[e^{t X}\right] \leq C$, then for any graph $F$ on $k$ vertices,

$$
\mathbb{E}[\operatorname{inj}(F, \mathbb{G})] \leq \sum_{n=0}^{\infty} P(X=n) n^{k} \leq \sum_{n=0}^{\infty} P(X=n) \frac{k !}{t^{k}} e^{t n}=\frac{k !}{t^{k}} \mathbb{E}\left[e^{t X}\right] \leq C^{\prime} .
$$

Here we use the fact that $e^{t n} \geq \frac{(t n)^{k}}{k !}$. The same bound holds for $\mathbb{G}_{n}$. In other words, for any graph $F$ the values $\mathbb{E}\left[\operatorname{inj}\left(F, \mathbb{G}_{n}\right)\right]$ and $\mathbb{E}[\operatorname{inj}(F, \mathbb{G})]$ are bounded uniformly in $n$.

Our first lemma roughly says that if a random graph model does not have isolated vertices, and the number of vertices is not too large with high probability, then the expected number of counts of finite graphs without isolated vertices determines the expected number of counts of all graphs.

Lemma 6.11. Suppose we have two random finite graphs with no isolated vertices, $\mathbb{G}$ and $\mathbb{G}^{\prime}$. Suppose that for every finite graph $F$ with no isolated vertices, $\mathbb{E}[\operatorname{inj}(F, \mathbb{G})]=$ $\mathbb{E}\left[\operatorname{inj}\left(F, \mathbb{G}^{\prime}\right)\right]$. Let $X$ be the random variable that gives the number of vertices in $\mathbb{G}$, and suppose that for every $t, \mathbb{E}\left[e^{t X}\right]$ is finite. Then $\mathbb{E}[\operatorname{inj}(F, \mathbb{G})]$ and $\mathbb{E}\left[\operatorname{inj}\left(F, \mathbb{G}^{\prime}\right)\right]$ are equal for every finite graph $F$.

Proof. We prove this by induction on the number of isolated vertices in $F$. If $F$ has zero isolated vertices, the claim is true by the assumptions of the lemma. Otherwise, let $F$ consist of $F^{\prime}$ plus an isolated vertex $w_{0}$. For every $k$, and graph $G$, let $\operatorname{inj}^{*}\left(F^{\prime}, k, G\right)$ be equal to the number of ways we can take an injective image of $F^{\prime}$ in $G$, take a vertex $v_{0}$ not in the image of $F^{\prime}$, and take a $k$-term sequence of distinct neighbors of $v_{0}$ (which may or may not be in the image of $F^{\prime}$ ). If $G$ has no isolated vertices, then

$$
\operatorname{inj}(F, G)=\sum_{k=1}^{\infty} \frac{(-1)^{k-1}}{k !} \operatorname{inj}^{*}\left(F^{\prime}, k, G\right) .
$$


This follows from the fact that for each injective copy of $F$, if $v_{0}$ is the image of $w_{0}$, then this contributes $\left(d\left(v_{0}\right)\right)_{k}$ to $\operatorname{inj}^{*}\left(F^{\prime}, k, G\right)$. Since $G$ is finite and has no isolated vertices, we must have $0<d\left(v_{0}\right)<\infty$; therefore,

$$
\sum_{k=1}^{\infty} \frac{(-1)^{k-1}}{k !}\left(d\left(v_{0}\right)\right)_{k}=\sum_{k=1}^{d\left(v_{0}\right)}(-1)^{k-1}\left(\begin{array}{c}
d\left(v_{0}\right) \\
k
\end{array}\right)=1
$$

Next, we claim that

$$
\mathbb{E}[\operatorname{inj}(F, \mathbb{G})]=\sum_{k=1}^{\infty} \frac{(-1)^{k-1}}{k !} \mathbb{E}\left[\operatorname{inj}^{*}\left(F^{\prime}, k, \mathbb{G}\right)\right]
$$

To show this, it suffices to show that

$$
\sum_{k=1}^{\infty} \frac{\mathbb{E}\left[\operatorname{inj}^{*}\left(F^{\prime}, k, \mathbb{G}\right)\right]}{k !}<\infty
$$

Fix $k$. Then $\operatorname{inj}^{*}\left(F^{\prime}, k, G\right)$ is the sum of terms of the form $\operatorname{inj}\left(F^{\prime \prime}, G\right)$. The terms are obtained as follows. Let $L=V\left(F^{\prime}\right) \cup z_{0}$, where $z_{0}$ is disjoint from $V\left(F^{\prime}\right)$. Let $\ell=\left(\ell_{1}, \ell_{2}, \ldots, \ell_{k}\right)$ be a sequence of $k$ elements of $L$. Suppose further that any vertex in $V\left(F^{\prime}\right)$ appears at most once in $\ell$. Then we define $F^{\prime \prime}(\ell)$ by taking a copy of $F^{\prime}$, a disjoint vertex $w_{0}$, for each $\ell_{i} \in V\left(F^{\prime}\right)$, we add an edge from $w_{0}$ to $\ell_{i}$, for each other $\ell_{i}$ we add an edge going to a new vertex. For example, if $\ell=\left(z_{0}, z_{0}, z_{0}, \ldots, z_{0}\right)$, then $F^{\prime \prime}(\ell)$ is the disjoint union of $F^{\prime}$ and a star with $k$ edges. It is then not difficult to see that

$$
\operatorname{inj}^{*}\left(F^{\prime}, k, G\right)=\sum_{\ell} \operatorname{inj}\left(F^{\prime \prime}(\ell), G\right) .
$$

Let $a=|L|$. For each $k$, the number of such sequences is at most $a^{k}$. Furthermore, for each such $F^{\prime \prime}(\ell)$, the number of vertices is at most $a+k$. Thus,

$$
\operatorname{inj}^{*}\left(F^{\prime}, k, G\right) \leq a^{k}(|V(G)|)_{(a+k)} .
$$

Therefore, recalling that $X=|\mathbb{G}|$, we have that

$$
\begin{aligned}
& \sum_{k=1}^{\infty} \frac{\mathbb{E}\left[\operatorname{inj}^{*}\left(F^{\prime}, k, \mathbb{G}\right)\right]}{k !} \leq \sum_{k=1}^{\infty} \frac{a^{k} \mathbb{E}\left[(X)_{(a+k)}\right]}{k !} \\
& =\sum_{k=1}^{\infty} \mathbb{E}\left[a^{k} X_{(a)}\left(\begin{array}{c}
X-a \\
k
\end{array}\right)\right]=\mathbb{E}\left[X_{(a)}(a+1)^{X-a}\right]<\infty
\end{aligned}
$$

Finally, we claim that $\mathbb{E}\left[\operatorname{inj}^{*}\left(F^{\prime}, k, \mathbb{G}\right)\right]=\mathbb{E}\left[\operatorname{inj}^{*}\left(F^{\prime}, k, \mathbb{G}^{\prime}\right)\right]$ for every $k$. This follows from the fact that the graphs $F^{\prime \prime}(\ell)$ above each have fewer isolated vertices than $F$, so $\mathbb{E}\left[\operatorname{inj}\left(F^{\prime \prime}(\ell), \mathbb{G}\right)\right]=\mathbb{E}\left[\operatorname{inj}\left(F^{\prime \prime}(\ell), \mathbb{G}^{\prime}\right)\right]$ for each $F^{\prime \prime}(\ell)$, and $\operatorname{inj}^{*}\left(F^{\prime}, k, \mathbb{G}\right)$ and $\operatorname{inj}^{*}\left(F^{\prime}, k, \mathbb{G}^{\prime}\right)$ are each a finite sum of such terms. Therefore, for $\mathbb{G}^{\prime}$,

$$
\sum_{k=1}^{\infty} \frac{\mathbb{E}\left[\operatorname{inj}^{*}\left(F^{\prime}, k, \mathbb{G}^{\prime}\right)\right]}{k !}<\infty
$$


We therefore have

$$
\begin{aligned}
\mathbb{E}[\operatorname{inj}(F, \mathbb{G})] & =\sum_{k=1}^{\infty} \frac{(-1)^{k-1}}{k !} \mathbb{E}\left[\operatorname{inj}^{*}\left(F^{\prime}, k, \mathbb{G}\right)\right] \\
& =\sum_{k=1}^{\infty} \frac{(-1)^{k-1}}{k !} \mathbb{E}\left[\operatorname{inj}^{*}\left(F^{\prime}, k, \mathbb{G}^{\prime}\right)\right]=\mathbb{E}\left[\operatorname{inj}\left(F, \mathbb{G}^{\prime}\right)\right]
\end{aligned}
$$

Lemma 6.12. Suppose we have two random finite graphs, $\mathbb{G}$ and $\mathbb{G}^{\prime}$. Suppose that for every finite graph $F, \mathbb{E}[\operatorname{inj}(F, \mathbb{G})]=\mathbb{E}\left[\operatorname{inj}\left(F, \mathbb{G}^{\prime}\right)\right]$. Let $X$ be the random variable that gives the number of vertices in $\mathbb{G}$, and suppose that for some $\varepsilon>0$, $\mathbb{E}\left[(2+\varepsilon)^{X}\right]$ is finite. Then $\mathbb{G}$ and $\mathbb{G}^{\prime}$ give rise to the same distribution on graphs (up to isomorphism).

We would like to emphasize that in these two lemmas, it suffices to assume the finiteness condition for $\mathbb{G}$, not $\mathbb{G}^{\prime}$ (for which it follows).

Proof. For graphs $F$ and $G$, let $X(F, G)$ be equal to the random variable which is equal to $\operatorname{inj}(F, G)$ if $G$ has the same number of vertices as $F$, and gives 0 otherwise. Fix a graph $F$ with $k$ vertices, and let $F_{i}$ be the graph obtained by adding $i$ isolated vertices to $F$. We can then write

$$
X(F, G)=\sum_{i=0}^{\infty} \frac{(-1)^{i}}{i !} \operatorname{inj}\left(F_{i}, \mathbb{G}\right) .
$$

Indeed, if $G$ has the same number of vertices as $F$, then each term with $i>0$ is zero, and the $i=0$ term gives $\operatorname{inj}\left(F_{0}, G\right)=\operatorname{inj}(F, G)$. If $G$ has fewer vertices, then the entire expression is zero. If $G$ has $k+\ell$ vertices where $\ell>0$, then each injective copy of $G$ contributes (since $\left(\begin{array}{l}\ell \\ i\end{array}\right)=0$ if $i>\ell$ )

$$
\sum_{i=0}^{\ell}(-1)^{i}\left(\begin{array}{l}
\ell \\
i
\end{array}\right)=0
$$

therefore the entire expression is 0 . Next, we claim that

$$
\sum_{i=0}^{\infty} \frac{\mathbb{E}\left[\operatorname{inj}\left(F_{i}, \mathbb{G}\right)\right]}{i !}<\infty
$$

To see this, note that for every $i$,

$$
\mathbb{E}\left[\operatorname{inj}\left(F_{i}, \mathbb{G}\right)\right] \leq \sum_{n} \mathbb{P}(X=n)(n)_{k+i} .
$$

By the condition on $X$, there exists an $\varepsilon>0$ and $c$ such that $\mathbb{P}(X=n) \leq c(2+\varepsilon)^{-n}$. Therefore,

$$
\begin{aligned}
\sum_{i=0}^{\infty} \frac{\mathbb{E}\left[\operatorname{inj}\left(F_{i}, \mathbb{G}\right)\right]}{i !} & \leq \sum_{i} \sum_{n} c(2+\varepsilon)^{-n}(n)_{k}\left(\begin{array}{c}
n-k \\
i
\end{array}\right) \\
& =\sum_{n} c(2+\varepsilon)^{-n} 2^{n-k}(n)_{k}<\infty .
\end{aligned}
$$

We therefore have that

$$
\mathbb{E}[X(F, \mathbb{G})]=\sum_{i=0}^{\infty} \frac{(-1)^{i}}{i !} \mathbb{E}\left[\operatorname{inj}\left(F_{i}, \mathbb{G}\right)\right]=\sum_{i=0}^{\infty} \frac{(-1)^{i}}{i !} \mathbb{E}\left[\operatorname{inj}\left(F_{i}, \mathbb{G}^{\prime}\right)\right]=\mathbb{E}\left[X\left(F, \mathbb{G}^{\prime}\right)\right] .
$$


Thus, by an inclusion-exclusion formula, we can express the probability that $\mathbb{G}$ and $\mathbb{G}^{\prime}$ is isomorphic to a graph $F$ for any $F$, and the two probabilities must also be equal. This completes the proof.

Proof of Theorem 6.10. We first show that (1) implies (2). Recall that for any graph $F, \mathbb{E}\left[\operatorname{inj}\left(F, \mathbb{G}_{n}\right)\right]$ and $\mathbb{E}[\operatorname{inj}(F, \mathbb{G})]$ are uniformly bounded. Fix a graph $F$ on $k$ vertices. We claim that $\mathbb{E}\left[\operatorname{inj}\left(F, \mathbb{G}_{n}\right)^{2}\right]$ and $\mathbb{E}\left[\operatorname{inj}(F, \mathbb{G})^{2}\right]$ are uniformly bounded. This follows from the fact that for a graph $G, \operatorname{inj}(F, G)^{2}$ is a linear combination of the form $\sum_{F^{\prime}} c_{F^{\prime}} \operatorname{inj}\left(F^{\prime}, G\right)$, where $c_{F^{\prime}}$ is independent of $G$ and zero for all but a finite number of graphs $F^{\prime}$. We also know that $\operatorname{inj}\left(F, \mathbb{G}_{n}\right)$ converges to $\operatorname{inj}(F, \mathbb{G})$ in distribution. Since their second moments are uniformly bounded, their expectations must converge as well.

It is clear that (2) implies (3). Let us show that (3) implies (1). Assume $\mathbb{G}_{n}$ satisfies (3). We first claim that the sequence $\mathbb{G}_{n}$ is tight. That is, we claim that for every $\varepsilon$, there exists a finite set of graphs such that for each $n$, with probability at least $1-\varepsilon, \mathbb{G}_{n}$ is in this set. Indeed, the expected number of edges $\mathbb{E}\left[\operatorname{inj}\left(K_{2}, \mathbb{G}_{n}\right)\right]$ is uniformly bounded, which means that for any $\varepsilon$, there exists an $M$ such that the probability of having more than $M$ edges is at most $\varepsilon$. But the number of graphs with $M$ edges and no isolated vertices is finite; therefore $\mathbb{G}_{n}$ is tight.

This means that there is a subsequence that converges to a random graph $\mathbb{H}$ in the sense of (1), which also has no isolated vertices. First, we claim that for any $F$ with no isolated vertices, $\mathbb{E}[\operatorname{inj}(F, \mathbb{H})]$ is finite, and $\mathbb{E}\left[\operatorname{inj}\left(F, \mathbb{G}_{n}\right)\right]$ converges to it. This again follows from the fact that $\mathbb{E}\left[\operatorname{inj}\left(F, \mathbb{G}_{n}\right)^{2}\right]$ is uniformly bounded. But then this implies that $\mathbb{E}[\operatorname{inj}(F, \mathbb{H})]=\mathbb{E}[\operatorname{inj}(F, \mathbb{G})]$ for every graph $F$ with no isolated vertices. Therefore, the two distributions are equal by Lemmas 6.11 and 6.12.

Lemma 6.13. Given $t>0$, let $t^{\prime}=2 t+\log 4$. Then the following holds for any positive integer $n$ and any graph $G$ on $n$ vertices with no isolated vertices. Suppose we randomly color the vertices red and blue, and let $X$ be the number of vertices that are colored red, and have at least one blue neighbor. Then

$$
\mathbb{E}\left[e^{t^{\prime} X}\right] \geq e^{t n}
$$

Proof. Without loss of generality, we may assume $G$ is the disjoint union of stars, since otherwise we can delete an edge and $G$ will still have no isolated vertices. Suppose that $G$ is the disjoint union of stars with edge count $s_{1}, s_{2}, \ldots, s_{k}$, where each $s_{i} \geq 1$ and $\sum_{i} s_{i}=n-k$. Note that $k \leq n / 2$. Then

$$
\begin{aligned}
\mathbb{E}\left[e^{t^{\prime} X}\right] & =\prod_{i=1}^{k}\left(\frac{1}{2}\left(\frac{e^{t^{\prime}}+1}{2}\right)^{s_{k}}+\frac{1}{2}\left(\frac{e^{t^{\prime}}+2^{s_{k}}-1}{2^{s_{k}}}\right)\right) \\
& \geq\left(\frac{e^{t^{\prime}}+1}{2}\right)^{n-k} 2^{-k} \geq\left(e^{t^{\prime}}+1\right)^{n / 2} 2^{-n} \geq e^{t n},
\end{aligned}
$$

given that our choice of $t^{\prime}$ implies that $\left(e^{t^{\prime}}+1\right) / 4 \geq e^{2 t}$.

Lemma 6.14. For any $T, t, C, D \in \mathbb{R}_{+}$, there exists a finite $B$ such that the following holds. Suppose we have a graphex $\mathbb{W}$, with $\left\|D_{\mathbb{W}}\right\|_{\infty} \leq D$ and $\|\mathbb{W}\|_{1} \leq C$. Let $X$ be the number of vertices (that are not isolated) of $G_{T}(\mathbb{W})$. Then $\mathbb{E}\left[e^{t X}\right] \leq B$.

Proof. Note that since $X$ is nonnegative, we only need to worry about $t>0$. First, let $X^{\prime}$ be obtained by randomly coloring the vertices of $G_{T}$ red and blue, and 
taking the red vertices with at least one blue neighbor. By the above lemma, for $t^{\prime}=2 t+\log 4>0$, we have

$$
\mathbb{E}\left[e^{t^{\prime} X^{\prime}}\right] \geq \mathbb{E}\left[e^{t X}\right] .
$$

We claim that

$$
\mathbb{E}\left[e^{t^{\prime} X^{\prime}}\right] \leq e^{\frac{T^{2} I}{2}\left(e^{t^{\prime}}-1\right)} \exp \left(\frac{T}{2} \int_{\Omega}\left(e^{\frac{T}{2} D_{\mathbb{W}}(x)\left(e^{t^{\prime}}-1\right)}-1\right) d \mu(x)\right) .
$$

Let us show how this implies uniform boundedness. We know that $D_{\mathbb{W}}$ is bounded by $D$. Since the function $z \rightarrow e^{z}-1$ is 0 at $z=0$ and convex, there exists a constant $K$ depending only on $t^{\prime}, T$, and $D$ such that

$$
e^{\frac{T}{2} D_{\mathbb{W}}(x)\left(e^{t^{\prime}}-1\right)}-1 \leq \frac{D_{\mathbb{W}}(x)}{D} e^{\frac{T}{2} D\left(e^{t^{\prime}}-1\right)}=K D_{\mathbb{W}}(x) .
$$

Therefore,

$$
\begin{aligned}
\frac{T^{2} I}{2}\left(e^{t^{\prime}}-1\right)+ & \frac{T}{2} \int_{\Omega}\left(e^{\frac{T}{2} D_{\mathbb{W}}(x)\left(e^{t^{\prime}}-1\right)}-1\right) d \mu(x) \\
& \leq \frac{T^{2} I}{2}\left(e^{t^{\prime}}-1\right)+\frac{T}{2} \int_{\Omega} K D_{\mathbb{W}}(x) d \mu(x) \\
& \leq \frac{T^{2} C}{4}\left(e^{t^{\prime}}-1\right)+\frac{K T C}{2} .
\end{aligned}
$$

In order to prove (6.1), we first show that it is enough to consider the case where $\mu(\Omega)<\infty$. To see this, we write a general $\sigma$-finite measure space as the union of finite spaces, $\Omega=\bigcup_{n} \Omega_{n}$ where $\Omega_{n}$ is an increasing sequence with $\mu\left(\Omega_{n}\right)<\infty$ for all $n$. For a fixed $T$, let $G_{T, n}$ consist of the induced graph on those vertices which were born in $\Omega_{n}$, come from a star of a vertex born in $\Omega_{n}$, or come from a dust edge, and define $X_{n}^{\prime}$ to be the number of red vertices in $G_{T, n}$ with at least one blue neighbor in $G_{T, n}$. It is easy to see that $0 \leq X_{1}^{\prime} \leq X_{2}^{\prime} \leq \cdots \leq X_{n}^{\prime} \leq \ldots$ By monotone convergence, the expectation of $e^{t^{\prime} X_{n}^{\prime}}$ converges to $\mathbb{E}\left[e^{t^{\prime} X^{\prime}}\right]$. The bound we obtain also converges, giving the required bound on $\mathbb{E}\left[e^{t^{\prime} X^{\prime}}\right]$.

Assume thus that $\mu(\Omega)<\infty$. In this case, almost surely, a finite number of blue points will be created. Suppose that these are $x_{1}, x_{2}, \ldots, x_{k}$. Conditioned on this, red vertices that have blue neighbors can be created as follows. They can be created by the Poisson process on $\Omega$, and then be connected to at least one of the $x_{i}$. If a point is created at $x$, the probability that it is connected to at least one of the $x_{i}$ is $1-\prod\left(1-W\left(x_{i}, x\right)\right)$. It can also be created as a leaf of a star created at one of the $x_{i}$. Finally, it can be created by a dust edge being colored red and blue. Since the number of red vertices coming from each of these cases is independent, the number of red vertices with at least one blue neighbor is a Poisson distribution with expectation

$$
\begin{aligned}
f\left(x_{1}, \ldots, x_{k}\right) & :=\frac{T}{2} \int_{\Omega}\left(1-\prod_{i=1}^{k}\left(1-W\left(x_{i}, x\right)\right)\right) d \mu(x)+\frac{T}{2} \sum_{i=1}^{k} S\left(x_{i}\right)+\frac{T^{2} I}{2} \\
& \leq \frac{T}{2} \int_{\Omega}\left(\sum_{i=1}^{k} W\left(x_{i}, x\right)\right) d \mu(x)+\frac{T}{2} \sum_{i=1}^{k} S\left(x_{i}\right)+\frac{T^{2} I}{2} \\
& =\frac{T}{2} \sum_{i=1}^{k} D_{\mathbb{W}}\left(x_{i}\right)+\frac{T^{2} I}{2} .
\end{aligned}
$$


In particular, this means that (for $t^{\prime}>0$ ) we have

$$
\mathbb{E}\left[e^{t^{\prime} X^{\prime}} \mid x_{1}, x_{2}, \ldots, x_{k}\right]=e^{f\left(x_{1}, \ldots, x_{k}\right)\left(e^{t^{\prime}}-1\right)} \leq e^{\frac{T}{2}\left(T I+\sum_{i=1}^{k} D_{\mathbb{W}}\left(x_{i}\right)\right)\left(e^{t^{\prime}}-1\right)} .
$$

Therefore

$$
\begin{aligned}
\mathbb{E}\left[e^{t^{\prime} X^{\prime}}\right] & =\sum_{k=0}^{\infty} e^{-\frac{T}{2} \mu(\Omega)} \frac{(T / 2)^{k} \mu(\Omega)^{k}}{k !} \frac{1}{\mu(\Omega)^{k}} \int_{\Omega^{k}} e^{f\left(x_{1}, x_{2}, \ldots, x_{k}\right)\left(e^{t^{\prime}}-1\right)} d \mu\left(x_{1}\right) \ldots d \mu\left(x_{k}\right) \\
& \leq \sum_{k=0}^{\infty} e^{-\frac{T}{2} \mu(\Omega)} \frac{(T / 2)^{k}}{k !} \int_{\Omega^{k}} e^{\frac{T}{2}\left(T I+\sum_{i=1}^{k} D_{\mathbb{W}}\left(x_{i}\right)\right)\left(e^{t^{\prime}}-1\right)} d \mu\left(x_{1}\right) \ldots d \mu\left(x_{k}\right) \\
& =e^{\frac{T^{2} I}{2}\left(e^{t^{\prime}}-1\right)} \sum_{k=0}^{\infty} e^{-\frac{T}{2} \mu(\Omega)} \frac{(T / 2)^{k}}{k !}\left(\int_{\Omega} e^{\frac{T}{2} D_{\mathbb{W}}(x)\left(e^{t^{\prime}}-1\right)} d \mu(x)\right)^{k} .
\end{aligned}
$$

Here we think of $\Omega^{0}$ as consisting of a single point on which $f$ is 0 . We then have

$$
\begin{aligned}
\mathbb{E}\left[e^{t^{\prime} X^{\prime}}\right] & \leq e^{\frac{T^{2} I}{2}\left(e^{t^{\prime}}-1\right)} e^{\frac{T}{2} \int_{\Omega} e^{\frac{T}{2} D_{\mathrm{W}}(x)\left(e^{t^{\prime}}-1\right)} d \mu(x)-\frac{T}{2} \mu(\Omega)} \\
& =e^{\frac{T^{2} I}{2}\left(e^{t^{\prime}}-1\right)} e^{\frac{T}{2} \int_{\Omega}\left(e^{\frac{T}{2} D_{\mathrm{W}}(x)\left(e^{t^{\prime}}-1\right)}-1\right) d \mu(x)} .
\end{aligned}
$$

So we know that (6.1) is true for any $\Omega$ with finite measure. This completes the proof of the lemma.

After these preparations, the proof of Theorem 6.3 is straightforward.

Proof of Theorem 6.3. We first note that it is enough to prove the lemma for the case that $\mathbb{W}_{n}$ and $\mathbb{W}$ are $(C, D)$-bounded for some finite $C, D<\infty$. Indeed, both (3) and (4) clearly imply a bound on the $\|\cdot\|_{1}$-norm, but also (2) (and therefore (1)) does, since (2) implies that the random graphs $G_{T}\left(\mathbb{W}_{n}\right)$ are tight, which implies that the set of graphexes is tight, which by Corollary 4.2 (2) implies uniform boundedness of the $\|\cdot\|_{1}$-norms.

Assume thus that $\mathbb{W}_{n}$ and $\mathbb{W}$ are $(C, D)$-bounded for some finite $C, D<\infty$. The equivalence of (3) and (4) follows from the fact that $t$ is multiplicative over components of $F$. (1) $\Rightarrow(2)$ is obvious. Using Lemma 6.14 , we can apply the equivalence in Theorem 6.10 and Proposition 3.24 to show that $(2) \Rightarrow(3)$ and $(3) \Rightarrow(1)$.

A slight modification of the above proof gives the following theorem.

Theorem 6.15. Given two graphexes $\mathbb{W}, \mathbb{W}^{\prime}$ with bounded marginals, the following are equivalent:

(1) $G_{T}(\mathbb{W})$ and $G_{T}\left(\mathbb{W}^{\prime}\right)$ have the same distribution for every $T$.

(2) $G_{T}(\mathbb{W})$ and $G_{T}\left(\mathbb{W}^{\prime}\right)$ have the same distribution for some $T$.

(3) For every graph $F$ with no isolated vertices, $t(F, \mathbb{W})=t\left(F, \mathbb{W}^{\prime}\right)$.

(4) For every connected graph $F, t(F, \mathbb{W})=t\left(F, \mathbb{W}^{\prime}\right)$.

Proof. As before, the equivalence of (4) and (3) follows from the product property of $t$. The implication (1) $\Rightarrow(2)$ is obvious. To prove $(2) \Rightarrow(3)$, we use the fact that $t(F, \mathbb{W})=T^{-|V(F)|} \mathbb{E}\left[\operatorname{inj}\left(F, G_{T}(\mathbb{W})\right)\right]$ and the same holds for $\mathbb{W}^{\prime}$. Since $\operatorname{inj}\left(F, G_{T}(\mathbb{W})\right)$ and $\operatorname{inj}\left(F, G_{T}\left(\mathbb{W}^{\prime}\right)\right)$ have the same distribution, their expectations must be equal. With the help of Proposition 3.24, this implies (3). (3) $\Rightarrow(1)$ follows from Proposition 3.24, the observation that graphexes with bounded marginals are integrable, and Lemmas 6.14, 6.11, and 6.12. 
6.3. Metric convergence implies GP-convergence. We close this section by proving that under the assumption of uniformly bounded marginals, $\delta_{2 \rightarrow 2}$-convergence implies GP-convergence. We then use this result to show that without any assumptions on the marginals, $\delta_{\diamond}$-convergence implies GP-convergence.

Theorem 6.16. Suppose $\mathbb{W}_{n}$ and $\mathbb{W}$ have uniformly bounded marginals, and $\delta_{2 \rightarrow 2}\left(\mathbb{W}_{n}, \mathbb{W}\right) \rightarrow 0$. Then $\mathbb{W}_{n}$ is GP-convergent to $\mathbb{W}$.

Proof. Note that $\delta_{2 \rightarrow 2}$ convergence implies that $\left\|\mathbb{W}_{n}\right\|_{1} \rightarrow\|\mathbb{W}\| ;$ therefore the sequence is $(C, D)$-bounded for some $C, D$. If $F$ is a graph without isolated vertices, then $t\left(F, \mathbb{W}_{n}\right) \rightarrow t(F, \mathbb{W})$ by Corollary 6.2. Therefore, by Theorem 6.3, for any $T$, $G_{T}\left(\mathbb{W}_{n}\right)$ converges to $G_{T}(\mathbb{W})$ in distribution.

Theorem 6.17. Suppose that graphexes $\mathbb{W}$ and $\left(\mathbb{W}_{n}\right)_{n=1}^{\infty}$ have the property that $\delta_{\diamond}\left(\mathbb{W}_{n}, \mathbb{W}\right) \rightarrow 0$. Then $\mathbb{W}_{n}$ is GP-convergent to $\mathbb{W}$.

Proof. By Proposition 4.6, the sequence is tight, and for all $D$ such that $\mu\left(\left\{D_{\mathbb{W}}=\right.\right.$ $D\})=0$, we have that $\mu_{n}\left(\Omega_{n,>D}\right) \rightarrow \mu\left(\Omega_{>D}\right)$ and $\delta_{2 \rightarrow 2}\left(\mathbb{W}_{n, \leq D}, \mathbb{W}_{\leq D}\right) \rightarrow 0$.

Fix $T$ and $\varepsilon>0$, and take $\delta$ small enough so that for all sets $\Omega_{\delta}$ of measure at most $\delta$ the probability that any of the vertices in $G_{T}$ has a feature in $\Omega_{\delta}$ is at most $\varepsilon / 3$. Take $D$ large enough so that for all $n, \mu_{n}\left(\Omega_{n,>D}\right)$ and $\mu\left(\Omega_{>D}\right)$ are at most $\delta$. Then the total variation distance between $G_{T}\left(\mathbb{W}_{n, \leq D}\right)$ and $G_{T}\left(\mathbb{W}_{n}\right)$ is at most $\varepsilon / 3$, and the same is true for $G_{T}\left(\mathbb{W}_{\leq D}\right)$ and $G_{T}(\mathbb{W})$. We also know that $\delta_{2 \rightarrow 2}\left(\mathbb{W}_{n, \leq D}, \mathbb{W}_{\leq D}\right) \rightarrow 0$, which in particular implies that the sequence is uniformly $(C, D)$-bounded for some $C$. Therefore, it is GP-convergent. In particular, for $n$ large enough, the total variation distance between $G_{T}\left(\mathbb{W}_{n, \leq D}\right)$ and $G_{T}\left(\mathbb{W}_{\leq D}\right)$ is at most $\varepsilon / 3$. This implies that for $n$ large enough, the total variation distance between $G_{T}\left(\mathbb{W}_{n}\right)$ and $G_{T}(\mathbb{W})$ is at most $\varepsilon$, which shows that the sequence is GP-convergent.

\section{SAMPLing}

In this section, we prove that GP-convergence implies convergence in the weak kernel metric, completing the proof of the equivalence of convergence in the metric $\delta_{\diamond}$ and GP-convergence (Theorem 2.18). The main technical tool to establish this will be a "sampling lemma", showing that as $T \rightarrow \infty$, the graphs $G_{T}(\mathbb{W})$ sampled from a graphex $\mathbb{W}$ converge to the generating graphex according to $\delta_{\diamond}$.

To make this precise, we need a way to compare graphs to graphexes. As in [3] and [4], we do this by transforming the graph into a suitable "empirical graphon" and corresponding "empirical graphex". Differing slightly from both [3] and [4], where the empirical graphon was a graphon over $\mathbb{R}_{+}$, here we define it to be a graphon over the vertex set of the graph. Explicitly, given a finite graph $G$ and $\rho>0$, we define the graphon $W(G, \rho)$ as follows. Let $\Omega=(\Omega, \mathcal{F}, \mu)$, where $\Omega$ is the set of vertices, $\mathcal{F}$ is the $\sigma$-algebra consisting of all subsets, and $\mu$ is the measure where each vertex has weight $\rho$. Set $W(x, y)$ to be 1 if there is an edge between the corresponding vertices, and 0 otherwise. This gives us the graphon $W(G, \rho)$. We then set $\mathbb{W}(G, \rho)=(W, 0,0, \boldsymbol{\Omega})$. Similarly, if $H$ is a weighted graph with countably many vertices, we define $\Omega$ to be the set of vertices, $\mathcal{F}$ to be the $\sigma$-algebra consisting of all subsets of $\Omega$, and $\mu$ to be the $\sigma$-finite measure which gives weight $\rho$ to each vertex; $W(H, \rho)$ and $\mathbb{W}(H, \rho)$ are then the graphon and graphex obtained by taking $W$ according to edge weights.

With these definitions, we are ready to state the sampling lemma. 
Theorem 7.1. For every graphex $\mathbb{W}$ and $\varepsilon>0$,

$$
\lim _{T \rightarrow \infty} \mathbb{P}\left[\delta_{\diamond}\left(\mathbb{W}\left(G_{T}(\mathbb{W}), 1 / T\right), \mathbb{W}\right)>\varepsilon\right]=0 .
$$

For a set of graphexes that is tight, the convergence is uniform.

Remark 7.2. The above theorem only claims convergence in probability. However, once we establish equivalence of GP-convergence and convergence in the weak kernel norm, the results of [18] imply convergence with probability one (since there convergence with probability one is proved for GP-convergence). Nevertheless, to establish the equivalence, all we need is convergence in probability, so this is all we will prove here.

7.1. Closeness of graphexes implies closeness of samples. In order to prove the sampling lemma, we will first prove that two graphexes with bounded marginals that are close in the kernel metric lead to samples that are close. This is formalized in the following theorem.

Theorem 7.3. Suppose $\mathbb{W}_{1}, \mathbb{W}_{2}$ are two $(C, D)$-bounded graphexes on the same space $\boldsymbol{\Omega}$, and suppose that $d_{2 \rightarrow 2}\left(\mathbb{W}_{1}, \mathbb{W}_{2}\right) \leq c$ for some $0<c<1$. Then there exists a $T_{0}$ (depending only on $c, C$, and $D$ ) such that for any $T>T_{0}$, there exists a coupling of the random graphs $G_{T}\left(\mathbb{W}_{1}\right)$ and $G_{T}\left(\mathbb{W}_{2}\right)$ so that

$\mathbb{P}\left[\delta_{2 \rightarrow 2}\left(\mathbb{W}\left(G_{T}\left(\mathbb{W}_{1}\right), 1 / T\right), \mathbb{W}\left(G_{T}\left(\mathbb{W}_{2}\right), 1 / T\right)\right)>\min \left((31 c C)^{1 / 4}, 2 c^{3 / 4}, \sqrt[3]{3} c\right)\right]<c$.

For graphons, or graphexes with only a graphon part, we can think of obtaining $G_{T}$ as having two phases: first we sample the set of vertices, and then we sample the edges according to the edge probability. If we do not do the second phase, we obtain a weighted graph. We will work with this intermediate graph in this section. To make this precise, given a graphon $(W, \boldsymbol{\Omega})$, define $H_{T}(W)$ as the random weighted graph where we take a Poisson process on $\boldsymbol{\Omega} \times[0, T]$, set these to be the vertices of $H_{T}(W)$, and for each pair of vertices $\left(x_{i}, t_{i}\right)$ and $\left(x_{j}, t_{j}\right)$, put a weighted edge with weight $W\left(x_{i}, x_{j}\right)$ (with 0 weights on the diagonals).

In order to prove the theorem, we need to find a coupling of the random processes that provide $G_{T}\left(\mathbb{W}_{1}\right)$ and $G_{T}\left(\mathbb{W}_{2}\right)$. Since $\mathbb{W}_{1}$ and $\mathbb{W}_{2}$ have the same underlying space, it is natural to couple the Poisson processes that generate the vertices into a single Poisson process. Conditioned on this, we generate the two random graphs independently (this is not optimal but it is satisfactory for our purposes). Let $\mathbb{W}_{i}^{\prime}=\mathbb{W}_{i}\left(G_{T}\left(\mathbb{W}_{i}\right), 1 / T\right)=\left(W_{i}^{\prime}, S_{i}^{\prime}, I_{i}^{\prime}, \boldsymbol{\Omega}_{i}^{\prime}\right)$. The underlying space of $\mathbb{W}_{i}^{\prime}$ consists of the vertex set of $G_{T}\left(\mathbb{W}_{i}\right)$, everything with weight $1 / T$. We couple the two underlying spaces by matching vertices that correspond to the same point in $\Omega$, and couple the other vertices arbitrarily (adding points with degree 0 if necessary). We will show that in this way, all three components of our distance will be close

Let us first show that $\left\|W_{1}^{\prime}-W_{2}^{\prime}\right\|_{2 \rightarrow 2}$ is small, with high probability. Note that $G_{T}(\mathbb{W})$ consists of the edges in $G_{T}(W)$, and the edges generated by the stars and the independent edges. In the following lemma, we show that the extra edges generated have a small effect on this distance

Lemma 7.4. Let $\mathbb{W}=(W, S, I, \boldsymbol{\Omega})$ be a $(C, D)$-bounded graphex, and $T>1 / D$. Let $G_{T}(\mathbb{W})$ be the usual sample at time $T$, and let $\widetilde{G}_{T}(\mathbb{W})$ consist of only those 
edges which come from $I$ or $S$. Then

$$
\mathbb{P}\left[\left\|W\left(\widetilde{G}_{T}(\mathbb{W}), 1 / T\right)\right\|_{2 \rightarrow 2}>\left(\frac{2 C D}{\sqrt{T}}\right)^{1 / 4}\right]<\frac{1}{\sqrt{T}} .
$$

Proof. Suppose we have sampled stars with $s_{1}, s_{2}, \ldots, s_{\ell}$ leaves, and we have sampled $m$ isolated edges. Let $U=W\left(\widetilde{G}_{T}(\mathbb{W}), 1 / T\right)$. Then

$$
t\left(C_{4}, U\right)=\frac{1}{T^{4}}\left(2 \sum_{i} s_{i}^{2}+2 m\right) .
$$

Therefore,

$$
\mathbb{E}\left[t\left(C_{4}, U\right)\right]=\frac{2 T \int_{\Omega}\left(T^{2} S(x)^{2}+T S(x)\right) d \mu(x)}{T^{4}}+\frac{2 T^{2} I}{T^{4}} \leq \frac{C(D+1 / T)}{T} \leq \frac{2 C D}{T}
$$

and hence

$$
\mathbb{P}\left[t\left(C_{4}, U\right)>\frac{2 C D}{\sqrt{T}}\right]<\frac{1}{\sqrt{T}} .
$$

Using the fact that $\|U\|_{2 \rightarrow 2} \leq t\left(C_{4}, U\right)^{1 / 4}$ (Lemma 3.18), the lemma follows.

This lemma implies that for the $2 \rightarrow 2$ component of the distance, we can compare $G_{T}\left(W_{1}\right)$ and $G_{T}\left(W_{2}\right)$ instead of $G_{T}\left(\mathbb{W}_{1}\right)$ and $G_{T}\left(\mathbb{W}_{2}\right)$. The following lemma will imply that it in fact suffices to compare $H_{T}\left(W_{1}\right)$ and $H_{T}\left(W_{2}\right)$, because $G_{T}$ is close to $H_{T}$, as long as $H_{T}\left(W_{i}\right)$ satisfies certain boundedness conditions (which, by the boundedness of the $W_{i}$, will be satisfied with high probability).

Lemma 7.5. Suppose $H$ is a weighted graph on $\mathbb{N}$ with weights $H_{i, j} \in[0,1]$, and $H_{i, i}=0$. Suppose that $G$ is generated by taking an edge between $i$ and $j$ with probability $H_{i, j}$, independently for every pair of vertices. Suppose that $\sum_{i, j} H_{i, j} \leq E$ and $\sum_{i, j, k} H_{i, j} H_{j, k} \leq F$ where the sum goes over pairwise distinct vertices. Let $0<\rho$. Then

$$
\mathbb{P}\left[\|\mathbb{W}(G, \rho)-\mathbb{W}(H, \rho)\|_{2 \rightarrow 2}>\rho^{7 / 8}(E+2 F)^{1 / 4}\right]<\sqrt{\rho} .
$$

Proof. Let $X_{i, j}=G_{i, j}-H_{i, j}$. Notice that $\mathbb{E} X_{i, j}=0$ and $X_{i, j}$ over different pairs are independent. Also, each $X_{i, i}=0$. Therefore,

$$
\begin{aligned}
\mathbb{E}\left[t\left(C_{4}, \mathbb{W}(G, \rho)-\mathbb{W}(H, \rho)\right)\right]=\rho^{4} \mathbb{E}\left[\sum_{i, j} X_{i, j}^{4}+2 \sum_{i, j, k} X_{i, j}^{2} X_{j, k}^{2}\right. \\
\left.+\sum_{i, j, k, l} X_{i, j} X_{j, k} X_{k, l} X_{l, i}\right],
\end{aligned}
$$

where in each of the sums, all indices are pairwise distinct. Here

$$
\sum_{i, j} \mathbb{E}\left[X_{i, j}^{4}\right]=\sum_{i, j}\left(H_{i, j}\left(1-H_{i, j}\right)^{4}+\left(1-H_{i, j}\right) H_{i, j}^{4}\right) \leq \sum_{i, j} H_{i, j} \leq E .
$$

Also,

$$
\begin{aligned}
\sum_{i, j, k} \mathbb{E}\left[X_{i, j}^{2} X_{j, k}^{2}\right] & =\sum_{i, j, k}\left(H_{i, j}-2 H_{i, j}^{2}+H_{i, j}^{2}\right)\left(H_{j, k}-2 H_{j, k}^{2}+H_{j, k}^{2}\right) \\
& =\sum_{i, j, k} H_{i, j}\left(1-H_{i, j}\right) H_{j, k}\left(1-H_{j, k}\right) \leq \sum_{i, j, k} H_{i, j} H_{j, k} \\
& \leq F
\end{aligned}
$$


Finally, for any pairwise distinct $i, j, k, \ell$,

$$
\mathbb{E}\left[X_{i, j} X_{j, k} X_{k, \ell} X_{\ell, i}\right]=0 .
$$

Therefore,

$$
0 \leq \mathbb{E}\left[t\left(C_{4}, \mathbb{W}(G, \rho)-\mathbb{W}(H, \rho)\right)\right] \leq \rho^{4}(E+2 F) .
$$

This implies that

$$
\mathbb{P}\left[t\left(C_{4}, \mathbb{W}(G, \rho)-\mathbb{W}(H, \rho)\right)>\rho^{7 / 2}(E+2 F)\right]<\sqrt{\rho}
$$

Using the fact that $\|U\|_{2 \rightarrow 2} \leq\left(t\left(C_{4}, U\right)\right)^{1 / 4}$ (Lemma 3.18), the lemma follows.

Proof of Theorem 7.3. We are now ready to show that with high probability, $\| W_{1}^{\prime}-$ $W_{2}^{\prime} \|_{2 \rightarrow 2}$ is small. Recall that we have a coupling of $H_{T}\left(W_{1}\right)$ and $H_{T}\left(W_{2}\right)$ such that $H_{T}\left(W_{1}\right)-H_{T}\left(W_{2}\right)=H_{T}\left(W_{1}-W_{2}\right)$ with probability one. Let $U=W_{1}-W_{2}$, so that $H_{T}\left(W_{1}\right)-H_{T}\left(W_{2}\right)=H_{T}(U)$.

Let us first show that $\left\|W\left(H_{T}(U), 1 / T\right)\right\|_{2 \rightarrow 2}$ is small. First, suppose that $\mu=$ $\mu(\Omega)$ is finite. Let

$$
\begin{gathered}
X=\int_{\Omega^{2}} U\left(x_{1}, x_{2}\right)^{4} d \mu\left(x_{1}\right) d \mu\left(x_{2}\right) \leq 2 C, \\
Y=\int_{\Omega^{3}} U\left(x_{1}, x_{2}\right)^{2} U\left(x_{2}, x_{3}\right)^{2} d \mu\left(x_{1}\right) d \mu\left(x_{2}\right) d \mu\left(x_{3}\right) \leq 4 C D,
\end{gathered}
$$

and

$$
\begin{aligned}
Z & =\int_{\Omega^{4}} U\left(x_{1}, x_{2}\right) U\left(x_{2}, x_{3}\right) U\left(x_{3}, x_{4}\right) U\left(x_{4}, x_{1}\right)=t\left(C_{4}, U\right) \\
& \leq\left\|W_{1}-W_{2}\right\|_{2 \rightarrow 2}^{2}\left\|W_{1}-W_{2}\right\|_{2}^{2} \\
& \leq\left\|W_{1}-W_{2}\right\|_{2 \rightarrow 2}^{2}\left\|W_{1}-W_{2}\right\|_{1} \\
& \leq 2 c^{2} C
\end{aligned}
$$

where we used Lemma 3.18, the fact that both graphexes are $(C, D)$-bounded, and the fact that $d_{2 \rightarrow 2}\left(\mathbb{W}_{1}, \mathbb{W}_{2}\right) \leq c$. If $T>\max \left\{16 \frac{D}{c^{2}}, 2 / c\right\}$, then

$$
\begin{aligned}
\mathbb{E}\left[t\left(C_{4}, H_{T}(U)\right)\right]= & \sum_{n=0}^{\infty} e^{-T \mu} \frac{(T \mu)^{n}}{n !}\left(\frac{n(n-1)}{T^{4} \mu^{2}} X+2 \frac{n(n-1)(n-2)}{T^{4} \mu^{3}} Y\right. \\
& \left.+\frac{n(n-1)(n-2)(n-3)}{T^{4} \mu^{4}} Z\right) \\
\leq & \frac{2 C}{T^{2}}+\frac{8 C D}{T}+2 c^{2} C \leq 3 c^{2} C .
\end{aligned}
$$

In general, we can take a sequence of finite measure subsets $\Omega_{1} \subseteq \cdots \subseteq \Omega_{n} \subseteq \ldots$ with $\bigcup_{n} \Omega_{n}=\Omega$ to show that the above bound on the expectation holds for general $\Omega$. Therefore,

$$
\mathbb{P}\left[\left\|H_{T}(U)\right\|_{2 \rightarrow 2}>(30 c C)^{1 / 4}\right] \leq \mathbb{P}\left[t\left(C_{4}, H_{T}(U)\right)>30 c C\right]<\frac{c}{10} .
$$

Next, let $P_{2}$ be the star with two leaves. If $T$ is large enough, then

$$
\mathbb{E}\left[t\left(P_{2}, H_{T}\left(W_{1}\right)\right)\right]=\left(t\left(P_{2}, W_{1}\right) T^{3}+T^{2}\left\|W_{1}\right\|_{1}\right) \leq C D T^{3}+C T^{2} \leq 2 C D T^{3} .
$$


Therefore,

$$
\mathbb{P}\left[t\left(P_{2}, H_{T}\left(W_{1}\right)\right)>\frac{20 C D T^{3}}{c}\right] \leq \frac{c}{10}
$$

Also, since

$$
\mathbb{E}\left[\left\|H_{T}\left(W_{1}\right)\right\|_{1}\right] \leq C T^{2},
$$

we also have

$$
\mathbb{P}\left[\left\|H_{T}\left(W_{1}\right)\right\|_{1}>\frac{10 C T^{2}}{c}\right] \leq \frac{c}{10}
$$

Conditioned on neither of these happening, we can apply Lemma 7.5 with

$$
E+2 F \leq \frac{10 C T^{2}}{c}+2 \frac{20 C D T^{3}}{c} \leq \frac{50 C D T^{3}}{c}
$$

This means that

$$
\mathbb{P}\left[\left\|\mathbb{W}\left(G_{T}\left(W_{1}\right), 1 / T\right)-\mathbb{W}\left(H_{T}\left(W_{1}\right), 1 / T\right)\right\|_{2 \rightarrow 2}>\left(\frac{50 C D}{c \sqrt{T}}\right)^{1 / 4}\right] \leq \frac{1}{\sqrt{T}} .
$$

Clearly the analogous statements hold for $H_{T}\left(W_{2}\right)$. Let $\widetilde{W}_{i}=W\left(\widetilde{G}_{T}\left(\mathbb{W}_{i}\right), 1 / T\right)$ (i.e., the part consisting of edges generated by the stars and independent edges). Also, let $\widehat{W}_{i}=W\left(G_{T}\left(W_{i}\right), 1 / T\right)-W\left(H_{T}\left(W_{i}\right), 1 / T\right)$. Assuming none of the bad events happen, if $T$ is large enough, then

$$
\begin{gathered}
\left\|W_{1}^{\prime}-W_{2}^{\prime}\right\|_{2 \rightarrow 2} \leq\left\|\widetilde{W}_{1}\right\|_{2 \rightarrow 2}+\left\|\widetilde{W}_{2}\right\|_{2 \rightarrow 2} \\
+\left\|\widehat{W}_{1}\right\|_{2 \rightarrow 2}+\left\|\widehat{W}_{2}\right\|_{2 \rightarrow 2}+\left\|W\left(H_{T}(U), 1 / T\right)\right\|_{2 \rightarrow 2} \\
\leq 2\left(\frac{2 C D}{\sqrt{T}}\right)^{1 / 4}+2\left(\frac{(50 C D)^{1 / 4}}{c \sqrt{T}}\right)^{1 / 4}+(30 c C)^{1 / 4} \leq(31 c C)^{1 / 4} .
\end{gathered}
$$

The probability of one of the bad events happening is at most

$$
\frac{2}{\sqrt{T}}+4 \frac{c}{10}+\frac{2}{\sqrt{T}}+\frac{c}{10} \leq \frac{6 c}{10}
$$

Let us now bound the probability that $\left\|D_{\mathbb{W}_{1}^{\prime}}-D_{\mathbb{W}_{2}^{\prime}}\right\|_{2}$ is large. For $x \in \Omega$, let

$$
D_{\mathbb{W}_{1} \mathbb{W}_{2}}(x)=\int_{\Omega} W_{1}(x, y) W_{2}(x, y) d \mu(y) .
$$

With our coupling,

$$
\begin{aligned}
\mathbb{E}\left[\sum_{v \in V_{T}} d_{G_{T}\left(\mathbb{W}_{1}\right)}(v)^{2}\right] & =\int_{\Omega} T\left(\left(T D_{\mathbb{W}_{1}}(x)\right)^{2}+T D_{\mathbb{W}_{1}}(x)\right) d \mu(x), \\
\mathbb{E}\left[\sum_{v \in V_{T}} d_{G_{T}\left(\mathbb{W}_{1}\right)}(v) d_{G_{T}\left(\mathbb{W}_{2}\right)}(v)\right] & =\int_{\Omega} T\left(\left(T D_{\mathbb{W}_{1}}(x)\right)\left(T D_{\mathbb{W}_{2}}(x)\right)+T D_{\mathbb{W}_{1} \mathbb{W}_{2}}(x)\right) d \mu(x), \\
\mathbb{E}\left[\sum_{v \in V_{T}} d_{G_{T}\left(\mathbb{W}_{2}\right)}(v)^{2}\right] & =\int_{\Omega} T\left(\left(T D_{\mathbb{W}_{2}}(x)\right)^{2}+T D_{\mathbb{W}_{2}}(x)\right) d \mu(x) .
\end{aligned}
$$


Therefore,

$$
\begin{aligned}
\mathbb{E}\left[\sum_{v \in V_{T}}\right. & \left.\left(d_{G_{T}\left(\mathbb{W}_{1}\right)}(x)-d_{G_{T}\left(\mathbb{W}_{2}\right)}(x)\right)^{2}\right] \\
= & T^{3} \int_{\Omega}\left(D_{\mathbb{W}_{1}}(x)-D_{\mathbb{W}_{2}}(x)\right)^{2} d \mu(x) \\
& \quad+T^{2} \int_{\Omega}\left(D_{\mathbb{W}_{1}}(x)+D_{\mathbb{W}_{2}}(x)-2 D_{\mathbb{W}_{1} \mathbb{W}_{2}}(x)\right) d \mu(x) \\
\leq & T^{3}\left\|D_{\mathbb{W}_{1}}-D_{\mathbb{W}_{2}}\right\|_{2}^{2}+T^{2}\left\|\mathbb{W}_{1}\right\|_{1}+T^{2}\left\|\mathbb{W}_{2}\right\|_{1},
\end{aligned}
$$

This means that if $T$ is large enough,

$$
\mathbb{E}\left[\left\|D_{\mathbb{W}_{1}^{\prime}}-D_{\mathbb{W}_{2}^{\prime}}\right\|_{2}^{2}\right] \leq c^{4}+2 C / T \leq 2 c^{4} .
$$

Therefore,

$$
\mathbb{P}\left[\left\|D_{\mathbb{W}_{1}^{\prime}}-D_{\mathbb{W}_{2}^{\prime}}\right\|_{2}>4 c^{3 / 2}\right] \leq \frac{c}{8} .
$$

Finally, recall that by Lemma 4.3 , the number of edges of $G_{T}\left(\mathbb{W}_{i}\right)$ has expectation $T^{2}\left\|\mathbb{W}_{i}\right\|_{1} / 2$ and variance $T^{2}\left\|\mathbb{W}_{i}\right\|_{1} / 2+T^{3}\left\|D_{\mathbb{W}_{i}}\right\|_{2}^{2}$. Therefore, the probability that $G_{T}\left(\mathbb{W}_{i}\right)$ has more than $T^{2}\left(\left\|\mathbb{W}_{i}\right\|_{1}+c^{3}\right) / 2$ or less than $T^{2}\left(\left\|\mathbb{W}_{i}\right\|_{1}-c^{3}\right) / 2$ edges is less than

$$
\frac{T^{2}\left\|\mathbb{W}_{i}\right\|_{1} / 2+T^{3}\left\|D_{\mathbb{W}_{i}}\right\|_{2}^{2}}{c^{6} T^{4} / 4} \leq \frac{2 C+4 T C D}{c^{6} T^{2}} \leq \frac{c}{8} .
$$

Here we used the fact that $\left\|D_{\mathbb{W}_{i}}\right\|_{2}^{2} \leq\left\|D_{\mathbb{W}_{i}}\right\|_{1}\left\|D_{\mathbb{W}_{i}}\right\|_{\infty}=\left\|\mathbb{W}_{i}\right\|_{1}\left\|D_{\mathbb{W}_{i}}\right\|_{\infty}$, and we are assuming that $T$ is large. Assuming neither of these events happens, $\left\|\mathbb{W}_{i}^{\prime}\right\|_{1}$ is between $\left\|\mathbb{W}_{i}\right\|_{1}-c^{3}$ and $\left\|\mathbb{W}_{i}\right\|_{1}+c^{3}$. Since $\left|\left\|\mathbb{W}_{1}\right\|_{1}-\left\|\mathbb{W}_{2}\right\|_{1}\right| \leq c^{3}$, we have that $\left|\left\|\mathbb{W}_{1}^{\prime}\right\|_{1}-\left\|\mathbb{W}_{2}^{\prime}\right\|_{1}\right| \leq 3 c^{3}$.

To summarize, we have that with high probability,

$$
\left.d_{2 \rightarrow 2}\left(\mathbb{W}_{1}^{\prime}, \mathbb{W}_{2}^{\prime}\right) \leq \min (31 c C)^{1 / 4}, 2 c^{3 / 4}, \sqrt[3]{3} c\right) .
$$

The probability that this does not happen is at most

This completes the proof.

$$
\frac{6 c}{10}+\frac{c}{8}+2 \frac{c}{8} \leq c .
$$

7.2. Samples converge to graphex. In this subsection, we prove the sampling lemma, Theorem 7.1. To this end, we will first establish two lemmas. The first one states that each $(C, D)$-bounded graphex can be approximated by a step graphon, i.e., a graphex where the star and dust part is zero, and the graphon part is a step graphon.

Lemma 7.6. For every $\varepsilon, C$, and $D$, there exist $M, N$, and $\rho$ such that the following holds. For every $(C, D)$-bounded graphex $\mathbb{W}$, there exists a $(C, D)$-bounded graphex $\mathbb{W}_{\varepsilon}=\left(W_{\varepsilon}, 0,0, \boldsymbol{\Omega}_{\varepsilon}\right)$, where $\boldsymbol{\Omega}_{\varepsilon}=\left(\Omega_{\varepsilon}, \mathcal{F}_{\varepsilon}, \mu_{\varepsilon}\right)$ and $\mu_{\varepsilon}\left(\Omega_{\varepsilon}\right) \leq N$, and furthermore the graphon $W_{\varepsilon}$ is a step function with at most $M$ steps, with each part having size equal to $\rho$, and $\delta_{2 \rightarrow 2}\left(\mathbb{W}, \mathbb{W}_{\varepsilon}\right) \leq \varepsilon$.

Proof. By Remark 4.4, we may assume that $\mathbb{W}$ is a graphex over an atomless measure space $\boldsymbol{\Omega}=(\Omega, \mathcal{F}, \mu)$. By Theorem 5.7, there exists $M(\varepsilon)$ and $\rho$ such that there is a partition $\mathcal{P}=\left\{P_{1}, P_{2}, \ldots, P_{m}\right\}$ of $\Omega_{\mathscr{P}} \subseteq \Omega$ with $m \leq M(\varepsilon)$ such that $\delta_{2 \rightarrow 2}(\mathbb{W} \mathscr{P}, \mathbb{W}) \leq \varepsilon / 2$ and each part has size $\rho$. Let $\Omega_{\varepsilon}=\Omega_{\mathscr{P}} \cup Q$ where $Q$ is any 
set disjoint from $\Omega_{\mathscr{P}}$, and obtain $\mu_{\varepsilon}$ by extending $\mu$ to $Q$ (with measure to be determined later $)$. Let $\mathbb{W}_{\varepsilon}=\left(W_{\varepsilon}, 0,0, \boldsymbol{\Omega}_{\varepsilon}\right)$ with

$$
W_{\varepsilon}(x, y)= \begin{cases}W_{\mathscr{P}}(x, y) & \text { if } x \in P_{i}, y \in P_{j}, \\ \frac{S_{\mathscr{P}}(x)}{\mu(Q)} & \text { if } x \in P_{i}, y \in Q, \\ \frac{S_{\mathscr{P}}(y)}{\mu(Q)} & \text { if } x \in Q, y \in P_{i}, \text { and } \\ \frac{2 I_{\mathscr{O}}}{\mu(Q)^{2}} & \text { if } x, y \in Q .\end{cases}
$$

Extend $\mathbb{W}_{\mathscr{P}}$ by 0 to $Q$. Since $\mathbb{W}_{\mathscr{P}}$ is $(C, D)$-bounded, there exists $K$ depending only on $\varepsilon, C, D$ such that if $\mu(Q) \geq K$, then $W_{\varepsilon}-W_{\mathscr{P}}$ is at most $\varepsilon^{2} /(4 C)$ everywhere, which implies that

$$
\begin{aligned}
\left\|W_{\varepsilon}-W_{\mathscr{P}}\right\|_{2 \rightarrow 2} & \leq\left\|W_{\varepsilon}-W_{\mathscr{P}}\right\|_{2} \\
& \leq \sqrt{\left\|W_{\varepsilon}-W_{\mathscr{P}}\right\|_{1}\left\|W_{\varepsilon}-W_{\mathscr{P}}\right\|_{\infty}} \leq \sqrt{C \varepsilon^{2} /(4 C)}=\varepsilon / 2 .
\end{aligned}
$$

For $x \in \Omega_{\mathcal{P}}$,

$$
D_{\mathbb{W}_{\varepsilon}}(x)=D_{W_{\mathcal{P}}}(x)+\mu(Q) \frac{S_{\mathcal{P}}(x)}{\mu(Q)}=D_{\mathbb{W}_{\mathcal{P}}}(x) .
$$

We also have that for $x \in Q$,

$$
D_{\mathbb{W}_{\varepsilon}}(x)=\mu(Q) \frac{2 I_{\mathscr{P}}}{\mu(Q)^{2}}+\sum_{i} \int_{P_{i}} \frac{S_{\mathscr{P}}(y)}{\mu(Q)} d \mu(y)=\frac{D_{W_{\mathscr{P}}}(\infty)}{\mu(Q)} .
$$

Therefore, there exists a $K^{\prime}$ depending only on $\varepsilon, C$, and $D$ such that if $\mu(Q) \geq K^{\prime}$, then

$$
\begin{aligned}
\left\|D_{\mathbb{W}_{\varepsilon}}-D_{\mathbb{W}_{\mathscr{P}}}\right\|_{2}^{2} & =\int_{\Omega_{\mathscr{P}} \cup Q}\left(D_{\mathbb{W}_{\varepsilon}}(x)-D_{\mathbb{W}_{\mathscr{P}}}(x)\right)^{2} d \mu(x) \\
& =\int_{Q}\left(\frac{D_{\mathbb{W}_{\mathscr{P}}}(\infty)}{\mu(Q)}\right)^{2}=\frac{D_{\mathbb{W}_{\mathscr{P}}}(\infty)^{2}}{\mu(Q)} \leq \varepsilon^{4} / 16 .
\end{aligned}
$$

Also, by construction, $\left\|\mathbb{W}_{\varepsilon}\right\|_{1}=\left\|\mathbb{W}_{\mathscr{P}}\right\|_{1}$. Therefore $\delta_{2 \rightarrow 2}\left(\mathbb{W}_{\varepsilon}, \mathbb{W}_{\mathscr{P}}\right) \leq \varepsilon / 2$, and hence $\delta_{2 \rightarrow 2}\left(\mathbb{W}_{\varepsilon}, \mathbb{W}\right) \leq \varepsilon$.

Remark 7.7. Using the ideas of the previous proof, it is not hard to see that in distribution, the process generated from the graphex $\widetilde{\mathbb{W}}_{Q}=\left(\widetilde{W}_{Q}, 0,0, \widetilde{\boldsymbol{\Omega}}_{D}\right)$ constructed in Remark 2.3 (3) converges to the one generated from $\mathbb{W}$. Indeed, we claim that

$$
\delta_{\diamond}\left(\widetilde{\mathbb{W}}_{Q}, \mathbb{W}\right) \rightarrow 0 \quad \text { as } \quad Q \rightarrow \infty .
$$

To see this, fix $\varepsilon>0$ and choose $D$ in such a way that the set $\Omega_{>D}=\left\{D_{\mathbb{W}}>D\right\}$ has measure at most $\varepsilon^{2}$. Let $\Omega_{\leq D}=\Omega \backslash \Omega_{>D}$ and $\widetilde{\Omega}_{\leq D}=\Omega_{\leq D} \cup\{\infty\}=\widetilde{\Omega} \backslash \Omega_{>D}$. Setting $\widetilde{\mathbb{W}}_{Q, \leq D}=\left(\widetilde{\mathbb{W}}_{Q}\right)_{\mid \widetilde{\Omega} \backslash \Omega_{>D}}$ and $\mathbb{W}_{\leq D}=\mathbb{W}_{\mid \Omega \backslash \Omega>D}$ and defining $\widetilde{\mathbb{W}}_{\leq D}$ as the trivial extension of $\mathbb{W}_{\leq D}$ to $\widetilde{\Omega}_{\leq D}$, we will want to show that for $Q$ large enough, $d_{2 \rightarrow 2}\left(\widetilde{\mathbb{W}}_{\leq D}, \widetilde{\mathbb{W}}_{Q, \leq D}\right) \leq \varepsilon$, since this implies that $\delta_{2 \rightarrow 2}\left(\mathbb{W}_{\leq D}, \widetilde{\mathbb{W}}_{Q, \leq D}\right) \leq \varepsilon$ and hence $\delta_{\diamond}\left(\widetilde{\mathbb{W}}_{Q}, \mathbb{W}\right) \leq \varepsilon$. But this follows by essentially the same argument as the one in the previous proof; all that is needed is that by Proposition 2.4, $\widetilde{\mathbb{W}}_{\leq D}$ is $(C, D)$-bounded for some $C<\infty$.

Our second lemma estimates the distance between the empirical graphex corresponding to a weighted graph $H$ with weights in $[0,1]$ and the one corresponding to the graph $G$ obtained from $H$ by choosing the edge in $G$ randomly according 
to $H$. More precisely, given a finite weighted graph $H$ with weights $H_{i, j} \in[0,1]$ and $H_{i, i}=0$, define $G(H)$ as the graph generated by taking an edge between $i$ and $j$ with probability $H_{i, j}$, independently for every pair of vertices. Our next lemma estimates the distance between the empirical graphon of $H$ and the empirical graphon of $G(H)$.

Lemma 7.8. For every $N_{0}, \varepsilon$, and $\delta$, there exists $n_{0}$ such that the following holds. For any weighted graph $H$ on $n \geq n_{0}$ vertices with weights in $[0,1]$, and any $N \leq N_{0}$, the probability that $\delta_{2 \rightarrow 2}(\mathbb{W}(H, N / n), \mathbb{W}(G(H), N / n))>\varepsilon$ is at most $\delta$.

Proof. We first extend both $H$ and $G$ trivially to $\mathbb{N}$, and then define $U$ as the graphon $U=W(G(H), N / n)-W(H, N / n)$. Then $\|U\|_{2 \rightarrow 2} \leq\left(t\left(C_{4}, U\right)\right)^{1 / 4}$ by Lemma 3.18. As a consequence, the probability that $\|U\|_{2 \rightarrow 2}>\varepsilon$ is bounded by $\varepsilon^{-4} \mathbb{E}\left[t\left(C_{4}, U\right)\right]$. Using the bound (7.1) from the proof of Lemma 7.5 with $E=n^{2}$, $F=n^{3}$, and $\rho=N / n$, we get that the probability that $\|U\|_{2 \rightarrow 2}>\varepsilon$ is bounded by

$$
\varepsilon^{-4} \mathbb{E}\left[t\left(C_{4}, U\right)\right] \leq \varepsilon^{-4} \frac{N^{4}}{n^{4}}\left(n^{2}+2 n^{3}\right) \leq 3 \varepsilon^{-4} n^{3} \frac{N^{4}}{n^{4}} \leq 3 \varepsilon^{-4} \frac{N_{0}^{4}}{n_{0}} \leq \delta / 2,
$$

provided $n_{0} \geq 6 \varepsilon^{-4} \delta^{-1} N_{0}^{4}$. Let us now bound the other two components of $\Delta_{2 \rightarrow 2}$. For a fixed vertex $v$, by Hoeffding's inequality [15],

$$
\mathbb{P}\left[\left|d_{G(H)}(v)-d_{H}(v)\right|>\varepsilon^{\prime} n\right] \leq 2 e^{-2 \varepsilon^{\prime 2} n} .
$$

Therefore, by a union bound,

$$
\mathbb{P}\left[\text { there exists a vertex } v \text { such that }\left|d_{G(H)}(v)-d_{H}(v)\right|>\varepsilon^{\prime} n\right] \leq 2 n e^{-2 \varepsilon^{\prime 2} n} .
$$

For any fixed $\varepsilon^{\prime}$, if $n$ is large enough, this probability is less than $\delta / 2$. If this does not happen, then for every vertex $v$,

$$
\left|D_{W\left(G(H), \frac{N}{n}\right)}(v)-D_{W\left(H, \frac{N}{n}\right)}(v)\right| \leq N \varepsilon^{\prime} .
$$

Therefore, for $\varepsilon^{\prime}$ small enough,

$$
\left\|D_{W(G(H), N / n)}-D_{W(H, N / n)}\right\|_{2} \leq N^{3 / 2} \varepsilon^{\prime} \leq \varepsilon^{2},
$$

and

$$
\begin{aligned}
\left|\|W(G(H), N / n)\|_{1}-\|W(H, N / n)\|_{1}\right| & =\left|\left\|D_{W(G(H), N / n)}\right\|_{1}-\left\|D_{W(H, N / n)}\right\|_{1}\right| \\
& \leq N^{2} \varepsilon^{\prime} \leq \varepsilon^{3} .
\end{aligned}
$$

This completes the proof of the lemma.

With these preparations, we are ready to prove the sampling lemma.

Proof of Theorem 7.1. Fix $\varepsilon>0$. We know from the definition of tightness that there exist $C$ and $D$ so that we can remove a set $\Omega_{\varepsilon}$ of measure at most $\varepsilon^{2} / 2$ to obtain a $(C, D)$-bounded graphex. Then the expected number of points in $G_{T}$ whose feature lies inside $\Omega_{\varepsilon}$ is $\varepsilon^{2} T / 2$. Therefore, since it is a Poisson distribution, the probability that $G_{T}(\mathbb{W})$ has more than $\varepsilon^{2} T$ points in $\Omega_{\varepsilon}$ is at most

$$
e^{\frac{\varepsilon^{2} T}{2}(1-2 \log 2)} \text {. }
$$

This converges to 0 as $T \rightarrow \infty$. If $G_{T}(\mathbb{W})$ does not have more than $\varepsilon^{2} T$ points, then we can remove those points from $G_{T}(\mathbb{W})$ and the sample is equivalent to a sample from the graphex restricted to $\Omega \backslash \Omega_{\varepsilon}$. (It may have isolated vertices but this does not affect our distance.) Since a set of $\varepsilon^{2} T$ points in $G_{T}(\mathbb{W})$ corresponds to a set 
of measure $\varepsilon^{2}$ in $\mathbb{W}\left(G_{T}(\mathbb{W}, 1 / T)\right)$, this shows that we may assume without loss of generality that the original set is $(C, D)$-bounded, and prove Theorem 7.1 for $\delta_{2 \rightarrow 2}$ instead of $\delta_{\diamond}$.

Choose $\mathbb{W}_{\delta}$ as in Lemma 7.6 (with $\delta$ taking the role of $\varepsilon$ ) so that in particular $\delta_{2 \rightarrow 2}\left(\mathbb{W}, \mathbb{W}_{\delta}\right) \leq \delta$. For sufficiently small $\delta$, Theorem 7.3 then implies that there exists a $T_{0}$ such that if $T>T_{0}$, then the samples from $\mathbb{W}$ and $\mathbb{W}_{\delta}$ can be coupled so that

$$
\mathbb{P}\left[\delta_{2 \rightarrow 2}\left(\mathbb{W}\left(G_{T}(\mathbb{W}), 1 / T\right), \mathbb{W}\left(G_{T}\left(\mathbb{W}_{\delta}\right), 1 / T\right)\right)>(31 C \delta)^{1 / 4}\right]<\delta
$$

This means that it suffices to prove Theorem 7.1 for step function graphons with equal size parts, uniformly over any set of graphons with a bounded number of parts with the same size. Indeed, for any $\varepsilon>0$, let $\delta>0$ be such that

$$
2 \delta+(31 \delta C)^{1 / 4}<\varepsilon \text {. }
$$

If we then take $\mathbb{W}_{\delta}$ as above, then $\delta_{2 \rightarrow 2}\left(\mathbb{W}_{\delta}, \mathbb{W}\right) \leq \delta$, so by Theorem 7.3 , for large enough $T$, we can couple $\mathbb{W}\left(G_{T}\left(\mathbb{W}_{\delta}, 1 / T\right)\right)$ and $\mathbb{W}\left(G_{T}(\mathbb{W}, 1 / T)\right)$ so that the probability that they have $\delta_{2 \rightarrow 2}$ distance more than $(31 \delta C)^{1 / 4}$ is at most $\delta$. Furthermore, we can take $T$ large enough so that the probability that $\delta_{2 \rightarrow 2}\left(\mathbb{W}\left(G_{T}\left(\mathbb{W}_{\delta}\right), 1 / T\right), \mathbb{W}_{\delta}\right)>\delta$ is at most $\delta$ (detailed below). Overall, by the triangle inequality, this implies that the probability that $\delta_{2 \rightarrow 2}\left(\mathbb{W}\left(G_{T}(\mathbb{W}), 1 / T\right), \mathbb{W}\right) \geq \varepsilon$ is at most $2 \delta$. Since this works for arbitrarily small $\delta$, the theorem follows.

Suppose therefore that $\mathbb{W}=(W, 0,0, \boldsymbol{\Omega})$, where $W$ is a step graphon with step size $\rho$ and $m$ steps total. Fix $\varepsilon>0$ and $\delta>0$. For a fixed part $P_{i}$ and $T$, let $X_{T, i}$ be the number of points in $P_{i}$ in the Poisson process. The expectation of each $X_{T, i}$ is $\rho T$. For $\varepsilon^{\prime}>0$, we have

$$
\mathbb{P}\left[X_{T, i}>\left(1+\varepsilon^{\prime}\right) \rho T\right]<e^{\rho T\left(\varepsilon^{\prime}-\left(1+\varepsilon^{\prime}\right) \log \left(1+\varepsilon^{\prime}\right)\right)}=e^{-\rho T c\left(\varepsilon^{\prime}\right)}
$$

for a nonnegative number $c\left(\varepsilon^{\prime}\right)$. We also have

$$
\mathbb{P}\left[X_{T, i}<\left(1-\varepsilon^{\prime}\right) \rho T\right]<e^{\rho T\left(-\varepsilon^{\prime}+\left(1-\varepsilon^{\prime}\right) \log \left(\frac{1}{1-\varepsilon^{\prime}}\right)\right.}=e^{-\rho T c^{\prime}\left(\varepsilon^{\prime}\right)}
$$

for a nonnegative number $c^{\prime}\left(\varepsilon^{\prime}\right)$. Therefore, if $T$ is large enough, then the probability that any part $P_{i}$ has more than $\left(1+\varepsilon^{\prime}\right) \rho T$ or less than $\left(1-\varepsilon^{\prime}\right) \rho T$ points is less than $\delta / 2$. Note that in particular this means that the total measure of nonzero points is at most $\left(1+\varepsilon^{\prime}\right) \rho m$. Therefore, with probability at least $1-\delta / 2$, we can add or delete points with total measure at most $\varepsilon^{\prime} \rho m$ to obtain $W$ from $W\left(H_{T}(W), \rho\right)$. This means that we can couple $\mathbb{W}\left(H_{T}(W), \rho\right)$ and $\mathbb{W}$ so that they differ on points with total measure at most $\varepsilon^{\prime} \rho m$, and hence

$$
\left\|W\left(H_{T}(W), \rho\right)-W\right\|_{1} \leq 2 \varepsilon^{\prime}\left(1+\varepsilon^{\prime}\right) \rho^{2} m^{2} .
$$

Therefore, we have the same bound for $\left|\left\|W\left(H_{T}(W), \rho\right)\right\|_{1}-\|W\|_{1}\right|$. Since both graphons are between 0 and 1 , we also have

$$
\begin{aligned}
\left\|W\left(H_{T}(W), \rho\right)-W\right\|_{2 \rightarrow 2} & \leq\left\|W\left(H_{T}(W), \rho\right)-W\right\|_{2} \\
& \leq \sqrt{\left\|W\left(H_{T}(W), \rho\right)-W\right\|_{1}} \leq \sqrt{2 \varepsilon^{\prime}\left(1+\varepsilon^{\prime}\right)} \rho m .
\end{aligned}
$$

Finally, we have

$$
\left\|D_{\mathbb{W}\left(H_{T}(W), \rho\right)}-D_{\mathbb{W}}\right\|_{2}^{2} \leq \rho m\left(\varepsilon^{\prime} \rho m\right)^{2}+\varepsilon^{\prime} \rho m\left(\left(1+\varepsilon^{\prime}\right) \rho m\right)^{2}=\left({\varepsilon^{\prime}}^{3}+3{\varepsilon^{\prime}}^{2}+\varepsilon^{\prime}\right) \rho^{3} m^{3} .
$$

We can therefore take $\varepsilon^{\prime}$ small enough that $\delta_{2 \rightarrow 2}\left(\mathbb{W}\left(H_{T}(W), \rho\right)\right.$, W $)<\varepsilon / 2$ with probability at least $1-\delta / 2$. 
Using Lemma 7.8 for $\varepsilon / 2$ and $\delta / 2$, we have that with probability at least $1-\delta / 2$,

$$
\delta_{2 \rightarrow 2}\left(\mathbb{W}, H_{T}(W, 1 / T)\right) \leq \frac{\varepsilon}{2},
$$

and with probability at least $1-\delta / 2$,

$$
\delta_{2 \rightarrow 2}\left(\mathbb{W}\left(H_{T}(W), 1 / T\right), \mathbb{W}\left(G_{T}(\mathbb{W}), 1 / T\right)\right) \leq \frac{\varepsilon}{2} .
$$

Therefore, with probability at least $1-\delta$,

$$
\begin{aligned}
\delta_{2 \rightarrow 2}(\mathbb{W}, & \left.\mathbb{W}\left(G_{T}(\mathbb{W}), 1 / T\right)\right) \\
& \leq \delta_{2 \rightarrow 2}\left(\mathbb{W}, H_{T}(W, 1 / T)\right)+\delta_{2 \rightarrow 2}\left(\mathbb{W}\left(H_{T}(W), 1 / T\right), \mathbb{W}\left(G_{T}(\mathbb{W}), 1 / T\right)\right) \\
& \leq \frac{\varepsilon}{2}+\frac{\varepsilon}{2}=\varepsilon .
\end{aligned}
$$

This completes the proof of Theorem 7.1.

7.3. Proofs of Theorem 2.18, Proposition 2.13, and Theorem 2.23. Having completed the proof of Theorem 7.1, we are finally ready to establish that $\delta_{\diamond}$ convergence is equivalent to GP-convergence, together with several of the other equivalences stated in Section 2. To this end, we first prove the following theorem.

Theorem 7.9. Given a pair of graphexes $\mathbb{W}, \mathbb{W}^{\prime}$, we have $\delta_{\diamond}\left(\mathbb{W}, \mathbb{W}^{\prime}\right)=0$ if and only if for every $T>0, G_{T}(\mathbb{W})$ and $G_{T}\left(\mathbb{W}^{\prime}\right)$ have the same distribution.

Proof. If $\delta_{\diamond}\left(\mathbb{W}^{\prime}, \mathbb{W}\right)=0$, then taking $\mathbb{W}_{n}=W^{\prime}$ for each $n$, Theorem 6.17 implies that $G_{T}(\mathbb{W})$ and $G_{T}\left(\mathbb{W}^{\prime}\right)$ must have the same distribution for every $T$. Suppose now that $G_{T}(\mathbb{W})$ and $G_{T}\left(\mathbb{W}^{\prime}\right)$ have the same distribution for every $T$. By Theorem 7.1, we can choose $T$ such that with probability at least $0.99, \delta_{\diamond}\left(G_{T}(\mathbb{W}), \mathbb{W}\right)<\varepsilon / 2$ and $\delta_{\diamond}\left(G_{T}\left(\mathbb{W}^{\prime}\right), \mathbb{W}^{\prime}\right)<\varepsilon / 2$. Since $G_{T}(\mathbb{W})$ and $G_{T}\left(\mathbb{W}^{\prime}\right)$ have the same distribution, the two graphexes have distance at most $\varepsilon$. Since this holds for every $\varepsilon$, the lemma follows.

Proof of Theorems 2.18, Proposition 2.13, and Theorem 2.23. We start with the proof of Theorem 2.18. One direction follows from Theorem 6.17. Suppose now that $\mathbb{W}_{n}$ is GP-convergent to $\mathbb{W}$. We know by Theorem 4.1 that then the set $\mathbb{W}_{n}$ is tight. By Theorem 2.21, $\mathbb{W}_{n}$ therefore has a subsequence that converges according to $\delta_{\diamond}$ to a graphex $\mathbb{W}^{\prime}$, which in turn implies the subsequence is GP-convergent to $\mathbb{W}^{\prime}$. This implies that for any $T>0, G_{T}(\mathbb{W})$ and $G_{T}\left(\mathbb{W}^{\prime}\right)$ have the same distribution. By Theorem $7.9, \delta_{\diamond}\left(\mathbb{W}, \mathbb{W}^{\prime}\right)=0$, so $\delta_{\diamond}\left(\mathbb{W}_{n}, \mathbb{W}\right) \rightarrow 0$. Next recall that by Proposition 2.17, the distances $\delta_{\diamond}$ and $\delta_{2 \rightarrow 2}$ give equivalent topologies on sets with uniformly bounded marginals, showing that Theorem 2.18 implies Proposition 2.13. We conclude by noting that Theorem 2.23 follows from Corollary 6.2 and Proposition 2.13.

\section{IDENTIFIABILITY}

In this section, we prove Theorem 2.5. In fact, we will prove the following version, which by Theorem 7.9 is equivalent.

Theorem 8.1. Let $\mathbb{W}_{1}=\left(W_{1}, S_{1}, I_{1}, \boldsymbol{\Omega}_{1}\right)$ and $\mathbb{W}_{2}=\left(W_{2}, S_{2}, I_{2}, \boldsymbol{\Omega}_{2}\right)$ be graphexes, where $\boldsymbol{\Omega}_{i}=\left(\Omega_{i}, \mathcal{F}_{i}, \mu_{i}\right)$ are $\sigma$-finite spaces. Suppose $\delta_{\diamond}\left(\mathbb{W}_{1}, \mathbb{W}_{2}\right)=0$. Then there exists a third graphex $\mathbb{W}=(W, S, I, \boldsymbol{\Omega})$ over a $\sigma$-finite measure space $\boldsymbol{\Omega}=(\Omega, \mathcal{F}, \mu)$ and measure preserving maps $\phi_{i}: \operatorname{dsupp} W_{i} \rightarrow \Omega$ such that $\left.\mathbb{W}_{i}\right|_{\phi^{-1}(\Omega)}=\mathbb{W}^{\phi_{i}}$ (and $W_{i}, S_{i}=0$ everywhere else) for $i=1,2$. 
To prove the theorem, we will first prove the following theorem, which may be of independent interest. We recall that a Borel measure space is a measure space that is isomorphic to a Borel subset of a complete separable metric space equipped with a Borel measure, where, as usual, two measure spaces $\boldsymbol{\Omega}=(\Omega, \mathcal{F}, \mu)$ and $\boldsymbol{\Omega}=(\Omega, \mathcal{F}, \mu)$ are called isomorphic if there exists a bijective map $\phi: \Omega \rightarrow \Omega^{\prime}$ such that both $\phi$ and its inverse are measure preserving.

Theorem 8.2. Let $\mathbb{W}_{1}=\left(W_{1}, S_{1}, I_{1}, \boldsymbol{\Omega}_{1}\right)$ and $\mathbb{W}_{2}=\left(W_{2}, S_{2}, I_{2}, \boldsymbol{\Omega}_{2}\right)$ be graphexes, where $\boldsymbol{\Omega}_{i}=\left(\Omega_{i}, \mathcal{F}_{i}, \mu_{i}\right)$ are $\sigma$-finite Borel spaces. Suppose further that $D_{\mathbb{W}_{i}}>0$ everywhere for $i=1,2$, and $\delta_{\diamond}\left(\mathbb{W}_{1}, \mathbb{W}_{2}\right)=0$. Then $\mu_{1}\left(\Omega_{1}\right)=\mu_{2}\left(\Omega_{2}\right), I_{1}=I_{2}$, and there exists a coupling of $\boldsymbol{\Omega}_{1}$ and $\boldsymbol{\Omega}_{2}$, that is, a measure $\nu$ on $\left(\Omega_{1} \times \Omega_{2}, \mathcal{F}_{1} \times \mathcal{F}_{2}\right)$ with marginals $\mu_{1}$ and $\mu_{2}$, such that if $\pi_{i}: \Omega_{1} \times \Omega_{2} \rightarrow \Omega_{i}$ is the projection map, then $W_{1}^{\pi_{1}}=W_{2}^{\pi_{2}} \nu$-almost-everywhere, and $S_{1}^{\pi_{1}}=S_{2}^{\pi_{2}} \nu$-almost-everywhere.

Theorem 8.2 should be compared to Proposition 8 from [3] which states the analogous result for integrable Borel graphons that have cut distance zero (without the assumption that $W_{1}$ and $W_{2}$ are non-negative), using a different proof technique. Using still different proof techniques, Janson proved a similar result (again without assuming non-negativity), showing that after trivially extending two integrable Borel graphons with cut distance zero they can be coupled so that the projections are equal almost everywhere; see [17]. We will prove Theorem 8.2 in Section 8.1.

Remark 8.3. Throughout this paper, we have considered graphexes where all three parts are non-negative. While this makes sense when considering graphexes as generators of a graphex process, from an analytical point of view, it is less natural. Indeed, it is easy to define the kernel and weak kernel distance for graphexes where the three parts take values in $\mathbb{R}$. Taking, e.g., the kernel distance $d_{2 \rightarrow 2}$ defined in (2.1), all we need to do is replace the $L^{1}$ norms in the third part by a signed "edge density" $\rho\left(\mathbb{W}_{i}\right)=\int W d \mu \times d \mu+2 \int S d \mu+2 I$, and then use the third root of $\left|\rho\left(\mathbb{W}_{1}\right)-\rho\left(\mathbb{W}_{2}\right)\right|$ instead of the third root of $\left|\left\|\mathbb{W}_{1}\right\|_{1}-\left\|\mathbb{W}_{2}\right\|\right|$. In particular in view of the just discussed results from [3] and [17], we conjecture that Theorem 8.2 holds for signed graphexes as well, provided the condition $D_{\mathbb{W}_{i}}>0$ is replaced by the condition $D_{\left|\mathbb{W}_{i}\right|}>0$, where $\left|\mathbb{W}_{i}\right|$ is obtained from $\mathbb{W}_{i}$ by replacing all three components of $\mathbb{W}_{i}$ by their absolute values. We leave the proof of this conjecture as an open problem.

Once we have established Theorem 8.2 , we will then prove Theorem 8.1 by generalizing a construction which was developed by Janson for the dense case in [16]. To this end, we will assign to each graphex $\mathbb{W}$ a "canonical version" $\widehat{\mathbb{W}}$ such that $\mathbb{W}$ is a pullback of $\widehat{\mathbb{W}}$ and show that if two graphexes are equivalent, then their canonical versions are isomorphic up to measure zero changes. This will be carried out in Section 8.2.

Remark 8.4. Section 8.2 does not use nonnegativity in any essential way, and should easily generalizable to signed graphexes. This should give a relatively straightforward proof of the analogue of Theorem 8.1 for graphons of cut distance zero, and also allow for the more general setting of signed graphexes, once the above conjectured generalization of Theorem 8.2 is established. Again we leave this as an open problem.

8.1. Infimum is minimum. In this subsection, we will prove Theorem 8.2. The proof will be based on a series of lemmas. 
BORGS, CHAYES, COHN, AND LOVÁSZ

Let $\widetilde{\mathbb{W}}_{i}=\left(\widetilde{W}_{i}, \widetilde{S}_{i}, \widetilde{I}_{i}, \widetilde{\Omega}_{i}\right)$, for $i=1,2$, be trivial extensions of $\mathbb{W}_{i}$ to spaces of infinite measure, where $\widetilde{\Omega}_{i}=\left(\widetilde{\Omega}_{i}, \widetilde{\mathcal{F}}_{i}, \widetilde{\mu}_{i}\right)$, and let $\varepsilon>0$. By Proposition 4.7 (3), there exist $\widetilde{\Omega}_{i}^{\varepsilon} \subseteq \widetilde{\Omega}_{i}$ such that $\widetilde{\Omega}_{i} \backslash \widetilde{\Omega}_{i}^{\varepsilon}$ has measure at most $\varepsilon$, and a measure $\nu_{\varepsilon}$ on $\widetilde{\Omega}_{1}^{\varepsilon} \times \widetilde{\Omega}_{2}^{\varepsilon}$ with marginals $\left.\widetilde{\mu}_{1}\right|_{\widetilde{\Omega}_{1}^{\varepsilon}}$ and $\left.\widetilde{\mu}_{2}\right|_{\widetilde{\Omega}_{2}^{\varepsilon}}$ such that for the restricted graphexes $\widetilde{\mathbb{W}}_{i, \varepsilon}$,

$$
\left\|\widetilde{W_{1, \varepsilon}^{\pi_{1}}}-\widetilde{W_{2, \varepsilon}^{\pi_{2}}}\right\|_{2 \rightarrow 2, \nu_{\varepsilon}} \leq \varepsilon
$$

and

$$
\int_{\widetilde{\Omega}_{1}^{\varepsilon} \times \widetilde{\Omega}_{2}^{\varepsilon}}\left(D_{\widetilde{\mathbb{W}}_{1, \varepsilon}}(x)-D_{\widetilde{\mathbb{W}}_{2, \varepsilon}}(y)\right)^{2} d \nu_{\varepsilon}(x, y) \leq \varepsilon^{4} .
$$

With a slight abuse of notation, we extend $\nu_{\varepsilon}$ to $\widetilde{\Omega}_{1} \times \widetilde{\Omega}_{2}$ by zero. This means that in fact

and

$$
\left\|\widetilde{W}_{1}^{\pi_{1}}-\widetilde{W}_{2}^{\pi_{2}}\right\|_{2 \rightarrow 2, \nu_{\varepsilon}} \leq \varepsilon
$$

We first prove the following lemma.

Lemma 8.5. For any $c$,

(1) $\lim _{\varepsilon \rightarrow 0} \nu_{\varepsilon}\left(\Omega_{1,>c} \times \Omega_{2,>c}\right)=\mu_{i}\left(\Omega_{i,>c}\right)$ for $i=1,2$ (regardless of the choice of $\left.\nu_{\varepsilon}\right)$, and

(2) $\mu_{1}\left(\Omega_{1,>c}\right)=\mu_{2}\left(\Omega_{2,>c}\right)$.

Proof. We first prove (1). By symmetry, it suffices to prove it for $i=1$. Note that for any $x \in \widetilde{\Omega}_{i}^{\varepsilon}$,

$$
D_{\widetilde{\mathbb{W}}_{i, \varepsilon}}(x) \leq D_{\widetilde{\mathbb{W}}_{i}}(x) \leq D_{\widetilde{\mathbb{W}}_{i, \varepsilon}}(x)+\varepsilon
$$

and that $\widetilde{\Omega}_{i,>c}=\Omega_{i,>c}$. Therefore,

$$
\begin{aligned}
\varepsilon \nu_{\varepsilon}\left(\Omega_{1,>c} \times\left(\widetilde{\Omega}_{2} \backslash\right.\right. & \left.\left.\Omega_{2,>c-(\sqrt{\varepsilon}+\varepsilon)}\right)\right) \\
\leq & \int_{\Omega_{1,>c} \times\left(\widetilde{\Omega}_{2} \backslash \Omega_{2,>c-(\sqrt{\varepsilon}+\varepsilon)}\right)}\left(D_{\mathbb{W}_{1, \varepsilon}}(x)-D_{\mathbb{W}_{2, \varepsilon}}(y)\right)^{2} \leq \varepsilon^{4},
\end{aligned}
$$

which implies that

$$
\nu_{\varepsilon}\left(\Omega_{1,>c} \times\left(\widetilde{\Omega}_{2} \backslash \Omega_{2,>c-(\sqrt{\varepsilon}+\varepsilon)}\right)\right)<\varepsilon^{3} .
$$

Now, for any $\varepsilon$,

$$
\begin{aligned}
& \left|\mu_{1}\left(\Omega_{1,>c}\right)-\nu_{\varepsilon}\left(\Omega_{1,>c} \times \Omega_{2,>c}\right)\right| \leq \varepsilon+\left|\nu_{\varepsilon}\left(\Omega_{1,>c} \times \widetilde{\Omega}_{2}\right)-\nu_{\varepsilon}\left(\Omega_{1,>c} \times \Omega_{2,>c}\right)\right| \\
& \quad=\varepsilon+\nu_{\varepsilon}\left(\Omega_{1,>c} \times\left(\widetilde{\Omega}_{2} \backslash \Omega_{2,>c}\right)\right) \\
& \quad=\varepsilon+\nu_{\varepsilon}\left(\Omega_{1,>c} \times\left(\widetilde{\Omega}_{2} \backslash \Omega_{2,>c-(\sqrt{\varepsilon}+\varepsilon)}\right)\right)+\nu_{\varepsilon}\left(\Omega_{1,>c} \times\left(\Omega_{\left.\left.2,>c-(\sqrt{\varepsilon}+\varepsilon) \backslash \Omega_{2,>c}\right)\right)}\right.\right. \\
& \quad<\varepsilon+\varepsilon^{3}+\mu_{2}\left(\Omega_{2,>c-(\sqrt{\varepsilon}+\varepsilon)} \backslash \Omega_{2,>c}\right) .
\end{aligned}
$$

This last expression is finite, and tends to 0 as $\varepsilon \rightarrow 0$, so

$$
\lim _{\varepsilon \rightarrow 0} \nu_{\varepsilon}\left(\Omega_{1,>c} \times \Omega_{2,>c}\right)=\mu_{1}\left(\Omega_{1,>c}\right) .
$$

This proves (1). From this, (2) is obvious.

Lemma 8.6. For any $n \geq 1$, there exists a measure $\nu_{n}$ on $\Omega_{1,>1 / n} \times \Omega_{2,>1 / n}$ such that the following hold:

(1) $\nu_{n}$ is a coupling of $\Omega_{1,>1 / n}$ and $\Omega_{2,>1 / n}$, 
(2) $\left.\nu_{n+1}\right|_{\Omega_{1,>1 / n} \times \Omega_{2,>1 / n}}=\nu_{n}$,

(3) $W_{1}^{\pi_{1}}$ and $W_{2}^{\pi_{2}}$ are equal when restricted to $\left(\Omega_{1,>1 / n} \times \Omega_{2,>1 / n}\right)^{2}, \nu_{n} \times \nu_{n^{-}}$almost everywhere, and

(4) $D_{\mathbb{W}_{1}^{\pi_{1}}}$ and $D_{\mathbb{W}_{2}^{\pi_{2}}}$ are equal when restricted to $\Omega_{1,>1 / n} \times \Omega_{2,>1 / n}, \nu_{n}$-almost everywhere.

Proof. It is well known that any two Borel measurable spaces with the same cardinality are isomorphic; see, e.g., Theorem 8.3.6 in [12]. As a consequence, each Borel measure space $(\Omega, \mathcal{F}, \mu)$ with $\mu(\Omega)<\infty$ is either empty or isomorphic to a finite set (with the discrete topology), the countable set $\{0\} \cup\{1 / n: n \in \mathbb{N}\}$ (with the induced topology from $\mathbb{R}$ ), or the Cantor cube $\mathcal{C}=\{0,1\}^{\infty}$ (with the product topology), equipped with the Borel $\sigma$-algebras generated by the topologies, and a measure that is a finite Borel measure with full support.

We can therefore assume without loss of generality that for each $i, \Omega_{i,>1}$ and each set $\Omega_{i,>1 /(n+1)} \backslash \Omega_{i,>1 / n}$ are of this form. This means we may without loss of generality assume the following properties:

(1) Each $\Omega_{i,>1 / n}$, and thus each $\Omega_{i,>1 / n}^{2}$, is compact.

(2) For any $i_{1}, i_{2}=1,2$ and $n_{1}, n_{2} \in \mathbb{N}^{+}$, and any finite Borel measure $\nu$ on $\Omega_{i_{1},>1 / n_{1}} \times \Omega_{i_{2},>1 / n_{2}}$, the set of all step functions on $\Omega_{i_{1},>1 / n_{1}} \times \Omega_{i_{2},>1 / n_{2}}$ corresponding to partitions of $\Omega_{i_{j},>1 / n_{j}}$ into clopen sets for $j=1,2$ is dense in $L^{1}\left(\Omega_{i_{1},>1 / n_{1}} \times \Omega_{i_{2},>1 / n_{2}}\right)$.

Now, take a sequence $\varepsilon_{k} \rightarrow 0$, and recall that we have an almost coupling measure $\nu_{\varepsilon_{k}}$ on $\widetilde{\Omega}_{1} \times \widetilde{\Omega}_{2}$ with

and

$$
\left\|\widetilde{W}_{1}^{\pi_{1}}-\widetilde{W}_{2}^{\pi_{2}}\right\|_{2 \rightarrow 2, \nu_{\varepsilon_{k}}} \leq \varepsilon_{k}
$$

$$
\left\|D_{\widetilde{\mathbb{W}}_{1}^{\pi_{1}, \nu_{\varepsilon}}}-D_{\widetilde{\mathbb{W}}_{2}^{\pi_{2}, \nu_{\varepsilon_{k}}}}\right\|_{2} \leq \varepsilon_{k}^{2}
$$

We know that for each $n, \Omega_{1,>1 / n} \times \Omega_{2,>1 / n}$ is compact, and for any $K>0$, the set of measures on it bounded by $K$ is compact under the topology of weak convergence of measures. Since for any $c$,

$$
\lim _{k \rightarrow \infty} \nu_{\varepsilon_{k}}\left(\Omega_{1,>c} \times \Omega_{2,>c}\right)=\mu_{1}\left(\Omega_{1,>c}\right)=\mu_{2}\left(\Omega_{2,>c}\right)<\infty,
$$

we can take a subsequence of $\nu_{\varepsilon_{k}}$ such that for each $n$, the measure is convergent when restricted to $\Omega_{1,>1 / n} \times \Omega_{2,>1 / n}$. Without loss of generality we assume that the original sequence has this property. For each $n$, we then define $\nu_{n}$ as the limit measure on $\Omega_{1,1 / n} \times \Omega_{2,>1 / n}$. Having defined $\nu_{n}$ we now prove (1)-(4).

(1) We have seen in Lemma 8.5 that

$$
\nu_{n}\left(\Omega_{1,>1 / n} \times \Omega_{2,>1 / n}\right)=\mu_{1}\left(\Omega_{1,>1 / n}\right)=\mu_{2}\left(\Omega_{2,>1 / n}\right) .
$$

For any clopen $F \subseteq \Omega_{1,>1 / n}, F \times \Omega_{2,>1 / n}$ is clopen in $\Omega_{1,>1 / n} \times \Omega_{2,>1 / n}$. Therefore,

$$
\lim _{k \rightarrow \infty} \nu_{\varepsilon_{k}}\left(F \times \Omega_{2,>1 / n}\right)=\nu_{n}\left(F \times \Omega_{2,>1 / n}\right) .
$$

On the other hand, by Lemma 8.5,

$$
\nu_{\varepsilon_{k}}\left(F \times\left(\widetilde{\Omega}_{2} \backslash \Omega_{2,>1 / n}\right)\right) \leq \nu_{\varepsilon_{k}}\left(\Omega_{1,>1 / n} \times\left(\widetilde{\Omega}_{2} \backslash \Omega_{2,>1 / n}\right)\right) \stackrel{k \rightarrow \infty}{\longrightarrow} 0 .
$$

We also have that

$$
\left|\nu_{\varepsilon_{k}}\left(F \times \widetilde{\Omega}_{2}\right)-\mu_{1}(F)\right| \leq \varepsilon_{k} \stackrel{k \rightarrow \infty}{\longrightarrow} 0 .
$$

Therefore for any clopen set, $\mu_{1}(F)=\nu_{n}\left(F \times \Omega_{2,>1 / n}\right)$. This implies that $\mu_{1}$ and the projection of $\nu_{n}$ onto $\Omega_{1,>1 / n}$ are the same, which proves (1). 
(2) Since $\Omega_{1,>1 / n} \times \Omega_{2,>1 / n}$ is clopen in $\Omega_{1,>1 /(n+1)} \times \Omega_{2,>1 /(n+1)}$,

$$
\nu_{n+1}\left(\Omega_{1,>1 / n} \times \Omega_{2,>1 / n}\right)=\lim _{k \rightarrow \infty} \nu_{\varepsilon_{k}}\left(\Omega_{1,>1 / n} \times \Omega_{2,>1 / n}\right)=\nu_{n}\left(\Omega_{1,>1 / n} \times \Omega_{2,>1 / n}\right) .
$$

Furthermore, for any closed $F \subseteq \Omega_{1,>1 / n} \times \Omega_{2,>1 / n}, F$ is also closed in $\Omega_{1,1 /(n+1)} \times$ $\Omega_{2,1 /(n+1)}$, which implies that

$$
\limsup _{k \rightarrow \infty} \nu_{\varepsilon_{k}}(F) \leq \nu_{n+1}(F) .
$$

This implies that $\nu_{\varepsilon_{k}}$ converges weakly to $\left.\nu_{n+1}\right|_{\Omega_{1,>1 / n} \times \Omega_{2,>1 / n}}$, but since it also converges to $\nu_{n}$, the two must be equal.

(3) Let $W_{i, n}=\left.\widetilde{W}_{i}\right|_{\left(\Omega_{i,>1 / n}\right)^{2}}$. Since

$$
\left\|\widetilde{W}_{1}^{\pi_{1}}-\widetilde{W}_{2}^{\pi_{2}}\right\|_{2 \rightarrow 2, \nu_{\varepsilon_{k}}} \leq \varepsilon_{k}
$$

we have that in particular, for any $n$,

$$
\left\|W_{1, n}^{\pi_{1}}-W_{2, n}^{\pi_{2}}\right\|_{2 \rightarrow 2, \nu_{\varepsilon_{k}}} \leq \varepsilon_{k} .
$$

This implies that

$$
\left\|W_{1, n}^{\pi_{1}}-W_{2, n}^{\pi_{2}}\right\|_{\square, \nu_{\varepsilon_{k}}} \leq \varepsilon_{k} \mu_{1}\left(\Omega_{1,>1 / n}\right) .
$$

Since $\Omega_{i,>1 / n}$ each have finite measure, we can use Janson's argument in [16]. We present the argument for completeness. Fix $\varepsilon$. We can find step graphons $U_{1, n}$ and $U_{2, n}$ on $\left(\Omega_{1,>1 / n}\right)^{2}$ and $\left(\Omega_{2,>1 / n}\right)^{2}$, with each part in the partition being a clopen set, such that

$$
\left\|U_{i, n}-W_{i, n}\right\|_{1} \leq \varepsilon .
$$

This means that for any coupling measure on $\Omega_{1,>1 / n} \times \Omega_{2,>1 / n}$,

$$
\left\|U_{i, n}^{\pi_{i}}-W_{i, n}^{\pi_{i}}\right\|_{1} \leq \varepsilon
$$

Now, $U_{1, n}^{\pi_{1}}-U_{2, n}^{\pi_{2}}$ is a step function on $\left(\Omega_{1,>1 / n} \times \Omega_{2,>1 / n}\right)^{2}$ with a partition into clopen parts. This means that since the restrictions of $\nu_{\varepsilon_{k}}$ weakly converge to $\nu_{n}$,

$$
\left\|U_{1, n}^{\pi_{1}}-U_{2, n}^{\pi_{2}}\right\|_{\square, \nu_{\varepsilon_{k}}} \stackrel{k \rightarrow \infty}{\longrightarrow}\left\|U_{1, n}^{\pi_{1}}-U_{2, n}^{\pi_{2}}\right\|_{\square, \nu_{n}} .
$$

Take $k$ large enough so that $\varepsilon_{k} \mu_{1}\left(\Omega_{1,>1 / n}\right) \leq \varepsilon$ and

$$
\left|\left\|U_{1, n}^{\pi_{1}}-U_{2, n}^{\pi_{2}}\right\|_{\square, \nu_{\varepsilon_{k}}}-\left\|U_{1, n}^{\pi_{1}}-U_{2, n}^{\pi_{2}}\right\|_{\square, \nu_{n}}\right| \leq \varepsilon
$$

We then have

$$
\begin{aligned}
\| W_{1, n}^{\pi_{1}} & -W_{2, n}^{\pi_{2}} \|_{\square, \nu_{n}} \\
& \leq\left\|W_{1, n}^{\pi_{1}}-U_{1, n}^{\pi_{1}}\right\|_{1, \nu_{n}}+\left\|U_{1, n}^{\pi_{1}}-U_{2, n}^{\pi_{2}}\right\|_{\square, \nu_{n}}+\left\|U_{2, n}^{\pi_{2}}-W_{2, n}^{\pi_{2}}\right\|_{1, \nu_{n}} \\
& \leq 3 \varepsilon+\left\|U_{1, n}^{\pi_{1}}-U_{2, n}^{\pi_{2}}\right\|_{\square, \nu_{\varepsilon_{k}}} \\
& \leq 3 \varepsilon+\left\|W_{1, n}^{\pi_{1}}-U_{1, n}^{\pi_{1}}\right\|_{1, \nu_{\varepsilon_{k}}}+\left\|W_{1, n}^{\pi_{1}}-W_{2, n}^{\pi_{2}}\right\|_{\square, \nu_{\varepsilon_{k}}}+\left\|U_{2, n}^{\pi_{2}}-W_{2, n}^{\pi_{2}}\right\|_{1, \nu_{\varepsilon_{k}}} \\
& \leq 6 \varepsilon .
\end{aligned}
$$

Since this holds for any $\varepsilon$, this proves (3). 
(4) Fix $\varepsilon>0$ and assume that $\varepsilon_{k} \leq \varepsilon$. Since $D_{\widetilde{\mathbb{W}}_{i, \varepsilon_{k}}}(x) \leq D_{\mathbb{W}_{i}}(x) \leq D_{\widetilde{\mathbb{W}}_{i, \varepsilon_{k}}}(x)+\varepsilon_{k}$ for all $x \in \Omega_{i} \cap \widetilde{\Omega}_{i}^{\varepsilon}$,

$$
\begin{aligned}
& \nu_{\varepsilon}\left(\left\{x \in \Omega_{1,>1 / n} \times \Omega_{2,>1 / n}:\left|D_{\mathbb{W}_{1}^{\pi_{1}}}(x)-D_{\mathbb{W}_{2}^{\pi_{2}}}(x)\right| \geq 2 \varepsilon\right\}\right) \\
& \quad \leq \nu_{\varepsilon}\left(\left\{x \in \Omega_{1,>1 / n} \times \Omega_{2,>1 / n}:\left|D_{\widetilde{\mathbb{W}}_{1}^{\pi_{1}, \nu_{\varepsilon}}}(x)-D_{\widetilde{\mathbb{W}}_{2}^{\pi_{2}, \nu_{\varepsilon}}}(x)\right| \geq \varepsilon\right\}\right) \\
& \quad \leq \varepsilon^{-2}\left\|D_{\widetilde{\mathbb{W}}_{1}^{\pi_{1}, \nu_{\varepsilon_{k}}}}-D_{\widetilde{\mathbb{W}}_{2}^{\pi_{2}, \nu_{\varepsilon}}}\right\|_{2}^{2} \leq \varepsilon^{-2} \varepsilon_{k}^{4} .
\end{aligned}
$$

Since for all $\varepsilon>0$ the right side converges to 0 as $k \rightarrow \infty$ this shows that $\nu_{n}$ is supported on $\left\{x \in \Omega_{1,>1 / n} \times \Omega_{2,>1 / n}: D_{\mathbb{W}_{1}^{\pi_{1}}}(x)=D_{\mathbb{W}_{2}^{\pi_{2}}}(x)\right\}$.

Proof of Theorem 8.2. After these preparations, we are ready to define the measure $\mu$ on $\Omega_{1} \times \Omega_{2}$. Note that since before the extensions $D_{\mathbb{W}_{i}}>0$ almost everywhere, we have that $\bigcup_{n} \Omega_{i,>1 / n}=\Omega_{i}$. For $A \subseteq \Omega_{1} \times \Omega_{2}$, let

$$
\begin{aligned}
\nu(A) & =\lim _{n \rightarrow \infty} \nu_{n}\left(A \cap\left(\Omega_{1,>1 / n} \times \Omega_{2,>1 / n}\right)\right) \\
& =\sum_{n=1}^{\infty} \nu_{n}\left(A \cap\left(\left(\Omega_{1,>1 / n} \times \Omega_{2,>1 / n}\right) \backslash\left(\Omega_{1,>1 /(n-1)} \times \Omega_{2,>1 /(n-1)}\right)\right)\right) .
\end{aligned}
$$

Here with a slight abuse of notation we think of $\Omega_{i, 1 / 0}$ as the empty set. To show that this a coupling, note that for any measurable set $X \subseteq \Omega_{1}$,

$$
\begin{aligned}
\mu_{1}(X) & =\lim _{n \rightarrow \infty} \mu_{1}\left(X \cap \Omega_{1,>1 / n}\right) \\
& =\lim _{n \rightarrow \infty} \nu_{n}\left(\left(X \cap \Omega_{1,>1 / n}\right) \times \Omega_{2,>1 / n}\right) \\
& =\lim _{n \rightarrow \infty} \nu_{n}\left(\left(X \times \Omega_{2}\right) \cap\left(\Omega_{1,>1 / n} \times \Omega_{2,>1 / n}\right)\right)=\nu\left(X \times \Omega_{2}\right),
\end{aligned}
$$

where in the second step we used that for each $n$, the restriction of $\nu$ to $\Omega_{1,>1 / n} \times$ $\Omega_{2,>1 / n}$ is equal to $\nu_{n}$, which is a coupling. Clearly the analogous argument works for subsets $Y \subseteq \Omega_{2}$.

Now, let

$$
N=\left\{(x, y)=\left(\left(x_{1}, x_{2}\right),\left(y_{1}, y_{2}\right)\right) \in\left(\Omega_{1} \times \Omega_{2}\right)^{2}: W_{1}^{\pi_{1}}(x, y) \neq W_{2}^{\pi_{2}}(x, y)\right\}
$$

and

$$
M=\left\{x=\left(x_{1}, x_{2}\right) \in\left(\Omega_{1} \times \Omega_{2}\right): D_{\mathbb{W}_{1}^{\pi_{1}}}(x) \neq D_{\mathbb{W}_{2}^{\pi_{2}}}(x)\right\}
$$

Let

$$
N_{n}=\left\{(x, y) \in\left(\Omega_{1,>1 / n} \times \Omega_{2,>1 / n}\right)^{2}: W_{1}^{\pi_{1}}(x, y) \neq W_{2}^{\pi_{2}}(x, y)\right\}
$$

and

$$
M_{n}=\left\{x=\left(x_{1}, x_{2}\right) \in\left(\Omega_{1,>1 / n} \times \Omega_{2,>1 / n}\right): D_{\mathbb{W}_{1}^{\pi_{1}}}(x) \neq D_{\mathbb{W}_{2}^{\pi_{2}}}(x)\right\} .
$$

Since $\bigcup_{n} \Omega_{i,>1 / n}=\Omega_{i}$ and $\Omega_{i,>1 / n} \subseteq \Omega_{i, 1 /(n+1)}$, we have that $N=\bigcup_{n} N_{n}$ and $M=\bigcup_{n} M_{n}$. By Lemma $8.6(3,4),(\nu \times \nu)\left(N_{n}\right)=\left(\nu_{n} \times \nu_{n}\right)\left(N_{n}\right)=0$ and $\nu\left(M_{n}\right)=0$, which implies that $(\nu \times \nu)(N)=0$ and $\nu(M)=0$, and hence $W_{1}^{\pi_{1}}=W_{2}^{\pi_{2}}$ and $D_{\mathbb{W}_{1}^{\pi_{1}}}=D_{\mathbb{W}_{2}^{\pi_{2}}}$ almost everywhere. Since $W_{1}^{\pi_{1}}=W_{2}^{\pi_{2}}$ almost everywhere implies that $D_{W_{1}^{\pi_{1}}}=D_{W_{2}^{\pi_{2}}}$ almost everywhere, this in turn implies that $S_{1}^{\pi_{1}}=S_{2}^{\pi_{2}} \nu$-almost everywhere.

To prove that $I_{1}=I_{2}$, we again use Proposition 4.7, but instead of (3) we this time use $(2)$. Fix $D>0$. Since $\delta_{2 \rightarrow 2}\left(\mathbb{W}_{1, \leq D}, \mathbb{W}_{2, \leq D}\right)=0$, we in particular have 
that $\left\|\mathbb{W}_{1, \leq D}\right\|_{1}=\left\|\mathbb{W}_{2, \leq D}\right\|_{1}$. But since $W_{1}^{\pi_{1}}=W_{2}^{\pi_{2}}$ and $D_{\mathbb{W}_{1}^{\pi_{1}}}=D_{\mathbb{W}_{2}^{\pi_{2}}}$ almost everywhere,

$$
\begin{aligned}
\int_{\left(\Omega_{1}, \leq D\right)^{2}} W_{1} d \mu_{1} \times d \mu_{1} & =\int_{\left(\Omega_{1} \times \Omega_{2}\right)^{2}} W_{1}^{\pi_{1}} 1_{D_{\mathrm{w}_{1}} \pi_{1} \leq D} d \nu \times d \nu \\
& =\int_{\left(\Omega_{1} \times \Omega_{2}\right)^{2}} W_{2}^{\pi_{2}} 1_{D_{\mathrm{w}_{2}} \leq D} d \nu \times d \nu \\
& =\int_{\left(\Omega_{2}, \leq D\right)^{2}} W_{2} d \mu_{2} \times d \mu_{1} .
\end{aligned}
$$

In a similar way, $\int_{\Omega_{1}, \leq D} S_{1}=\int_{\Omega_{2}, \leq D} S_{2}$. Therefore $\left\|\mathbb{W}_{1, \leq D}\right\|_{1}=\left\|\mathbb{W}_{2, \leq D}\right\|_{1}$ implies $I_{1}=I_{2}$.

Corollary 8.7. Let $\mathbb{W}=(W, S, I, \boldsymbol{\Omega})$ and $\mathbb{W}^{\prime}=\left(W^{\prime}, S^{\prime}, I^{\prime}, \boldsymbol{\Omega}^{\prime}\right)$ be graphexes that are equivalent, and suppose that $D_{\mathbb{W}}, D_{\mathbb{W}^{\prime}}>0$ everywhere. Then there exist a positive integer $n$ and a chain of graphexes $\mathbb{W}_{i}=\left(W_{i}, S_{i}, I_{i}, \boldsymbol{\Omega}_{i}\right)$ for $i=0, \ldots, n$, with $D_{\mathbb{W}_{i}}>0$ everywhere for each $i=0, \ldots, n, \mathbb{W}_{0}=\mathbb{W}, \mathbb{W}_{n}=\mathbb{W}^{\prime}$, and for each $i \geq 1$, either $\mathbb{W}_{i-1}=\mathbb{W}_{i}^{\phi_{i}}$ almost everywhere for some measure preserving map $\phi_{i}$ from $\boldsymbol{\Omega}_{i-1}$ to $\boldsymbol{\Omega}_{i}$, or $\mathbb{W}_{i}=\mathbb{W}_{i-1}^{\phi_{i}}$ almost everywhere for some $\phi_{i}$ from $\boldsymbol{\Omega}_{i}$ to $\boldsymbol{\Omega}_{i-1}$. In fact, we can take $n=4$.

Proof. By the construction in the next section (which itself does not use Corollary 8.7), there exists $\mathbb{W}_{1}=\left(W_{1}, S_{1}, I_{1}, \boldsymbol{\Omega}_{1}\right)$ such that $\boldsymbol{\Omega}_{1}$ is Borel and a measurepreserving map $\phi_{1}$ from $\boldsymbol{\Omega}_{0}$ to $\boldsymbol{\Omega}_{1}$ with $W=W_{0}=W_{1}^{\phi_{1}}$ almost everywhere. Then $\Omega_{0}=\operatorname{dsupp} W_{0}=\phi^{-1}\left(\operatorname{dsupp} W_{1}\right)$, so by replacing $\boldsymbol{\Omega}_{1}$ by its restriction to dsupp $W_{2}$, we may assume that $D_{W_{1}}>0$ everywhere. Similarly, there exists $\left(W_{3}, \boldsymbol{\Omega}_{3}\right)$ with $\Omega_{3}$ Borel and a measure preserving map $\phi_{4}$ from $\boldsymbol{\Omega}_{4}$ to $\boldsymbol{\Omega}_{3}$ with $W^{\prime}=W_{4}=W_{3}^{\phi_{4}}$ almost everywhere and $D_{W_{3}}>0$ everywhere. Now, we can apply Theorem 7.1 to show that $\delta_{\diamond}\left(\mathbb{W}_{1}, \mathbb{W}_{3}\right)=0$. We can then apply Theorem 8.2 to $\left(\mathbb{W}_{1}, \boldsymbol{\Omega}_{1}\right)$ and $\left(\mathbb{W}_{3}, \boldsymbol{\Omega}_{3}\right)$ to find a Borel space $\boldsymbol{\Omega}_{2}$ and measure preserving maps $\phi_{2}$ from $\boldsymbol{\Omega}_{2}$ to $\boldsymbol{\Omega}_{1}$ and $\phi_{3}$ from $\boldsymbol{\Omega}_{2}$ to $\boldsymbol{\Omega}_{3}$ such that $W_{3}^{\phi_{3}}=W_{1}^{\phi_{2}}$ almost everywhere. We can then take $W_{2}$ to be, say, $W_{1}^{\phi_{2}}$.

8.2. Canonical graphex. In this section, we prove Theorem 8.1. We follow the approach of Janson in [16], based on the construction of Lovász and Szegedy in [23].

Concretely, given a graphex $\mathbb{W}=(W, S, I, \boldsymbol{\Omega})$, where $\boldsymbol{\Omega}=(\Omega, \mathcal{F}, \mu)$, define a map $\psi_{W}: \Omega \rightarrow L^{1}(\Omega, \mathcal{F}, \mu)$ by $x \mapsto W(x, \cdot)$, and define a map $\psi_{\mathbb{W}}: \Omega \rightarrow L^{1}(\Omega, \mathcal{F}, \mu) \times \mathbb{R}$ by $x \mapsto(W(x, \cdot), S(x))$, where we equip $L^{1}(\Omega, \mathcal{F}, \mu)$ with the standard Borel $\sigma$ algebra and $L^{1}(\Omega, \mathcal{F}, \mu) \times \mathbb{R}$ with the standard product Borel $\sigma$-algebra. Note that in general, we only know that $\psi_{W}(x) \in L^{1}(\Omega, \mathcal{F}, \mu)$ for almost all $x \in \Omega$, but by changing $W$ on a set of measure zero, we may assume that this holds for all $x \in \Omega$; we will assume that throughout this section. We will see in Lemma 8.9 that $\psi_{W}$, and thus $\psi_{\mathbb{W}}$, is measurable. Defining $\mu_{W}$ and $\mu_{\mathbb{W}}$ as the pushforward of $\mu$ under $\psi_{W}$ and $\psi_{\mathbb{W}}$ respectively, and $\Omega_{W}$ and $\Omega_{\mathbb{W}}$ as the corresponding supports, we then construct a graphex $\widehat{\mathbb{W}}$ over $\Omega_{\mathbb{W}}$ (equipped with the Borel $\sigma$-algebra and the measure $\left.\mu_{\mathbb{W}}\right)$ such that $\mathbb{W}$ is almost everywhere equal to a pullback of $\widehat{\mathbb{W}}$. Furthermore, we will show that if $\mathbb{W}^{\prime}$ is a.e. equal to a pullback of $\mathbb{W}$, then we can find a measure preserving bijection $\phi^{*}$ from $\Omega_{\mathbb{W}}$ to $\Omega_{\mathbb{W}}^{\prime}$ such that $\widehat{\mathbb{W}}=\left(\widehat{\mathbb{W}^{\prime}}\right)^{\phi^{*}}$ a.e. Combined with Corollary 8.7, this will establish Theorem 8.1. 
We first state some preliminary lemmas. Recall that if $(\Omega, \mathcal{F}, \mu)$ is a measure space, then $L^{1}(\Omega, \mathcal{F}, \mu)$ is a Banach space where each point is an equivalence class consisting of integrable measurable functions from $\Omega$ to $\mathbb{R}$, where two functions are equivalent if they are equal almost everywhere (or equivalently their $L^{1}$ distance is 0$)$. Note that in general, the space $L^{1}(\Omega, \mathcal{F}, \mu)$ is not separable, a fact which will lead to technical complications when considering measurable functions into $L^{1}(\Omega, \mathcal{F}, \mu)$ (e.g., the sum of two such functions is in general not measurable). As in [16], we will avoid these difficulties by carefully constructing separable subspaces of $L^{1}(\Omega, \mathcal{F}, \mu)$ such that the functions of interest take values in these subspaces. See Lemma 8.8 below.

Throughout this section, we will frequently consider two measure spaces $(\Omega, \mathcal{F}, \mu)$ and $\left(\Omega^{\prime}, \mathcal{F}^{\prime}, \mu^{\prime}\right)$, where $\Omega=\Omega^{\prime}, \mathcal{F}^{\prime} \subseteq \mathcal{F}$, and $\mu^{\prime}$ is the restriction of $\mu$ to $\mathcal{F}^{\prime}$. With a slight abuse of notation, we will often denote the second space by $\left(\Omega, \mathcal{F}^{\prime}, \mu\right)$, rather than $\left(\Omega, \mathcal{F}^{\prime},\left.\mu\right|_{\mathcal{F}^{\prime}}\right)$.

As already noted, the space $L^{1}(\Omega, \mathcal{F}, \mu)$ is in general not separable (if $\Omega$ is a Borel space, it is, but we want to define this construction for general $\Omega$ ). We will therefore need the following lemma.

Lemma 8.8. Suppose that $(\Omega, \mathcal{F}, \mu)$ and $\left(\Omega^{\prime}, \mathcal{F}^{\prime}, \mu^{\prime}\right)$ are $\sigma$-finite measure spaces, $W: \Omega \times \Omega^{\prime} \rightarrow \mathbb{R}$ is measurable, and for all $x \in \Omega$, the function $W(x, \cdot)$ is integrable. Let $\psi_{W}: \Omega \rightarrow L^{1}(\Omega, \mathcal{F}, \mu)$ be the map $x \mapsto W(x, \cdot)$. Then we can find a separable closed subspace $B$ of $L^{1}\left(\Omega^{\prime}, \mathcal{F}^{\prime}, \mu^{\prime}\right)$ such that $\psi_{W}(x) \in B$ for all $x \in \Omega$.

Proof. First, assume that $W$ is bounded and both $\mu(\Omega)<\infty$ and $\mu\left(\Omega^{\prime}\right)<\infty$. The statement of the lemma then clearly holds for all step functions, and by a monotone class argument it holds for all bounded $W$.

Next, relax the condition that $\mu^{\prime}\left(\Omega^{\prime}\right)<\infty$. Let $\Omega_{1}^{\prime} \subseteq \Omega_{2}^{\prime} \subseteq \cdots \subseteq \Omega_{n}^{\prime} \subseteq \ldots$ be a sequence of measurable subsets of $\Omega^{\prime}$ with finite measure and $\bigcup_{n=1}^{\infty} \Omega_{n}^{\prime}=\Omega^{\prime}$. Then for every $n$ we can find a separable closed subspace $B_{n} \subseteq L^{1}\left(\Omega_{n}^{\prime},\left.\mathcal{F}^{\prime}\right|_{\Omega_{n}^{\prime}},\left.\mu^{\prime}\right|_{\Omega_{n}^{\prime}}\right)$ such that for every $x \in \Omega,\left.W(x, \cdot)\right|_{\Omega_{n}^{\prime}} \in B_{n}$. Let $\widetilde{B}_{n}$ consist of those $f \in L^{1}\left(\Omega^{\prime}, \mathcal{F}^{\prime}, \mu^{\prime}\right)$ that have $\left.f\right|_{\Omega_{n}^{\prime}} \in B_{n}$ and $\left.f\right|_{\Omega^{\prime}-\Omega_{n}^{\prime}} \equiv 0$. Clearly $\widetilde{B}_{n}$ is isomorphic to $B_{n}$, and thus separable. Let $B$ be the closure of the space generated by $\bigcup_{n} \widetilde{B}_{n}$; this is separable. We claim that for any $x \in \Omega$, the function $W(x, \cdot)$ is contained in $B$. It suffices to show that for any $\varepsilon$, there is an $n \in \mathbb{N}$ and a $g \in \widetilde{B}_{n}$ such that $\|W(x, \cdot)-g\|_{1}<\varepsilon$. Since $W(x, \cdot) \in L^{1}\left(\Omega^{\prime}, \mathcal{F}^{\prime}, \mu^{\prime}\right)$, we can take $n$ large enough that $\left\|W(x, \cdot)-W(x, \cdot) \chi\left(\Omega_{n}^{\prime}\right)\right\|_{1}<\varepsilon$. But then by the definition of $\widetilde{B}_{n}$, we have $W(x, \cdot) \chi\left(\Omega_{n}^{\prime}\right) \in \widetilde{B}_{n}$, so taking $g=W(x, \cdot) \chi\left(\Omega_{n}^{\prime}\right)$, we are done.

In a similar way, we can approximate an unbounded $W$ by the function $W 1_{|W| \leq n}$ to relax the condition that $W$ is bounded.

Finally, for general $\Omega$ and $\Omega^{\prime}$, we can write $\Omega$ as the disjoint union of finite measure sets $\Omega_{1}, \Omega_{2}, \ldots, \Omega_{n}, \ldots$. We know that the image of each $\Omega_{n}$ is contained in a separable closed subspace $B_{n} \subseteq L^{1}\left(\Omega^{\prime}, \mathcal{F}^{\prime}\right)$. Therefore, taking $B$ to be the closure of the subspace generated by $\bigcup_{n} B_{n}$, the image of the map $\psi_{W}$ is contained in $B$.

Lemma 8.9. Suppose $(\Omega, \mathcal{F}, \mu)$ and $\left(\Omega^{\prime}, \mathcal{F}^{\prime}, \mu^{\prime}\right)$ are $\sigma$-finite measure spaces, $W: \Omega \times$ $\Omega^{\prime} \rightarrow \mathbb{R}$ is $\mathcal{F} \times \mathcal{F}^{\prime}$-measurable, and that for all $x \in \Omega$, the function $W(x, \cdot)$ is integrable. Then the map $\psi_{W}: \Omega \rightarrow L^{1}\left(\Omega^{\prime}, \mathcal{F}^{\prime}, \mu^{\prime}\right)$ with $x \mapsto W(x, \cdot)$ is measurable with respect to the standard Borel $\sigma$-algebra on $L^{1}(\Omega, \mathcal{F}, \mu)$. . 
Proof. Our goals is to show that $c \in[0, \infty)$ and any $f \in L^{1}\left(\Omega^{\prime}, \mathcal{F}^{\prime}, \mu^{\prime}\right)$, the set

$$
B(f, W, c)=\left\{x \in \Omega:\|W(x, \cdot)-f\|_{1} \leq c\right\}
$$

is measurable.

Assume that $x \in B(f, W, c)$, and let $F_{W} \subset L^{1}\left(\Omega^{\prime}, \mathcal{F}^{\prime}, \mu^{\prime}\right)$ be a countable set such that $W(x, \cdot)$ lies in the closure of $F_{W}$ for all $x \in \Omega$ (the existence of such a set follows from Lemma 8.8). Given any $\varepsilon>0$, we can then find an $\widehat{f} \in F_{W}$ such that $x \in B(\widehat{f}, W, \varepsilon)$, and $\|f-\widehat{f}\|_{1} \leq c+\varepsilon$. If, on the other hand, $\|f-\widehat{f}\|_{1} \leq c+\varepsilon$ and $x \in B(\widehat{f}, W, \varepsilon)$ then $x \in B(f, W, c+2 \varepsilon)$. Since $B(f, W, c)=\bigcap_{i} B\left(f, W, c+\varepsilon_{i}\right)$ whenever $\varepsilon_{i} \rightarrow 0$, this proves that it is enough to prove measurability of $B\left(\widehat{f}, W, \varepsilon_{i}\right)$ for all $\widehat{f} \in F_{W}$ and an arbitrary sequence $\varepsilon_{i} \in(0, \infty)$ such that $\varepsilon_{i} \rightarrow 0$.

Using this observation, it is easy to see that if $W_{1}$ and $W_{2}$ obey the conclusions of the lemma, then so does any linear combination. The lemma is clearly also true for all step functions. A standard monotone class argument then implies that the lemma holds for all bounded, measurable $W$.

If $W$ is unbounded, we use that by assumption, $\psi_{W}(x) \in L^{1}\left(\Omega^{\prime}, \mathcal{F}^{\prime}, \mu^{\prime}\right)$ for all for all $x \in \Omega$. Using this fact, one easily shows that

$$
B(f, W, c)=\bigcap_{n=1}^{\infty} B\left(f, W(x, \cdot) 1_{|W(x, \cdot)| \leq n}, c\right) .
$$

Since $B\left(f, W(x, \cdot) 1_{|W(x, \cdot)| \leq n}, c\right)$ is measurable, this proves the statement for unbounded $W$,

We will also use the following technical lemma, which is Lemma G.1 in [16] (the proof also works for $\sigma$-finite measures):

Lemma 8.10. Let $(\Omega, \mathcal{F}, \mu)$ be any $\sigma$-finite measure space, and $B \subseteq L^{1}(\Omega, \mathcal{F}, \mu)$ a closed separable subspace. Then there exists a measurable evaluation map

$$
\Phi: B \times \Omega \rightarrow \mathbb{R}
$$

such that for any $f \in B$, for almost every $x \in \Omega, f(x)=\Phi(f, x)$ (note that $f(x)$ is only defined almost everywhere). In particular, if $L^{1}(\Omega, \mathcal{F}, \mu)$ is separable, we can take $B=L^{1}(\Omega, \mathcal{F}, \mu)$.

We need one more lemma.

Lemma 8.11. Suppose that $(\Omega, \mathcal{F}, \mu)$ and $\left(\Omega^{\prime}, \mathcal{F}^{\prime}, \mu^{\prime}\right)$ are $\sigma$-finite measure spaces, $W: \Omega \times \Omega^{\prime} \rightarrow \mathbb{R}$ is measurable, and for all $x \in \Omega$ and $x^{\prime} \in \Omega^{\prime}$, both $W(x, \cdot)$ and $W\left(\cdot, x^{\prime}\right)$ are integrable. Let $\psi_{W}$ be the map $\Omega \rightarrow L^{1}\left(\Omega^{\prime}, \mathcal{F}^{\prime}, \mu^{\prime}\right)$ with $x \mapsto W(x, \cdot)$, and $\psi_{W}^{\prime}$ be the map $\Omega^{\prime} \rightarrow L^{1}(\Omega, \mathcal{F}, \mu)$ with $y \mapsto W(\cdot, y)$. Further, let $\mathcal{G}$ be the Borel $\sigma$-algebra on $L^{1}(\Omega, \mathcal{F}, \mu)$, and let

$$
\mathcal{F}_{W}^{\prime}=\psi_{W}^{\prime-1}(\mathcal{G})
$$

Then for almost every $x \in \Omega, \psi_{W}(x) \in L^{1}\left(\Omega^{\prime}, \mathcal{F}_{W}^{\prime}, \mu^{\prime}\right)$.

Proof. By Lemma 8.8, the image of $\psi_{W}^{\prime}$ is contained in a separable subspace $B \subseteq L^{1}(\Omega, \mathcal{F}, \mu)$. By Lemma 8.10, there exists a measurable map $\Phi: B \times \Omega \rightarrow \mathbb{R}$ such that for any $f \in B$ and almost any $x \in \Omega, f(x)=\Phi(f, x)$. Define $\widetilde{W}$ on $\Omega \times \Omega^{\prime}$ by

$$
\widetilde{W}(x, y)=\Phi\left(\psi_{W}^{\prime}(y), x\right) .
$$


Then for every $y$ and almost every $x, \widetilde{W}(x, y)=W(x, y)$, so $\widetilde{W}=W$ almost everywhere on $\Omega \times \Omega^{\prime}$. Thus, if we define $\psi_{\widetilde{W}}$ analogously to $\psi_{W}$, then for almost every $x \in \Omega,\left\|\psi_{\widetilde{W}}(x)-\psi_{W}(x)\right\|_{L^{1}\left(\Omega^{\prime}, \mathcal{F}^{\prime}, \mu^{\prime}\right)}=0$; that is, for almost all $x, \psi_{W}(x)=\psi_{\widetilde{W}}(x)$ as elements of $L^{1}\left(\Omega^{\prime}, \mathcal{F}^{\prime}, \mu^{\prime}\right)$. However, since $\Phi$ is $B \times \Omega$-measurable, and $\psi_{W}^{\prime}: \Omega^{\prime} \rightarrow B$ is measurable, $\widetilde{W}$ is $\mathcal{F} \times \mathcal{F}_{W}^{\prime}$-measurable, so in particular $\psi_{\widetilde{W}}(x)$ is $\mathcal{F}_{W}^{\prime}$-measurable for all $x$. Since $\psi_{W}(x)=\psi_{\widetilde{W}}(x) \in L^{1}\left(\Omega^{\prime}, \mathcal{F}^{\prime}, \mu^{\prime}\right)$ for almost every $x$, it follows that $\psi_{W}(x) \in L^{1}\left(\Omega^{\prime}, \mathcal{F}_{W}^{\prime}, \mu^{\prime}\right)$ for almost every $x \in \Omega$.

Let $(W, S, I, \boldsymbol{\Omega})$ be a graphex over $\boldsymbol{\Omega}=(\Omega, \mathcal{F}, \mu)$ such that $W(x, \cdot)$ is integrable for all $x$. For each $x \in \Omega$, we have the section $W_{x} \in L^{1}(\Omega, \mathcal{F}, \mu)$ defined by $W_{x}(y)=W(x, y)$, giving us the map

$$
\psi_{W}: \Omega \rightarrow L^{1}(\Omega, \mathcal{F}, \mu)
$$

defined by $x \mapsto W_{x}$. Let

$$
\psi_{\mathbb{W}}: \Omega \rightarrow L^{1}(\Omega, \mathcal{F}, \mu) \times \mathbb{R}
$$

be defined by $x \mapsto\left(W_{x}, S(x)\right)$. By Lemma $8.9, \psi_{W}$, and thus $\psi_{\mathbb{W}}$, is measurable. Let $\mu_{W}=\mu^{\psi_{W}}$ and $\mu_{\mathbb{W}}=\mu^{\psi_{\mathbb{W}}}$, and let $\Omega_{W} \subseteq L^{1}(\Omega, \mathcal{F}, \mu)$ and $\Omega_{\mathbb{W}} \subseteq L^{1}(\Omega, \mathcal{F}, \mu) \times \mathbb{R}$ be the supports of $\mu_{W}$ and $\mu_{\mathbb{W}}$, respectively, i.e.,

$\Omega_{W}=\left\{f \in L^{1}(\Omega, \mathcal{F}, \mu): \mu_{W}(U)>0\right.$ for every open $U \subseteq L^{1}(\Omega, \mathcal{F}, \mu)$ with $\left.f \in U\right\}$, and

$$
\begin{aligned}
& \Omega_{\mathbb{W}}=\left\{(f, c) \in L^{1}(\Omega, \mathcal{F}, \mu) \times \mathbb{R}:\right. \\
& \left.\quad \mu_{\mathbb{W}}(U)>0 \text { for every open } U \subseteq L^{1}(\Omega, \mathcal{F}, \mu) \times \mathbb{R} \text { with }(f, c) \in U\right\} .
\end{aligned}
$$

Alternatively, we can also define $\psi_{\mathbb{W}}$ and $\mu_{\mathbb{W}}$ as follows. Let $(\widetilde{\Omega}, \widetilde{\mathcal{F}}, \widetilde{\mu})$ be defined as $\widetilde{\Omega}=\Omega \cup\left\{\Omega_{\infty}\right\}$, where $\Omega_{\infty}$ is an atom with measure 1 . Then we can think of $\psi_{\mathbb{W}}(x)$ as a function in $L^{1}(\widetilde{\Omega}, \widetilde{\mathcal{F}}, \widetilde{\mu})$, with $\psi_{\mathbb{W}}(x)(y)=W(x, y)$ if $y \in \Omega$ and $\psi_{\mathbb{W}}(x)(y)=S(x)$ if $y=\Omega_{\infty}$, and $\left\|\psi_{\mathbb{W}}(x)\right\|_{1}=D_{\mathbb{W}}(x)$. This gives a bijection $L^{1}(\widetilde{\Omega}, \widetilde{\mathcal{F}}, \widetilde{\mu}) \equiv L^{1}(\Omega, \mathcal{F}, \mu) \times \mathbb{R}$ and $\psi_{\mathbb{W}}$ as a map from $\Omega$ to $L^{1}(\widetilde{\Omega}, \widetilde{\mathcal{F}}, \widetilde{\mu})$. Note that $\mu_{W}$ is the projection of $\mu_{\mathbb{W}}$, and thus $\Omega_{W}$ is the closure of the projection of $\Omega_{\mathbb{W}}$. Equipping $\Omega_{W}$ with the standard Borel $\sigma$-algebra $\mathcal{G}_{W}$, this gives us a measure space $\left(\Omega_{W}, \mathcal{G}_{W}, \mu_{W}\right)$, and similarly we obtain $\Omega_{\mathbb{W}}=\left(\Omega_{\mathbb{W}}, \mathcal{G}_{\mathbb{W}}, \mu_{\mathbb{W}}\right)$.

Let $\mathcal{G}$ and $\widetilde{\mathcal{G}}$ be the Borel $\sigma$-algebra on $L^{1}(\Omega, \mathcal{F}, \mu)$ and $L^{1}(\widetilde{\Omega}, \widetilde{\mathcal{F}}, \widetilde{\mu})$, respectively. Via the maps $\psi_{W}$ and $\psi_{\mathbb{W}}$ they induce two different $\sigma$-algebras on $\Omega$, the $\sigma$-algebras

$$
\mathcal{F}_{W}=\psi_{W}^{-1}(\mathcal{G})
$$

and

$$
\mathcal{F}_{\mathbb{W}}=\psi_{\mathbb{W}}^{-1}(\widetilde{\mathcal{G}})
$$

We also define $\widetilde{\mathcal{F}}_{W}=\mathcal{F}_{W} \times \mathcal{B}$ and $\widetilde{\mathcal{F}}_{\mathbb{W}}=\mathcal{F}_{\mathbb{W}} \times \mathcal{B}$. Note that $\mathcal{F}_{W} \subseteq \mathcal{F}_{\mathbb{W}}$, with the example of a zero graphon but a nonconstant $S$ function showing that strict inequality is possible.

It is easy to see that if $\mathbb{W}^{\prime}$ is equal to $\mathbb{W}$ almost everywhere, then $\mu_{\mathbb{W}}=\mu_{\mathbb{W}}$ and hence $\Omega_{\mathbb{W}}=\Omega_{\mathbb{W}^{\prime}}$. Indeed, if $\mathbb{W}=\mathbb{W}^{\prime}$ a.e., then for almost all $x, \psi_{\mathbb{W}}(x)=\psi_{\mathbb{W}^{\prime}}(x)$ when viewed as vectors in $L^{1}$. This implies that there exists a set $N \subseteq \Omega$ of measure zero such that for all $A \in \widetilde{\mathcal{G}}$, the symmetric difference of $\psi_{\mathbb{W}}^{-1}(A)$ and $\psi_{\mathbb{W}^{\prime}}^{-1}(A)$ lies in $N$, which shows that $\mu_{\mathbb{W}}=\mu_{\mathbb{W}}$. Furthermore, under the same change, $\mathcal{F}_{W}, \mathcal{F}_{\mathbb{W}}$ and $\mathcal{F}_{W^{\prime}}, \mathcal{F}_{\mathbb{W}^{\prime}}$ only change on a set of measure zero, implying that the Banach spaces $L^{1}\left(\Omega, \mathcal{F}_{W}, \mu\right)$ and $L^{1}\left(\Omega, \mathcal{F}_{\mathbb{W}}, \mu\right)$ remain unchanged. 
Note that in general, $\mu_{\mathbb{W}}$ is not $\sigma$-finite. Indeed, choosing $W$ to be the graphon 0 over any space of infinite measure, and $I, S$ to be 0 , we have that $\psi_{\mathbb{W}}^{-1}(A)=\Omega$ for every $A$ containing the origin $(0) \in L^{1}(\widetilde{\Omega}, \widetilde{\mathcal{F}}, \widetilde{\mu})$, so $\mu_{\mathbb{W}}(A)=\mu(\Omega)=\infty$ if $0 \in A$, and $\mu_{\mathbb{W}}(A)=0$ otherwise. So in particular $\Omega_{\mathbb{W}}=\{(0)\}$ and $\mu_{\mathbb{W}}\left(\Omega_{\mathbb{W}}\right)=\infty$. This also means $\mu_{W}$ is not $\sigma$-finite.

It turns out, however, that this problem can be avoided if we require that the set where $D_{\mathbb{W}}=0$ has measure zero; see Lemma 8.13 below. Before stating the lemma, we prove the following:

Proposition 8.12. The space $\Omega_{\mathbb{W}}$ as defined above is a complete, separable metric space, with the metric induced by $L^{1}(\widetilde{\Omega}, \widetilde{\mathcal{F}}, \widetilde{\mu})$, and $\mu_{\mathbb{W}}$ has full support in $\Omega_{\mathbb{W}}$. Furthermore, $\Omega_{\mathbb{W}} \subseteq L^{1}\left(\widetilde{\Omega}, \widetilde{\mathcal{F}}_{W}, \widetilde{\mu}\right) \subseteq L^{1}\left(\widetilde{\Omega}, \widetilde{\mathcal{F}}_{\mathbb{W}}, \widetilde{\mu}\right)$. Finally, after modifying $W$ and $S$ on a set of measure zero $\psi_{\mathbb{W}}$ becomes an everywhere defined, measure-preserving map from $(\Omega, \mathcal{F}, \mu)$ to $\left(\Omega_{\mathbb{W}}, \mathcal{G}_{\mathbb{W}}, \mu_{\mathbb{W}}\right)$, and we furthermore have $\psi_{\mathbb{W}}^{-1}\left(\mathcal{G}_{\mathbb{W}}\right)=\mathcal{F}_{\mathbb{W}}$.

Proof. By Lemma 8.8, the image of $\psi_{W}$ is contained in a closed separable subspace $B$ of $L^{1}(\Omega, \mathcal{F}, \mu)$. This means that the image of $\psi_{\mathbb{W}}$ is contained in $\widetilde{B}=B \times \mathbb{R}$, which is a closed separable subspace of $L^{1}(\widetilde{\Omega}, \widetilde{\mathcal{F}}, \widetilde{\mu})$. We will show that in fact

$$
\Omega_{W}=\left\{f \in B: \mu_{W}(U)>0 \text { for every open } U \subseteq B \text { with } f \in U\right\} .
$$

and

$$
\Omega_{\mathbb{W}}=\left\{(f, c) \in \widetilde{B}: \mu_{\mathbb{W}}(U)>0 \text { for every open } U \subseteq \widetilde{B} \text { with }(f, c) \in U\right\} .
$$

Indeed, since $\psi_{W}^{-1}\left(L^{1}(\Omega, \mathcal{F}, \mu) \backslash B\right)=\emptyset$, we have $\mu_{W}\left(L^{1}(\Omega, \mathcal{F}, \mu) \backslash B\right)=0$, which in turn implies that support of $\mu_{W}$ is contained in $B$. Let, for a moment, the above defined set be $\Omega_{W}^{\prime}$. First, if $f \in \Omega_{W}^{\prime}$, then for any open set $U \subseteq L^{1}(\Omega, \mathcal{F}, \mu)$ with $f \in U$, we have $\mu_{W}(U) \geq \mu_{W}(U \cap B)>0$. Conversely, if $f \in \Omega_{W}$, we know we must have $f \in B$, and for any open $U \subseteq B$ with $f \in U$, we can find an open $V \subseteq L^{1}(\Omega, \mathcal{F}, \mu)$ with $U=V \cap B$, so in particular $f \in V$. Then $\mu_{W}(V)>0$, and since $\mu_{W}\left(L^{1}(\Omega, \mathcal{F}, \mu) \backslash B\right)=0$, we have $\mu_{W}(U)=\mu_{W}(V)>0$, showing that $f \in \Omega_{W}^{\prime}$. A similar, argument shows the second claim, noting that $\Omega_{\mathbb{W}} \subseteq \widetilde{B}=B \times \mathbb{R}$.

Now take the union $V$ of all open sets $U \subseteq \widetilde{B}$ with $\mu_{\mathbb{W}}(U)=0$. Then $\Omega_{\mathbb{W}}=\widetilde{B} \backslash V$. Since $\widetilde{B}$ is separable and thus second countable, we can find a countable collection $U_{1}, U_{2}, \ldots, U_{n}, \ldots$ with $\mu_{\mathbb{W}}\left(U_{n}\right)=0$ and $\bigcup_{n} U_{n}=V$. This means that $\mu_{\mathbb{W}}(V)=0$, so $\mu\left(\psi_{\mathbb{W}}^{-1}(V)\right)=0$, and thus almost every point in $\Omega$ is mapped to $\Omega_{\mathbb{W}}$. Since $V$ is open, $\Omega_{\mathbb{W}}$ is closed in $\widetilde{B}$, so it is a closed subset of a separable Banach space; thus it is a complete, separable metric space.

To see that $\mu_{\mathbb{W}}$ has full support, consider an open subset $U \subseteq \Omega_{\mathbb{W}}$ with $\mu_{\mathbb{W}}(U)=0$. Since $\Omega_{\mathbb{W}}$ is closed in $\widetilde{B}$ and $\mu_{\mathbb{W}}\left(\widetilde{B} \backslash \Omega_{\mathbb{W}}\right)=0$, we can find an open subset $\tilde{U} \subseteq \widetilde{B}$ such that $U=\tilde{U} \cap \Omega_{\mathbb{W}}$ and $\mu_{\mathbb{W}}(\tilde{U})=\mu_{\mathbb{W}}(U)=0$, so in particular $\tilde{U} \subseteq V=\widetilde{B} \backslash \Omega_{\mathbb{W}}$. But this implies $U=\tilde{U} \cap \Omega_{\mathbb{W}}=\emptyset$, as required.

Next, we use Lemma 8.11 to infer that $\psi_{W}(x) \in L^{1}\left(\Omega, \mathcal{F}_{W}, \mu\right)$ for almost all $x \in \Omega$, which in turn implies that $\psi_{\mathbb{W}}(x) \in L^{1}\left(\widetilde{\Omega}, \widetilde{\mathcal{F}}_{W}, \widetilde{\mu}\right)$ for almost all $x \in \Omega$. As a consequence, the open set $U=L^{1}(\widetilde{\Omega}, \widetilde{\mathcal{F}}, \widetilde{\mu}) \backslash L^{1}\left(\widetilde{\Omega}, \widetilde{\mathcal{F}}_{W}, \widetilde{\mu}\right)$ has measure zero:

$$
\mu_{\mathbb{W}}(U)=\mu\left(\psi_{\mathbb{W}}^{-1}(U)\right)=\mu\left(\left\{x \in \Omega: \psi_{\mathbb{W}}(x) \notin L^{1}\left(\widetilde{\Omega}, \widetilde{\mathcal{F}}_{W}, \widetilde{\mu}\right)\right\}\right)=0 .
$$

Since $\mu_{\mathbb{W}}$ has full support on $\Omega_{\mathbb{W}}$, the open set $U \cap \Omega_{\mathbb{W}} \subseteq \Omega_{\mathbb{W}}$ is empty, showing that $\Omega_{\mathbb{W}} \subseteq L^{1}\left(\widetilde{\Omega}, \widetilde{\mathcal{F}}_{W}, \widetilde{\mu}\right)$. 
Let $N=\psi_{\mathbb{W}}^{-1}(V)$, where as above $V=\widetilde{B} \backslash \Omega_{\mathbb{W}}$. We have seen that $\mu(N)=$ 0 . Furthermore, for $A \in \mathcal{G}_{\mathbb{W}}, A \subseteq \Omega_{\mathbb{W}}$ and hence $\psi_{\mathbb{W}}^{-1}(A) \subseteq \Omega \backslash N$. Finally, $\mu\left(\psi_{\mathbb{W}}^{-1}(A)\right)=\mu_{\mathbb{W}}(A)$ by the definition of $\mu_{\mathbb{W}}$. Fix some $f \in \Omega_{\mathbb{W}}$. On $N \times(\Omega \backslash N)$, change $W(x, y)$ to $f(y)$, and change it on $(\Omega \backslash N) \times N$ to make it symmetric. Finally, change it to 0 on $N \times N$, and on $N$, change $S$ to $f\left(\Omega_{\infty}\right)$. Clearly it is still the case that $W$ and $S$ are measurable and $W(x, \cdot)$ integrable for every $x$. We have changed $W$ and $S$ on a set of measure zero, so $\Omega_{\mathbb{W}}$ and $\mu_{\mathbb{W}}$ did not change, and we now have that $\psi_{\mathbb{W}}$ is an everywhere defined, measure-preserving map from $(\Omega, \mathcal{F}, \mu)$ to $\left(\Omega_{\mathbb{W}}, \mathcal{G}_{\mathbb{W}}, \mu_{\mathbb{W}}\right)$.

To complete the proof, we need to show that $\psi_{\mathbb{W}}^{-1}\left(\mathcal{G}_{\mathbb{W}}\right)=\mathcal{F}_{\mathbb{W}}$. To this end, we note that for each open $A \subseteq L^{1}\left(\widetilde{\Omega}, \widetilde{\mathcal{F}}_{W}, \widetilde{\mu}\right), \psi_{\mathbb{W}}^{-1}(A)=\psi_{\mathbb{W}}^{-1}\left(A \cap \Omega_{\mathbb{W}}\right)$, which shows that $\psi_{\mathbb{W}}^{-1}(\widetilde{\mathcal{G}})=\psi_{\mathbb{W}}^{-1}\left(\mathcal{G}_{\mathbb{W}}\right)$. This proves the last claim.

Lemma 8.13. Let $\Omega_{\mathbb{W}}$ and $\mu_{\mathbb{W}}$ be as defined above, and let $\mathcal{G}_{\mathbb{W}}$ be the Borel $\sigma$ algebra over $\Omega_{\mathbb{W}}$. If $\mathbb{W}$ is locally finite, $W(x, \cdot)$ is integrable for all $x \in \Omega$, and $\mu(\Omega \backslash \operatorname{dsupp} \mathbb{W})<\infty$, then $\left(\Omega_{\mathbb{W}}, \mathcal{G}_{\mathbb{W}}, \mu_{\mathbb{W}}\right)$ is $\sigma$-finite.

Remark 8.14. Note that the condition $\mu(\Omega \backslash$ dsupp $\mathbb{W})<\infty$ is necessary, since otherwise the point $\{(0,0)\}$ becomes an atom with infinite measure.

Proof. By Proposition 8.12, we can change $W$ on a set of measure zero such that it still satisfies the conditions of the lemma, and $\psi_{\mathbb{W}}$ becomes a measure-preserving map from $(\Omega, \mathcal{F}, \mu)$ to $\left(\Omega_{\mathbb{W}}, \mathcal{G}_{\mathbb{W}}, \mu_{\mathbb{W}}\right)$. Let $\Omega_{0} \subset L^{1}(\Omega, \mathcal{F}, \mu) \times \mathbb{R}$ be the subspace consisting of just the origin, i.e., $\Omega_{0}=\{(0,0)\}$. Then

$$
\mu_{\mathbb{W}}\left(\Omega_{0}\right)=\mu\left(\psi_{\mathbb{W}}^{-1}\left(\Omega_{0}\right)\right)=\mu\left(\left\{x \in \Omega:\left\|\psi_{\mathbb{W}}(x)\right\|_{1}=0\right\}\right)=\mu(\Omega \backslash \operatorname{dsupp} \mathbb{W})<\infty .
$$

Next define $\Omega_{n}=\left\{(f, c) \in \Omega_{\mathbb{W}}:\|f\|_{1}+c \geq 1 / n\right\}$ for $n \geq 1$. Then

$\mu_{\mathbb{W}}\left(\Omega_{n}\right)=\mu\left(\left\{x \in \Omega:\left\|\psi_{\mathbb{W}}(x)\right\|_{1}+S(x) \geq 1 / n\right\}\right)=\mu\left(\left\{x \in \Omega: D_{\mathbb{W}}(x) \geq 1 / n\right\}\right)<\infty$.

Here the last inequality follows from Proposition 2.4. Since $\Omega_{\mathbb{W}}=\Omega_{0} \cup \Omega_{1} \cup \ldots$, this proves that $\mu_{\mathbb{W}}$ is $\sigma$-finite.

Next, we would like to show that we can define a graphex $\widehat{\mathbb{W}}$ on $\Omega_{\mathbb{W}}$ such that its pullback is equal almost everywhere to $\mathbb{W}$. Using Proposition 8.12 , we can without loss of generality assume that $\psi_{\mathbb{W}}$ is a measure-preserving map from $(\Omega, \mathcal{F}, \mu)$ to $\left(\Omega_{\mathbb{W}}, \mathcal{G}_{\mathbb{W}}, \mu_{\mathbb{W}}\right)$. By Lemma 3.2 , this implies that the map $\psi_{\mathbb{W}}^{*}: L^{1}\left(\Omega_{\mathbb{W}}, \mathcal{G}_{\mathbb{W}}, \mu_{\mathbb{W}}\right) \rightarrow$ $L^{1}\left(\Omega, \mathcal{F}_{\mathbb{W}}, \mu\right)$ with $f \mapsto f^{\psi_{\mathbb{W}}}$ and $\mathcal{F}_{\mathbb{W}}=\psi_{\mathbb{W}}^{-1}\left(\mathcal{G}_{\mathbb{W}}\right)$ is an isometric isomorphism. Note that this in particular implies that $\psi_{\mathbb{W}}^{*}$ and $\left(\psi_{\mathbb{W}}^{*}\right)^{-1}$ are continuous, and hence measurable.

Now, since $\Omega_{\mathbb{W}}$ is a separable metric space, $L^{1}\left(\Omega_{\mathbb{W}}, \mathcal{G}_{\mathbb{W}}, \mu_{\mathbb{W}}\right)$ is separable, so there exists an evaluation map

$$
\Phi: L^{1}\left(\Omega_{\mathbb{W}}, \mathcal{G}_{\mathbb{W}}, \mu_{\mathbb{W}}\right) \times \Omega_{\mathbb{W}} \rightarrow \mathbb{R}
$$

such that for every $\alpha \in L^{1}\left(\Omega_{\mathbb{W}}, \mathcal{G}_{\mathbb{W}}, \mu_{\mathbb{W}}\right)$ and almost every $g \in \Omega_{\mathbb{W}}, \alpha(g)=\Phi(\alpha, g)$. Note that by definition, we also have that for every fixed $\alpha$ and almost every $y \in \Omega$, $\psi_{\mathbb{W}}^{*}(\alpha)(y)=\alpha\left(\psi_{\mathbb{W}}(y)\right)=\Phi\left(\alpha, \psi_{\mathbb{W}}(y)\right)$.

By Proposition $8.12, \Omega_{\mathbb{W}} \subseteq L^{1}\left(\widetilde{\Omega}, \widetilde{\mathcal{F}}_{\mathbb{W}}, \widetilde{\mu}\right)=L^{1}\left(\Omega, \mathcal{F}_{\mathbb{W}}, \mu\right) \times \mathbb{R}$, which means that $\left(\psi_{\mathbb{W}}^{*}\right)^{-1}\left(\left.f\right|_{\Omega}\right)$ is well defined for all $f \in \Omega_{\mathbb{W}}$. We therefore may define

$$
\widehat{W}_{0}(f, g)=\Phi\left(\left(\psi_{\mathbb{W}}^{*}\right)^{-1}\left(\left.f\right|_{\Omega}\right), g\right)
$$


and

$$
\widehat{W}(f, g)=\frac{1}{2}\left(\widehat{W}_{0}(f, g)+\widehat{W}_{0}(g, f)\right) .
$$

Since $\left(\psi_{W}^{*}\right)^{-1}$ is measurable, $\widehat{W}_{0}$ and hence $\widehat{W}$ is measurable.

Suppose $\psi_{\mathbb{W}}(x) \in \Omega_{\mathbb{W}}$. Then, noting that for all $x,\left.\psi_{\mathbb{W}}(x)\right|_{\Omega}=\psi_{W}(x)$, we have for almost all $y$,

$$
\begin{aligned}
\widehat{W}_{0}\left(\psi_{\mathbb{W}}(x), \psi_{\mathbb{W}}(y)\right) & =\Phi\left(\left(\psi_{\mathbb{W}}^{*}\right)^{-1}\left(\left.\psi_{\mathbb{W}}(x)\right|_{\Omega}\right), \psi_{\mathbb{W}}(y)\right) \\
& =\psi_{\mathbb{W}}^{*}\left(\left(\psi_{\mathbb{W}}^{*}\right)^{-1}\left(\left.\psi_{\mathbb{W}}(x)\right|_{\Omega}\right)\right)(y)=\psi_{W}(x)(y)=W(x, y),
\end{aligned}
$$

where the third and the fourth terms are only defined for almost all $y$. Thus $\widehat{W}_{0}^{\psi_{W}}$ and hence $\widehat{W}^{\psi_{W}}$ is equal to $W$ almost everywhere on $\Omega \times \Omega$. We also define $\widehat{S}(f)=f\left(\Omega_{\infty}\right)$, which gives us that for $x \in \Omega$,

$$
\widehat{S}\left(\psi_{\mathbb{W}}(x)\right)=\psi_{\mathbb{W}}(x)\left(\Omega_{\infty}\right)=S(x),
$$

implying that $S^{\psi_{\mathrm{W}}}=S$. Finally, we take $\widehat{I}=I$, giving us a graphex $\widehat{\mathbb{W}}=$ $\left(\widehat{W}, \widehat{S}, \widehat{I}, \Omega_{\mathbb{W}}\right)$ such that $\widehat{\mathbb{W}} \psi_{\mathbb{W}}=\mathbb{W}$ almost everywhere.

Note that this implies in particular that $\widehat{\mathbb{W}}$ inherits the local finiteness property from $\mathbb{W}$, so $\widehat{\mathbb{W}}$ is a bona fide graphex over the $\sigma$-finite Borel space $\left(\Omega_{W}, \mathcal{G}_{W}, \mu_{W}\right)$.

Note that the requirement that $\widehat{W}^{\psi_{\mathrm{W}}}=W$ and $\widehat{S}^{\psi_{\mathrm{W}}}=S$ almost everywhere uniquely determines $\widehat{\mathbb{W}}$ up to changes on a set of measure zero. Indeed, if $\widehat{W}^{\prime}$ is another graphon with $\widehat{W}^{\prime} \psi_{\mathrm{W}}=\widehat{W}^{\psi_{\mathrm{W}}}(\mu \times \mu)$-almost everywhere, then by the definition of pullbacks and the definition of $\mu_{\mathbb{W}}$, the equality $\widehat{W}^{\prime}=\widehat{W}$ must hold $\left(\mu_{\mathbb{W}} \times \mu_{\mathbb{W}}\right)$-almost everywhere. Similarly, if $\widehat{S}^{\prime}$ is another function with $\widehat{S}^{\prime} \psi_{\mathbb{W}}=\widehat{S}^{\psi_{W}}$ $\mu$-almost everywhere, then $\widehat{S}^{\prime}=\widehat{S} \mu_{\mathbb{W}}$-almost everywhere. Also by definition we must have $\widehat{I}^{\prime}=\widehat{I}$.

On the other hand, suppose we have two graphexes $\mathbb{W}_{1}$ and $\mathbb{W}_{2}$ on the same space $\Omega$ with $W_{1}=W_{2}$ almost everywhere, $S_{1}=S_{2}$ almost everywhere, and $I_{1}=I_{2}$. We have seen that $\Omega_{\mathbb{W}_{1}}=\Omega_{\mathbb{W}_{2}}$ and $\mu_{\mathbb{W}_{1}}=\mu_{\mathbb{W}_{2}}$. Since their pullbacks are equal almost everywhere, we must have $\widehat{W}_{1}=\widehat{W}_{2}$ almost everywhere for any choices of $\widehat{W}_{1}$ and $\widehat{W}_{2}$, and $\widehat{S}_{1}=\widehat{S}_{2}$ and $\widehat{I}_{1}=\widehat{I}_{2}$ by definition.

Finally, if the graphex $\mathbb{W}$ only has the property that $W(x, \cdot)$ is integrable for almost every $x$, we can still define $\Omega_{\mathbb{W}}$ and $\mu_{\mathbb{W}}$ in the same way, and find a $\widehat{W}$ such that the pullback is defined almost everywhere on $\Omega \times \Omega$ and equal to $W$ almost everywhere. Again, it is easy to see that we obtain the same $\Omega_{\mathbb{W}}$ and $\mu_{\mathbb{W}}$ if we first modify $W$ on a set of measure zero to make $W(x, \cdot)$ integrable for every $x$, and any choice of $\widehat{W}$ will be equal almost everywhere. Therefore, this construction gives a graphex $\widehat{\mathbb{W}}$ on $\left(\Omega_{\mathbb{W}}, \mathcal{G}_{\mathbb{W}}, \mu_{\mathbb{W}}\right)$ for any graphex $\mathbb{W}$.

Next, we show the following:

Lemma 8.15. For $i=1,2$, let $\mathbb{W}_{i}=\left(W_{i}, S_{i}, I_{i}, \boldsymbol{\Omega}_{i}\right)$ be graphexes with $\boldsymbol{\Omega}_{i}=$ $\left(\Omega_{1}, \mathcal{F}_{i}, \mu_{i}\right)$ and $\mu_{i}\left(\Omega_{i} \backslash\right.$ dsupp $\left.W_{i}\right)=0$. Suppose that there exists a measure-preserving map $\phi: \Omega_{1} \rightarrow \Omega_{2}$ such that $\mathbb{W}_{1}=\mathbb{W}_{2}^{\phi}$ almost everywhere. Extend $\phi$ to $\widetilde{\phi}: \widetilde{\Omega}_{1} \rightarrow \widetilde{\Omega}_{2}$ by $\widetilde{\phi}\left(\Omega_{1, \infty}\right)=\Omega_{2, \infty}$. Then the map $\widetilde{\phi}^{*}: L^{1}\left(\widetilde{\boldsymbol{\Omega}}_{2}\right) \rightarrow L^{1}\left(\widetilde{\boldsymbol{\Omega}}_{1}\right)$ defined by $f \mapsto f \circ \widetilde{\phi}$ restricts to a map $\Omega_{\mathbb{W}_{2}} \rightarrow \Omega_{\mathbb{W}_{1}}$, which is an isometric measure-preserving bijection between $\left(\Omega_{\mathbb{W}_{2}}, \mathcal{G}_{\mathbb{W}_{2}}, \mu_{\mathbb{W}_{2}}\right)$ and $\left(\Omega_{\mathbb{W}_{1}}, \mathcal{G}_{\mathbb{W}_{1}}, \mu_{\mathbb{W}_{1}}\right)$, and $\widehat{\mathbb{W}}_{2}=\widehat{\mathbb{W}}_{1}^{\phi^{*}}$ almost everywhere, for any choices of $\widehat{\mathbb{W}}_{1}$ and $\widehat{\mathbb{W}}_{2}$. 
Proof. By the remarks before the lemma, we may assume that $\mathbb{W}_{1}=\mathbb{W}_{2}^{\phi}$ everywhere, not just almost everywhere, and $W_{1}(x, \cdot)$ and $W_{2}\left(x^{\prime}, \cdot\right)$ are always integrable. Since $\phi$ and thus $\widetilde{\phi}$ is measure preserving, $\widetilde{\phi}^{*}$ is isometric and injective from $L^{1}\left(\widetilde{\Omega}_{2}, \widetilde{\mathcal{F}}_{2}, \widetilde{\mu}_{2}\right)$ to $L^{1}\left(\widetilde{\Omega}_{1}, \widetilde{\phi}^{-1}\left(\widetilde{\mathcal{F}}_{2}\right), \widetilde{\mu}_{2}\right)$ by Lemma 3.2 . If $x \in \Omega_{1}$, then for almost every $y \in \Omega_{1}$ (note that the first two terms below are only defined for almost every $y$ ),

$$
\left(\phi^{*} \circ \psi_{W_{2}} \circ \phi\right)(x)(y)=\left(\psi_{W_{2}} \circ \phi\right)(x)(\phi(y))=W_{2}(\phi(x), \phi(y))=W_{1}(x, y) .
$$

Therefore $\phi^{*} \circ \psi_{W_{2}} \circ \phi=\psi_{W_{1}}$ a.e. Furthermore,

$$
\left(\mathrm{id} \circ S_{2} \circ \phi\right)(x)=S_{2}(\phi(x))=S_{1}(x),
$$

which implies that $\left(\operatorname{id} \circ S_{2} \circ \phi\right)=S_{1}$. Since $\widetilde{\phi}^{*}=\phi^{*} \times$ id and $\psi_{\mathbb{W}_{i}}=\psi_{W_{i}} \times S$, this implies that $\widetilde{\phi}^{*} \circ \psi_{\mathbb{W}_{2}} \circ \phi=\psi_{\mathbb{W}_{1}}$ almost everywhere. Now let $A \subseteq L^{1}\left(\widetilde{\Omega}_{1}\right)$ be Borel measurable. Then

$$
\begin{aligned}
& \mu_{\mathbb{W}_{2}}\left(\left(\widetilde{\phi}^{*}\right)^{-1}(A)\right)=\mu_{2}\left(\psi_{\mathbb{W}_{2}}^{-1}\left(\left(\widetilde{\phi}^{*}\right)^{-1}(A)\right)\right) \\
& \quad=\mu_{1}\left(\phi^{-1}\left(\psi_{\mathbb{W}_{2}}^{-1}\left(\left(\widetilde{\phi}^{*}\right)^{-1}(A)\right)\right)\right)=\mu_{1}\left(\psi_{\mathbb{W}_{1}}^{-1}(A)\right)=\mu_{\mathbb{W}_{1}}(A) .
\end{aligned}
$$

So $\phi^{*} \times$ id: $L^{1}\left(\boldsymbol{\Omega}_{2}\right) \times \mathbb{R} \rightarrow L^{1}\left(\boldsymbol{\Omega}_{1}\right) \times \mathbb{R}$ is a measure preserving isometry. Since it is an isometry, in particular, it is continuous. Thus, $\left(\phi^{*} \times \text { id }\right)^{-1}\left(L^{1}\left(\boldsymbol{\Omega}_{1}\right) \times \mathbb{R} \backslash \Omega_{\mathbb{W}_{1}}\right)$ is an open set with measure zero, so it is disjoint from $\Omega_{\mathbb{W}_{2}}$. This implies that $\phi^{*}$ restricts to a measure-preserving injection $\Omega_{\mathbb{W}_{2}} \rightarrow \Omega_{\mathbb{W}_{1}}$. Since $\Omega_{\mathbb{W}_{2}}$ is complete, $\left(\phi^{*} \times\right.$ id $)\left(\Omega_{\mathbb{W}_{2}}\right)$ is a complete subset of $\Omega_{\mathbb{W}_{1}}$, which is itself complete. Therefore $\phi^{*}\left(\Omega_{\mathbb{W}_{2}}\right)$ is a closed subset of $\Omega_{\mathbb{W}_{1}}$. However, we also have that

$\mu_{\mathbb{W}_{1}}\left(\Omega_{\mathbb{W}_{1}} \backslash \phi^{*}\left(\Omega_{\mathbb{W}_{2}}\right)\right)=\mu_{\mathbb{W}_{2}}\left(\left(\phi^{*}\right)^{-1}\left(\Omega_{\mathbb{W}_{1}} \backslash \phi^{*}\left(\Omega_{\mathbb{W}_{2}}\right)\right)\right)=\mu_{\mathbb{W}_{2}}\left(\left(\phi^{*}\right)^{-1}\left(\Omega_{\mathbb{W}_{1}}\right) \backslash \Omega_{\mathbb{W}_{2}}\right)=0$.

But $\Omega_{\mathbb{W}_{1}} \backslash \phi^{*}\left(\Omega_{\mathbb{W}_{2}}\right)$ is an open subset of $\Omega_{\mathbb{W}_{1}}$ of measure 0 , which means it must be the empty set because $\mu_{\mathbb{W}_{1}}$ has full support in $\Omega_{\mathbb{W}_{1}}$. Therefore, $\phi^{*}: \Omega_{\mathbb{W}_{2}} \rightarrow \Omega_{\mathbb{W}_{1}}$ is a measure-preserving isometry of metric measure spaces.

Now, we want to show that $\widehat{W}_{1}^{\widetilde{\phi}^{*}}=\widehat{W}_{2}$. We have that almost everywhere on $\Omega_{1} \times \Omega_{1}$,

$$
\left(\left(\widehat{W}_{1}^{\tilde{\phi}^{*}}\right)^{\psi_{\mathbb{W}_{2}}}\right)^{\phi}=\widehat{W}_{1}^{\widetilde{\phi}^{*} \circ \psi_{\mathbb{W}_{2}} \circ \phi}=\widehat{W}_{1}^{\psi_{\mathbb{W}_{1}}}=W_{1}=W_{2}^{\phi} .
$$

Therefore $\left(\widehat{W}_{1}^{\phi^{*}}\right)^{\psi_{W_{2}}}=W_{2}$ almost everywhere, but then $\widehat{W}_{1}^{\phi^{*}}=\widehat{W}_{2}$ almost everywhere. By definition, we also have $\widehat{S}_{1}^{\phi^{*}}=\widehat{S}_{2}$, and $\widehat{I}_{1}=I_{1}=I_{2}=\widehat{I}_{2}$.

Now, suppose $\mathbb{W}_{1}$ and $\mathbb{W}_{2}$ are equivalent. Then their restrictions to their respective degree supports are also equivalent. By Corollary 8.7, there exists a chain of pullbacks that link $\mathbb{W}_{1}$ and $\mathbb{W}_{2}$. We have seen that if a graphex is a pullback of another, then the construction above yields an isomorphism between the corresponding graphexes, up to almost everywhere changes. This clearly extends to chains of pullbacks; thus, we may find an isomorphism between $\Omega_{\mathbb{W}_{1}}$ and $\Omega_{\mathbb{W}_{2}}$ so that $\widehat{\mathbb{W}}_{1}$ and $\widehat{\mathbb{W}}_{2}$ are equal almost everywhere. We can extend the map $\psi_{\mathbb{W}_{i}}: \Omega_{i} \rightarrow \Omega_{\mathbb{W}_{i}}$, which is defined almost everywhere, to be defined everywhere, by mapping the rest of the points in $\Omega_{i}$ to an arbitrary point.

\section{UNIFORM INTEGRABILITY AND UNIFORM TAIL REGULARITY}

9.1. Uniform integrability. The goal of this subsection is to prove Theorem 2.26. Before doing this we establish that several alternative definitions of uniform integrability are equivalent to Definition 2.25. 
Theorem 9.1. Given a set of integrable graphexes $\mathcal{S}$, the following are equivalent.

(1) $\mathcal{S}$ is uniformly integrable.

(2) The graphexes in $\mathcal{S}$ have uniformly bounded $\|\cdot\|_{1}$-norms, and for every $\varepsilon>0$, there exists a $D$ such that for all $\mathbb{W} \in \mathcal{S},\|\mathbb{W}\|_{1}-\|\mathbb{W} \leq D\|_{1}<\varepsilon$.

(3) For any $T>0$, the random variables $E\left(G_{T}(\mathbb{W})\right)$ with $\mathbb{W} \in \mathcal{S}$ are uniformly integrable.

(4) There exists $T>0$ such that the random variables $E\left(G_{T}(\mathbb{W})\right)$ with $\mathbb{W} \in \mathcal{S}$ are uniformly integrable.

Proof. Throughout this proof, let $\Omega_{>D}, \Omega_{\leq D}, \mathbb{W}_{>D}, \mathbb{W}_{\leq D}$, etc, be defined as before. Let us first show $(1) \Rightarrow(2)$. We have that

$$
\begin{aligned}
\left\|\mathbb{W}_{1}\right\|_{1}-\left\|\mathbb{W}_{\leq D}\right\|_{1} & =2 \int_{\Omega_{>D}} S(x) d \mu(x)+2 \int_{\Omega_{>D} \times \Omega_{\leq D}} W(x, y) d \mu(x) d \mu(y) \\
& +\int_{\Omega_{>D} \times \Omega_{>D}} W(x, y) d \mu(x) d \mu(y) \\
& =2 \int_{\Omega_{>D}} D_{\mathbb{W}}(x) d \mu(x)-\int_{\Omega_{>D} \times \Omega_{>D}} W(x, y) d \mu(x) d \mu(y) \\
& \leq 2 \int_{\Omega} D_{\mathbb{W}} 1_{D_{\mathbb{W}}>D} d \mu .
\end{aligned}
$$

Therefore, taking $D$ for $\varepsilon / 2$ in uniform integrability gives a good $D$ for $\varepsilon$ in (2).

To show that $(2) \Rightarrow(3)$, we first show that if a set of graphexes has uniformly bounded marginals, then the set of random variables is uniformly integrable. Let $E_{T}$ be the random variable for a fixed $\mathbb{W} \in \mathcal{S}$ that gives the number of edges of $G_{T}(\mathbb{W})$. Recall that by Lemma $4.3, E_{T}$ has expectation $T^{2}\|\mathbb{W}\|_{1} / 2$ and variance $T^{2}\|\mathbb{W}\|_{1} / 2+T^{3}\left\|D_{\mathbb{W}}\right\|_{2}^{2}$. Let $C$ be a bound on $\|\mathbb{W}\|_{1}$ for $\mathbb{W} \in \mathcal{S}$. We then have that for any $K>T^{2}\|\mathbb{W}\|_{1} / 2$,

$$
\mathbb{P}\left[E_{T}>K\right] \leq \frac{T^{2}\|\mathbb{W}\|_{1} / 2+T^{3}\left\|D_{\mathbb{W}}\right\|_{2}^{2}}{\left(K-T^{2}\|\mathbb{W}\|_{1} / 2\right)^{2}} \leq \frac{T^{2} C / 2+T^{3} C D}{\left(K-T^{2} C / 2\right)^{2}} .
$$

If $K \geq T^{2} C$, then this gives

$$
\mathbb{P}\left[E_{T}>K\right] \leq \frac{T^{2} C / 2+T^{3} C D}{\left(K-T^{2} C / 2\right)^{2}} \leq \frac{T^{2} C / 2+T^{3} C D}{(K / 2)^{2}}=\frac{2 T^{2} C+4 T^{3} C D}{K^{2}} .
$$

Therefore, for $K_{0} \geq T^{2} C$,

$$
\begin{aligned}
\mathbb{E}\left[E_{T} 1_{E_{T}>K_{0}}\right] & =\sum_{K=K_{0}+1}^{\infty} \mathbb{P}\left[E_{T} \geq K\right] \\
& \leq \sum_{K=K_{0}+1}^{\infty} \frac{2 T^{2} C+4 T^{3} C D}{K^{2}} \leq \frac{2 T^{2} C+4 T^{3} C D}{K_{0}}
\end{aligned}
$$

Suppose now that instead of uniformly bounded marginals, we have only (2). For $D>0$, let $E_{T, D}$ be the number of edges that either have both endpoints labeled with a vertex in $\Omega_{\leq D}$, one endpoint is labeled with a vertex in $\Omega_{\leq D}$ and the edge is generated as a star from that vertex, or the edge is a dust edge. We then have that 
for all $D>0$,

$$
\begin{aligned}
\mathbb{E}\left[E_{T} 1_{E_{T}>2 K_{0}}\right]= & \mathbb{E}\left[E_{T} 1_{E_{T}>2 K_{0}, E_{T, D}>K_{0}}\right]+\mathbb{E}\left[E_{T} 1_{E_{T}>2 K_{0}, E_{T, D} \leq K_{0}}\right] \\
\leq & \mathbb{E}\left[E_{T}-E_{T, D}\right]+\mathbb{E}\left[E_{T, D} 1_{E_{T, D}>K_{0}}\right] \\
& \quad+\mathbb{E}\left[E_{T, D} 1_{E_{T, D} \leq K_{0}, E_{T}-E_{T, D}>K_{0}}\right] \\
\leq & \mathbb{E}\left[E_{T}-E_{T, D}\right]+\frac{2 T^{2} C+4 T^{3} C D}{K_{0}}+K_{0} \mathbb{P}\left[E_{T}-E_{T, D}>K_{0}\right] \\
\leq & 2 \mathbb{E}\left[E_{T}-E_{T, D}\right]+\frac{2 T^{2} C+4 T^{3} C D}{K_{0}},
\end{aligned}
$$

provided $K_{0} \geq T^{2} C$. Condition (2) now implies that for any $\varepsilon>0$, there exists a $D$ such that $\mathbb{E}\left[E_{T, D}-E_{T}\right]<\varepsilon$. Given such a $D$, we choose $K_{0}$ in such a way that the last term in the above bound is at most $\varepsilon$, implying that for each $\varepsilon>0$ we can find a $K_{0}$ such that $\mathbb{E}\left[E_{T} 1_{E_{T}>2 K_{0}}\right] \leq 3 \varepsilon$. This proves that the set of random variables $E_{T}$ are indeed uniformly integrable.

It is clear that (3) implies (4). Suppose now that (4) holds. Since the expectation of $E_{T}$ is $T^{2}\|\mathbb{W}\|_{1} / 2,\|\mathbb{W}\|_{1}$ must be uniformly bounded for $\mathbb{W} \in \mathcal{S}$. Let $C$ be an upper bound. Suppose that (1) is false. Then there exists a fixed $\varepsilon>0$, such that for any $D$, there exists a graphex $\mathbb{W} \in \mathcal{S}$ such that

$$
\int_{\Omega_{>D}} D_{\mathbb{W}} 1_{D_{\mathbb{W}}>D} d \mu \geq \varepsilon
$$

Since $\mathbb{E}\left[D_{\mathbb{W}}\right] \leq\|\mathbb{W}\|_{1} \leq C$, we have that $\mu\left(\Omega_{>D}\right) \leq C / D$. By taking $D$ large enough, we can assume that $C / D \leq D / 2$. Let $F_{T, D}$ be the number of edges in $G_{T}(\mathbb{W})$ that have exactly one endpoint in $\Omega_{>D}$. Then

$$
\mathbb{E}\left[F_{T, D}\right] \geq \int_{\Omega_{>D}} T^{2}\left(D_{\mathbb{W}}(x)-C / D\right) d \mu(x) \geq T^{2} \int_{\Omega_{>D}}\left(D_{\mathbb{W}}(x) / 2\right) d \mu(x) \geq T^{2} \varepsilon / 2 .
$$

If there are no points sampled in $\Omega_{>D}$, then $F_{T, D}=0$. Conditioned on there being at least one point sampled in $\Omega_{>D}$, the number of neighbors of a point whose feature is $x \in \Omega_{>D}$ is a Poisson random variable with mean equal to $T D_{\mathbb{W}}(x) / 2 \geq T D / 2$. Therefore,

$$
\mathbb{E}\left[F_{T, D} \mid F_{T, D}>0\right] \geq T D / 2
$$

We also have that

$$
\mathbb{E}\left[F_{T, D} 1_{F_{T, D} \leq T D / 4} \mid F_{T, D}>0\right] \leq T D / 4
$$

Therefore,

$$
\mathbb{E}\left[F_{T, D} 1_{F_{T, D}>T D / 4} \mid F_{T, D}>0\right] \geq \frac{1}{2} \mathbb{E}\left[F_{T, D} \mid F_{T, D}>0\right] .
$$

We then have

$$
\begin{aligned}
\mathbb{E}\left[F_{T, D} 1_{F_{T, D}>T D / 4}\right] & =\mathbb{E}\left[F_{T, D} 1_{F_{T, D}>T D / 4} \mid F_{T, D}>0\right] \mathbb{P}\left[F_{T, D}>0\right] \\
& \geq \frac{1}{2} \mathbb{E}\left[F_{T, D} \mid F_{T, D}>0\right] \mathbb{P}\left[F_{T, D}>0\right]=\frac{1}{2} \mathbb{E}\left[F_{T, D}\right] \geq T^{2} \varepsilon / 4
\end{aligned}
$$

Since $D$ can be arbitrary (above some $D_{0}$ ), this contradicts Condition (4).

Theorem 2.26 is an easy corollary of Theorem 9.1. 
Proof of Theorem 2.26. By Theorem 2.18, $\mathbb{W}_{n}$ is GP-convergent to $\mathbb{W}$. Fix a subsequence $n_{i}$ such that $\liminf _{n \rightarrow \infty}\left\|\mathbb{W}_{n}\right\|_{1}=\lim _{i \rightarrow \infty}\left\|\mathbb{W}_{n_{i}}\right\|_{1}$, and fix $T>0$. Let $e_{i}$ be the number of edges in $G_{T}\left(\mathbb{W}_{n_{i}}\right)$, and let $e$ be the number of edges in $G_{T}(\mathbb{W})$. Following the proof of Corollary 3.10 in [4], for $\lambda>0$ define $f_{\lambda}: \mathbb{R}_{+} \rightarrow \mathbb{R}_{+}$by $f_{\lambda}(x)=x 1_{x \leq \lambda}$. Then $\mathbb{E}\left[f_{\lambda}\left(e_{i}\right)\right] \leq \mathbb{E}\left[e_{i}\right]=T^{2}\left\|\mathbb{W}_{n_{i}}\right\|_{1}$. Since $e_{i} \rightarrow e$ in distribution, $\mathbb{E}\left[f_{\lambda}(e)\right]=\lim _{i \rightarrow \infty} \mathbb{E}\left[f_{\lambda}\left(e_{i}\right)\right] \leq T^{2} \lim _{i \rightarrow \infty}\left\|\mathbb{W}_{i}\right\|_{1}=\liminf _{n \rightarrow \infty}\left\|\mathbb{W}_{n}\right\|_{1}$. The monotone convergence theorem then gives that $T^{2}\|\mathbb{W}\|_{1}=\mathbb{E}[e]=\lim _{\lambda \rightarrow \infty} \mathbb{E}\left[f_{\lambda}(e)\right] \leq$ $T^{2} \liminf _{n \rightarrow \infty}\left\|\mathbb{W}_{n}\right\|_{1}$, proving the first part of the theorem.

To prove the second part, assume first that $\mathbb{W}_{n}$ is uniformly integrable, and fix $\varepsilon>0$. By Theorem $9.1(2)$, for every $\varepsilon>0$, there exists a $D$ such that each $\mathbb{W}_{n}$ has

$$
\left|\left\|\mathbb{W}_{n}\right\|_{1}-\left\|\mathbb{W}_{n, \leq D}\right\|_{1}\right| \leq \varepsilon .
$$

Since $\mathbb{W}$ is integrable, after possibly increasing $D$, we can also assume that

$$
\|\mathbb{W}\|_{1}-\left\|\mathbb{W}_{\leq D}\right\|_{1} \mid \leq \varepsilon \text {. }
$$

Increasing $D$ further, we may also assume that $\mu\left(\left\{D_{\mathbb{W}}=D\right\}\right)=0$. By Proposition $4.6, \delta_{2 \rightarrow 2}\left(\mathbb{W}_{n, \leq D}, \mathbb{W}_{\leq D}\right) \rightarrow 0$, which implies in particular that $\left\|\mathbb{W}_{n, \leq D}\right\|_{1} \rightarrow$ $\left\|\mathbb{W}_{\leq D}\right\|_{1}$. Therefore, we can take $n_{0}$ so that if $n \geq n_{0}$, then

$$
\left|\left\|\mathbb{W}_{\leq D}\right\|_{1}-\left\|\mathbb{W}_{n, \leq D}\right\|_{1}\right| \leq \varepsilon .
$$

These three inequalities imply that if $n \geq n_{0}$, then

$$
\left|\left\|\mathbb{W}_{n}\right\|_{1}-\|\mathbb{W}\|_{1}\right| \leq 3 \varepsilon .
$$

Since $\varepsilon$ was arbitrary, this completes the proof of the first direction.

For the other direction, fix $\varepsilon$. Since $\mathbb{W}$ is integrable, there exists $D>0$ such that

$$
\left\|\mathbb{W}_{\leq D}\right\|_{1} \geq\|\mathbb{W}\|_{1}-\varepsilon / 2
$$

By increasing $D$, we can assume that $\mu\left(\left\{D_{\mathbb{W}}=D\right\}\right)=0$. By Proposition 4.6,

$$
\left\|\mathbb{W}_{n, \leq D}\right\|_{1} \rightarrow\left\|\mathbb{W}_{\leq D}\right\|_{1} .
$$

We then have that

$$
\begin{array}{r}
\limsup _{n \rightarrow \infty}\left(\left\|\mathbb{W}_{n}\right\|_{1}-\left\|\mathbb{W}_{n, \leq D}\right\|_{1}\right)=\limsup _{n \rightarrow \infty}\left(\left\|\mathbb{W}_{n}\right\|_{1}-\|\mathbb{W}\|_{1}\right)+\left(\|\mathbb{W}\|_{1}-\left\|\mathbb{W}_{\leq D}\right\|_{1}\right) \\
\left.+\left(\left\|\mathbb{W}_{\leq D}\right\|_{1}-\left\|\mathbb{W}_{n, \leq D}\right\|_{1}\right)\right) \leq \varepsilon / 2
\end{array}
$$

Therefore, there exists an $n_{0}$ such that if $n>n_{0}$, then

$$
\left\|\mathbb{W}_{n}\right\|_{1}-\left\|\mathbb{W}_{n, \leq D}\right\|_{1}<\varepsilon
$$

Since $\mathbb{W}_{1}, \mathbb{W}_{2}, \ldots, \mathbb{W}_{n_{0}}$ is a finite set of graphexes, each of which is integrable, we can increase $D$ so that the above inequality holds for each $n$, which by Theorem 9.1 means that they are uniformly integrable.

9.2. Uniform tail regularity. The goal of this subsection is to prove Theorem 2.28. Before doing this, we show that uniform tail regularity implies uniform integrability.

Lemma 9.2. Suppose that a set of graphexes consisting only of graphons is uniformly tail regular. Then the set is uniformly integrable. 
Proof. Fix $\varepsilon>0$. By the definition of tail regularity, we can find an $M<\infty$ such that for each graphon $W$ in the set there exists a subset $\Omega_{0}$ of measure at most $M$ such that $\|W\|_{1}-\left\|\left.W\right|_{\Omega_{0}}\right\|_{1} \leq \varepsilon / 3$. Note that clearly $\|W\|_{1} \leq M^{2}+\varepsilon / 3$, so in particular the set of graphons has uniformly bounded $L^{1}$ norm. Let

$$
A=\left\{x \in \Omega_{0}, D_{W}(x)>2 M\right\} .
$$

Note that for any $x \in A$,

$$
\int_{\Omega \backslash \Omega_{0}} W(x, y) d \mu(y) \geq D_{W}(x)-\int_{\Omega_{0}} W(x, y) d \mu(y) \geq D_{W}(x)-M \geq M,
$$

which implies that

$$
\mu(A) \leq \frac{1}{M} \int_{A \times \Omega \backslash \Omega_{0}} W d \mu^{2} \leq \frac{1}{M} \int_{\Omega \backslash \Omega_{0}} D_{W} d \mu \leq \frac{\varepsilon}{3 M} .
$$

We then have

$$
\begin{aligned}
\int_{\Omega} D_{W} 1_{D_{W}>2 M} d \mu & \leq \int_{\Omega \backslash \Omega_{0}} D_{W} d \mu+\int_{A} D_{W} d \mu \\
& =\int_{\Omega \backslash \Omega_{0}} D_{W} d \mu+\int_{A \times\left(\Omega \backslash \Omega_{0}\right)} W(x, y) d \mu(x) d \mu(y) \\
& \quad+\int_{A \times \Omega_{0}} W(x, y) d \mu(x) d \mu(y) \\
& \leq 2 \int_{\Omega \backslash \Omega_{0}} D_{W} d \mu+\int_{A \times \Omega_{0}} W(x, y) d \mu(x) d \mu(y) \\
& \leq 2 \varepsilon / 3+\mu(A) \mu\left(\Omega_{0}\right) \leq \varepsilon .
\end{aligned}
$$

Next, we show the following lemma. As before, $\Omega_{>\delta}$ is the set $\left\{x \in \Omega: D_{\mathbb{W}}(x)>\right.$ $\delta\}$.

Lemma 9.3. Given a set of graphons $\mathcal{S}$, the following are equivalent:

(1) The set of graphons is uniformly tail regular.

(2) The set of graphons has a uniform bound on their $\|\cdot\|_{1}$-norm, and for every $\varepsilon$, there exists a $\delta$ such that $\|W\|_{1}-\left\|\left.W\right|_{\Omega_{>\delta}}\right\|_{1} \leq \varepsilon$.

(3) The set of graphons has a uniform bound on their $\|\cdot\|_{1}$-norm, and for every $\varepsilon$, there exists a $\delta$ such that $\left\|D_{W} 1_{D_{W} \leq \delta}\right\|_{1} \leq \varepsilon$.

Corollary 9.4. Given a set of graphons $\mathcal{S}$, suppose that we replace each graphon with a pullback. Let $\mathcal{S}^{\prime}$ be the new set. Then $\mathcal{S}^{\prime}$ is uniformly tail regular if and only if $\mathcal{S}$ is.

Proof. Property (2) in Lemma 9.3 is unaffected by taking pullbacks.

Proof of Lemma 9.3. We first show that (1) implies (2). Fix $\varepsilon>0$. Take $M$ for $\varepsilon / 2$ as in the definition of uniform tail regularity, let $\delta=\varepsilon / 4 M$. Fix an arbitrary graphon $W \in \mathcal{S}$, and let $\Omega_{0}$ be a set of measure $M$ such that

$$
\|W\|_{1}-\left\|\left.W\right|_{\Omega_{0}}\right\|_{1} \leq \varepsilon / 2
$$

Note that $W$ has $L^{1}$ norm at most $M^{2}+\varepsilon / 2$, which proves that the graphs have a uniform bound on their $\|\cdot\|_{1}$-norm. Now, we have

$\|W\|_{1}-\left\|\left.W\right|_{\Omega_{>\delta}}\right\|_{1} \leq\|W\|_{1}-\left\|\left.W\right|_{\Omega_{0}}\right\|_{1}+\left\|\left.W\right|_{\Omega_{0}}\right\|_{1}-\left\|\left.W\right|_{\Omega_{0} \cap \Omega_{>\delta}}\right\|_{1} \leq \varepsilon / 2+2 \delta M \leq \varepsilon$.

This shows that (1) implies (2). 
The fact that (2) implies (1) follows from the observation that

$$
\mu\left(\Omega_{>\delta}\right)=\int d \mu(x) 1_{D_{W}(x)>\delta} \leq \int d \mu(x) \frac{D_{W}(x)}{\delta}=\frac{1}{\delta}\|W\|_{1} .
$$

Finally, (2) and (3) are equivalent by the fact that

$$
\int_{\Omega \times \Omega \backslash \Omega_{>\delta}} W \leq \int_{\Omega \times \Omega} W-\int_{\Omega_{>\delta} \times \Omega_{>\delta}} W \leq 2 \int_{\Omega \times \Omega \backslash \Omega_{>\delta}} W .
$$

To prove Theorem 2.28 we establish three more lemmas.

Lemma 9.5. Suppose that a sequence of integrable graphons $W_{n}$ converge to a graphon $W$ in the cut metric. Then for any $D>0$ such that $\mu\left(D_{W}=D\right)=0$, the graphons $W_{n, \leq D}$ converge to $W_{\leq D}$ in the cut metric.

Proof. Let $\widetilde{\mu}_{n}$ be a coupling of trivial extensions of $W_{n}$ and $W$, and let $\widetilde{\Omega}_{n}$ be the product space on which the coupling is defined. Let $\widetilde{W}_{n}$ and $\widetilde{W}$ be the pullbacks of the trivial extensions to $\widetilde{\Omega}_{n}$, and suppose that

$$
\left\|\widetilde{W}_{n}-\widetilde{W}\right\|_{\square}<\varepsilon .
$$

Defining $A=\left\{D_{\widetilde{W}_{n}}-D_{\widetilde{W}}>0\right\}$ and $B=\left\{D_{\widetilde{W}}-D_{\widetilde{W}_{n}}>0\right\}$, we then have that

$$
\begin{aligned}
\widetilde{\mu}_{n}\left(\left\{\left|D_{\widetilde{W}_{n}}-D_{\widetilde{W}}\right|>\sqrt{\varepsilon}\right\}\right) & \leq \frac{1}{\sqrt{\varepsilon}}\left\|D_{\widetilde{W}_{n}}-D_{\widetilde{W}}\right\|_{1} \\
& =\frac{1}{\sqrt{\varepsilon}}\left(\int_{A \times \widetilde{\Omega}_{n}}\left(\widetilde{W}_{n}-\widetilde{W}\right)+\int_{B \times \widetilde{\Omega}_{n}}\left(\widetilde{W}-\widetilde{W}_{n}\right)\right)<2 \sqrt{\varepsilon} .
\end{aligned}
$$

As a consequence,

$$
\begin{aligned}
\widetilde{\mu}_{n}\left(\left\{D_{\widetilde{W}_{n}}>D,\right.\right. & \left.\left.D_{\widetilde{W}} \leq D\right\}\right) \\
& \leq \widetilde{\mu}_{n}\left(\left\{\left|D_{\widetilde{W}_{n}}-D_{\widetilde{W}}\right|>\sqrt{\varepsilon}\right\}\right)+\widetilde{\mu}_{n}\left(\left\{D-\sqrt{\varepsilon}<D_{\widetilde{W}} \leq D\right\}\right) \\
& <2 \sqrt{\varepsilon}+\mu\left(\left\{D-\sqrt{\varepsilon}<D_{W} \leq D\right\}\right) .
\end{aligned}
$$

For any $\delta>0$, we can take $\varepsilon$ small enough so that this is at most $\delta$. Similarly, we have

$$
\begin{aligned}
\widetilde{\mu}_{n}\left(\left\{D_{\widetilde{W}_{n}} \leq D,\right.\right. & \left.\left.D_{\widetilde{W}}>D\right\}\right) \\
& \leq \widetilde{\mu}_{n}\left(\left\{\left|D_{\widetilde{W}_{n}}-D_{\widetilde{W}}\right|>\sqrt{\varepsilon}\right\}\right)+\widetilde{\mu}_{n}\left(\left\{D<D_{\widetilde{W}} \leq D+\sqrt{\varepsilon}\right\}\right) \\
& <2 \sqrt{\varepsilon}+\mu\left(\left\{D<D_{W} \leq D+\sqrt{\varepsilon}\right\}\right)
\end{aligned}
$$

which is also at most $\delta$ if $\varepsilon$ is small enough.

Next we trivially extend $W_{n, \leq D}$ and $W_{\leq D}$ first to the spaces $W_{n}$ and $W$ are defined on, and then to the spaces used in the coupling $\widetilde{\mu}_{n}$. Let $\widetilde{W}_{n, \leq D}$ and $\widetilde{W}_{\leq D}$ be the pullbacks, let $\widetilde{W}_{n, \leq D}^{\prime}$ be equal to $\widetilde{W}_{n, \leq D}$ on $\left\{D_{\widetilde{W}} \leq D\right\}^{2}$ and 0 otherwise, and let $\widetilde{W}_{\leq D}^{\prime}$ be equal to $\widetilde{W}_{\leq D}$ on $\left\{D_{\widetilde{W}_{n}} \leq D\right\}^{2}$ and 0 otherwise. Then $\widetilde{W}_{n, \leq D}$ and $\widetilde{W}_{n, \leq D}^{\prime}$ differ only on $\left\{D_{\widetilde{W}_{n}} \leq D, D_{\widetilde{W}}>D\right\} \times\left\{D_{\widetilde{W}_{n}} \leq D\right\}$ and its transpose. Indeed, if $D_{\widetilde{W}_{n}}>D$ in either coordinate, then both graphons are zero, and if $D_{\widetilde{W}} \leq D$ in both coordinates, then by the definition they are the same. Since $\widetilde{W}_{n, \leq D}$ has maximum degree $D$, this implies that

$$
\left\|\widetilde{W}_{n, \leq D}-\widetilde{W}_{n, \leq D}^{\prime}\right\|_{\square} \leq\left\|\widetilde{W}_{n, \leq D}-\widetilde{W}_{n, \leq D}^{\prime}\right\|_{1} \leq 2 \widetilde{\mu}_{n}\left(\left\{D_{\widetilde{W}_{n}} \leq D, D_{\widetilde{W}}>D\right\}\right) D \leq 2 \delta D .
$$


Analogously,

$$
\left\|\widetilde{W}_{\leq D}-\widetilde{W}_{\leq D}^{\prime}\right\|_{\square} \leq 2 \delta D .
$$

Note that $\widetilde{W}_{n, \leq D}^{\prime}$ and $\widetilde{W}_{\leq D}^{\prime}$ are equal to $\widetilde{W}_{n}$ and $\widetilde{W}$, respectively, on $\left\{D_{\widetilde{W}_{n}} \leq\right.$ $\left.D, D_{\widetilde{W}} \leq D\right\}^{2}$, and zero everywhere else, which implies that $\widetilde{W}_{n, \leq D}^{\prime}-\widetilde{W}_{\leq D}^{\prime}$ is the restriction of $\widetilde{W}_{n}-\widetilde{W}$ to $\left\{D_{\mathbb{W}_{n}} \leq D, D_{\mathbb{W}} \leq D\right\}^{2}$. This implies that

$$
\left\|\widetilde{W}_{n, \leq D}^{\prime}-\widetilde{W}_{\leq D}^{\prime}\right\|_{\square} \leq\left\|\widetilde{W}_{n}-\widetilde{W}\right\|_{\square}<\varepsilon
$$

which in turn implies that

$$
\begin{aligned}
\left\|\widetilde{W}_{n, \leq D}-\widetilde{W}_{\leq D}\right\|_{\square} \leq & \left\|\widetilde{W}_{n, \leq D}-\widetilde{W}_{n, \leq D}^{\prime}\right\|_{\square}+\left\|\widetilde{W}_{n, \leq D}^{\prime}-\widetilde{W}_{\leq D}^{\prime}\right\|_{\square} \\
& +\left\|\widetilde{W}_{\leq D}^{\prime}-\widetilde{W}_{\leq D}\right\|_{\square} \\
\leq & 4 \delta D+\varepsilon .
\end{aligned}
$$

Taking $\varepsilon$ small enough, this can be made arbitrarily small, which completes the proof.

We also have the following:

Lemma 9.6. Suppose that a sequence of integrable graphons $W_{n}$ have uniformly bounded marginals, and converge to a (necessarily integrable) graphon $W$ in the cut metric. Then $W$ has the same bound on its marginals, and $\delta_{2 \rightarrow 2}\left(\mathbb{W}_{n}, \mathbb{W}\right) \rightarrow 0$.

Proof. Suppose that for each $n, D_{W_{n}} \leq D$ almost everywhere, but $D_{W}>D$ on a set of positive measure. Then there exists $D^{\prime} \geq D$ such that $\mu\left(\left\{D_{W}>D^{\prime}\right\}\right)>0$ and $\mu\left(\left\{D_{W}=D^{\prime}\right\}\right)=0$. By Lemma $9.5, W_{n, \leq D^{\prime}}$ converges to $W_{\leq D^{\prime}}$ in the cut metric, but the cut distance of $W_{n, \leq D^{\prime}}$ from $W_{n}$ is 0 , since $D_{W_{n}} \leq D$ almost everywhere. Therefore, the cut distance of $W_{\leq D^{\prime}}$ and $W$ is 0 , which is a contradiction.

Now, we have the following. Since $\left\|W_{n}\right\|_{1} \rightarrow\|W\|_{1}$, there exists a uniform bound $C$ on $\left\|W_{n}\right\|_{1}$ and $\|W\|_{1}$, which implies that $\left\|W_{n}-W\right\|_{1} \leq 2 C$. Furthermore, for any $x,\left|D_{\left|W_{n}-W\right|}(x)\right| \leq D_{W_{n}}(x)+D_{W}(x) \leq 2 D$. By Lemma 3.22 , and recalling that $\|U\|_{\otimes} \leq \sqrt{\|U\|_{\square}\|U\|_{\infty}}$, we have

$$
\begin{aligned}
\left\|W_{n}-W\right\|_{2 \rightarrow 2} & \leq\left(8\left\|W_{n}-W\right\|_{\otimes}\left\|W_{n}-W\right\|_{\infty}^{3 / 4}\left\|D_{\left|W_{n}-W\right|}\right\|_{\infty}^{3 / 2}\left\|W_{n}-W\right\|_{1}^{3 / 4}\right)^{1 / 4} \\
& \leq\left(8\left\|W_{n}-W\right\|_{\square}^{1 / 2}\left\|W_{n}-W\right\|_{\infty}^{5 / 4}\left\|D_{\left|W_{n}-W\right|}\right\|_{\infty}^{3 / 2}\left\|W_{n}-W\right\|_{1}^{3 / 4}\right)^{1 / 4} \\
& \leq\left(100 D^{3 / 2} C^{3 / 4}\left\|W_{n}-W\right\|_{\square}^{1 / 2}\right)^{1 / 4} \rightarrow 0 .
\end{aligned}
$$

Furthermore, note that

$$
\left\|D_{W_{n}}-D_{W}\right\|_{1} \leq 2 \sup _{S}\left\{\left|\int_{S} D_{W_{n}}-D_{W}\right|\right\} \leq 2\left\|W_{n}-W\right\|_{\square} .
$$

Therefore,

$$
\begin{aligned}
\left\|D_{\mathbb{W}_{n}}-D_{\mathbb{W}}\right\|_{2} & =\left\|D_{W_{n}}-D_{W}\right\|_{2} \\
& \leq \sqrt{\left\|D_{W_{n}}-D_{W}\right\|_{1}\left\|D_{W_{n}}-D_{W}\right\|_{\infty}} \leq \sqrt{4 D\left\|W_{n}-W\right\|_{\square}} \rightarrow 0 .
\end{aligned}
$$

Finally,

$$
\left\|\mathbb{W}_{n}\right\|_{1}=\left\|W_{n}\right\|_{1} \rightarrow\|W\|_{1}=\|\mathbb{W}\|_{1} .
$$

The last lemma we need to prove Theorem 2.28 is the following. 
Lemma 9.7. Suppose that $\delta_{\diamond}\left(\mathbb{W}, \mathbb{W}^{\prime}\right)=0$ for two integrable graphexes, and suppose that $\mathbb{W}=(W, 0,0, \boldsymbol{\Omega})$. Then $\mathbb{W}^{\prime}=\left(W^{\prime}, 0,0, \boldsymbol{\Omega}^{\prime}\right)$ and $\delta_{\square}\left(W, W^{\prime}\right)=0$.

Proof. Since $\delta_{\diamond}\left(\mathbb{W}, \mathbb{W}^{\prime}\right)=0, \xi(G(\mathbb{W}))$ and $\xi\left(G\left(\mathbb{W}^{\prime}\right)\right)$ have the same distribution. But, as already observed in Remark 5.4 in [17], almost surely, the dust part of $\mathbb{W}^{\prime}$ generates edges which are isolated, the star part generates edges with one vertex of degree one and a second vertex of infinite degree, and the graphon part generates edges with two endpoints of infinite degree. Since $\xi(G(\mathbb{W}))$ has no star or dust edges, $\xi\left(G\left(\mathbb{W}^{\prime}\right)\right)$ doesn't have these either, showing that $\mathbb{W}^{\prime}=\left(W^{\prime}, 0,0, \boldsymbol{\Omega}^{\prime}\right)$. Finally, since the graphon process generated by $W$ and $W^{\prime}$ have the same distribution, $\delta_{\square}\left(W, W^{\prime}\right)=0$ by Theorem 27 in [3].

We are now ready to prove Theorem 2.28.

Proof of Theorem 2.28. First, we show that if a sequence converges in the cut metric, then it converges in $\delta_{\diamond}$. We show property (2) from Proposition 4.6. Since the graphons converge in cut metric, we must have in particular that $\left\|\mathbb{W}_{n}\right\|_{1}=$ $\left\|W_{n}\right\|_{1} \rightarrow\|W\|_{1}=\|\mathbb{W}\|_{1}$, which implies that the set $\left\{\left\|\mathbb{W}_{n}\right\|\right\}_{n}$ is uniformly bounded; therefore, the sequence is tight by Corollary 4.2 (1). By Lemma 9.5, for any $D>0$ with $\mu\left(\left\{D_{W}=D\right\}\right)=0, W_{n, \leq D}$ converges to $W_{\leq D}$ in cut metric, and by Lemma 9.6, they must also converge in $\delta_{2 \rightarrow 2}$, which completes the proof that cut metric convergence implies weak kernel convergence. Since we know that any cut metric convergent sequence is uniformly tail regular, this completes that proof that (1) implies (2). It is clear that (2) is stronger than (3), so it remains to show that (3) implies (1).

To this end, we first note that uniform tail regularity implies uniformly bounded $L^{1}$ norms, which by Theorem 2.26 implies that $\mathbb{W}$ is integrable. Suppose that $\mathbb{W}$ does not consist of only a graphon part, or that $W_{n}$ does not converge to it in the cut metric. Since the sequence $W_{n}$ is uniformly tail regular, we may choose a subsequence that converges to an integrable graphon $W^{\prime}$, such that either $\delta_{\square}\left(W^{\prime}, W\right) \neq 0$, or $\mathbb{W}$ is not just a graphon. In either case, letting $\mathbb{W}^{\prime}=\left(W^{\prime}, 0,0, \boldsymbol{\Omega}^{\prime}\right)$, we have by Lemma 9.7 that $\delta_{\diamond}\left(\mathbb{W}, \mathbb{W}^{\prime}\right) \neq 0$. However, since $\delta_{\square}\left(W_{n}, W^{\prime}\right) \rightarrow 0$, we must have that $\delta_{\diamond}\left(\mathbb{W}_{n}, \mathbb{W}^{\prime}\right) \rightarrow 0$, which implies that $\delta_{\diamond}\left(\mathbb{W}^{\prime}, \mathbb{W}\right)=0$, which is a contradiction. This completes the proof that (3) implies (1), and thus we have proven the theorem.

Proof of Theorem 2.29. First, assume that $\mathbb{W}=(W, 0,0, \boldsymbol{\Omega})$, and let us prove (2). Assume first that the sequence has uniformly bounded marginals. In this case, $\delta_{2 \rightarrow 2}\left(\mathbb{W}_{n}, \mathbb{W}\right) \rightarrow 0$. Take a sequence of couplings of trivial extensions of $\mathbb{W}_{n}$ and $\mathbb{W}$ which show that their kernel distance goes to zero, let $\boldsymbol{\Omega}_{n}^{\prime}$ be the space for each $n$, and $\mathbb{W}_{n}^{\prime}$ and $\mathbb{W}^{n}$ the pulled back graphexes, and let $W_{n}^{\prime}$ and $W^{n}$ be their graphon parts. By Corollary 9.4, it is enough to prove uniform tail regularity for $W_{n}^{\prime}$. Given $\varepsilon>0$, let $\delta>0$ be such that

$$
\|W\|_{1}-\left\|\left.W\right|_{M_{\delta}}\right\|_{1} \leq \varepsilon
$$

where $M_{\delta}$ is the set

$$
M_{\delta}=\left\{x \in \Omega: D_{W}(x) \geq \delta\right\} .
$$

Let $M_{\delta}^{n}$ be the pullback to $\Omega_{n}^{\prime}$. We then have that

$$
\left\|W_{n}^{\prime}-W^{n}\right\|_{2 \rightarrow 2} \rightarrow 0 \text {. }
$$


Since $M_{\delta}^{n}$ has finite measure, this implies that

$$
\int_{M_{\delta}^{n} \times M_{\delta}^{n}} W_{n}^{\prime} \rightarrow \int_{M_{\delta} \times M_{\delta}} W
$$

Since $\left\|\mathbb{W}_{n}^{\prime}\right\|_{1}=\left\|\mathbb{W}_{n}\right\|_{1} \rightarrow\|\mathbb{W}\|_{1}=\|W\|_{1}$, this implies that

$$
\limsup _{n \rightarrow \infty}\left(\left\|W_{n}^{\prime}\right\|_{1}-\int_{M_{\delta}^{n} \times M_{\delta}^{n}} W_{n}^{\prime}\right) \leq \limsup _{n \rightarrow \infty}\left(\left\|\mathbb{W}_{n}^{\prime}\right\|_{1}-\int_{M_{\delta}^{n} \times M_{\delta}^{n}} W_{n}^{\prime}\right) \leq \varepsilon .
$$

This can be made arbitrarily small by taking $\delta$ small enough, which proves uniform tail regularity under the assumption of uniformly bounded marginals. On the other hand,

$$
\begin{aligned}
2 \limsup _{n \rightarrow \infty}\left(\left\|S_{n}\right\|_{1}+I_{n}\right) & =\limsup _{n \rightarrow \infty}\left(\left\|\mathbb{W}_{n}\right\|_{1}-\left\|W_{n}^{\prime}\right\|_{1}\right) \\
& \leq\|W\|_{1}-\lim _{n \rightarrow \infty} \int_{M_{\delta}^{n} \times M_{\delta}^{n}} W_{n}^{\prime} \leq \varepsilon,
\end{aligned}
$$

which implies that $\left\|S_{n}\right\|_{1} \rightarrow 0$ and $I_{n} \rightarrow 0$, completing the proof of (2) under the assumption of uniformly bounded marginals.

If instead of uniformly bounded marginals, we have uniform integrability, then the claims follow from the fact that for each $D>0, \delta_{2 \rightarrow 2}\left(\mathbb{W}_{n, \leq D}, \mathbb{W}_{\leq D}\right) \rightarrow 0$, and we can take $D$ large enough so that each $\int_{\Omega_{n,>D}} S_{n}$ is less than $\varepsilon$ and

$$
\int_{\Omega \times \Omega>D} W_{n}<\varepsilon
$$

and $I_{n}$ is unaffected by the restriction.

Conversely, assume (2). Let, for each $n, \mathbb{W}_{n}^{\prime}=\left(W_{n}, 0,0, \boldsymbol{\Omega}\right)$ (so we are replacing $S_{n}$ and $I_{n}$ with 0$)$. Since $\left\|S_{n}\right\|_{1} \rightarrow 0$ and $I_{n} \rightarrow 0$,

$$
\delta_{\diamond}\left(\mathbb{W}_{n}, \mathbb{W}_{n}^{\prime}\right) \rightarrow 0
$$

Indeed, clearly $\left|\left\|\mathbb{W}_{n}\right\|_{1}-\left\|\mathbb{W}_{n}^{\prime}\right\|_{1}\right| \rightarrow 0$, and for any $D>0$, we have that $\int_{\Omega_{<D}} S_{n}^{2} \leq$ $D\left\|S_{n}\right\|_{1} \rightarrow 0$. Taking $D$ large enough that $\Omega_{>D}$ has small measure, we can show that $\delta_{\diamond}\left(\mathbb{W}_{n}, \mathbb{W}_{n}^{\prime}\right)$ is arbitrarily small for large enough $n$. Now, the statement follows from Theorem 2.28; specifically, we have shown that (3) holds for the sequence $\mathbb{W}_{n}^{\prime}$, which by (2) implies that the limit is a pure graphon.

The equivalence of (2) and (3) follows from Theorem 2.28 applied to the sequence $\mathbb{W}_{n}^{\prime}$.

\section{ACKNOWLEDGEMENTS}

László Miklós Lovász thanks Microsoft Research New England for an internship in the summer of 2016, when most of the research part of this work was done. László Miklós Lovász was also supported by NSF Postdoctoral Fellowship Award DMS 1705204 for part of this work. All of us thank Svante Janson and Nina Holden for various discussions about the work presented here. 


\section{ApPENDix A. LOCAL FINITENESS}

In this appendix, we prove Proposition 2.4.

Throughout this appendix, $\boldsymbol{\Omega}=(\Omega, \mathcal{F}, \mu)$ will be a $\sigma$-finite measure space, $S: \Omega \rightarrow \mathbb{R}_{+}$will be measurable, $W: \Omega \times \Omega \rightarrow[0,1]$ will be a symmetric, measurable function, $\eta=\sum_{i} \delta_{x_{i}}$ will be a Poisson point process on $\Omega$ with intensity $\mu$, and

$$
\eta(S)=\sum_{i} S\left(x_{i}\right) \quad \text { and } \quad \eta^{2}(W)=\sum_{i \neq j} W\left(x_{i}, x_{j}\right) .
$$

We start with the following lemma, which is the analogue of Lemma A.3.6 from [19] for general measure spaces. We use $\mathbb{E}$ to denote expectations with respect to the Poisson point process and $W \circ W$ to denote the function $(x, y) \mapsto \int W(x, z) W(z, y) d \mu(z)$.

Lemma A.1. Let $\psi(x)=1-e^{-x}$. Then the following hold, with both side of the various identities being possibly infinite:

(1) $\mathbb{E}[\eta(S)]=\|S\|_{1}$ and $\mathbb{E}\left[\eta^{2}(W)\right]=\|W\|_{1}$,

(2) $\mathbb{E}[\psi(\eta(S))]=\psi\left(\|\psi(S)\|_{1}\right)$, and

(3) $\mathbb{E}\left[\left(\eta^{2}(W)\right)^{2}\right]=\|W\|_{1}^{2}+4\|W \circ W\|_{2}+2\left\|W^{2}\right\|_{1}$.

Proof. We first assume that $m=\mu(\Omega)$ is finite and $S$ is bounded. Then $\eta$ can be generated by first choosing $N$ as a Poisson random variable with rate $m$ and then choosing $x_{1}, \ldots, x_{N}$ i.i.d. according to the distribution $\frac{1}{m} \mu$. Conditioned on $N$, the expectations of $\eta(S)$ and $\eta^{2}(W)$ are $\frac{N}{m}\|S\|_{1}$ and $\frac{N(N-1)}{m^{2}}\|W\|_{1}$, respectively, and the expectation of $\psi(\eta(S))$ is

$$
\begin{aligned}
\mathbb{E}[\psi(\eta(S)) \mid N] & =1-\mathbb{E}\left[e^{-\sum_{i=1}^{N} S\left(x_{i}\right)}\right] \\
& =1-\prod_{i=1}^{N} \frac{1}{m} \int_{\Omega} d \mu\left(x_{i}\right) e^{-S\left(x_{i}\right)}=1-\left(\frac{1}{m} \int_{\Omega} d \mu(x) e^{-S(x)}\right)^{N} .
\end{aligned}
$$

Therefore,

$$
\mathbb{E}[\eta(S)]=\sum_{N=0}^{\infty} e^{-m} \frac{m^{N}}{N !} \frac{N}{m}\|S\|_{1}=\sum_{N=1}^{\infty} e^{-m} \frac{m^{N-1}}{(N-1) !}\|S\|_{1}=\|S\|_{1} .
$$

Also,

$$
\mathbb{E}\left[\eta^{2}(W)\right]=\sum_{N=0}^{\infty} e^{-m} \frac{m^{N}}{N !} \frac{N(N-1)}{m^{2}}\|W\|_{1}=\sum_{N=,}^{\infty} e^{-m} \frac{m^{N-2}}{(N-2) !}\|W\|_{1}=\|W\|_{1} .
$$

Finally,

$$
\begin{aligned}
\mathbb{E}[\psi(\eta(S))] & =\sum_{N=0}^{\infty} e^{-m} \frac{m^{N}}{N !}\left(1-\left(\frac{1}{m} \int_{\Omega} d \mu(x) e^{-S(x)}\right)^{N}\right) \\
& =1-\sum_{N=0}^{\infty} e^{-m} \frac{m^{N}}{N !}\left(\frac{1}{m} \int_{\Omega} d \mu(x) e^{-S(x)}\right)^{N} \\
& =1-\exp \left(\int_{\Omega} e^{-S(x)} d \mu(x)-m\right)=1-\exp \left(\int_{\Omega}-\psi(S(x)) d \mu(x)\right) \\
& =1-e^{\|\psi(S)\|_{1}}=\psi\left(\|\psi(S)\|_{1}\right) .
\end{aligned}
$$


To calculate the expectation of

$$
\left(\eta^{2}(W)\right)^{2}=\sum_{i \neq j} \sum_{k \neq \ell} \mathbb{E}\left[W\left(x_{i}, x_{j}\right) W\left(x_{k}, x_{\ell}\right)\right]
$$

we distinguish whether $\{i, j\}$ and $\{k, \ell\}$ intersect in 0,1 , or 2 elements, leading to the expression

$$
\begin{aligned}
\mathbb{E}\left[\left(\eta^{2}(W)\right)^{2} \mid N\right]= & \frac{N(N-1)(N-2)(N-3)}{m^{4}}\|W\|_{1}^{2} \\
& +\frac{4 N(N-1)(N-2)}{m^{3}}\|W \circ W\|_{1}+\frac{2 N(N-1)}{m^{2}}\left\|W^{2}\right\|_{1} .
\end{aligned}
$$

Taking the expectation over $N$ gives the expression in the lemma similarly. This completes the proof for spaces of finite measure and bounded functions $S$. The general case follows by the monotone convergence theorem.

Using Lemma A.1, we now prove the following proposition, which is the analogue of the relevant parts for us of Theorem A3.5 from [19] for general $\sigma$-finite measure spaces.

Proposition A.2. Let $S: \Omega \rightarrow \mathbb{R}_{+}$be measurable, and let $W: \Omega \times \Omega \rightarrow[0,1]$ be symmetric and measurable. Then the following hold:

(1) $\eta(S)<\infty$ a.s. if and only if $\|\min \{S, 1\}\|_{1}<\infty$, and

(2) $\eta^{2}(W)<\infty$ a.s. if and only if there exists a finite $D>0$ such that the following three conditions hold:

(a) $D_{W}<\infty$ almost surely,

(b) $\mu\left(\left\{x \in \Omega: D_{W}(x)>D\right\}\right)<\infty$, and

(c) $\left\|\left.W\right|_{\left\{x \in \Omega: D_{W}(x) \leq D\right\}}\right\|_{1}<\infty$.

Proof. Since $\frac{1}{2} \min \{1, x\} \leq \psi(x) \leq \min \{1, x\}$, the condition $\|\min \{S, 1\}\|_{1}<\infty$ in (1) is equivalent to the statement that $\|\psi(S)\|_{1}<\infty$, which is equivalent to the statement that $\psi\left(\|\psi(S)\|_{1}\right)<1$. By Lemma A.1 (2), this is equivalent to saying that $\mathbb{E}[\psi(\eta(S))]<1$, which holds if and only if $\eta(S)<\infty$ with positive probability. By Kolmogorov's zero-one law, we either have $\eta(S)<\infty$ almost surely, or $\eta(S)=\infty$ almost surely; therefore we have obtained that $\|\min \{S, 1\}\|_{1}<\infty$ if and only if $\eta(S)<\infty$ almost surely.

To prove the second statement, assume first that the conditions (a)-(c) hold. Condition (a) then implies that a.s., no Poisson point falls into the set $\left\{D_{W}=\infty\right\}$, which means we may replace $\Omega$ by a space such that $D_{W}(x)<\infty$ for all $x \in \Omega$. Let $\Omega_{>D}=\left\{x \in \Omega: D_{W}(x)>D\right\}$ and $\Omega_{\leq D}=\Omega \backslash \Omega_{>D}$. Since $\Omega_{>D}$ has finite measure by assumption (b), we have that a.s., only finitely many Poisson points fall into this set, which in particular implies that the contribution of the points $x_{i}, x_{j} \in \Omega_{>D}$ to $\eta^{2}(W)$ is a.s. finite. Next let us consider the contributions to $\eta^{2}(W)$ from pairs of points $x_{i}, x_{j}$ such that one lies in $\Omega_{>D}$ and the other one lies in $\Omega_{\leq D}$. Observing that the Poisson process in $\Omega_{>D}$ and $\Omega_{\leq D}$ are independent, and that a.s., there are only finitely many points in $\Omega_{>D}$, it will clearly be enough to show that for all $x \in \Omega_{>D}$, a.s. with respect to the Poisson process in $\Omega_{\leq D}$,

$$
\sum_{j: x_{j} \in \Omega_{\leq D}} W\left(x, x_{j}\right)<\infty .
$$


But by Lemma A.1 (1) applied to the function $S^{\prime}: \Omega_{\leq D} \rightarrow \mathbb{R}_{+}$defined by $S^{\prime}(y)=$ $W(x, y)$, the expectation of this quantity is equal to

$$
\int_{\Omega_{\leq D}} S^{\prime}(y) d \mu(y)=\int_{\Omega_{\leq D}} W(x, y) d \mu(y) .
$$

This is bounded by $D_{W}(x)$ and hence finite, which proves that the sum is a.s. finite. We are thus left with estimating $\eta^{2}\left(\left.W\right|_{\Omega_{\leq D}}\right)$. Again by Lemma A.1 (1), we have that $\mathbb{E}\left[\eta^{2}\left(\left.W\right|_{\Omega_{\leq D}}\right)\right]=\left\|\left.W\right|_{\Omega_{\leq D}}\right\|_{1}$ which is finite by assumption (c), showing that $\eta^{2}\left(\left.W\right|_{\Omega_{\leq D}}\right)$ is a.s. finite.

Conversely, let us assume that a.s., $\eta^{2}(W)<\infty$. First we will prove that this implies $\mu\left(\left\{D_{W}=\infty\right\}\right)=0$. Assume for a contradiction that this is not the case. Since $\mu$ is $\sigma$-finite, we can find a measurable set $N \subseteq \Omega$ such that $D_{W}(x)=\infty$ for all $x \in N$ and $0<\mu(N)<\infty$. Consider the contribution to $\eta^{2}(W)$ by all Poisson points $\left(x_{i}, x_{j}\right)$ such that $x_{i} \in N$ and $x_{j} \in N^{c}=\Omega \backslash N$. Since the Poisson processes on $N$ and $N^{c}$ are independent, the finiteness of $\eta^{2}(W)$ implies that for almost all $x \in N$, the sum $\sum_{j: x_{j} \in N^{c}} W\left(x, x_{j}\right)$ is a.s. finite. Applying statement (1) of the current proposition to $W(x, \cdot)$ (and recalling that $W$ is bounded by 1 ), we conclude that for almost all $x \in N, \int_{N^{c}} W(x, y) d \mu(y)<\infty$, which implies that for almost all $x \in N, \int_{N} W(x, y) d \mu(y)=D_{W}(x)-\int_{N^{c}} W(x, y) d \mu(y)=\infty$. This is a contradiction since $\mu(N)<\infty$ and $W \leq 1$.

We next prove (b) (for any value of $D$ ). Suppose for a contradiction that $\mu\left(\left\{x \in \Omega: D_{W}(x)>D\right\}\right)=\infty$. We then claim that almost surely, $\eta^{2}(W)=\infty$. After obtaining the Poisson process, color each point randomly red or blue, with equal probability, independently. We can then obtain the red and blue points equivalently by taking two independent Poisson processes, both with intensity $\mu / 2$. We claim that almost surely, the sum of $W(x, y)$ just over red-blue pairs is already $\infty$. We know that almost surely, there are an infinite number of red points $x_{i}$ with $D_{W}\left(x_{i}\right)>D$. Let $x_{n}$ be such a sequence, and given $y \in \Omega$, let $S^{\prime}(y)=\sum_{n=1}^{\infty} W\left(x_{n}, y\right)$. Then the sum of $W$ over red-blue edges is equal to $\eta\left(S^{\prime}\right)$ for the Poisson process with intensity $\mu / 2$. Therefore, it suffices to prove that $\left\|\min \left\{S^{\prime}, 1\right\}\right\|_{1, \mu / 2}=\infty$. First, note that if either $\mu\left(\left\{y \in \Omega: S^{\prime}(y)=\infty\right\}\right)>0$ or $\mu\left(\left\{y \in \Omega: S^{\prime}(y)>1\right\}\right)=\infty$, then it clearly holds. Otherwise, we have that as $D^{\prime} \rightarrow \infty, \mu\left(\left\{y \in \Omega: S^{\prime}(y)>D^{\prime}\right\}\right) \rightarrow 0$; therefore, there exists some $D^{\prime}$ (without loss of generality, we may assume $D^{\prime} \geq 1$ ) such that $\mu\left(\left\{y \in \Omega: S^{\prime}(y)>D^{\prime}\right\}\right)<D / 2$. Let $\Omega^{\prime}$ be the complement of $\left\{y \in \Omega: S^{\prime}(y)>D^{\prime}\right\}$. We then have that for each $x_{n}$,

$$
\begin{aligned}
\int_{\Omega^{\prime}} W\left(x_{n}, y\right) \frac{d \mu(y)}{2} & =\int_{\Omega} W\left(x_{n}, y\right) \frac{d \mu(y)}{2}-\int_{\Omega \backslash \Omega^{\prime}} W\left(x_{n}, y\right) \frac{d \mu(y)}{2} \\
& \geq \frac{1}{2} D_{W}\left(x_{n}\right)-\frac{1}{2} \mu\left(\Omega \backslash \Omega^{\prime}\right) \geq \frac{D}{2}-\frac{D}{4} .
\end{aligned}
$$

We also have that

$$
\begin{aligned}
\int_{\Omega^{\prime}} S^{\prime}(y) \frac{d \mu(y)}{2} & =\int_{\Omega^{\prime}} \sum_{n=1}^{\infty} W\left(x_{n}, y\right) \frac{d \mu(y)}{2} \\
& =\sum_{n=1}^{\infty} \int_{\Omega^{\prime}} W\left(x_{n}, y\right) \frac{d \mu(y)}{2} \geq \sum_{n=1}^{\infty} D / 4=\infty
\end{aligned}
$$


Therefore,

$$
\begin{aligned}
\int_{\Omega} \min \left\{S^{\prime}(y), 1\right\} \frac{d \mu(y)}{2} & \geq \int_{\Omega^{\prime}} \min \left\{S^{\prime}(y), 1\right\} \frac{d \mu(y)}{2} \\
& \geq \frac{1}{D^{\prime}} \int_{\Omega^{\prime}} \min \left\{S^{\prime}(y), D^{\prime}\right\} \frac{d \mu(y)}{2}=\frac{1}{D^{\prime}} \int_{\Omega^{\prime}} S^{\prime}(y) \frac{d \mu(y)}{2}=\infty .
\end{aligned}
$$

This contradiction completes the proof.

We are left with proving (c) (we will again prove it for any value of $D$ ). Assume the opposite, and let $\Lambda_{n} \subseteq \Lambda$ be an increasing sequence such that $\mu\left(\Lambda_{n}\right)<\infty$ and $\bigcup_{n} \Lambda_{n}=\Omega_{\leq D}$. Let $U_{n}=\left.W\right|_{\Lambda_{n}}$. Then $\left\|U_{n}\right\|_{1}<\infty,\left\|U_{n}\right\|_{1} \uparrow\left\|\left.W\right|_{\Omega_{\leq D}}\right\|_{1}=\infty$, and $\left\|D_{U_{n}}\right\|_{\infty} \leq D$, implying in particular that $\left\|U_{n} \circ U_{n}\right\|_{1}=\left\|D_{U_{n}}\right\|_{2}^{2} \leq D\left\|D_{U_{n}}\right\|_{1}=$ $D\left\|U_{n}\right\|_{1}$. Given an arbitrary constant $\lambda$, we claim that

$$
\mathbb{P}\left(\eta^{2}\left(\left.W\right|_{\Omega_{\leq D}}\right)>\lambda\right) \geq \frac{\left(\left\|U_{n}\right\|_{1}-\lambda\right)^{2}}{\left\|U_{n}\right\|_{1}^{2}+(4 D+2)\left\|U_{n}\right\|_{1}},
$$

provided $n$ is large enough to ensure that $\left\|U_{n}\right\|_{1}>\lambda$. Indeed, writing

$$
\mathbb{E}\left[\eta^{2}\left(U_{n}\right)\right]=\mathbb{E}\left[\eta^{2}\left(U_{n}\right) 1_{\eta^{2}\left(U_{n}\right) \leq \lambda}\right]+\mathbb{E}\left[\eta^{2}\left(U_{n}\right) 1_{\eta^{2}\left(U_{n}\right)>\lambda}\right],
$$

we can bound the first term by $\lambda$ and the second by $\sqrt{\mathbb{E}\left[\left(\eta^{2}\left(U_{n}\right)\right)^{2}\right] \mathbb{P}\left[\eta^{2}\left(U_{n}\right)>\lambda\right]}$, using Cauchy's inequality. We therefore obtain that

$$
\mathbb{E}\left[\eta^{2}\left(U_{n}\right)\right] \leq \lambda+\sqrt{\mathbb{E}\left[\left(\eta^{2}\left(U_{n}\right)\right)^{2}\right] \mathbb{P}\left[\eta^{2}\left(U_{n}\right)>\lambda\right]} .
$$

Rearranging, we obtain the bound

$$
\mathbb{P}\left(\eta^{2}\left(U_{n}\right)>\lambda\right) \geq \frac{\left(\mathbb{E}\left[\eta^{2}\left(U_{n}\right)\right]-\lambda\right)^{2}}{\mathbb{E}\left[\left(\eta^{2}\left(U_{n}\right)\right)^{2}\right]}=\frac{\left(\left\|U_{n}\right\|_{1}-\lambda\right)^{2}}{\left\|U_{n}\right\|_{1}^{2}+4\left\|U_{n} \circ U_{n}\right\|_{1}+\left\|U_{n}^{2}\right\|_{1}},
$$

where we used Lemma A.1 (1) and (3) in the last step. Observing that

$$
\operatorname{Pr}\left(\eta^{2}\left(\left.W\right|_{\Omega_{\leq D}}\right)>\lambda\right) \geq \operatorname{Pr}\left(\eta^{2}\left(U_{n}\right)>\lambda\right)
$$

and bounding $4\left\|U_{n} \circ U_{n}\right\|_{1}+\left\|U_{n}^{2}\right\|_{1}$ by $(4 D+2)\left\|U_{n}\right\|_{1}$, we obtain (A.1). Since the right side of (A.1) goes to 1 as $n \rightarrow \infty$, we get that with probability one, $\eta^{2}\left(\left.W\right|_{\Omega_{\leq D}}\right)>\lambda$ for all $\lambda$, which contradicts the assumption that $\eta^{2}\left(\left.W\right|_{\Omega_{\leq D}}\right)<\infty$ a.s.

Proof of Proposition 2.4. We first prove the equivalence of (A) - (E). Clearly $(B) \Rightarrow$ $(C) \Rightarrow(A)$ and $(D) \Rightarrow(E)$. It is also not hard to see that $(E) \Rightarrow(A)$. Indeed, note first that for any $D$, the condition on $S$ is equivalent to the condition that $\min \{S, D\}$ is integrable (which implies that $\mu(\{S>D\})<\infty$.) Set $\Omega^{\prime}=\left\{D_{W} \leq D\right\} \cap\{S \leq D\}$. Then (E) implies that

$$
\begin{aligned}
\left\|\left.\mathbb{W}\right|_{\Omega^{\prime}}\right\|_{1} & \leq 2 I+\left\|W_{\left\{D_{W} \leq D\right\}}\right\|_{1}+2\left\|S 1_{S \leq D}\right\|_{1} \\
& \leq 2 I+\left\|W_{\left\{D_{W} \leq D\right\}}\right\|_{1}+2\|\min \{S, D\}\|_{1}<\infty
\end{aligned}
$$

and $\mu\left(\Omega \backslash \Omega^{\prime}\right) \leq \mu\left(\left\{D_{W}>D\right\}\right)+\mu(\{S>D\})<\infty$, proving (A). So it will be enough to show $(A) \Rightarrow(B)$ and $(A) \Rightarrow(D)$.

Suppose that (A) holds, and let $\Omega^{\prime}$ be a set such that $\mu\left(\Omega \backslash \Omega^{\prime}\right)<\infty, \mathbb{W}^{\prime}=\left.\mathbb{W}\right|_{\Omega^{\prime}}$, and $\left\|\mathbb{W}^{\prime}\right\|_{1}=C<\infty$. Let $D>0$. First, assume that $D>D_{0}=\mu\left(\Omega \backslash \Omega^{\prime}\right)$. Then

$$
\left\{x \in \Omega: D_{\mathbb{W}}(x)>D\right\} \subseteq\left(\Omega \backslash \Omega^{\prime}\right) \cup\left\{x \in \Omega^{\prime}, D_{\mathbb{W}^{\prime}}(x)>D-D_{0}\right\} .
$$


Since $\left\|D_{\mathbb{W}^{\prime}}\right\|_{1} \leq\left\|\mathbb{W}^{\prime}\right\|_{1}=C$, this set has measure at most

$$
D_{0}+\frac{C}{D-D_{0}} .
$$

Now, let $\mathbb{W}^{\prime \prime}=\left.\mathbb{W}\right|_{\left\{x: D_{\mathbb{W}}(x) \leq D\right\}}$. Then

$$
\left\|\mathbb{W}^{\prime \prime}\right\|_{1} \leq\left\|\mathbb{W}^{\prime}\right\|_{1}+2 \int_{\left\{x \in \Omega \backslash \Omega^{\prime}: D_{\mathbb{W}}(x) \leq D\right\}} D_{\mathbb{W}}(x) \leq\left\|\mathbb{W}^{\prime}\right\|_{1}+2 D D_{0} .
$$

We have thus proven that (B) holds for all $D$ larger than some $D_{0}$, and more generally for any $D$ for which there exists an $\Omega^{\prime} \subseteq \Omega$ with $\mu\left(\Omega \backslash \Omega^{\prime}\right)<D$ and $\left\|\left.\mathbb{W}\right|_{\Omega^{\prime}}\right\|_{1}<\infty$.

Note that if $\left.\mathbb{W}\right|_{\left\{x: D_{\mathbb{W}}(x) \leq D\right\}}$ is integrable for $D>D_{0}$, then it must remain integrable if we decrease $D$, since that is just a restriction to a subset. Therefore, this implies that $\left.\mathbb{W}\right|_{\left\{x: D_{\mathbb{W}}(x) \leq D\right\}}$ is integrable for all $D$. Since $D_{\mathbb{W}}<\infty$ almost everywhere, we further have that $\mu\left(\left\{x \in \Omega: D_{\mathbb{W}}(x) \geq \lambda\right\}\right)$ tends to 0 as $\lambda$ tends to $\infty$ (since we at least know that it is finite for large enough $\lambda$ ). Fixing $D>0$, we can therefore take $D^{\prime}$ large enough so that $\mu\left(\left\{x \in \Omega: D_{\mathbb{W}}(x) \geq D^{\prime}\right\}\right)<D$. Taking $\Omega^{\prime}:=\Omega \backslash\left\{x \in \Omega: D_{\mathbb{W}}(x) \geq D^{\prime}\right\}$, we get a set $\Omega^{\prime}$ such that $\mu\left(\Omega \backslash \Omega^{\prime}\right)<D$ and $\left\|\left.\mathbb{W}\right|_{\Omega^{\prime}}\right\|_{1}<\infty$ proving that (B) holds for all $D>0$.

On the other hand if (A) holds for some $\Omega^{\prime}$, then $\left\|\left.W\right|_{\Omega^{\prime}}\right\|_{1}<\infty$ and $\left\|S 1_{\Omega^{\prime}}\right\|_{1}<\infty$. Proceeding exactly as above we conclude that for all $D, \mu\left(\left\{D_{W}>D\right\}\right)<\infty$ and $\left\|\left.W\right|_{\left\{D_{W} \leq D\right\}}\right\|_{1}<\infty$, as well as $\mu(\{S>D\})<\infty$ and $\left\|S 1_{\{S \leq D\}}\right\|_{1}<\infty$. Since

$$
\|\min \{S, D\}\|_{1}=D \mu(\{S>D\})+\left\|S 1_{\{S \leq D\}}\right\|_{1}<\infty,
$$

the latter condition is equivalent to $\|\min \{S, D\}\|_{1}<\infty$, as required.

We are left with proving that the local finiteness conditions in Definition 2.1 are necessary and sufficient for the almost sure finiteness of $G_{T}(\mathbb{W})$ for all $T<\infty$. It is easy to check that the local finiteness conditions are not affected if we multiply the underlying measure by $T$ and $S$ by $T$. We therefore assume that $T=1$. Let $\eta=\sum_{i} \delta_{x_{i}}$ be a Poisson process of intensity $\mu$ on $\Omega$, let $Y_{i}$ be Poisson random variable with mean $S\left(x_{i}\right)$, and let $Y_{i j}$ be Bernoulli with mean $W\left(x_{i}, x_{j}\right)$, all of them independent of each other. We will have to show that the local finiteness conditions on $\mathbb{W}$ are equivalent to the a.s. finiteness of the sums

$$
e_{S}=\sum_{i} Y_{i} \quad \text { and } \quad e_{W}=\sum_{i>j} Y_{i j} .
$$

We next use the fact that a sum of independent, non-negative random variables $\sum_{k} Z_{k}$ is a.s. finite if and only if $\sum_{i} \mathbb{E}\left[\min \left\{Z_{i}, 1\right\}\right]<\infty$. In the case of $e_{W}, Y_{i, j}$ is bounded, and therefore we immediately have that $e_{W}$ is a.s. finite if and only if $\eta^{2}(W)$ is a.s. finite. Proposition A.2 (b) then proves this case. In the case of $e_{S}$, setting $S^{\prime}=\min \{S, 1\}$, applying Proposition A.2 to $S^{\prime}$, and noting that $S^{\prime}$ is bounded, we have that $\sum_{i} S^{\prime}\left(x_{i}\right)$ is almost surely finite if and only if $\left\|S^{\prime}\right\|_{1}<\infty$. This is exactly the condition on $S$.

\section{Appendix B. SAmpling With LOOPS}

In this section, we discuss how to handle samples with loops. The sampling process is adjusted as follows. We follow the same process as for $\mathcal{G}_{T}(\mathbb{W})$ and $\mathcal{G}_{\infty}(\mathbb{W})$; however, for each vertex labeled as $(t, x)$, with probability $W(x, x)$, we add a loop to the vertex. Deleting isolated vertices as before, and then removing the feature 
labels from the vertices, we obtain a family $\left(\widetilde{\mathcal{G}}_{T}(\mathbb{W})\right)_{T \geq 0}$ of labelled graphs with loops, as well as the infinite graph $\widetilde{\mathcal{G}}_{\infty}(\mathbb{W})=\bigcup_{T>0} \widetilde{\mathcal{G}}_{T}(\mathbb{W})$.

Note that a vertex that was previously isolated may not be isolated anymore if it receives a loop, so a vertex may have been deleted from $\mathcal{G}_{T}(\mathbb{W})$ but not from $\widetilde{\mathcal{G}}_{T}(\mathbb{W})$. We add a further condition for local finiteness:

$$
\int_{\Omega} W(x, x) d \mu(x)<\infty .
$$

Note that if $\mathbb{W}$ is atomless, then the values $W(x, x)$ do not have an effect on $\mathcal{G}_{T}$ and $\mathcal{G}_{\infty}$, and the diagonal constitutes a zero measure set in $\Omega \times \Omega$.

As stated, Theorem 2.5 is false for sampling with loops. Since the diagonal may be a zero measure set, almost everywhere equal pullbacks do not imply having the same looped samples. We could further add the condition that $W(x, x)$ is equal to the pullback almost everywhere, but the theorem would still be false. This is demonstrated by the following example. Let $\boldsymbol{\Omega}_{1}=\boldsymbol{\Omega}_{2}=[0,1]$. Take $W_{1}$ to be constant $1 / 2$ on $[0,1] \times[0,1]$, and let $W_{2}$ be constant $1 / 2$ off the diagonal, 0 if $x<1 / 2$, 1 otherwise. Let $\mathbb{W}_{i}=\left(W_{i}, 0,0, \boldsymbol{\Omega}_{i}\right)$. Then we claim that $\widetilde{\mathcal{G}}_{T}\left(\mathbb{W}_{1}\right)$ and $\widetilde{\mathcal{G}}_{T}\left(\mathbb{W}_{2}\right)$ have the same distribution. Indeed, both are equivalent to taking Poisson $(T)$ vertices, adding a loop to each vertex with probability $1 / 2$, independently, and also taking an edge between each pair of vertices with probability $1 / 2$, independently over different pairs.

It turns out that in general, allowing diagonal values strictly between 0 and 1 is not necessary, because we could extend the feature space to determine whether each vertex has loops. For graphexes where the diagonal is 0 or 1 , we can then conclude an analogous theorem from Theorem 2.5.

We first show the following:

Proposition B.1. For any graphex $\mathbb{W}=(W, S, I, \boldsymbol{\Omega})$, there exists a graphex $\widetilde{\mathbb{W}}=$ $(\widetilde{W}, \widetilde{S}, \widetilde{I}, \widetilde{\boldsymbol{\Omega}})$ on an atomless space $\widetilde{\boldsymbol{\Omega}}$ such that on the diagonal, $\widetilde{W}$ is $\{0,1\}$ valued and such that $\widetilde{\mathcal{G}}_{\infty}(\widetilde{\mathbb{W}})$ and $\widetilde{\mathcal{G}}_{T}(\widetilde{\mathbb{W}})$ are equivalent to $\widetilde{\mathcal{G}}_{\infty}(\mathbb{W})$ and $\widetilde{\mathcal{G}}_{T}(\mathbb{W})$, respectively.

Proof. Let $\widetilde{\boldsymbol{\Omega}}=\boldsymbol{\Omega} \times[0,1]$, and let $\pi_{1}, \pi_{2}$ be the projection maps. Note that we can obtain a Poisson process on $\widetilde{\boldsymbol{\Omega}} \times \mathbb{R}_{+}$by taking a Poisson process on $\boldsymbol{\Omega} \times \mathbb{R}$, and independently labeling each point with a uniform random real number from $[0,1]$, which becomes the second coordinate. Clearly $\widetilde{\boldsymbol{\Omega}}$ is atomless, so the diagonal values only affect the generation of the loops. Define $\widetilde{I}=I, \widetilde{S}=S \circ \pi_{1}, \widetilde{W}(x, y)=$ $W\left(\pi_{1}(x), \pi_{1}(y)\right)$ if $x \neq y$, and

$$
\widetilde{W}(x, x)= \begin{cases}1, & \text { if } \pi_{2}(x) \leq W\left(\pi_{1}(x), \pi_{1}(x)\right) \\ 0, & \text { otherwise }\end{cases}
$$

Then the sampling of edges between vertices is not affected by the second coordinate of a vertex. Note that the probability that there exist two vertices corresponding to the same point in $\widetilde{\boldsymbol{\Omega}}$ is zero, since $\widetilde{\boldsymbol{\Omega}}$ is atomless. For the loops, since we can obtain the vertices by first taking the Poisson process on $\Omega \times \mathbb{R}$ and then randomly labeling each vertex with a $[0,1]$ real number, we can see that for a point $y \in \Omega$, if it ends up as a point, there is a $W(y, y)$ probability that the point $x$ corresponding to it has $\widetilde{W}(x, x)=1$, and $1-W(y, y)$ that $\widetilde{W}(x, x)=0$, and this is independent over different points. Therefore, the distribution of loops is the same. 
Using this proposition, sampling loops according to the diagonal is equivalent to the following theory. The objects are graphexes with special subsets $\mathbb{W}=$ $(W, S, I, \boldsymbol{\Omega}, A)$ where $W, S, I$, and $\boldsymbol{\Omega}$ are as before, and the special set $A \subseteq \Omega$ is a measurable subset with finite measure. We sample $\widetilde{\mathcal{G}}_{\infty}(\mathbb{W})$ in the same way as $\mathcal{G}_{\infty}(\mathbb{W})$, except that we add a loop to each vertex with a feature label in $A$. We then take the non-isolated vertices with time label at most $T$ for $\widetilde{\mathcal{G}}_{T}(\mathbb{W})$. We can extend the definition of measure-preserving map by requiring that points in the special set be mapped to points in the special set, and points not in the special set be mapped to points not in the special set. We also define dsupp as earlier, except it contain all points in $A$ (even if otherwise they would not be included).

Theorem B.2. Let $\mathbb{W}_{1}$ and $\mathbb{W}_{2}$ be graphexes with special subsets as above. Then $\widetilde{\mathcal{G}}_{T}\left(\mathbb{W}_{1}\right)$ and $\widetilde{\mathcal{G}}_{T}\left(\mathbb{W}_{2}\right)$ have the same distribution for all $T \in \mathbb{R}_{+}$if and only if there exists a third graphex with special subset $\mathbb{W}$ such that $\mathbb{W}_{1}$ and $\mathbb{W}_{2}$ are pullbacks of $\mathbb{W}$.

Proof. It is clearly enough to prove the only if direction. Suppose therefore that $\mathbb{W}_{1}$ and $\mathbb{W}_{2}$ have the same distribution. Then for any $0<c<1, c \mathbb{W}_{1}$ and $c \mathbb{W}_{2}$ have the same distributions (i.e., $W, S, I$ are all multiplied by $c$, and the special set stays the same). Then let $\widetilde{\mathbb{W}}_{i}$ be obtained by taking $\mathbb{W}_{i} / 2$, adding a set $B_{i}$ of measure 1 to $\Omega_{i}$, and extending $W_{i}$ to be 1 on $B_{i} \times B_{i}, 1$ between $B_{i}$ and $A_{i}$, and 0 between $B_{i}$ and $\Omega_{i} \backslash A_{i}$. Then we can obtain $G_{T}\left(\widetilde{\mathbb{W}}_{i}\right)$ from $\widetilde{G}_{T}\left(\mathbb{W}_{i}\right)$ by the following process. We first keep each edge that is not a loop with probability $1 / 2$, and delete it otherwise, independently. We keep all the loops. Then we take Poisson $(T)$ new vertices, put an edge between every pair, and put an edge between each new vertex and each vertex that had a loop (and delete loops). It is clear that in this way, the distributions $G_{T}\left(\widetilde{\mathbb{W}}_{1}\right)$ and $G_{T}\left(\widetilde{\mathbb{W}}_{2}\right)$ are the same for every $T$. Therefore, there exists a graphex $\widetilde{\mathbb{W}}=(\widetilde{W}, \widetilde{S}, \widetilde{I}, \widetilde{\Omega})$ such that $\widetilde{\mathbb{W}}_{1}$ and $\widetilde{\mathbb{W}}_{2}$ are both pullbacks of $\widetilde{\mathbb{W}}$. It is clear that $\widetilde{\mathbb{W}}$ must have a set of measure 1 , call it $B$, which has $\widetilde{W}(x, y)=1$ if $x, y \in B$, and $\widetilde{W}(x, y)$ is either 0 or 1 if $x \in B, y \notin B$, and only depends on $y$, and $\widetilde{W}(x, y) \leq 1 / 2$ if $x, y \notin B$, and $B$ must pullback to exactly $B_{1}$ and $B_{2}$. If we let $A$ be the set of points $x$ with $\widetilde{W}(x, y)=1$ for any and all $y \in B$, then $A$ must pullback to $A_{1}$ and $A_{2}$. If we therefore let $\mathbb{W}$ have underlying set $\widetilde{\Omega} \backslash B$, and be equal to $2 \widetilde{\mathbb{W}}$ restricted to this set, and special set $A$, then $\mathbb{W}$ pulls back to both $\mathbb{W}_{1}$ and $\mathbb{W}_{2}$.

\section{REFERENCES}

[1] P. Billingsley, Convergence of Probability Measures, Wiley, New York, 1968.

[2] C. Borgs, J. T. Chayes, H. Cohn, and N. Holden, in preparation, 2018.

[3] C. Borgs, J. T. Chayes, H. Cohn, and N. Holden, Sparse exchangeable graphs and their limits via graphon processes, arXiv:1601.07134, to appear in Journal of Machine Learning Research.

[4] C. Borgs, J. T. Chayes, H. Cohn, and V. Veitch, Sampling perspectives on sparse exchangeable graphs, arXiv:1708.03237.

[5] C. Borgs, J. T. Chayes, H. Cohn, and Y. Zhao, An $L^{p}$ theory of sparse graph convergence I: limits, sparse random graph models, and power law distributions, arXiv:1401.2906, to appear in Transactions of the American Mathematical Society.

[6] C. Borgs, J. T. Chayes, S. Dhara, and S. Sen, Limits of sparse configuration models and beyond: graphexes and multi-graphexes, in preparation, 2018.

[7] C. Borgs, J. T. Chayes, L. Lovász, V. Sós, and K. Vesztergombi, Counting graph homomorphisms, Topics in Discrete Mathematics (M. Klazar, J. Kratochvíl, M. Loebl, J. Matoušek, R. Thomas, and P. Valtr, eds.), Springer, 2006, pp. 315-371. 
[8] C. Borgs, J. T. Chayes, L. Lovász, V. Sós, and K. Vesztergombi, Convergent graph sequences I: subgraph frequencies, metric properties, and testing, Advances in Math. 219 (2008), 1801-1851.

[9] C. Borgs, J. T. Chayes, L. Lovász, V. Sós, and K. Vesztergombi, Convergent graph sequences II: multiway cuts and statistical physics, Ann. of Math. 176 (2012), 151-219.

[10] C. Borgs, J. Chayes, and L. Lovász, Moments of two-variable functions and the uniqueness of graph limits, Geom. Funct. Anal. 19 (2010), 1597-1619.

[11] F. Caron and E. B. Fox, Sparse graphs using exchangeable random measures, J. R. Stat. Soc. Ser. B. Stat. Methodol. 79 (2017), 1295-1366.

[12] D. L. Cohn, Measure Theory, second edition. Birkhäuser Advanced Texts: Basler Lehrbücher. Birkhäuser/Springer, New York, 2013.

[13] D. J. Daley and D. Vere-Jones, An Introduction to the Theory of Point Processes: Volume I: Elementary Theory and Methods, second edition, Springer, 2003.

[14] A. Frieze and R. Kannan, Quick approximation to matrices and applications, Combinatorica 19 (1999), 175-220.

[15] W. Hoeffding, Probability inequalities for sums of bounded random variables, Journal of the American Statistical Association 58 (1963), 13-30.

[16] S. Janson, Graphons, cut norm and distance, couplings and rearrangements, New York Journal of Mathematics. NYJM Monographs, vol. 4, State University of New York, University at Albany, Albany, NY, 2013.

[17] S. Janson, Graphons and cut metric on sigma-finite measure spaces, arXiv:1608.01833, 2016.

[18] S. Janson, On convergence for graphexes, arXiv:1702.06389, 2017.

[19] O. Kallenberg, Probabilistic Symmetries and Invariance Principles, Springer, 2005.

[20] D. Kunszenti-Kovács, L. Lovász, and B. Szegedy, Multigraph limits, unbounded kernels, and Banach space decorated graphs, arXiv:1406.7846, 2014.

[21] L. Lovász and B. Szegedy, Limits of dense graph sequences, J. Combin. Theory Ser. B 96 (2006), 933-957.

[22] L. Lovász and B. Szegedy, Szemerédi's lemma for the analyst, Geom. Funct. Anal. 17 (2007), $252-270$.

[23] L. Lovász and B. Szegedy, Regularity partitions and the topology of graphons, in An Irregular Mind, Bolyai Soc. Math. Stud., vol. 21, János Bolyai Math. Soc., Budapest, 2010, pp. 415-446.

[24] W. Rudin, Real and Complex Analysis, third edition, McGraw-Hill Book Co., New York, 1987.

[25] V. Veitch and D. M. Roy, Sampling and estimation for (sparse) exchangeable graphs, arXiv:1611.00843, 2016.

[26] V. Veitch and D. M. Roy, The class of random graphs arising from exchangeable random measures, arXiv:1512.03099, 2015.

Microsoft Research, One Memorial Drive, Cambridge, MA 02142

E-mail address: borgs@microsoft.com

Microsoft Research, One Memorial Drive, Cambridge, MA 02142

E-mail address: jchayes@icrosoft.com

Microsoft Research, One Memorial Drive, Cambridge, MA 02142

E-mail address: cohn@microsoft.com

Mathematics Department, UCla, Los Angeles, CA 90095

E-mail address: lmlovasz@math.ucla.edu 